\title{
A função período para uma classe de sistemas hamiltonianos
}

\author{
Emivan Ferreira da Silva
}

DISSERTAÇÃO APRESENTADA

$\mathrm{AO}$

INSTITUTO DE MATEMÁTICA E ESTATÍSTICA

DA

UNIVERSIDADE DE SÃO PAULO

PARA

OBTENÇÃO DO GRAU DE MESTRE

EM

MATEMÁTICA APLICADA

Área de concentração: Matemática Aplicada

Orientadora: Profa. Dra. Sônia Regina Leite Garcia

São Paulo, Novembro de 2002 


\section{A função período para uma classe de sistemas hamiltonianos}

Este exemplar corresponde à redação final da dissertação devidamente corrigida e defendida por Emivan Ferreira da Silva e aprovada pela comissão julgadora.

São Paulo, 26 de novembro de 2002.

Banca examinadora:

- Profa. Dra. Sônia Regina Leite Garcia (orientadora) - IME-USP

- Profa. Dra. Roseli Fernandez - IME-USP

- Prof. Dr. Clodoaldo Glotta Ragazzo - IME-USP 
À minha esposa, Adriana Souza Resende, aos meus filhos: Melissa, Ivan Gabriel e Yasmin. e aos meus pais: José F. Lustosa e Divina F. da Silva. 


\section{Agradecimentos}

Enfim os agradecimentos, terei muito prazer e orgulho em digitar cada nome a seguir pois revelará minha gratidão a todos eles.

- A Deus, por estar presente em todos os momentos de minha vida.

- À minha querida esposa Adriana Souza Resende por sacrificar-se continuamente em favor da minha dedicação ao estudo e ao trabalho que resultou nessa dissertação e por ser compreensiva nos momentos de angústia e mal humor causados pela insegurança e pelos problemas naturais que se pode esperar de um trabalho como este.

- Aos meus filhos, Melissa R. Ferreira e Ivan Gabriel R. Ferreira pelo carinho apesar da minha constante ausência.

- À Prof. Dra Sônia Regina Leite Garcia pela paciência e compreensão com que tem me acompanhado desde 1997 neste instituto, com quem muito aprendi durante estes anos de convivência. Obrigado pelo incentivo e apoio.

- À minha querida irmã Deuzimar F. da Silva, que no meu primeiro ano de mestrado contribuiu de forma decisiva ajudando nos afazeres domésticos enquanto eu me dedicava aos estudos.

- Aos meus pais José F. Lustosa e Divina F. da Silva, que apesar da distância sempre acreditaram e confiaram em mim.

- A todos os colegas do IME-USP que de alguma forma contribuíram com esse meu sonho, em especial os amigos Antônio Ronaldo G. Garcia, Mํㅡ do Carmo P. de Souza, $M^{\underline{a}}$ Cecília A. L. Barto, Olga H. Saito, Rudimar L. Nos, Gisele A. A. Sanchez, Tatiane M. Pereira, Antônio Noel, Célia M. C. Lopes, Jocirei D. Ferreira, David A. Z. Villanueva, German C. Losada e Santos A. E. Remigio.

- Aos meus colegas da EE João XXIII pelo carinho e atenção com que sempre se dispuseram a ajudar em pequenos detalhes que se tornaram grandes na construção desse trabalho, em especial cito as professoras Elza Yuri, Ana $M^{\underline{a}}$ Valença, Rosângela B. Garcia (Minha conselheira), as coordenadoras Nancy A. Rocha e Lúcia Feio, a vice diretora Fátima Massay. 
- Aos professores Roseli Fernandez, Luiz A. Oliveira, Helena M. A. de Castro e Francisco Rui pelo apoio.

- À banca examinadora pelas correções e sugestões apresentadas.

A todos aqueles que com um gesto ou palavra contribuíram para este trabalho e cujos nomes não aparecem agradeço de igual modo e perdoem pelo meu esquecimento.

Emivan F. Da Silva 


\section{Resumo}

Este trabalho é uma generalização dos resultados do artigo Period Function for a Class of Hamiltonian Systems [CGM00], que estuda a função período para a classe de sistemas Hamiltonianos analíticos $\dot{x}=-H_{y}, \dot{y}=H_{x}$ com hamiltoniana natural $H(x, y)=$ $F(x)+G(y)$ onde $F$ é a energia cinética e $G$ a energia potencial, e a origem é um centro não degenerado. Mais concretamente, se $T(h)$ denota o período da órbita periódica contida na curva de nível $H(x, y)=h$, em [CGM00] é resolvido o problema inverso de caracterizar todos os sistemas com uma dada função $T$ analítica em zero. Generalizamos tal resultado para centros degenerados, caracterizando todos os sistemas hamiltonianos naturais analíticos em que $G$ pode ter mínimo degenerado em zero, com uma dada função $T$ da forma especial

$$
T(h)=\frac{1}{\sqrt[2 N]{h^{N-1}}}\left[T_{1}(h)+\sqrt[2 N]{h^{2}} T_{2}(h)+\sqrt[2 N]{h^{4}} T_{3}(h)+\cdots+\sqrt[2 N]{h^{2 N-2}} T_{N}(h)\right]
$$

com $T_{1}, T_{2}, \cdots, T_{N}$ analíticas em zero.

Apresentamos também um estudo sobre hamiltonianas analíticas não clássicas com $F$ da forma $F(x)=a x^{4}+O\left(x^{5}\right)$.

\section{Abstract}

This work generalizes the results of the paper Period Function for a Class of Hamiltonian Systems [CGM00], which studies the period function for the class of analytic Hamiltonian systems $\dot{x}=-H_{y}, \dot{y}=H_{x}$ with natural Hamiltonian function $H(x, y)=$ $F(x)+G(y)$ where $F$ is the kinetic energy and $G$ is the potential energy, with the origin being not a degenerate center. More specifically, if $T(h)$ denotes the period function of the periodic orbit contained in the level curve $H(x, y)=h$, in [CGM00] is solved the inverse problem of characterizing all the systems with one given analytic function $T$. We generalize this result for degenerate centers, characterizing all the analytic natural Hamiltonian systems where $G$ can have degenerate minimum at the origin, with $T$ given by

$$
T(h)=\frac{1}{\sqrt[2 N]{h^{N-1}}}\left[T_{1}(h)+\sqrt[2 N]{h^{2}} T_{2}(h)+\sqrt[2 N]{h^{4}} T_{3}(h)+\cdots+\sqrt[2 N]{h^{2 N-2}} T_{N}(h)\right]
$$

where $T_{1}, T_{2}, \cdots, T_{N}$ are analitic functions at the origin.

We also present a study on non classical analytic Hamiltonian functions with $F$ in the form $F(x)=a x^{4}+O\left(x^{5}\right)$. 


\section{Índice}

$\begin{array}{ll}\text { Lista de Figuras } & 1\end{array}$

$\begin{array}{ll}\text { Introdução } & 3\end{array}$

1 A função período para um sistema particular. $\quad 7$

1.1 Introdução . . . . . . . . . . . . . . . . . . . . 7

1.2 Resultados Preliminares . . . . . . . . . . . . . . . . . 10

1.3 Principais Resultados . . . . . . . . . . . . . . . . . . . 26

1.4 Sistemas Isócronos . . . . . . . . . . . . . . . . . . . 36

1.5 Monotonicidade da função período . . . . . . . . . . . . . . 38

2 Generalização dos resultados anteriores. $\quad 43$

2.1 A função período em dois exemplos ilustrativos. . . . . . . . . . . . . . . . 44

2.2 Generalização do Capítulo $1 \ldots \ldots$. . . . . . . . . . . . . 48

2.2.1 Resultados preliminares . . . . . . . . . . . . . . . 48

2.2.2 Resultado principal . . . . . . . . . . . . . . . . 61

2.2.3 Sistemas isócronos e comportamento da função período . . . . . . . 64

3 Uma generalização para sistemas hamiltonianos não clássicos. $\quad 69$

3.1 O caso em que $G$ tem mínimo não degenerado . . . . . . . . . . . . . 69

3.2 O caso em que $G$ tem mínimo degenerado . . . . . . . . . . . . . 76

A Apêndice $\quad 93$

$\begin{array}{lr}\text { Bibliografia } & 109\end{array}$ 


\section{Lista de Figuras}

1.1 Curva de nível. . . . . . . . . . . . . . . . . . . 8

1.2 Projeção da órbita periódica sobre o eixo $x \ldots \ldots \ldots \ldots \ldots$

1.3 Gráfico de $\mathrm{F} \ldots \ldots \ldots \ldots \ldots \ldots \ldots \ldots$

1.4 Órbita do sistema (1.2) contida na curva de nível $H(x, y)=h \quad \ldots \ldots \ldots$ 


\section{Introdução}

Este trabalho foi desenvolvido a partir do artigo Period Function for a Class of Hamiltonian Systems dos autores Anna Cima, Armengol Gasull e Francesc Mañosas [CGM00], que estudaram a classe de sistemas hamiltonianos da forma

$$
\left\{\begin{array}{l}
\dot{x}=-H_{y} \\
\dot{y}=H_{x}
\end{array}\right.
$$

onde $H(x, y)=F(x)+G(y)$ com $\mathrm{F}$ e $\mathrm{G}$ analíticas em zero, ambas com mínimo não degenerado em 0 e com $F(0)=G(0)=0$. Nestas condições, a origem é um centro.

O problema tratado em [CGM00] é o de caracterizar todas os sistemas da forma (1) que têm uma dada função período analítica $T$, ou seja, dada uma função período $T$ analítica em zero e uma função $F$ analítica em zero, com mínimo não degenerado, desejase encontrar ou caracterizar as funções $G$ analíticas em zero tais que o sistema (1) com $H(x, y)=F(x)+G(y)$ tenha um centro com o período da órbita $\gamma_{h}$ de energia $h$ sendo $T(h)$.

Apresentaremos no capítulo 1 todos os resultados desse artigo, procurando fazer de forma completa e objetiva as demonstrações. Alguns desses resultados serão enunciados e provados nesse capítulo numa forma mais forte do que aquela apresentada no artigo. Na seção 1.1, faremos uma pequena introdução ao capítulo e definiremos algumas funções e seus respectivos domínios para que possamos trabalhar de maneira clara nas próximas seções. Na seção 1.2 serão apresentados os resultados preliminares de [CGM00]: a criação de uma associação natural entre as energias cinéticas $F$ [respectivamente, energias potenciais $G$ ] consideradas e os pares de funções $(l, \sigma)$, onde $l(h)$ é o diâmetro da órbita $\gamma_{h}$ de (1) na direção do eixo dos $x$ [respectivamente, eixo dos $y$ ] e $\sigma$ é uma involução estrita 
analítica; a caracterização das energias potenciais $G$ para as quais as órbitas $\gamma_{h}$ do sistema (1) têm diâmetros $l(h)$ dados; a demonstração de que a função período $T$ do sistema (1) é dada pela convolução $l_{F}^{\prime} * l_{G}^{\prime}$ onde $l_{F}(h)$ e $l_{G}(h)$ são respectivamente os diâmetros da órbita $\gamma_{h}$ de (1) na direção do eixo dos $x$ e na direção do eixo dos $y$; a prova da existência e unicidade de solução, numa classe especial de funções, para a equação integral $T=l_{F} * \eta$ e para a equação integral $T=\eta * \eta$, onde $T$ e $l_{F}$ são dadas. Na seção 1.3 serão apresentados os principais resultados do artigo: a caracterização das hamiltonianas analíticas $H$ com mínimo não degenerado na origem, para as quais o sistema (1) tem uma dada função período $T$. Tal caracterização é feita tanto no caso em que $H(x, y)=F(x)+G(y)$ quanto no caso em que $H(x, y)=F(x)+F(y)$ (Teoremas 1.3.1, 1.3.2). Na seção 1.4 serão apresentadas algumas conseqüências destes resultados trabalhando com sistemas da forma (1) em que $T$ é uma função constante, e na seção 1.5 será estudada a monotonicidade de $T$.

No capítulo 2 apresentaremos nossa principal contribuição: uma generalização dos resultados do artigo [CGM00]. Esse capítulo apresentará a mesma estrutura do capítulo 1, manteremos as hipóteses sobre a energia cinética $F$, porém a energia potencial $G$, analítica e com mínimo na origem, passará a ser da forma

$$
G(y)=b y^{2 N}+O\left(y^{2 N+1}\right), \text { com } b>0, \text { onde } N \in \mathbb{N}^{*} .
$$

Neste caso, $T$ deixa de ser analítica, exceto no caso em que $N=1$, mas mantém uma forma especial, conforme mostraremos no lema 2.2.6 da seção 2.2. Outros resultados, análogos aos resultados preliminares do capítulo 1, também serão obtidos na seção 2.2 do capítulo 2, mas, é claro, com $T$ não mais analítica e sim, nessa classe determinada no lema 2.2.6 do capítulo 2. Finalmente, ainda na seção 2.2 do capítulo 2, apresentaremos nosso principal resultado: a caracterização de todos os sistemas da forma (1) com $F$ e $G$ tendo mínimo local estrito na origem e $F^{\prime \prime}(0) \neq 0$, que têm uma dada função período na classe determinada no lema 2.2.6.

Embora tenhamos obtido diversos resultados analogos aos do capítulo 1, é evidente que o fato de estarmos agora permitindo que $G$ tenha mínimo degenerado na origem fará com que alguns resultados não sejam possíveis, como é o caso dos sistemas isócronos tratados na seção 1.4, que só valem quando $N=1$. No entanto, apresentaremos um resultado que caracteriza o caso em que $\sqrt[2 N]{h^{N-1}} T, N \in \mathbb{N}^{*}$ é constante.

No capítulo 3 provaremos resultados análogos aos do capítulo 1, porém para sistemas hamiltonianos não clássicos, isto é, $F$ será de ordem 4 na origem $\left(F(x)=a x^{4}+O\left(x^{5}\right)\right.$, 
$a>0$ ) ao invés de ser de ordem 2. Inicialmente consideraremos $G(y)=b y^{2}+O\left(y^{3}\right), b>0$, e depois trataremos do caso em que $G(y)=b y^{4}+O\left(y^{5}\right), b>0$. Também nestes casos $T$ estará numa classe especial de funções e nossos resultados levarão isso em conta. Nossos principais resultados nesse capítulo são a caracterização dos sistemas considerados com uma dada função período. Neste contexto, estudaremos também o caso em que $F$ e $G$ são iguais, e obteremos uma extensão do resultado sobre isocronismo do capítulo 1, isto é, um resultado sobre o caso em que $\sqrt[2 N]{h^{N-1}} T, N \in \mathbb{N}^{*}$ é constante com $N=2$. A generalização para $F$ da forma $F(x)=a x^{2 M}+O\left(x^{2 M+1}\right)$ e $G$ é da forma $G(y)=a y^{2 N}+O\left(y^{2 N+1}\right)$ parecenos poder ser feita sem problemas, sendo apenas tediosa devido aos cálculos envolvidos.

Para facilitar a leitura e compreensão de algumas demonstrações, colocamos no Apêndice A alguns resultados usados, principalmente algumas propriedades das funções $\Gamma$ e $\beta$. 


\section{A função período para um sistema particular.}

\subsection{Introdução}

Vamos, neste capítulo, apresentar o artigo Period Function for a Class of Hamiltonian Systems dos autores Anna Cima, Armengol Gasull e Francesc Mañosas [CGM00], que nos motivou a escrever os capítulos seguintes. Neste capítulo, restringirnos-emos a apresentar o referido artigo, acrescentando alguns detalhes que auxiliam a compreensão dos resultados ou que nos serão úteis nos capítulos seguintes.

O objetivo em [CGM00] é estudar a função período para a classe de sistemas hamiltonianos da forma

$$
\left\{\begin{array}{l}
\dot{x}=-H_{y} \\
\dot{y}=H_{x}
\end{array}\right.
$$

onde $H(x, y)=F(x)+G(y)$, com $F$ e $G$ analíticas em zero, ambas com mínimo isolado não degenerado em 0 , e $F(0)=G(0)=0$.

Sejam $\Omega=\Omega_{F}$ e $\widetilde{\Omega}=\Omega_{G}$ os domínios de analiticidade de $F$ e $G$ respectivamente. Então existem $\bar{x}_{-}<0<\bar{x}_{+}$e $\bar{y}_{-}<0<\bar{y}_{+}$tais que

(i) $\left[\bar{x}_{-}, \bar{x}_{+}\right] \subset \Omega$

(ii) $\left[\bar{y}_{-}, \bar{y}_{+}\right] \subset \widetilde{\Omega}$

(iii) $F$ é estritamente decrescente em $\left[\bar{x}_{-}, 0\right)$ e estritamente crescente em $\left(0, \bar{x}_{+}\right]$, e mais, $F^{\prime}(x) \neq 0$ para $x$ nesses intervalos.

(iv) $G$ é estritamente decrescente em $\left[\bar{y}_{-}, 0\right)$ e estritamente crescente em $\left(0, \bar{y}_{+}\right]$, e mais, 
$G^{\prime}(y) \neq 0$ para $y$ nesses intervalos.

(v) Existe $\bar{h}>0$ tal que: $\bar{h}=F\left(\bar{x}_{-}\right)=F\left(\bar{x}_{+}\right)=G\left(\bar{y}_{-}\right)=G\left(\bar{y}_{+}\right)$.

Claramente, a órbita $\gamma_{\bar{h}}$ de energia $\bar{h}$ faz parte do centro em volta da origem. Mais que isso, o sistema 1.1 pode ser reescrito como

$$
\left\{\begin{array}{l}
\dot{x}=-g(y) \\
\dot{y}=f(x)
\end{array}\right.
$$

onde $f(x)=F^{\prime}(x)$ e $g(y)=G^{\prime}(y)$, tem um centro em $(0,0)$ e suas órbitas que passam em pontos do "disco" $D_{\bar{h}}$ limitado pela órbita $\gamma_{\bar{h}}$ fazem parte desse centro.

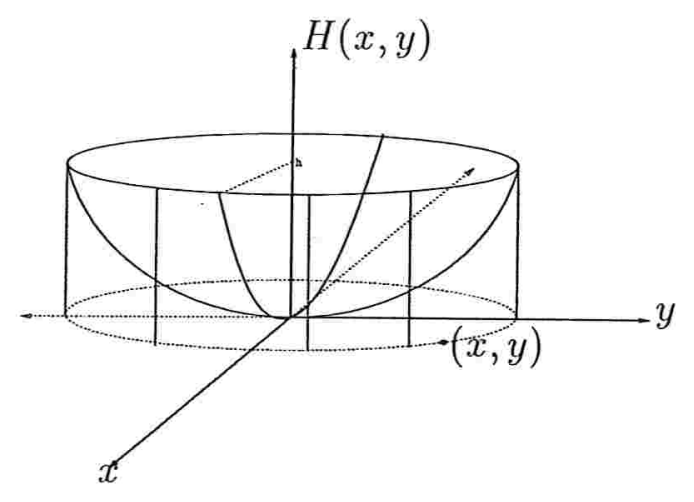

Figura 1.1: Curva de nível.

A meta principal em [CGM00] é estudar o chamado problema inverso para a função período $T$. O problema é caracterizar todos os sistemas hamiltonianos da forma (1.2) que têm uma dada função período $T$.

Vamos introduzir algumas notações que serão usadas em todo o texto.

Seja $\gamma_{h}=\left(x_{h}, y_{h}\right)$ a órbita periódica contida em $D_{\bar{h}}$ correspondendo à curva de nível $H(x, y)=h, h \in(0, \bar{h})$. Esta órbita cruza o eixo $x$ (respectivamente o eixo $y$ ) nos pontos determinados por

$$
\begin{gathered}
H\left(x_{h}, 0\right)=F\left(x_{h}\right)+G(0)=F\left(x_{h}\right)=h \\
\left(\operatorname{resp} . H\left(0, y_{h}\right)=F(0)+G\left(y_{h}\right)=G\left(y_{h}\right)=h\right) .
\end{gathered}
$$

Já que $F$ tem um único ponto crítico em $\left[x_{-}, x_{+}\right]$, que é a origem, e $F\left(\bar{x}_{+}\right)=F\left(\bar{x}_{-}\right)=\bar{h}$, então para $h \in(0, \bar{h})$ a equação $F\left(x_{h}\right)=h$ tem duas soluções em $\left(x_{-}, x_{+}\right)$, a saber, uma solução $x_{h-} \in\left(\bar{x}_{-}, 0\right)$ e outra solução $x_{h+} \in\left(0, \bar{x}_{+}\right)$. 
Note que $F_{+}=\left.F\right|_{\left(0, \bar{x}_{+}\right)}:\left(0, \bar{x}_{+}\right) \rightarrow(0, \bar{h})$ e $F_{-}=\left.F\right|_{\left(\bar{x}_{-}, 0\right)}:\left(\bar{x}_{-}, 0\right) \rightarrow(0, \bar{h})$ são inversíveis e $x_{h-}=F_{-}^{-1}(h)$ e $x_{h+}=F_{+}^{-1}(h)$.

Analogamente, $G_{+}=\left.G\right|_{\left(0, \bar{y}_{+}\right)}:\left(0, \bar{y}_{+}\right) \rightarrow(0, \bar{h})$ e $G_{-}=\left.G\right|_{\left(\bar{y}_{-}, 0\right)}:\left(\bar{y}_{-}, 0\right) \rightarrow(0, \bar{h})$ são inversíveis e a equação $G\left(y_{h}\right)=h$ tem exatamente duas soluções em $\left(y_{-}, y_{+}\right)$quando $h \in(0, \bar{h})$, que são $y_{h_{-}}=G_{-}^{-1}(h)$ e $y_{h+}=G_{+}^{-1}(h)$.

Para cada $h \in(0, \bar{h})$, definimos duas funções

$$
\left\{\begin{array}{l}
l_{F}(h)=F_{+}^{-1}(h)-F_{-}^{-1}(h) \\
l_{G}(h)=G_{+}^{-1}(h)-G_{-}^{-1}(h)
\end{array}\right.
$$

que claramente fornecem os comprimentos das projeções, nos eixos $x$ e $y$ respectivamente, da órbita periódica $\gamma_{h}$ (veja fig. 1.2). Provaremos adiante que

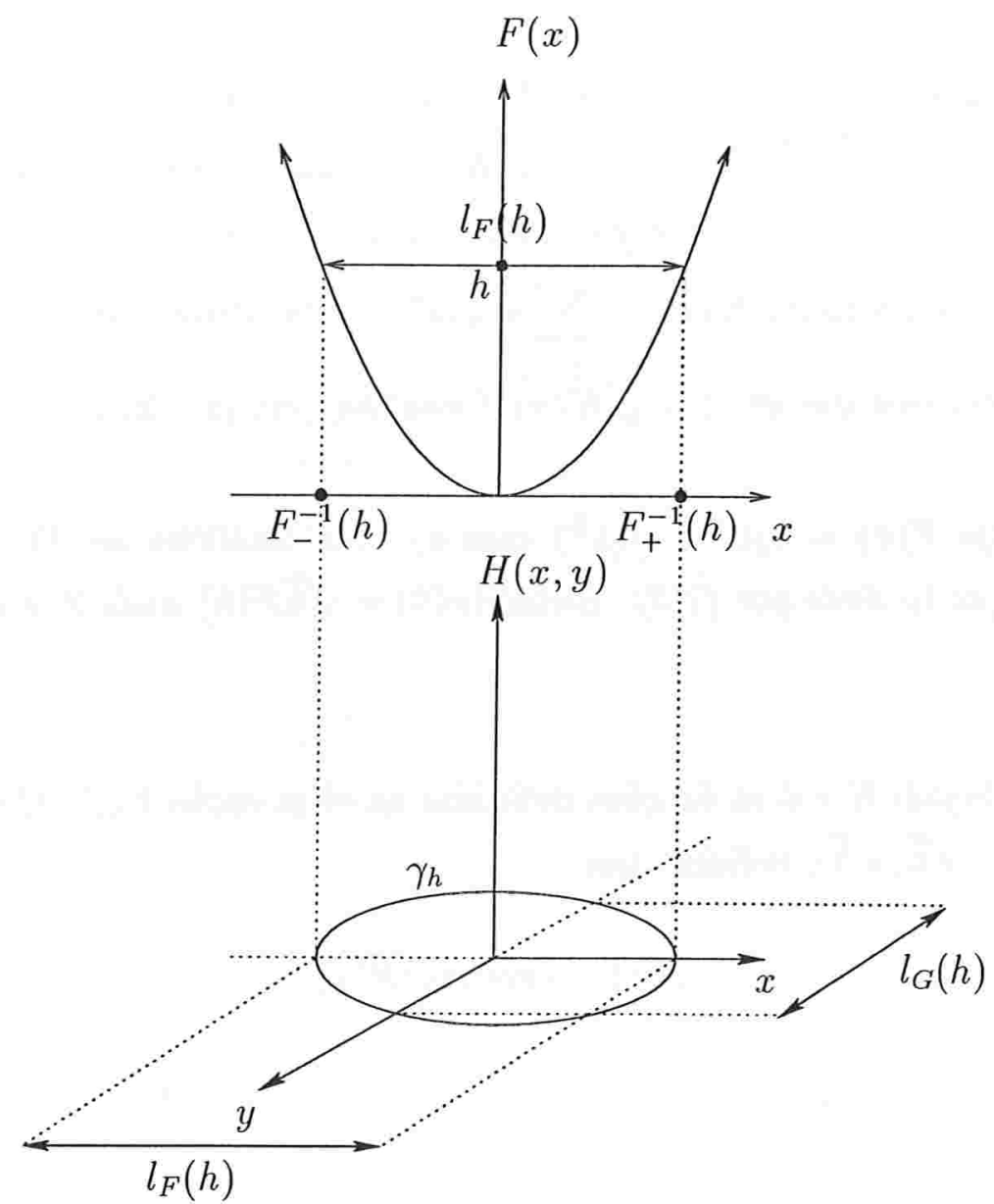

Figura 1.2: Projeção da órbita periódica sobre o eixo $x$ 


$$
\left\{\begin{array}{l}
l_{F}(h)=\sqrt{h} \mathcal{F}(h) \\
l_{G}(h)=\sqrt{h} \mathcal{G}(h)
\end{array}\right.
$$

onde $\mathcal{F}$ e $\mathcal{G}$ são funções analíticas em zero $\operatorname{com} \mathcal{F}(0)>0$ e $\mathcal{G}(0)>0$.

\subsection{Resultados Preliminares}

Vamos, nesta seção, enunciar e demonstrar alguns resultados preliminares que serão usados na demonstração dos principais resultados de [CGM00]. A menos de menção explícita em contrário, $\Omega$ e $\widetilde{\Omega}$ denotarão respectivamente os domínios de analiticidade de $F$ e $G$, e serão vizinhanças abertas da origem. Além disso, consideraremos $\left[\bar{x}_{-}, \bar{x}_{+}\right] \subset \Omega$, $\left[\bar{y}_{-}, \bar{y}_{+}\right] \subset \widetilde{\Omega}$ e $\bar{h}>0$ com as propriedades destacadas na seção anterior.

Observação 1.2.1 Se $F(x)=a_{2} x^{2}+O\left(x^{3}\right)$ com $a_{2}>0$ é analítica em $\Omega$, então $K$ definida por $K(x)=\frac{F(x)}{x^{2}}$, se $x \neq 0, e K(0)=a_{2}$, é também analítica em $\Omega$, pois $K$ é analítica em $\Omega-\{0\}$ e é contínua em $\Omega$. Também numa vizinhança de zero, $F(x)=\sum_{n=2}^{\infty} a_{n} x^{n}$ e portanto $K(x)=\sum_{n=0}^{\infty} a_{n+2} x^{n}$. Além disso, para $x \in\left[\bar{x}_{-}, \bar{x}_{+}\right]$temos $K(x)>0$. Concluímos que $\psi(x)=\sqrt{K(x)}$ é analítica em $\left(\bar{x}_{-}, \bar{x}_{+}\right)$.

Lema 1.2.1 Seja $F(x)=a_{2} x^{2}+O\left(x^{3}\right)$ com $a_{2}>0$, analítica em $\Omega$. Para $h \in(0, \bar{h})$ considere a função $l_{F}$ dada por (1.3). Então $l_{F}(h)=\sqrt{h} \mathcal{F}(h)$ onde $\mathcal{F}$ é analítica em zero e $\mathcal{F}(0)>0$.

Demonstração. Sejam $K$ e $\psi$ as funções definidas na observação 1.2.1. Considere a função $\phi:\left(\bar{x}_{-}, \bar{x}_{+}\right) \rightarrow(-\sqrt{\bar{h}}, \sqrt{\bar{h}})$, definida por

$$
\phi(x)=\operatorname{sgn}(x) \sqrt{F(x)} .
$$

Essa função é inversível e já que $\phi(0)=0$ e $\phi(x)=\frac{x}{|x|} \sqrt{x^{2} K(x)}=x \sqrt{K(x)}=x \psi(x)$ em $\left(\bar{x}_{-}, \bar{x}_{+}\right)-\{0\}$, resulta que $\phi$ é analítica em $\left(\bar{x}_{-}, \bar{x}_{+}\right)$e que $\phi^{\prime}(0)=\sqrt{a_{2}}>0$. Também $\phi^{\prime}(x)=\frac{F^{\prime}(x)}{2 \sqrt{F(x)}}>0 \mathrm{em}\left(0, \bar{x}_{+}\right)$e $\phi^{\prime}(x)=\frac{-F^{\prime}(x)}{2 \sqrt{F(x)}}>0$ em $\left(\bar{x}_{-}, 0\right)$. Logo $\phi^{\prime}(x)>0 \mathrm{em}$ $\left(\bar{x}_{-}, \bar{x}_{+}\right)$. Assim, $\phi$ é inversível e sua inversa é analítica em $(-\sqrt{\bar{h}}, \sqrt{\bar{h}})$. 
Se $x_{+} \in\left(0, \bar{x}_{+}\right)$e $h \in(0, \bar{h})$ são tais que $F_{+}\left(x_{+}\right)=F\left(x_{+}\right)=h$ então $\phi\left(x_{+}\right)=\sqrt{h}$ e portanto $F_{+}^{-1}(h)=x_{+}=\phi^{-1}(\sqrt{h})$. Analogamente $F_{-}^{-1}(h)=x_{-}=\phi^{-1}(-\sqrt{h})$. Assim podemos escrever $l_{F}$ como

$$
l_{F}(h)=\phi^{-1}(\sqrt{h})-\phi^{-1}(-\sqrt{h})
$$

o que nos mostra que $l_{F}(h)=\sqrt{h} \mathcal{F}_{1}(h)$ em $(0, h)$ onde $\mathcal{F}_{1}(h)=\frac{\phi^{-1}(\sqrt{h})-\phi^{-1}(-\sqrt{h})}{\sqrt{h}}$ é uma função analítica em $(0, \bar{h})$.

Além disso a função

$$
I(s)=\phi^{-1}(s)-\phi^{-1}(-s)
$$

é ímpar e analítica em $(-\sqrt{\bar{h}}, \sqrt{\bar{h}})$. Então para $s$ suficientemente pequeno temos:

$$
I(s)=\phi^{-1}(s)-\phi^{-1}(-s)=\sum_{n=0}^{\infty} b_{2 n+1} s^{2 n+1}
$$

onde $b_{1}=I^{\prime}(0)=\frac{2}{\sqrt{a_{2}}}$. Portanto, para $h>0$ suficiente pequeno temos

$$
l_{F}(h)=\sum_{n=0}^{\infty} b_{2 n+1}(\sqrt{h})^{2 n+1}=\sqrt{h} \sum_{n=0}^{\infty} b_{2 n+1} h^{n}=\sqrt{h} \mathcal{F}_{2}(h)
$$

onde $\mathcal{F}_{2}$ é analítica numa vizinhança da origem.

Pelo principio do prolongamento analítico, [Lim76], [Rud76], $\mathcal{F}_{1}$ coincide $\operatorname{com} \mathcal{F}_{2}$ onde ambas estão definidas, e assim,

$$
l_{F}(h)=\sqrt{h} \mathcal{F}(h) \text { em }(0, \bar{h}),
$$

onde $\mathcal{F}$ é uma função analítica em $[0, \bar{h})$ e $\mathcal{F}(0)=b_{1}=\frac{2}{\sqrt{a_{2}}}>0$.

Definição 1.2.1 Seja $\sigma$ uma função analítica definida em alguma vizinhança $U$ de 0 com $\sigma(0)=0$. Seja $V=\{x \in U: \sigma(x) \in U\}$. Dizemos que $\sigma$ é uma involução estrita se é diferente da identidade e $\sigma(\sigma(x))=x$ para todo $x \in V$.

Observação: Se $\sigma$ é uma involução estrita, então

a) $\sigma^{\prime}(x)<0, \forall x \in U$,

b) $\sigma^{\prime}(0)=-1$,

c) $\operatorname{sgn}(x-\sigma(x))=\operatorname{sgn} x$. 
Lema 1.2.2 Seja $F(x)=a_{2} x^{2}+O\left(x^{3}\right)$ analítica em $\Omega$ com $a_{2}>0$. Para $x \in\left(\bar{x}_{-}, \bar{x}_{+}\right)$ definamos

$$
\sigma(x)=x-\operatorname{sgn}(x) l_{F}(F(x)) .
$$

Então $\sigma$ é uma involução estrita analítica tal que $F(x)=F(\sigma(x))$.

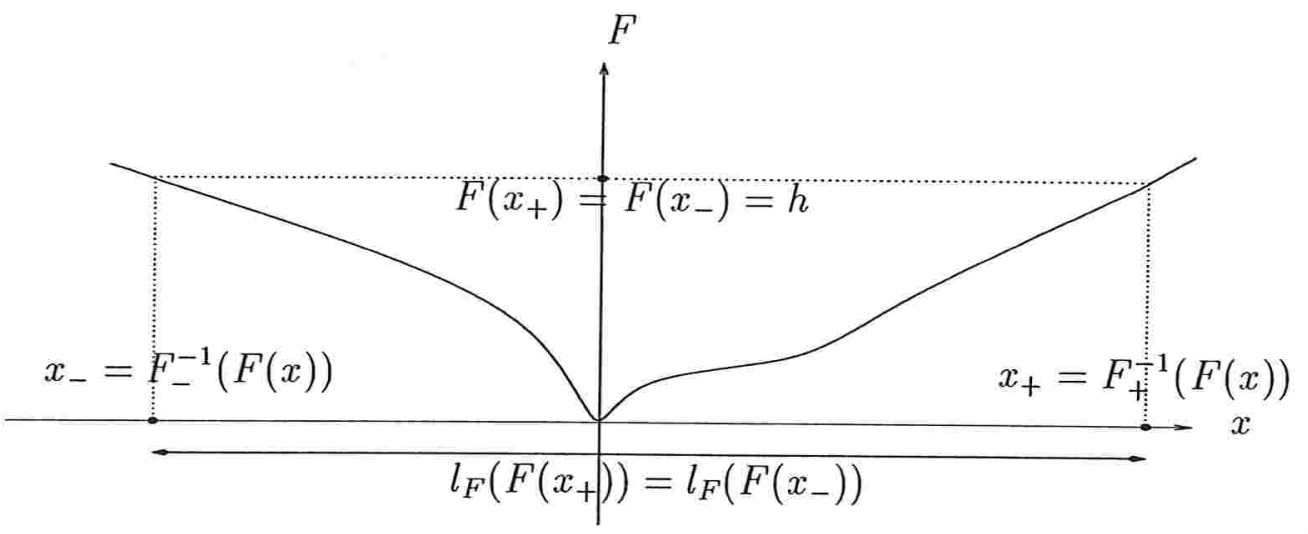

Figura 1.3: Gráfico de F

Demonstração. Pelo Lema 1.2.1 temos $l_{F}(F(x))=\sqrt{F(x)} \mathcal{F}(F(x))$, logo

$$
\sigma(x)=x-\operatorname{sgn}(x) \sqrt{F(x)} \mathcal{F}(F(x))=x-\phi(x) \mathcal{F}(F(x)),
$$

que é claramente analítica. Pela fig. 1.3 podemos observar que

$$
\sigma(x)=x-\operatorname{sgn}(x) l_{F}(F(x))= \begin{cases}x-l_{F}(F(x))<0, & \text { se } 0<x<\bar{x}_{+} \\ 0 & \text { se } x=0 \\ x+l_{F}(F(x))>0, & \text { se } \bar{x}_{-}<x<0\end{cases}
$$

Note que $\operatorname{sgn}(\sigma(x))=-\operatorname{sgn}(x) \quad$ e que $\quad \sigma(x)=x_{h-}=F_{-}^{-1}(h) \quad$ quando $x=x_{h+}=F_{+}^{-1}(h)>0$ e $\sigma(x)=x_{h+}=F_{+}^{-1}(h)$ quando $x=x_{h-}=F_{-}^{-1}(h)<0$, portanto

$$
F(\sigma(x))=F(x), \forall x \in\left[\bar{x}_{-}, \bar{x}_{+}\right]
$$

De (1.8) vem

$$
l_{F}(F(x))=l_{F}(F(\sigma(x)))
$$

e assim

$$
\sigma(\sigma(x))=\sigma(x)-\operatorname{sgn}(\sigma(x)) l_{F}(F(\sigma(x)))=\left(x-\operatorname{sgn}(x) l_{F}(F(x))\right)+\operatorname{sgn}(x) l_{F}(F(x))=x .
$$


Além disso, de (1.6) obtemos

$$
\sigma^{\prime}(x)=1-\left[\phi^{\prime}(x) \mathcal{F}(F(x))+\phi(x) \mathcal{F}^{\prime}(F(x)) F^{\prime}(x)\right]
$$

o que nos dá $\sigma^{\prime}(0)=-1$, pois $\phi^{\prime}(0)=\sqrt{a_{2}}$ e $\mathcal{F}(0)=\frac{2}{\sqrt{a_{2}}}$. Portanto $\sigma$ é uma involução estrita.

Proposição 1.2.1 Sejam $l(h)=\sqrt{h} \mathcal{L}(h)$ com $\mathcal{L}$ analítica e $\mathcal{L}(0)>0$ e uma involução estrita analítica $\sigma$. Então existe uma única função analítica $F$ da forma $F(x)=a_{2} x^{2}+O\left(x^{3}\right)$ definida numa vizinhança $W_{0}$ da origem, com $a_{2}>0$, tal que $l(h)=F_{+}^{-1}(h)-F_{-}^{-1}(h)$ para $h>0$ suficientemente pequeno, e $\sigma(x)=x-\operatorname{sgn}(x) l(F(x))$ em $W_{0}$.

Demonstração. A função $L(s)=s \mathcal{L}\left(s^{2}\right)$ é analítica e $L^{\prime}(0)=\mathcal{L}(0)>0$. Assim existe uma vizinhança $V=(-\sqrt{\bar{h}}, \sqrt{\bar{h}})$ de zero onde $L$ é estritamente crescente e admite uma inversa $L^{-1}$ analítica em $W=L(V)$. Como $L(0)=L^{-1}(0)=0$ e ambas são crescente temos

$$
\operatorname{sgn}(L(s))=\operatorname{sgn}(s) \text { e } \operatorname{sgn}\left(L^{-1}(x)\right)=\operatorname{sgn}(x) .
$$

Seja $W_{0}$ vizinhança aberta de zero tal que $x \in W_{0}$ se, e somente se, $x-\sigma(x) \in W$, isto é, $W_{0}=(I-\sigma)^{-1}(W)$, onde $I(x)=x$. Para $x \in W_{0}$ definimos

$$
F(x)=\left[L^{-1}(x-\sigma(x))\right]^{2}
$$

Claramente $F$ é analítica e $F(x) \in[0, \bar{h}), \forall x \in W_{0}$. Como $\sigma(0)=0$ e $L^{-1}(0)=0$ temos

$$
F(0)=0
$$

Além disso, $F^{\prime}(x)=2\left[L^{-1}(x-\sigma(x))\right]\left[\left(L^{-1}\right)^{\prime}(x-\sigma(x))\right]\left(1-\sigma^{\prime}(x)\right)$ e como $L^{-1}(0)=0$ temos

$$
F^{\prime}(0)=0
$$

$$
\begin{gathered}
F^{\prime \prime}(x)=2\left[\left[\left(L^{-1}\right)^{\prime}(x-\sigma(x))\right]\left(1-\sigma^{\prime}(x)\right)\right]^{2}+2\left[L^{-1}(x-\sigma(x))\right]\left[\left[\left(L^{-1}\right)^{\prime}(x-\sigma(x))\right]\left(1-\sigma^{\prime}(x)\right)\right]^{\prime} \\
F^{\prime \prime}(0)=2\left(\frac{1-\sigma^{\prime}(0)}{L^{\prime}(0)}\right)^{2}>0 \text { pois } \sigma^{\prime}(0)<0 .
\end{gathered}
$$

Então $F$ tem a forma pretendida.

Observe que como $\sigma(\sigma(x))=x$ então $\left(\sigma^{\prime}(0)\right)^{2}=1$ e como $\sigma$ é uma involução estrita analítica vem que $\sigma^{\prime}(0)=-1<0$. 
Portanto, como $l\left(k^{2}\right)=\sqrt{k^{2}} \mathcal{L}\left(k^{2}\right)=\operatorname{sgn}(k) k \mathcal{L}\left(k^{2}\right)=\operatorname{sgn}(k) L(k)$, temos que

$$
\begin{aligned}
l(F(x)) & =l\left(\left[L^{-1}(x-\sigma(x))\right]^{2}\right)=\operatorname{sgn}\left(\left[L^{-1}(x-\sigma(x))\right]\right) L\left(\left[L^{-1}(x-\sigma(x))\right]\right) \\
& =\operatorname{sgn}(x-\sigma(x))(x-\sigma(x))=\operatorname{sgn}(x)(x-\sigma(x)) .
\end{aligned}
$$

Assim, $\operatorname{sgn}(x) l(F(x))=x-\sigma(x)$ em $W_{0}$, ou seja,

$$
\sigma(x)=x-\operatorname{sgn}(x) l(F(x)) \text { em } W_{0} .
$$

Da definição de $\mathrm{F}$ temos

$$
F(\sigma(x))=\left[L^{-1}(\sigma(x)-\sigma(\sigma(x))]^{2}=\left[L^{-1}(\sigma(x)-x)\right]^{2} \stackrel{(*)}{=}\left[L^{-1}(x-\sigma(x))\right]^{2}=F(x) .\right.
$$

(Note que a igualdade em (*) ocorre porque $L^{-1}$ é ímpar em $W$, já que $L$ é ímpar em $V$.)

Portanto, denotando por $x_{h+}=F_{+}^{-1}(h)$ e $x_{h-}=F_{-}^{-1}(h)$ temos $\sigma\left(x_{h+}\right)=x_{h-}$, e observando que $l(F(x))=\operatorname{sgn}(x-\sigma(x))(x-\sigma(x))=(x-\sigma(x))$, se $x \geq 0$, vem que

$$
l(h)=l\left(F\left(x_{h+}\right)\right)=x_{h+}-\sigma\left(x_{h+}\right)=F_{+}^{-1}(h)-F_{-}^{-1}(h) .
$$

Note que se $l(F(x))=l(\widetilde{F}(x))$ então $L(\sqrt{F(x)})=L(\sqrt{\widetilde{F}(x)})$, logo a unicidade da $F$ decorre do fato de $L$ ser estritamente crescente em $V$.

Relembremos que a convolução entre duas funções $\varphi_{1}$ e $\varphi_{2}$ é dada por

$$
\left(\varphi_{1} * \varphi_{2}\right)(h)=\int_{0}^{h} \varphi_{1}(z) \varphi_{2}(h-z) d z=\left(\varphi_{2} * \varphi_{1}\right)(h), \quad \text { (veja }[\text { Kre88]). }
$$

Lema 1.2.3 Sejam $l(h)=\sqrt{h} \mathcal{L}(h), \mathcal{L}$ analítica com $\mathcal{L}(0)>0$, e $L(y)=y \mathcal{L}\left(y^{2}\right)$. Dadas $G$ analítica em zero, da forma $G(y)=b_{2} y^{2}+O\left(y^{3}\right)$ com $b_{2}>0$ tal que $l_{G}=l$, e $\sigma(y)=y-\operatorname{sgn}(y) l(G(y))$, então $G(y)=\left[L^{-1}(y-\sigma(y))\right]^{2}$ numa vizinhança da origem.

Demonstração. Pelo Lema 1.2.2, $\sigma$ é uma involução estrita analítica. Então pela Proposição 1.2.1 decorre que existe uma única função analítica $\widetilde{G}$ definida numa vizinhança $W_{0}$ da origem, da forma $\widetilde{G}(y)=\tilde{b}_{2} y^{2}+O\left(y^{3}\right)$ e tal que $l(h)=\widetilde{G}_{+}^{-1}(h)-\widetilde{G}_{-}^{-1}(h)=l_{\widetilde{G}}(h)$ para $h>0$ suficientemente pequeno e $\sigma(y)=y-\operatorname{sgn}(y) l(\widetilde{G}(y))$ em $W_{0}$.

Da unicidade de $\widetilde{G}$ e de nossas hipóteses segue que $G=\widetilde{G}$ onde ambas estiverem definidas.

Da construção de $\widetilde{G}$ na prova da Proposição 1.2.1 decorre que $G(y)=\widetilde{G}(y)=\left[L^{-1}(y-\sigma(y))\right]^{2}$ numa vizinhança da origem. 
Vamos agora estudar a função período $T$. O valor $T(h)$ é o período da órbita contida na curva de nível $H(x, y)=h$.

O seguinte Lema nos será muito útil nesse estudo.

Lema 1.2.4 Sejam $l_{F}(z)=\sqrt{z} \mathcal{F}(z)$ e $l_{G}(z)=\sqrt{z} \mathcal{G}(z)$ em $[0, \bar{h}]$, onde $\mathcal{F}$ e $\mathcal{G}$ são funções analíticas em $[0, \bar{h}]$. Então $l_{F} * l_{G}$ é derivável e vale:
(a) $\frac{d}{d h}\left(l_{F} * l_{G}\right)(h)=\left(l_{F}^{\prime} * l_{G}\right)(h) \quad e$
(b) $\frac{d}{d h}\left(l_{F}^{\prime} * l_{G}\right)(h)=\frac{d}{d h}\left(l_{G} * l_{F}^{\prime}\right)(h)=\left(l_{F}^{\prime} * l_{G}^{\prime}\right)(h)$

Demonstração. (a) Definamos $\delta(h, z)=l_{F}(z) l_{G}(h-z)$ em $[0, \bar{h}] \times[0, \bar{h}]$.

Então $\quad\left(l_{F} * l_{G}\right)(h)=\int_{0}^{h} \delta(h, z) d z=\int_{0}^{\bar{h}} \delta_{1}(h, z) d z$ onde $\delta_{1}(h, z)=\delta(h, z) \chi_{[0, h]}(z)$

Para $h_{0} \in[0, \bar{h}]$, a função $\delta_{1}\left(z, h_{0}\right)$ é integrável em $[0, \bar{h}]$ pois $\delta$ é contínua.

Existe a derivada parcial $\frac{\partial}{\partial h} \delta_{1}(h, z)$ para todo $(h, z) \in[0, \bar{h}] \times[0, \bar{h}]$, com $z \neq h$ e vale $\frac{\partial}{\partial h} \delta_{1}(h, z)=l_{F}(z) \frac{\partial}{\partial h}\left[l_{G}(h-z)\right] \chi_{[0, h]}(z)=\frac{1}{2} \sqrt{z} \mathcal{F}(z) \frac{\mathcal{G}(h-z)+2(h-z) \mathcal{G}^{\prime}(h-z)}{\sqrt{h-z}} \chi_{[0, h]}(z)$. E mais, $\frac{\partial}{\partial h} \delta_{1}(h, z)$ é integrável e como $\mathcal{F}, \mathcal{G}$ e $\mathcal{G}^{\prime}$ são contínuas, existe $K>0$ tal que

$$
\begin{aligned}
\left|\frac{\partial}{\partial h} \delta_{1}(h, z)\right| & \leq\left|\frac{\partial}{\partial h} \delta(h, z)\right|=\left|l_{F}(z) \frac{\partial}{\partial h} l_{G}(h-z)\right|=\left|\sqrt{z} \mathcal{F}(z) \frac{\mathcal{G}(h-z)+2(h-z) \mathcal{G}^{\prime}(h-z)}{2 \sqrt{h-z}}\right| \\
& \leq\left|\frac{\sqrt{z} \mathcal{F}(z) \mathcal{G}(h-z)}{2 \sqrt{h-z}}\right|+\left|\frac{\sqrt{z} \mathcal{F}(z) 2(h-z) \mathcal{G}^{\prime}(h-z)}{2 \sqrt{h-z}}\right| \leq \frac{K}{\sqrt{h-z}}=\Psi(z),
\end{aligned}
$$

que é integrável em $[0, \bar{h}]$.

O resultado segue do Teorema da Convergência Dominada de Lebesgue, (ver [Bar95], $[\operatorname{Rud} 76])$.

(b) Definamos $\rho(h, z)=l_{F}^{\prime}(z) l_{G}(h-z)=\frac{\mathcal{F}(z)+2 z \mathcal{F}^{\prime}(z)}{2 \sqrt{z}} \sqrt{h-z} \mathcal{G}(h-z)$.

Note que $\rho$ é produto de função integrável $\frac{1}{\sqrt{z}}$ por função limitada em $[0, \bar{h}] \times[0, \bar{h}]$, logo $\rho$ é integrável na variável $z$ em $[0, \bar{h}]$ para cada $h \in[0, \bar{h}]$. Assim para $h_{0} \in[0, \bar{h}]$ a função $\rho_{1}\left(h_{0}, z\right)=\rho\left(h_{0}, z\right) \chi_{\left[0, h_{0}\right]}(z)$ é integrável em $[0, \bar{h}]$.

Existe a derivada parcial $\frac{\partial}{\partial h} \rho_{1}(h, z)$ para todo $(h, z) \in[0, \bar{h}] \times[0, \bar{h}]$ tal que $h \neq z$ e vale $\frac{\partial}{\partial h} \rho_{1}(h, z)=\left(\frac{\mathcal{F}(z)+2 z \mathcal{F}^{\prime}(z)}{2 \sqrt{z}}\right)\left(\frac{\mathcal{G}(h-z)+2(h-z) \mathcal{G}^{\prime}(h-z)}{2 \sqrt{h-z}}\right) \chi_{[0, h]}(z)$ se $h \neq z$. 
Além disso, $\frac{\partial}{\partial h} \rho_{1}(h, z)$ é integrável e como $\mathcal{F}, \mathcal{G}, \mathcal{F}^{\prime}$ e $\mathcal{G}^{\prime}$ são contínuas, existe $K>0$ tal que

$$
\begin{aligned}
\left|\frac{\partial}{\partial h} \rho_{1}(h, z)\right| & \leq\left|\frac{\partial}{\partial h} \rho(h, z)\right|=\left|\frac{\mathcal{F}(z)+2 z \mathcal{F}^{\prime}(z)}{2 \sqrt{z}}\right|\left|\frac{\mathcal{G}(h-z)+2(h-z) \mathcal{G}^{\prime}(h-z)}{2 \sqrt{h-z}}\right| \\
& \leq\left(\left|\frac{\mathcal{F}(z)}{2 \sqrt{z}}\right|+\left|\frac{2 z \mathcal{F}^{\prime}(z)}{2 \sqrt{z}}\right|\right)\left(\left|\frac{\mathcal{G}(h-z)}{2 \sqrt{h-z}}\right|+\left|\frac{2(h-z) \mathcal{G}^{\prime}(h-z)}{2 \sqrt{h-z}}\right|\right) \\
& \leq \frac{K_{1}}{\sqrt{z}} \frac{K_{2}}{\sqrt{h-z}}=\frac{K}{\sqrt{z} \sqrt{h-z}}=\Psi_{1}(z) \text { que é integrável em }[0, \bar{h}] .
\end{aligned}
$$

O resultado segue de modo análogo ao ítem $(a)$.

Proposição 1.2.2 Seja T a função periodo associada a (1.2). Então

$$
T(h)=\left(l_{F}^{\prime} * l_{G}^{\prime}\right)(h)=\frac{d}{d h}\left(l_{F}^{\prime} * l_{G}\right)(h)=\frac{d^{2}}{d h^{2}}\left(l_{F} * l_{G}\right)(h)
$$

onde $l_{F}$ e $l_{G}$ estão definidas em (1.3).

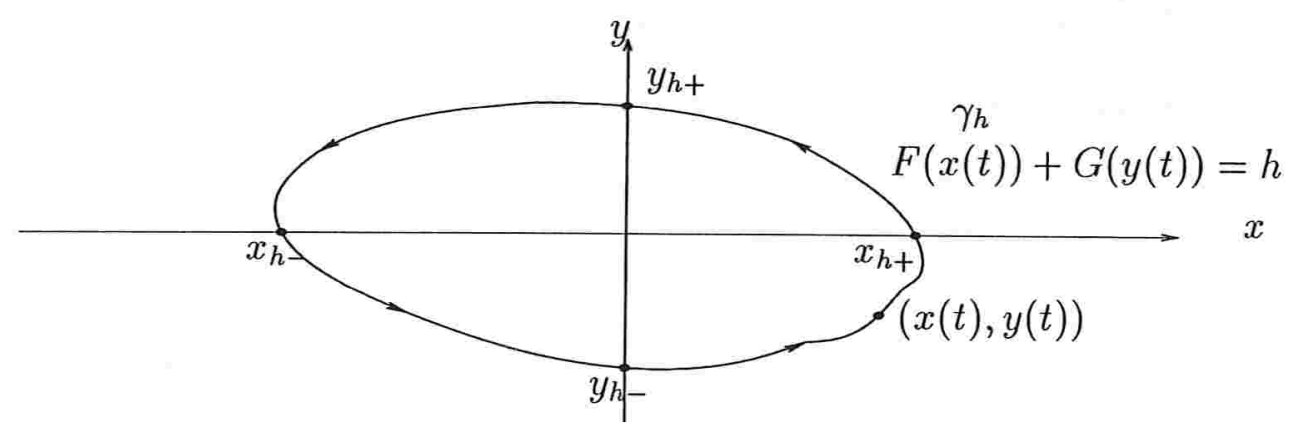

Figura 1.4: Órbita do sistema (1.2) contida na curva de nível $H(x, y)=h$

Demonstração. Sejam $x_{h-}, x_{h+}, y_{h-}, y_{h+}$, como apresentadas na seção anterior para a órbita $\gamma_{h}$ onde $h \in(0, \bar{h}]$.

Seja $(x(t), y(t))$ a solução de (1.2) tal que $(x(0), y(0))=\left(x_{h_{+}}, 0\right)$, e seja $t_{1}>0$ o primeiro instante em que $x(t)=x_{h-}$ e $y(t)=0$. Então $0<y(t)<\bar{y}_{+}, \forall t \in\left(0, t_{1}\right)$. Seja $t_{2}>0$ tal que $t_{1}+t_{2}=T(h)$. Então $\bar{y}_{-}<y(t)<0, \forall t \in\left(t_{1}, t_{1}+t_{2}\right)=\left(t_{1}, T(h)\right)$.

Como

$$
\frac{d x}{d t}(t)=-G^{\prime}(y(t)) \text { e } \frac{d y}{d t}(t)=F^{\prime}(x(t))
$$


temos $x(t)$ inversível em $\left(0, t_{1}\right)$, pois para todo $t$ nesse intervalo temos $G^{\prime}(y(t))>0$, já que $0<y(t)<\bar{y}_{+}$. Decorre que a inversa $t(x)$ satisfaz

$$
\frac{d t}{d x}(x)=-\frac{1}{G^{\prime}(y(t(x)))} \forall x \in\left(x_{h-}, x_{h+}\right)
$$

$\log 0$

$$
\begin{aligned}
0-t_{1} & =\int_{x_{h-}}^{x_{h+}} t^{\prime}(x) d x=-\int_{x_{h-}}^{x_{h+}} \frac{1}{G^{\prime}(y(t(x)))} d x, \\
\text { isto é, } t_{1} & =\int_{x_{h-}}^{x_{h+}} \frac{1}{G^{\prime}(y(t(x)))} d x=\int_{x_{h-}}^{x_{h+}} \frac{1}{G^{\prime}\left(G_{+}^{-1}(h-F(x))\right.} d x,
\end{aligned}
$$

pois para $t(x) \in\left(0, t_{1}\right)$ temos $y(t(x))>0$ e como $F(x(t))+G(y(t))=h, \forall t \in\left(0, t_{1}\right)$ vem que $y(t(x))=G_{+}^{-1}(h-F(x))$.

Então

$$
\begin{aligned}
t_{1} & =\int_{x_{h-}}^{x_{h+}} \frac{1}{G^{\prime}\left(G_{+}^{-1}(h-F(x))\right.} d x \\
& =\int_{x_{h-}}^{0} \frac{1}{G^{\prime}\left(G_{+}^{-1}(h-F(x))\right.} d x+\int_{0}^{x_{h+}} \frac{1}{G^{\prime}\left(G_{+}^{-1}(h-F(x))\right.} d x
\end{aligned}
$$

e usando as mudanças de variáveis $x=F_{-}^{-1}(z)$ para $z$ em $[0, h]$ e $x$ em $\left[x_{h-}, 0\right]$, e $x=F_{+}^{-1}(z)$ para $z$ em $[0, h]$ e $x$ em $\left[0, x_{h+}\right]$, temos

$$
\begin{aligned}
t_{1} & =\int_{h}^{0} \frac{1}{F^{\prime}\left(F_{-}^{-1}(z)\right)} \frac{1}{G^{\prime}\left(G_{+}^{-1}(h-z)\right)} d z+\int_{0}^{h} \frac{1}{F^{\prime}\left(F_{+}^{-1}(z)\right)} \frac{1}{G^{\prime}\left(G_{+}^{-1}(h-z)\right)} d z \\
& =\int_{0}^{h} \frac{1}{G^{\prime}\left(G_{+}^{-1}(h-z)\right)}\left[\frac{1}{F^{\prime}\left(F_{+}^{-1}(z)\right)}-\frac{1}{F^{\prime}\left(F_{-}^{-1}(z)\right)}\right] d z \\
& =\int_{0}^{h} \frac{\left(F_{+}^{-1}\right)^{\prime}(z)-\left(F_{-}^{-1}\right)^{\prime}(z)}{G^{\prime}\left(G_{+}^{-1}(h-z)\right)} d z .
\end{aligned}
$$

Analogamente,

$$
t_{2}=-\int_{0}^{h} \frac{\left(F_{+}^{-1}\right)^{\prime}(z)-\left(F_{-}^{-1}\right)^{\prime}(z)}{G^{\prime}\left(G_{-}^{-1}(h-z)\right)} d z
$$

e portanto

$$
\begin{aligned}
T(h) & =t_{1}+t_{2}=\int_{0}^{h} l_{F}^{\prime}(z)\left[\frac{1}{G^{\prime}\left(G_{+}^{-1}(h-z)\right)}-\frac{1}{G^{\prime}\left(G_{-}^{-1}(h-z)\right)}\right] d z \\
& =\int_{0}^{h} l_{F}^{\prime}(z)\left[\left(G_{+}^{-1}\right)^{\prime}(h-z)-\left(G_{-}^{-1}\right)^{\prime}(h-z)\right] d z=\int_{0}^{h} l_{F}^{\prime}(z) l_{G}^{\prime}(h-z) d z \\
& =\left(l_{F}^{\prime} * l_{G}^{\prime}\right)(z) .
\end{aligned}
$$


As outras duas igualdades em 1.9 seguem do Lema 1.2.4.

Observação 1.2.2 Se $F(x)=a_{2} x^{2}+O\left(x^{3}\right)$ é analítica em zero com $a_{2}>0$ decorre do Lema 1.2.1 que $l_{F}^{\prime}(h)=\frac{\mathcal{F}(h)}{2 \sqrt{h}}+\sqrt{h} \mathcal{F}^{\prime}(h)=\frac{\frac{1}{2} \mathcal{F}(h)+h \mathcal{F}^{\prime}(h)}{\sqrt{h}}$ onde $\mathcal{F}$ é analítica em $[0, \bar{h})$ e $\mathcal{F}(0)>0$. Assim,

$$
A(h)=\frac{1}{2} \mathcal{F}(h)+h \mathcal{F}^{\prime}(h)
$$

é analítica e $A(0)=\frac{1}{2} \mathcal{F}(0)>0$. Do mesmo modo, se $G(y)=b_{2} y^{2}+O\left(y^{3}\right)$ é analítica em zero com $b_{2}>0$ teremos $l_{G}^{\prime}(h)=\frac{\frac{1}{2} \mathcal{G}(h)+h \mathcal{G}^{\prime}(h)}{\sqrt{h}}$ onde $\mathcal{G}$ é analítica em $[0, \bar{h})$, e também

$$
B(h)=\frac{1}{2} \mathcal{G}(h)+h \mathcal{G}^{\prime}(h)
$$

é analítica e $B(0)=\frac{1}{2} \mathcal{G}(0)>0$. Com isto, (1.9) fica

$$
T(h)=\int_{0}^{h} l_{F}^{\prime}(h-z) l_{G}^{\prime}(z) d z=\int_{0}^{h} \frac{A(h-z)}{\sqrt{(h-z)}} \frac{B(z)}{\sqrt{z}} d z,
$$

$\operatorname{com} \frac{B(z)}{\sqrt{z}}=l_{G}^{\prime}(z)$

Observação 1.2.3 Se temos $F$ e $T$ dadas e queremos obter $G$, devemos olhar a expressão anterior (1.10) como uma equação integral em B(z). A solução $B$ procurada deve ser tal que a equação $l_{G}^{\prime}(z)=\frac{B(z)}{\sqrt{z}}$ tenha uma solução analítica $G(z)$ definida numa vizinhança da origem.

Nota: A equação

$$
T(h)=\int_{0}^{h} \frac{A(h-z) C(z)}{\sqrt{(h-z)}} d z
$$

é conhecida como a equação de $\mathcal{A}$ bel. Note que em (1.10) olhada como equação em $B(z)$ estamos procurando solução da equação de $\mathcal{A}$ bel que seja da forma $C(z)=\frac{B(z)}{\sqrt{h}}$.

Exemplo: No caso em que $F(x)=a_{2} x^{2}$ temos

$$
h=a_{2} x^{2} \Leftrightarrow x= \pm \frac{\sqrt{h}}{\sqrt{a_{2}}}
$$

e portanto

$$
l_{F}(h)=F_{+}^{-1}(h)-F_{-}^{-1}(h)=2 \sqrt{h} \frac{1}{\sqrt{a_{2}}} .
$$


Assim a função $\mathcal{F}$ do Lema 1.2 .1 será $\mathcal{F}(h)=\frac{2}{\sqrt{a_{2}}}$, e $A(h)=\frac{1}{2} \mathcal{F}(h)=\frac{1}{\sqrt{a_{2}}}$.

O seguinte Lema será usado neste e nos próximos capítulos.

Lema 1.2.5 Sejam $\sum_{i=0}^{\infty} a_{i} z^{i} e \sum_{j=0}^{\infty} b_{j} z^{j}$ convergentes em $(-R, R)$. Então

(a) As séries

$$
\sum_{i=0}^{\infty} a_{i} \operatorname{sen}^{2 i} \theta z^{i} e \sum_{j=0}^{\infty} b_{j} \cos ^{2 j} \theta z^{j}
$$

convergem absolutamente se $\theta \in\left[0, \frac{\pi}{2}\right]$ e $z \in(-R, R)$.

Além disso, para cada $z \in(-R, R)$ essas séries convergem uniformemente em $\left[0, \frac{\pi}{2}\right]$. (b) A série

$$
\sum_{n=0}^{\infty}\left(\sum_{i+j=n} a_{i} b_{j} \operatorname{sen}^{2 i} \theta \cos ^{2 j} \theta\right) z^{n}
$$

converge absolutamente $[$ Lim 76$]$ se $\theta \in\left[0, \frac{\pi}{2}\right]$ e $z \in(-R, R)$.

Além disso, para cada $z \in(-R, R)$ essa série converge uniformemente [Lim76] em $\left[0, \frac{\pi}{2}\right]$ e vale

$$
\sum_{n=0}^{\infty}\left(\sum_{i+j=n} a_{i} b_{j} \operatorname{sen}^{2 i} \theta \cos ^{2 j} \theta\right) z^{n}=\left(\sum_{i=0}^{\infty} a_{i} \operatorname{sen}^{2 i} \theta z^{i}\right)\left(\sum_{j=0}^{\infty} b_{j} \cos ^{2 j} \theta z^{j}\right)
$$

(c) Para cada $h \in[0, R)$ e $\alpha, \beta>-1$, as funções

$$
\begin{gathered}
W_{h, \alpha, \beta}^{k}(\theta)=\operatorname{sen}^{\alpha} \theta \cos ^{\beta} \theta \sum_{n=0}^{k}\left(\sum_{i+j=n} a_{i} b_{j} \operatorname{sen}^{2 i} \theta \cos ^{2 j} \theta\right) h^{n}, k \in \mathbb{N} \\
W_{h, \alpha, \beta}(\theta)=\operatorname{sen}^{\alpha} \theta \cos ^{\beta} \theta \sum_{n=0}^{\infty}\left(\sum_{i+j=n} a_{i} b_{j} \operatorname{sen}^{2 i} \theta \cos ^{2 j} \theta\right) h^{n},
\end{gathered}
$$

são contínuas em $\left(0, \frac{\pi}{2}\right)$ e absolutamente integráveis em $\left[0, \frac{\pi}{2}\right]$.

Além disso,

$$
W_{h, \alpha, \beta}^{k}(\theta) \rightarrow W_{h, \alpha, \beta}(\theta) \text { quando } k \rightarrow \infty
$$

para cada $\theta \in\left(0, \frac{\pi}{2}\right)$.

(d) $\left|W_{h, \alpha, \beta}^{k}(\theta)\right| \leq M_{h} \operatorname{sen}^{\alpha} \theta \cos ^{\beta} \theta, \forall \theta \in\left(0, \frac{\pi}{2}\right)$, 
onde $M_{h}=\sum_{n=0}^{\infty}\left(\sum_{i+j=n}\left|a_{i} b_{j}\right|\right) h^{n}<\infty$.

(e) Para $h \in[0, R)$ e $\alpha, \beta>-1$ temos

$$
\begin{aligned}
& \int_{0}^{\frac{\pi}{2}} \sum_{n=0}^{\infty}\left(\sum_{i+j=n} a_{i} b_{j} \operatorname{sen}^{2 i+\alpha} \theta \cos ^{2 j+\beta} \theta\right) h^{n} d \theta \\
= & \int_{0}^{\frac{\pi}{2}} W_{h, \alpha, \beta}(\theta) d \theta \\
= & \lim _{k \rightarrow \infty} \int_{0}^{\frac{\pi}{2}} W_{h, \alpha, \beta}^{k}(\theta) d \theta \\
= & \sum_{n=0}^{\infty} \int_{0}^{\frac{\pi}{2}}\left(\sum_{i+j=n} a_{i} b_{j} \operatorname{sen}^{2 i+\alpha} \theta \cos ^{2 j+\beta} \theta\right) h^{n} d \theta \\
= & \sum_{n=0}^{\infty} \sum_{i+j=n} a_{i} b_{j}\left[\int_{0}^{\frac{\pi}{2}} \operatorname{sen}^{2 i+\alpha} \theta \cos ^{2 j+\beta} \theta d \theta\right] h^{n}
\end{aligned}
$$

Demonstração. (a) As séries $\sum_{i=0}^{\infty} a_{i} z^{i}$ e $\sum_{j=0}^{\infty} b_{j} z^{j}$ convergem absolutamente se $z \in(-R, R)$, e para cada $z \in(-R, R)$,

$$
\begin{aligned}
\left|a_{i} \operatorname{sen}^{2 i} \theta z^{i}\right| & \leq\left|a_{i}\right||z|^{i}, \forall \theta \in\left[0, \frac{\pi}{2}\right] \mathrm{e} \\
\left|b_{j} \cos ^{2 j} \theta z^{j}\right| & \leq\left|b_{j}\right||z|^{j}, \forall \theta \in\left[0, \frac{\pi}{2}\right] .
\end{aligned}
$$

Logo, a afirmação segue do teste $M$ de Weierstrass.

(b) Para cada $z \in(-R, R)$ segue da convergência absoluta das séries $\sum_{i=0}^{\infty} a_{i} z^{i} \mathrm{e} \sum_{j=0}^{\infty} b_{j} z^{j}$ que a série produto

$$
\sum_{n=0}^{\infty}\left(\sum_{i+j=n}\left|a_{i}\right|\left|b_{j}\right|\right)|z|^{n} \text { converge }
$$

e vale

$$
\sum_{n=0}^{\infty}\left(\sum_{i+j=n}\left|a_{i}\right|\left|b_{j}\right|\right)|z|^{n}=\left(\sum_{i=0}^{\infty}\left|a_{i}\right||z|^{i}\right)\left(\sum_{j=0}^{\infty}\left|b_{j}\right||z|^{j}\right)
$$

Além disso,

$$
\left|\left(\sum_{i+j=n} a_{i} b_{j} \operatorname{sen}^{2 i} \theta \cos ^{2 j} \theta\right) z^{n}\right| \leq \sum_{i+j=n}\left|a_{i}\right|\left|b_{j} \| z\right|^{n}, \forall \theta \in\left[0, \frac{\pi}{2}\right],
$$

donde segue do teste $M$ de Weierstrass a convergência uniforme e absoluta [Lim76] afirmadas. 
A igualdade (1.13) segue do fato de as séries (1.11) serem absolutamente convergentes. (c) Para $h \in[0, R)$

$$
\sum_{n=0}^{\infty}\left[\sum_{i+j=n} a_{i} b_{j} \operatorname{sen}^{2 i} \theta \cos ^{2 j} \theta\right] h^{n}
$$

é contínua, pois é limite uniforme de funções contínuas por (b). Assim, $W_{h, \alpha, \beta}$ é contínua em $\left(0, \frac{\pi}{2}\right)$.

Claramente, $W_{h, \alpha, \beta}^{k}, \quad k \geq 0$, são contínuas em $\left(0, \frac{\pi}{2}\right)$, e como $W_{h, \alpha, \beta}$, são produto de funções contínuas em $\left[0, \frac{\pi}{2}\right]$ por $\operatorname{sen}^{\alpha} \theta \cos ^{\beta} \theta$.

Como $\alpha, \beta>-1, \operatorname{sen}^{\alpha} \theta \cos ^{\beta} \theta$ é absolutamente integrável em $\left[0, \frac{\pi}{2}\right]$ e o mesmo ocorre $\operatorname{com} W_{h, \alpha, \beta}$ e $\operatorname{com} W_{h, \alpha, \beta}^{k}, k \in \mathbb{N}$.

(d) Imediato.

(e) De $(c)$ e $(d)$ segue que podemos usar o Teorema da Convergência Dominada de Lebesgue para obter a segunda igualdade.

As outras igualdades são imediatas.

Proposição 1.2.3 Sejam $A$ e T funções analíticas em zero, satisfazendo $A(0)>0$ e $T(0)>0$. Então a equação integral

$$
T(h)=\int_{0}^{h} \frac{A(h-z) B(z)}{\sqrt{h-z} \sqrt{z}} d z
$$

tem uma única solução $B$ analítica em zero que satisfaz $B(0)>0$.

Observação: A unicidade no enunciado anterior significa que se $B:[0, r) \rightarrow \mathbb{R}$ e $B_{1}$ : $\left[0, r_{1}\right) \rightarrow \mathbb{R}$ são duas soluções analíticas de (1.14), então $B \equiv B_{1}$ em $[0, r) \cap\left[0, r_{1}\right)$. Demonstração. Seja $R>0$ tal que $T(z)=\sum_{n=0}^{\infty} t_{n} z^{n}$ e $A(z)=\sum_{n=0}^{\infty} a_{n} z^{n}$ em $[0, R)$.

Comecemos provando a unicidade de solução. Seja $B(z)$ uma solução analítica em $[0, r), r \leq R$, e suponhamos que $B(z)=\sum_{n=0}^{\infty} b_{n} z^{n}$ em $\left[0, \epsilon_{0}\right)$ para algum $\epsilon_{0}>0, \epsilon_{0} \leq r$. 
Por meio da mudança de variáveis $z=h \cos ^{2} t$ temos, para $h \in\left(0, \epsilon_{0}\right)$,

$$
\begin{aligned}
T(h) & =\int_{0}^{h} \frac{A(h-z)}{\sqrt{h-z}} \frac{B(z)}{\sqrt{z}} d z \\
& =2 \int_{0}^{\frac{\pi}{2}}\left(\sum_{n=0}^{\infty} a_{n}\left(h \operatorname{sen}^{2} t\right)^{n}\right)\left(\sum_{n=0}^{\infty} b_{n}\left(h \cos ^{2} t\right)^{n}\right) \frac{h \cos t \operatorname{sen} t}{\sqrt{h^{2} \operatorname{sen}^{2} t \cos ^{2} t}} d t \\
& =2 \int_{0}^{\frac{\pi}{2}}\left(\sum_{n=0}^{\infty} a_{n}\left(h \operatorname{sen}^{2} t\right)^{n}\right)\left(\sum_{n=0}^{\infty} b_{n}\left(h \cos ^{2} t\right)^{n}\right) d t .
\end{aligned}
$$

Usando o Lema 1.2.5 podemos escrever, para $h \in\left(0, \epsilon_{0}\right)$,

$$
\begin{aligned}
T(h) & =2 \int_{0}^{\frac{\pi}{2}}\left(\sum_{k=0}^{\infty}\left(\sum_{i+j=k} a_{i} b_{j} \operatorname{sen}^{2 i} t \cos ^{2 j} t\right) h^{k}\right) d t \\
& =2 \sum_{k=0}^{\infty}\left(\sum_{i+j=k} a_{i} b_{j} \int_{0}^{\frac{\pi}{2}} \operatorname{sen}^{2 i} t \cos ^{2 j} t d t\right) h^{k}=\sum_{k=0}^{\infty}\left(\sum_{i+j=k} a_{i} b_{j} I_{i, j}\right) h^{k}
\end{aligned}
$$

onde $I_{i, j}=2 \int_{0}^{\frac{\pi}{2}} \operatorname{sen}^{2 i} t \cos ^{2 j} t d t$

Como $T(h)=\sum_{n=0}^{\infty} t_{n} h^{n}$ para $h \in\left(0, \epsilon_{0}\right) \subset[0, R)$, temos que

$$
t_{n}=\sum_{i+j=n} a_{i} b_{j} I_{i, j}, \forall n \in \mathbb{N}
$$

Como $a_{0}=A(0) \neq 0$ e $I_{0, n} \neq 0, \forall n \in \mathbb{N}$, (1.17) é um sistema linear triangular em $b_{n}$ em que os elementos da diagonal principal são todos diferentes de zero, isto é, (1.17) é um sistema linear em $b_{i}$ possível e determinado.

Assim, se $B_{1}(z), z \in\left[0, r_{1}\right)$, for outra solução analítica, sua série de potências numa vizinhança de zero será igual à de $B$. Portanto, pelo princípio do prolongamento analítico, [Lim76], [Rud76], $B_{1} \equiv B$ em $[0, r) \cap\left[0, r_{1}\right)$.

Para provarmos a existência de solução $B$ definida numa vizinhança de zero, basta mostrarmos que a série $\sum_{n=0}^{\infty} b_{n} z^{n}$, onde $\left(b_{n}\right)$ é determinada por $t_{n}=\sum_{i+j=n} a_{i} b_{j} I_{i, j}, n \in \mathbb{N}$ converge em alguma vizinhança de zero, pois então, do Lema 1.2.5 decorrerá que as passagens em (1.15) e (1.16) serão satisfeitas.

Suponhamos sem perda de generalidade que $a_{0}=1$.

Seja $C_{0}>\frac{1}{R}$. Então $\sum_{n=0}^{\infty} \frac{\left|a_{n}\right|}{C_{0}^{n}}$ e $\sum_{n=0}^{\infty} \frac{4\left|t_{n}\right|}{C_{0}^{n}}$ convergem, e portanto, existe $N \in \mathbb{N}$ tal que $\left|a_{n}\right| \leq C_{0}^{n}$ e $4\left|t_{n}\right| \leq C_{0}^{n}, \forall n>N$. 
Tomando $C=\max \left\{C_{0}, 1,\left|a_{1}\right|,\left|a_{2}\right|^{\frac{1}{2}}, \cdots,\left|a_{N}\right|^{\frac{1}{N}}, 4\left|t_{0}\right|, 4\left|t_{1}\right|,\left(4\left|t_{2}\right|\right)^{\frac{1}{2}}, \cdots,\left(4\left|t_{n}\right|\right)^{\frac{1}{N}}\right\}$ tere$\operatorname{mos} C^{n+1} \geq C^{n} \geq C_{0}^{n}, \forall n>N$, e portanto $\left|a_{n}\right| \leq C^{n}$ e $4\left|t_{n}\right| \leq C^{n+1}, \forall n \in \mathbb{N}$.

Afirmamos que $\quad\left|b_{k}\right| \leq(k+1) C^{k+1}, \forall k \in \mathbb{N}$

Faremos a prova de (1.18) por indução sobre $k$.

Para $k=0$ temos, por (1.17), $b_{0}=\frac{t_{0}}{a_{0} I_{0,0}}=\frac{t_{0}}{I_{0,0}}$. Como $I_{0,0}=\pi$ (Fato A.0.2) temos $\left|b_{0}\right| \leq\left|t_{0}\right| \leq C$.

Para $k=1$ temos, por (1.17), $b_{1}=\frac{t_{1}-a_{1} b_{0} I_{1,0}}{I_{0,1}}$, e portanto

$$
\left|b_{1}\right| \leq \frac{\left|t_{1}\right|+\left|a_{1}\right|\left|b_{0}\right| I_{1,0}}{I_{0,1}} \leq \frac{C^{2}}{4 I_{0,1}}+C^{2} \frac{I_{1,0}}{I_{0,1}} .
$$

Como $I_{1,0}=I_{0,1} \geq \frac{\pi}{2}$ (Fatos A.0.1 e A.0.2) obtemos $\left|b_{1}\right| \leq \frac{C^{2}}{2 \pi}+C^{2} \leq 2 C^{2}$.

Suponhamos a desigualdade válida para $k=0,1, \cdots, n-1$ onde $n \geq 2$.

Então por (1.17) temos

$$
\begin{aligned}
b_{n} & =\frac{t_{n}-a_{1} b_{n-1} I_{1, n-1}-\cdots-a_{n-1} b_{1} I_{n-1,1}-a_{n} b_{0} I_{n, 0}}{I_{0, n}} \\
\left|b_{n}\right| & \leq \frac{\left|t_{n}\right|+\left|a_{1}\right|\left|b_{n-1}\right|\left|I_{1, n-1}\right|+\cdots+\left|a_{n-1}\right|\left|b_{1}\right|\left|I_{n-1,1}\right|+\left|a_{n} b_{0}\right|\left|I_{n, 0}\right|}{I_{0, n}} \\
& \leq \frac{\frac{C^{n+1}}{4}+C n C^{n} I_{1, n-1}+C^{2}(n-1) C^{n-1} I_{2, n-2}+\cdots+C^{n} C I_{n, 0}}{I_{0, n}} \\
& =C^{n+1}\left[\frac{1}{4 I_{0, n}}+\frac{n I_{1, n-1}+(n-1) I_{2, n-2}+\cdots+2 I_{n-1,1}}{I_{0, n}}+\frac{I_{n, 0}}{I_{0, n}}\right]
\end{aligned}
$$

Agora usando que para $n \geq 2 I_{0, n} \geq \frac{\pi}{2 n}$ (Fato A.0.2), $I_{n, 0}=I_{0, n}$ (Fato A.0.1), $I_{j, n-j} \leq I_{1, n-1}$ se $1 \leq j \leq n-1$ (Fato A.0.5) e $I_{1, n-1}=\frac{1}{2 n-1} I_{0, n}$ (Fato A.0.6) vem que

$$
\left|b_{n}\right| \leq\left[\frac{n}{2}+\frac{1}{2 n-1}[n+(n-1)+\cdots+2]+1\right]=C^{n+1}\left[\frac{n}{2}+\frac{(n-1)(n+2)}{2(2 n-1)}+1\right]
$$

Como $n \geq 2$ temos $\frac{n-1}{2 n-1} \leq \frac{n-1}{2 n-2}=\frac{1}{2}$, e portanto

$$
\left|b_{n}\right| \leq C^{n+1}\left[\frac{n}{2}+\frac{n+2}{4}+1\right] \leq C^{n+1}\left[\frac{n}{2}+\frac{2 n}{4}+1\right]=(n+1) C^{n+1} .
$$


Assim, estabelecemos a validade de (1.18). Deste modo, a série $\sum_{n=0}^{\infty} b_{n} z^{n}$ converge no intervalo $\left(-\frac{1}{C}, \frac{1}{C}\right)$ e $B(z)=\sum_{n=0}^{\infty} b_{n} z^{n}$ satisfaz (1.14).

Proposição 1.2.4 Seja $T$ uma função analítica em zero satisfazendo $T(0)>0 . \quad A$ equação integral

$$
T(h)=\int_{0}^{h} \frac{B(h-z)}{\sqrt{h-z}} \frac{B(z)}{\sqrt{z}} d z
$$

tem uma única solução $B$ analítica em zero que satisfaz $B(0)>0$.

Observação: A unicidade nesta Proposição tem o mesmo significado que usamos na Proposição anterior.

Demonstração. Seja $R>0$ tal que $T(z)=\sum_{n=0}^{\infty} t_{n} z^{n}$ em $[0, R)$.

Argumentando como na demonstração da Proposição anterior chegamos à seguinte relação que uma solução $B(z)=\sum_{n=0}^{\infty} b_{n} z^{n}$ deve satisfazer:

$$
t_{k}=\sum_{i+j=k} b_{i} b_{j} I_{i, j}
$$

Como antes, disto decorre a unicidade de solução.

Para mostrar a existência de solução vamos procurar majorar os coeficientes $b_{n}$ dados por (1.20). Não é restrição supor $t_{0}=\pi\left(\operatorname{logo} b_{0}=1\right)$.

Vamos mostrar que existe $C>0$ tal que $\left|t_{n}\right| \leq \frac{\pi C^{n}}{4 n^{2}}, n \geq 1$, e depois vamos provar por indução que

$$
\left|b_{k}\right| \leq \frac{C^{k}}{k+1}, k \geq 0
$$

A desigualdade sobre $\left|t_{n}\right|$ decorre do fato de que se $\sum_{n=0}^{\infty}\left|t_{n}\right| \rho^{n}$ converge para algum $\rho>0$, então $\sum_{n=0}^{\infty} 4 n^{2}\left|t_{n}\right|\left(\frac{\rho}{2}\right)^{n}$ também converge.

Vamos passar à demonstração de (1.21).

Para $k=0$ temos $\left|b_{0}\right|=1$ e portanto o resultado é verdadeiro. 
Para $k=1$ temos de (1.20), usando que $I_{1,0}=I_{0,1}$ (Fato A.0.1) e que $b_{0}=1$ obtemos, $b_{1}=\frac{t_{1}}{2 I_{0,1}}$.

Como $I_{0,1} \geq \frac{\pi}{2}$ (Fa.to A.0.2) obtemos $\left|b_{1}\right| \leq \frac{\left|t_{1}\right|}{\pi} \leq \frac{C}{4} \leq \frac{C}{2}$, e portanto vale (1.21) para $k=1$.

Agora suponhamos que (1.21) é verdadeira para $k=0,1, \cdots, n-1$, onde $n \geq 2$.

De $(1.20)$, obtemos; para $n \geq 2$,

$$
b_{n}=\frac{t_{n}-b_{1} b_{n-1} I_{1, n-1}-b_{2} b_{n-2} I_{2, n-2}-\cdots-b_{n-1} b_{1} I_{n-1,1}}{2 I_{0, n}}
$$

Logo $\left|b_{n}\right| \leq \frac{\left|t_{n}\right|+\left|b_{1}\right|\left|b_{n-1}\right| I_{1, n-1}+\left|b_{2}\right|\left|b_{n-2}\right| I_{2, n-2}+\cdots+\left|b_{n-1}\right|\left|b_{1}\right| I_{n-1,1}}{2 I_{0, n}}$

$$
\leq C^{n}\left[\frac{\frac{\pi}{4 n^{2}}+\frac{1}{2} \frac{1}{n} I_{1, n-1}+\frac{1}{3} \frac{1}{n-1} I_{2, n-2}+\cdots+\frac{1}{n} \frac{1}{2} I_{n-1,1}}{2 I_{0, n}}\right]
$$

Usando que $I_{j, n-j} \leq I_{1, n-1}$ (Fato A.0.5) e que $I_{0, n} \geq \frac{\pi}{2 n}$ (Fato A.0.2) obtemos

$$
\begin{aligned}
\left|b_{n}\right| & \leq C^{n}\left[\frac{\pi}{4 n^{2}}+\frac{1}{2} \frac{1}{n} I_{1, n-1}+\frac{1}{3} \frac{1}{n-1} I_{2, n-2}+\cdots+\frac{1}{n} \frac{1}{2} I_{n-1,1}\right] \frac{1}{2 I_{0, n}} \\
& \leq C^{n}\left[\frac{1}{4 n}+\left(\frac{1}{2} \frac{1}{n}+\frac{1}{3} \frac{1}{n-1}+\cdots+\frac{1}{n} \frac{1}{2}\right) \frac{I_{1, n-1}}{2 I_{0, n}}\right] \\
& \leq C^{n}\left[\frac{1}{4 n}+\left(\frac{1}{2 n}+\frac{1}{2 n}+\cdots+\frac{1}{2 n}\right) \frac{I_{1, n-1}}{2 I_{0, n}}\right] \\
& =C^{n}\left[\frac{1}{4 n}+\frac{(n-1)}{4 n} \frac{I_{1, n-1}}{I_{0, n}}\right] .
\end{aligned}
$$

Agora usando que $I_{1, n-1}=\frac{1}{2 n-1} I_{0, n}$ (Fato A.0.6) obtemos

$$
\left|b_{n}\right| \leq C^{n}\left[\frac{1}{4 n}+\frac{n-1}{4 n(2 n-1)}\right] \leq \frac{C^{n}}{n+1}
$$

pois $\frac{1}{4 n}+\frac{n-1}{4 n(2 n-1)} \leq \frac{1}{4 n}+\frac{n-1}{4 n(n-1)} \leq \frac{1}{2 n} \leq \frac{1}{n+1}$, já que $n \geq 2$.

Assim vale (1.21). Conseqüentemente, $B(z)=\sum_{n=0}^{\infty} b_{n} z^{n}$ é solução analítica de (1.19) em $\left[0, \frac{1}{C}\right)$ 


\subsection{Principais Resultados}

No que segue, $\bar{x}_{-}, \bar{x}_{+}$e $\bar{h}$ serão valores satisfazendo as propriedades destacados na seção 1.1 .

Teorema 1.3.1 Sejam $T$ e F funções analíticas em zero, $F(x)=a_{2} x^{2}+O\left(x^{3}\right)$, satisfazendo $T(0)>0$ e $a_{2}>0$. Seja $l_{F}(h)=F_{+}^{-1}(h)-F_{-}^{-1}(h), h \in[0, \bar{h}[$. Então as seguintes afirmações são verdadeiras:

(i) Existe uma única $l(h)=\sqrt{h} \mathcal{L}(h)$ com $\mathcal{L}$ analítica em zero e $\mathcal{L}(0)>0$ tal que se $G$ é analítica em zero, tem mínimo não degenerado na origem e satisfaz $l_{G}=l$, então a origem de (1.2) é um centro com função período $T$.

(ii) Além disso, o conjunto das funções $G$ analíticas em zero que tem mínimo não degenerado na origem e satisfazem $l_{G}=l$ está em correspondência biunívoca com o conjunto $\Sigma$ das involuções estritas analíticas. Mais concretamente, para cada $\sigma \in \Sigma$ a função correspondente $G$ é dada por $G(y)=\left[L^{-1}(y-\sigma(y))\right]^{2}$ onde $L(y)=y \mathcal{L}\left(y^{2}\right)$, com $\mathcal{L}$ dada em $(i)$.

Demonstração. (i) T é analítica e $T(0)>0$; além disso pelo Lema 1.2.1, $l_{F}(h)=\sqrt{h} \mathcal{F}(h)$, o que nos dá $l_{F}^{\prime}(h)=\frac{\frac{1}{2} \mathcal{F}(h)+h \mathcal{F}^{\prime}(h)}{\sqrt{h}}$. Ainda pelo Lema, $\mathcal{F}(0)>0$. Assim, $A(h)=\frac{1}{2} \mathcal{F}(h)+h \mathcal{F}^{\prime}(h)$ satisfaz $A(0)=\frac{1}{2} \mathcal{F}(0)>0$. Nestas condições a Proposição 1.2.3 nos diz que existe uma única função $B$ analítica em zero tal que $B(0)>0$ e que é solução da equação integral

$$
T(h)=\int_{0}^{h} \frac{A(h-z)}{\sqrt{h-z}} \frac{B(z)}{\sqrt{z}} d z .
$$

Mostraremos que $B$ pode ser escrita de modo único como

$$
B(z)=\frac{1}{2} \mathcal{L}(z)+z \mathcal{L}^{\prime}(z)
$$

com $\mathcal{L}$ analítica em zero.

Como $B$ é analítica em zero, $B(z)=\sum_{n=0}^{\infty} b_{n} z^{n}$ numa vizinhança de zero, e $B(0)=b_{0}>0$ 
Suponha que exista $\mathcal{L}$ analítica em zero escrita na forma $\mathcal{L}(z)=\sum_{n=0}^{\infty} c_{n} z^{n}$ numa vizinhança de zero que satisfaz (1.23). Então

$$
\begin{aligned}
\sum_{n=0}^{\infty} b_{n} z^{n} & =\frac{1}{2} \sum_{n=0}^{\infty} c_{n} z^{n}+z \sum_{n=0}^{\infty}(n+1) c_{n+1} z^{n} \\
& =\frac{1}{2} \sum_{n=0}^{\infty} c_{n} z^{n}+\sum_{n=1}^{\infty} n c_{n} z^{n} \\
& =\frac{c_{0}}{2}+\sum_{n=1}^{\infty}\left(n+\frac{1}{2}\right) c_{n} z^{n}
\end{aligned}
$$

ou seja, $b_{0}=\frac{c_{0}}{2}$ e $b_{n}=\left(n+\frac{1}{2}\right) c_{n}$ para $n \geq 1$.

Isto mostra a unicidade de $\mathcal{L}$ analítica em zero satisfazendo (1.23).

A única candidata a $\mathcal{L}$ é dada por $\mathcal{L}(z)=\sum_{n=0}^{\infty} c_{n} z^{n}=2 b_{0}+\sum_{n=1}^{\infty} \frac{2 b_{n}}{2 n+1} z^{n}$.

Como $\left|c_{n}\right|=\left|\frac{2 b_{n}}{2 n+1}\right| \leq\left|b_{n}\right|$, a série $\sum_{n=0}^{\infty} c_{n} z^{n}$ converge onde a série $\sum_{n=0}^{\infty} b_{n} z^{n}$ converge. Portanto $\mathcal{L}$ dada acima está bem definida e é analítica numa vizinhança da origem, e satisfaz (1.23). Também, $\mathcal{L}(0)=2 B(0)>0$.

Definamos $l(h)=\sqrt{h} \mathcal{L}(h)$.

Seja $G$ analítica em zero com mínimo não degenerado na origem verificando $l_{G}=l$. $\operatorname{Assim} l_{G}^{\prime}(h)=\frac{\mathcal{L}(h)+2 h \mathcal{L}^{\prime}(h)}{2 \sqrt{h}}=\frac{B(h)}{\sqrt{h}}$.

Usando

$$
\begin{gathered}
l_{F}^{\prime}(h)=\frac{\frac{1}{2} \mathcal{F}(h)+h \mathcal{F}^{\prime}(h)}{\sqrt{h}} \text { e } A(h)=\frac{1}{2} \mathcal{F}(h)+h \mathcal{F}^{\prime}(h) \text { em (1.22), obtemos: } \\
T(h)=\int_{0}^{h} l_{F}^{\prime}(h-z) l_{G}^{\prime}(z) d z
\end{gathered}
$$

Considere o sistema (1.2) com função de Hamilton $H(x, y)=F(x)+G(y)$. É claro que $H$ tem um mínimo em $(0,0)$ e portanto a origem é um centro para (1.2).

Também é claro que nesse caso $T$ é a função Período.

Com isto provamos a existência de $l$ satisfazendo $(i)$.

Suponhamos agora que exista $l_{1}(h)=\sqrt{h} \mathcal{L}_{1}(h), \mathcal{L}_{1}$ analítica em zero com $\mathcal{L}_{1}(0)>0$, tal que para toda $G$ analítica com mínimo não degenerado na origem tal que $l_{G}(h)=l_{1}(h)$ a origem de (1.2) seja um centro de período $T$. 
Então

$$
l_{G}^{\prime}(h)=\frac{\mathcal{L}_{1}(h)+2 h \mathcal{L}_{1}^{\prime}(h)}{2 \sqrt{h}}=\frac{B_{1}(h)}{\sqrt{h}} .
$$

Claramente $B_{1}$ é analítica em zero com $B_{1}(0)=\frac{\mathcal{L}_{1}(0)}{2}>0$. Mas pela Proposição 1.2.2

$$
T(h)=\int_{0}^{h} l_{F}^{\prime}(h-z) l_{G}^{\prime}(z) d z=\int_{0}^{h} \frac{A(h-z)}{\sqrt{h-z}} \frac{B_{1}(z)}{\sqrt{z}} d z
$$

Pela unicidade dada na Proposição $1.2 .3, B_{1}=B$, e pela unicidade da representação da $B$ em termos de $\mathcal{L}$ decorre que $\mathcal{L}_{1}=\mathcal{L}$ e portanto $l_{1}=l$.

(ii) Seja $l(h)=\sqrt{h} \mathcal{L}(h)$ dada pelo ítem $(i)$.

Seja $\sigma$ uma involução estrita analítica. A Proposição 1.2.1 garante que existe uma única função $G$ analítica em zero da forma $G(y)=b_{2} y^{2}+O\left(y^{3}\right) \quad$ com $\quad b_{2}>0$ tal que $l_{G}(h)=G_{+}^{-1}(h)-G_{-}^{-1}(h)=l(h)$ e $\sigma(y)=y-\operatorname{sgn}(y) l(G(y))$. Pelo Lema 1.2.3, $G(y)=\left[L^{-1}(y-\sigma(y))\right]^{2}$.

Por outro lado, dada $G(y)=b_{2} y^{2}+O\left(y^{3}\right)$ com $b_{2}>0$, analítica em zero, tal que $l_{G}=l$, segue do Lema 1.2 .2 que $\sigma(y)=y-\operatorname{sgn}(y) l(G(y))$ é uma involução estrita analítica. Novamente pelo Lema 1.2.3, $G(y)=\left[L^{-1}(y-\sigma(y))\right]^{2}$.

Teorema 1.3.2 Considere o sistema (1.2) com $f \equiv g$. Seja $T$ uma função analítica em zero satisfazendo $T(0)>0$. Então as seguintes afirmações sẫo verdadeiras:

(i) Existe uma única $l(h)=\sqrt{h} \mathcal{L}(h)$ com $\mathcal{L}$ analítica em zero e $\mathcal{L}(0)>0$ tal que se $F \equiv G$ é analítica em zero, possui mínimo não degenerado na origem e satisfaz $l_{F} \equiv l_{G} \equiv l$, então a origem de (1.2) é um centro com função periodo $T$.

(ii) Além disso, o conjunto das funções $F$ analíticas em zero que têm mínimo não degenerado na origem, tais que a função período de (1.2) com $f=g$ é $T$ está em correspondência biunívoca com o conjunto $\Sigma$ das involuções estritas analíticas. Mais concretamente, para cada $\sigma \in \Sigma$ sua correspondente função $F$ é definida por $F(x)=\left[L^{-1}(x-\sigma(x))\right]^{2}$ onde $L(x)=x \mathcal{L}\left(x^{2}\right)$ com $\mathcal{L}$ dada em $(i)$.

Demonstração. (i) $T$ é analítica e $T(0)>0$; além disso pela Proposição 1.2.4, a equação integral

$$
T(h)=\int_{0}^{h} \frac{B(h-z)}{\sqrt{h-z}} \frac{B(z)}{\sqrt{z}} d z,
$$

possui uma única solução analítica $B$ que satisfaz $B(0)>0$. 
Como na demonstração do Teorema 1.3.1, $B$ pode ser escrita de modo único como

$$
B(z)=\frac{1}{2} \mathcal{L}(z)+z \mathcal{L}^{\prime}(z),
$$

com $\mathcal{L}$ analítica em zero e, é claro, $\mathcal{L}(0)>0$.

Definamos $l(h)=\sqrt{h} \mathcal{L}(h)$.

Sejam $F=G$ analíticas em zero, com mínimo não degenerado na origem verificando $l_{F}=l_{G}=l$. Assim

$$
\begin{aligned}
& l_{F}^{\prime}(h)=\frac{\mathcal{L}(h)+2 h \mathcal{L}^{\prime}(h)}{2 \sqrt{h}}=\frac{B(h)}{\sqrt{h}} \text { e } l_{G}^{\prime}(h)=\frac{\mathcal{L}(h)+2 h \mathcal{L}^{\prime}(h)}{2 \sqrt{h}}=\frac{B(h)}{\sqrt{h}} . \\
& \text { Logo, } T(h)=\int_{0}^{h} l_{F}^{\prime}(h-z) l_{G}^{\prime}(z) d z .
\end{aligned}
$$

Considere o sistema (1.2) com função de Hamilton $H(x, y)=F(x)+G(y)=F(x)+F(y)$. É claro que $H$ tem um mínimo estrito em $(0,0)$ e portanto a origem é um centro para (1.2), com função período $T$.

Com isto, está provado a existência de $l$.

Suponhamos agora que exista $l_{1}(h)=\sqrt{h} \mathcal{L}_{1}(h), \mathcal{L}_{1}$ analítica em zero com $\mathcal{L}_{1}(0)>0$, tal que para toda $F=G$ analítica em zero com mínimo não degenerado na origem tal que $l_{F}^{\prime}=l_{G}^{\prime}=l$ obtém-se $T$ como função período de (1.2).

Procedendo como na prova do Teorema anterior obtemos

$$
l_{F}^{\prime}(h)=l_{G}^{\prime}(h)=\frac{B_{1}(h)}{\sqrt{h}},
$$

com $B_{1}$ analítica em zero e $B_{1}(0)=\frac{\mathcal{L}_{1}(0)}{2}>0$. Pela Proposição 1.2 .2

$$
T(h)=\int_{0}^{h} l_{F}^{\prime}(h-z) l_{G}^{\prime}(z) d z=\int_{0}^{h} \frac{B_{1}(h-z)}{\sqrt{h-z}} \frac{B_{1}(z)}{\sqrt{z}} d z .
$$

Pela unicidade dada na Proposição $1.2 .4, B_{1}=B$, e pela unicidade da representação da $B$ em termos de $\mathcal{L}$ decorre que $\mathcal{L}_{1}=\mathcal{L}$ e portanto $l_{1}=l$.

(ii) Seja $l(h)=\sqrt{h} \mathcal{L}(h)$ dada pelo ítem $(i)$.

Seja $\sigma$ uma involução estrita analítica. A Proposição 1.2.1 garante que existe uma única função $F$ analítica em zero, da forma $F(x)=a_{2} x^{2}+O\left(x^{3}\right) \quad$ com $\quad a_{2}>0$ tal que $l_{F}(h)=F_{+}^{-1}(h)-F_{-}^{-1}(h)=l(h)$ e $\sigma(x)=x-\operatorname{sgn}(x) l(G(x))$. Pelo Lema 1.2 .3 $F(x)=\left[L^{-1}(x-\sigma(x))\right]^{2}$. 
Por outro lado, dada $F(x)=a_{2} x^{2}+O\left(x^{3}\right)$ com $a_{2}>0$ função analítica em zero tal que $l_{F}=l$, segue do Lema 1.2 .2 que $\sigma(x)=x-\operatorname{sgn}(x) l(G(x))$ é uma involução estrita analítica. Novamente, pelo Lema 1.2.3, $F(x)=\left[L^{-1}(x-\sigma(x))\right]^{2}$.

Podemos dar a seguinte interpretação para os Teoremas 1.3.1 e 1.3.2.

Fixo uma função período analítica $T$, o Teorema 1.3.1 afirma que existem tantos sistemas da forma (1.2) tendo $T$ como função período quantos são os elementos de $\mathfrak{F} \times \Sigma$ onde $\mathfrak{F}$ denota o conjunto das funções analíticas em zero da forma $F(x)=a_{2} x^{2}+O\left(x^{3}\right)$. O Teorema 1.3.2 afirma que existem tantos sistemas analíticos da forma (1.2) com $F \equiv G$ tendo $T$ como função período quantos são os elementos de $\Sigma$.

Teorema 1.3.3 Sejam $l_{F}$ e $l_{G}$ dados por (1.3) associados a $F$ e G respectivamente. Suponhamos que (1.2) seja um sistema $C^{1}$ com centro global na origem e tal que

$$
l_{F}^{\prime}(x) \sim A x^{a} \text { e } l_{G}^{\prime}(x) \sim B x^{b}
$$

em $x=\infty$, isto é, $\lim _{x \rightarrow \infty} \frac{l_{F}^{\prime}(x)-A x^{a}}{x^{a}}=0 e \lim _{x \rightarrow \infty} \frac{l_{G}^{\prime}(x)-B x^{b}}{x^{b}}=0$, com $a>-1, b>-1$, $A>0$ e $B>0$.

Então a função período para o sistema (1.2) satisfaz

$$
T(h) \sim C h^{a+b+1}
$$

em $h=\infty$, isto é, $\lim _{h \rightarrow \infty} \frac{T(h)-C h^{a+b+1}}{h^{a+b+1}}=0$, onde $C=A B \beta(a+1, b+1)$, e $\beta$ é a função Beta.

Para a demonstração desse teorema vamos usar o seguinte Lema.

Lema 1.3.1 Sejam $F$ e $G$ satisfazendo:

(i) $l_{F}^{\prime}(z) \sim A z^{a}$ e $l_{G}^{\prime}(z) \sim B z^{b}$, em $z=\infty$, onde $A>0$ e $B>0$.

(ii) $l_{F}^{\prime}(z) \sim k_{1} z^{-\frac{1}{2}}$ e $l_{G}^{\prime}(z) \sim k_{2} z^{-\frac{1}{2}}$, em $z=0^{+}$, onde $k_{1}>0$ e $k_{2}>0$.

Então

(I) $\exists M>0$ tal que $\left|l_{F}^{\prime}(z)\right| \leq(A+1) z^{a}$ e $\left|l_{G}^{\prime}(z)\right| \leq(B+1) z^{b}$, se $z \geq M$.

(II) $\exists h_{0}>0$ tal que $\left|l_{F}^{\prime}(z)\right| \leq\left(k_{1}+1\right)\left|z^{-\frac{1}{2}}\right|$ e $\left|l_{G}^{\prime}(z)\right| \leq\left(k_{2}+1\right)\left|z^{-\frac{1}{2}}\right|$ se $0<z \leq h_{0}$.

Além disso, existem $C_{F}>0$ e $C_{G}>0$ tais que $\left|l_{F}^{\prime}(z)\right| \leq C_{F} z^{a}$ e $\left|l_{G}^{\prime}(z)\right| \leq C_{G} z^{a}$ se $z \in\left[h_{0}, h-h_{0}\right]$ e $h \geq M+h_{0}$. 
Demonstração. (I) Como $l_{F}^{\prime}(z) \sim A z^{a}$ em $z=\infty$, existe $M_{1}>0$ tal que $\left|l_{F}^{\prime}(z)\right| \leq\left|A z^{a}\right|+\left|l_{F}^{\prime}(z)-A z^{a}\right| \leq A z^{a}+z^{a}=(A+1) z^{a}$ se $z \geq M_{1}$.

Do mesmo modo, como $l_{G}^{\prime}(z) \sim B z^{b}$ em $z=\infty$, existe $M_{2}>0$ tal que $\left|l_{G}^{\prime}(z)\right| \leq\left|B z^{b}\right|+\left|l_{F}^{\prime}(z)-B z^{b}\right| \leq B z^{b}+z^{b}=(B+1) z^{b}$ se $z \geq M_{2}$.

Portanto, basta tomar $M=\max \left\{M_{1}, M_{2}\right\}$. (II) Como $l_{F}^{\prime}(z) \sim k_{1} z^{-\frac{1}{2}}$ em $z=0$, existe $\delta_{1}>0$ tal que $\left|l_{F}^{\prime}(z)\right| \leq\left|k_{1} z^{-\frac{1}{2}}\right|+\left|l_{F}^{\prime}(z)-k_{1} z^{-\frac{1}{2}}\right| \leq k_{1} z^{-\frac{1}{2}}+z^{-\frac{1}{2}}=\left(k_{1}+1\right) z^{-\frac{1}{2}}$ se $0<z \leq \delta_{1}$.

De modo análogo se $l_{G}^{\prime}(z) \sim k_{2} z^{-\frac{1}{2}}$ em $z=0$, existe $\delta_{2}>0$ tal que $\left|l_{G}^{\prime}(z)\right| \leq\left|k_{2} z^{-\frac{1}{2}}\right|+\left|l_{G}^{\prime}(z)-k_{2} z^{-\frac{1}{2}}\right| \leq k_{2} z^{-\frac{1}{2}}+z^{-\frac{1}{2}}=\left(k_{2}+1\right) z^{-\frac{1}{2}}$ se $0<z \leq \delta_{2}$.

Para concluir o que queremos basta tomarmos $h_{0}=\min \left\{\delta_{1}, \delta_{2}\right\}$.

Além disso, $l_{F}^{\prime}(z)$ é contínua em $\left[h_{0}, M\right]$, logo $\left|l_{F}^{\prime}(z)\right|$ possui máximo $N$ em $\left[h_{0}, M\right]$. E mais, $z^{a}$ é contínua e estritamente positiva em $\left[h_{0}, M\right]$, logo possui mínimo $m>0$ nesse intervalo. Desse modo, tomando $A_{1}=\frac{N}{m}$ temos $\left|l_{F}^{\prime}(z)\right| \leq N=A_{1} m \leq A_{1} z^{a}$ se $z \in\left[h_{0}, M\right]$.

Assim, se $C_{F}=\max \left\{A+1, A_{1}\right\}$ e $h \geq M+h_{0}$, decorre usando $(I)$ que para todo $z \in\left[h_{0}, h-h_{0}\right]$ temos $\left|l_{F}^{\prime}(z)\right| \leq C_{F} z^{a}$.

De modo análogo podemos conseguir $C_{G}>0$ tal que se $h \geq M+h_{0}$, então para todo $z \in\left[h_{0}, h-h_{0}\right]$ teremos $\left|l_{G}^{\prime}(z)\right| \leq C_{G} z^{a}$.

Demonstração. (do teorema) Queremos mostrar que

$$
\lim _{h \rightarrow \infty} \frac{T(h)-C h^{a+b+1}}{h^{a+b+1}}=0 .
$$

Como $a>-1$ e $b>-1$ podemos calcular $\beta(a+1, b+1)=\int_{0}^{1} w^{a}(1-w)^{b} d w$ e definir $C=A B \beta(a+1, b+1)=A B \int_{0}^{1} w^{a}(1-w)^{b} d w$.

Usando a Proposição 1.2.2, nossa tese passa a ser

$$
\lim _{h \rightarrow \infty} \frac{\int_{0}^{h} l_{F}^{\prime}(z) l_{G}^{\prime}(h-z) d z-\left(A B \int_{0}^{1} w^{a}(1-w)^{b} d w\right) h^{a+b+1}}{h^{a+b+1}}=0 .
$$

Fazendo a mudança de variáveis $z=h w$ na segunda integral de 1.27 podemos reescrever nossa tese como

$$
\lim _{h \rightarrow \infty} \frac{\int_{0}^{h} l_{F}^{\prime}(z) l_{G}^{\prime}(h-z) d z-\left(A B \int_{0}^{h} \frac{z^{a}}{h^{a}} \frac{(h-z)^{b}}{h^{b}} \frac{d z}{h}\right) h^{a+b+1}}{h^{a+b+1}}
$$


ou ainda como

$$
\lim _{h \rightarrow \infty} \int_{0}^{h} \frac{l_{F}^{\prime}(z) l_{G}^{\prime}(h-z)-A z^{a} B(h-z)^{b}}{h^{a+b+1}} d z=0
$$

Notemos que as funções $l_{F}^{\prime}$ e $l_{G}^{\prime}$ estão definidas em $(0, \infty)$, são contínuas, não negativas e pela observação 1.2 .2 segue que

$$
l_{F}^{\prime}(x) \sim k_{1} x^{-\frac{1}{2}} \text { e } l_{G}^{\prime}(x) \sim k_{2} x^{-\frac{1}{2}} \text { em } x=0
$$

onde $k_{1}>0$ e $k_{2}>0$.

Sejam $M$ e $h_{0}$ dados pelo Lema 1.3 .1 e suponhamos $M>h_{0}$.

Para demonstrar (1.29) vamos dividir o intervalo de integração $[0, h]$, para $h>M+h_{0}$, em três subintervalos, $\left[0, h_{0}\right],\left[h_{0}, h-h_{0}\right]$ e $\left[h-h_{0}, h\right]$, onde $h>h-h_{0} \geq M>h_{0}>0$. Mostraremos que a integral resultante em cada um desses intervalos tende a zero quando $h$ tende a infinito.

Consideraremos inicialmente o primeiro intervalo $\left[0, h_{0}\right]$ e a integral

$$
\int_{0}^{h_{0}} \frac{l_{F}^{\prime}(z) l_{G}^{\prime}(h-z)-A z^{a} B(h-z)^{b}}{h^{a+b+1}} d z .
$$

Nesse caso, $z \in\left[0, h_{0}\right]$ e assim

$$
h-z>h-h_{0}>M>h_{0}>0 .
$$

Então, pelo Lema 1.3.1, teremos $l_{F}^{\prime}(z)=\left|l_{F}^{\prime}(z)\right| \leq\left(k_{1}+1\right) z^{-\frac{1}{2}} \mathrm{e}$ $l_{G}^{\prime}(h-z)=\left|l_{G}^{\prime}(h-z)\right| \leq(B+1)(h-z)^{-\frac{1}{2}}$. Além disso, como $\frac{h-h_{0}}{h} \leq \frac{h-z}{h} \leq 1$ para todo $z \in\left[0, h_{0}\right]$, segue que existe $M_{0} \geq M+h_{0}$ tal que $\left(\frac{h-z}{h}\right)^{b}<2, \forall z \in\left[0, h_{0}\right], \forall h \geq M_{0}$. Logo,

$$
0 \leq \frac{l_{F}^{\prime}(z) l_{G}^{\prime}(h-z)}{h^{a+b+1}} \leq \frac{\left(k_{1}+1\right) z^{-\frac{1}{2}}(B+1)(h-z)^{b}}{h^{a+b+1}} \leq \frac{2\left(k_{1}+1\right)(B+1) z^{-\frac{1}{2}}}{h^{a+1}}
$$

para $z \in\left(0, h_{0}\right]$ e $h \geq M_{0}$.

Observe que $z^{-\frac{1}{2}}$ é uma função integrável em $\left[0, h_{0}\right]$, logo, como $a+1>0$,

$$
\begin{aligned}
0 & \leq \lim _{h \rightarrow \infty} \int_{0}^{h_{0}} \frac{l_{F}^{\prime}(z) l_{G}^{\prime}(h-z)}{h^{a+b+1}} d z \leq \lim _{h \rightarrow \infty} \int_{0}^{h_{0}} 2 \frac{\left(k_{1}+1\right)(B+1) z^{-\frac{1}{2}}}{h^{a+1}} d z \\
& =\lim _{h \rightarrow \infty} \frac{2\left(h_{1}+1\right)(B+1)}{h^{a+1}} \int_{0}^{h_{0}} z^{-\frac{1}{2}} d z=0 .
\end{aligned}
$$


Também, para $h \geq M_{0}$ e $z \in\left(0, h_{0}\right]$ temos

$$
0<\frac{A B z^{a}(h-z)^{b}}{h^{a+b+1}} \leq \frac{2 A B z^{a}}{h^{a+1}} .
$$

Como $a>-1, z^{a}$ é integrável em $\left[0, h_{0}\right]$ e

$$
0 \leq \lim _{h \rightarrow \infty} \int_{0}^{h_{0}} \frac{A z^{a} B(h-z)^{b}}{h^{a+b+1}} d z \leq \lim _{h \rightarrow \infty} \frac{2 A B}{h^{a+1}} \int_{0}^{h_{0}} z^{a} d z=0 .
$$

Portanto vale 1.29 .

Vamos agora passar ao segundo intervalo e provar que

$$
\lim _{h \rightarrow \infty} \int_{h_{0}}^{h-h_{0}} \frac{l_{F}^{\prime}(z) l_{G}^{\prime}(h-z)-A z^{a} B(h-z)^{b}}{h^{a+b+1}} d z=0 .
$$

O integrando nesta integral pode ser escrito como:

$$
\frac{l_{F}^{\prime}(z) l_{G}^{\prime}(h-z)-A z^{a} l_{G}^{\prime}(h-z)}{h^{a+b+1}}+\frac{A z^{a} l_{G}^{\prime}(h-z)-A z^{a} B(h-z)^{b}}{h^{a+b+1}} .
$$

Mostraremos usando o Teorema da Convergência Dominada de Lebesgue (ver [Bar95], [Rud76]) que

$$
\lim _{h \rightarrow \infty} \int_{h_{0}}^{h-h_{0}} \frac{l_{F}^{\prime}(z) l_{G}^{\prime}(h-z)-A z^{a} l_{G}^{\prime}(h-z)}{h^{a+b+1}} d z=0
$$

e

$$
\lim _{h \rightarrow \infty} \int_{h_{0}}^{h-h_{0}} \frac{A z^{a} l_{G}^{\prime}(h-z)-A z^{a} B(h-z)^{b}}{h^{a+b+1}} d z=0
$$

Fazendo a mudança de variáveis $z=h w$ na integral em (1.32) temos:

$$
\begin{aligned}
& \int_{h_{0}}^{h-h_{0}} \frac{l_{F}^{\prime}(z) l_{G}^{\prime}(h-z)-A z^{a} l_{G}^{\prime}(h-z)}{h^{a+b+1}} d z \\
= & \int_{\frac{h_{0}}{h}}^{\frac{h-h_{0}}{h}} \frac{l_{F}^{\prime}(h w) l_{G}^{\prime}(h(1-w))-A(h w)^{a} l_{G}^{\prime}(h(1-w))}{h^{a+b}} d w \\
= & \int_{0}^{1} \frac{l_{F}^{\prime}(h w) l_{G}^{\prime}(h(1-w))-A(h w)^{a} l_{G}^{\prime}(h(1-w))}{h^{a+b}} \chi_{\left[\frac{h_{0}}{h}, \frac{h-h_{0}}{h}\right]}(w) d w \\
= & \int_{0}^{1} \frac{\left[l_{F}^{\prime}(h w)-A(h w)^{a}\right] l_{G}^{\prime}(h(1-w))}{h^{a+b}} \chi_{\left[\frac{h_{0}}{h}, \frac{h-h_{0}}{h}\right]}(w) d w,
\end{aligned}
$$

onde $\chi_{\left[\frac{h_{0}}{h}, \frac{h-h_{0}}{h}\right]}$ é a função característica do conjunto $\left[\frac{h_{0}}{h}, \frac{h-h_{0}}{h}\right]$. 
Para $w \in\left[\frac{h_{0}}{h}, \frac{h-h_{0}}{h}\right]$ temos $h w \in\left[h_{0}, h-h_{0}\right]$ e $h(1-w) \in\left[h_{0}, h-h_{0}\right]$. Então pelo Lema 1.3.1 temos $l_{F}^{\prime}(h w)=\left|l_{F}^{\prime}(h w)\right| \leq C_{F}(h w)^{a} \mathrm{e}$ $l_{G}^{\prime}(h(1-w))=\left|l_{G}^{\prime}(h(1-w))\right| \leq C_{G}(h(1-w))^{b}$. Logo, se $w \in[0,1]$ temos

$$
\begin{aligned}
& \left|\frac{\left[l_{F}^{\prime}(h w)-A(h w)^{a}\right] l_{G}^{\prime}(h(1-w))}{h^{a+b}} \chi_{\left[\frac{h_{0}}{h}, \frac{h-h_{0}}{h}\right]}(w)\right| \\
\leq & \frac{\left[\left|l_{F}^{\prime}(h w)\right|+\left|A(h w)^{a}\right|\right]\left|l_{G}^{\prime}(h(1-w))\right|}{h^{a+b}} \chi_{\left[\frac{h_{0}}{h}, \frac{h-h_{0}}{h}\right]}(w) \\
\leq & \frac{\left[C_{F}(h w)^{a}+A(h w)^{a}\right] C_{G}(h(1-w))^{b}}{h^{a+b}} \chi_{\left[\frac{h_{0}}{h}, \frac{h-h_{0}}{h}\right]}(w) \\
= & \left(C_{F}+A\right) C_{G} w^{a}(1-w)^{b} \chi_{\left[\frac{h_{0}}{h}, \frac{h-h_{0}}{h}\right]}(w) \leq\left(C_{F}+A\right) C_{G} w^{a}(1-w)^{b} .
\end{aligned}
$$

Observe que se $w \in(0,1)$ então

$$
\begin{aligned}
& \lim _{h \rightarrow \infty} \frac{\left[l_{F}^{\prime}(h w)-A(h w)^{a}\right] l_{G}^{\prime}(h(1-w))}{h^{a+b}} \\
= & \lim _{h \rightarrow \infty} \frac{\left(l_{F}^{\prime}(h w)-A(h w)^{a}\right)}{(h w)^{a}} \frac{l_{G}^{\prime}(h(1-w))}{(h(1-w))^{b}} w^{a}(1-w)^{b}=0,
\end{aligned}
$$

pois $\frac{\left(l_{F}^{\prime}(h w)-A(h w)^{a}\right)}{(h w)^{a}} \rightarrow 0$ e $\frac{l_{G}^{\prime}(h(1-w))}{(h(1-w))^{b}} \rightarrow B$ quando $h \rightarrow \infty$.

Já que $w^{a}(1-w)^{b}$ é integrável em $[0,1]$, pois $a>-1$ e $b>-1$, segue de (1.34) e (1.35) que podemos aplicar o Teorema da Convergência Dominada de Lebesgue (ver [Bar95], $[\operatorname{Rud} 76])$ e o limite dado em (1.32) é zero como queríamos.

Fazendo a mesma mudança de variáveis no integrando em (1.33) obtemos:

$$
\lim _{h \rightarrow \infty} \int_{0}^{1} \frac{A(h w)^{a}\left[l_{G}^{\prime}(h(1-w))-B(h(1-w))^{b}\right]}{h^{a+b}} \chi_{\left[\frac{h_{0}}{h}, \frac{h-h_{0}}{h}\right]}(w) d w .
$$

Vamos usar novamente o Lema 1.3 .1 e obter, para $w \in[0,1]$, a majoração

$$
\begin{aligned}
& \left|\frac{A(h w)^{a}\left[l_{G}^{\prime}(h(1-w))-B(h(1-w))^{b}\right]}{h^{a+b}} \chi_{\left[\frac{h_{0}}{h}, \frac{h-h_{0}}{h}\right]}(w)\right| \\
\leq & \frac{A(h w)^{a}\left[C_{G}(h(1-w))^{b}+B(h(1-w))^{b}\right]}{h^{a+b}} \chi_{\left[\frac{h_{0}}{h}, \frac{h-h_{0}}{h}\right]}(w) \\
= & A\left(C_{G}+B\right) w^{a}(1-w)^{b} .
\end{aligned}
$$

Além disso, para $w$ fixo, temos:

$$
\begin{aligned}
& \lim _{h \rightarrow \infty} \frac{A(h w)^{a}\left[l_{G}^{\prime}(h(1-w))-B(h(1-w))^{b}\right]}{h^{a+b}} \\
= & \lim _{h \rightarrow \infty} \frac{A\left[l_{G}^{\prime}(h(1-w))-B(h(1-w))^{b}\right]}{(h(1-w))^{b}}(1-w)^{b} w^{a}=0,
\end{aligned}
$$


pois $\frac{\left[l_{G}^{\prime}(h(1-w))-B(h(1-w))^{b}\right]}{(h(1-w))^{b}} \rightarrow 0$ quando $h \rightarrow \infty$. Assim pelo Teorema da Convergência Dominada de Lebesgue (ver [Bar95], [Rud76]) (1.33) ocorre.

Portanto (1.31) está provado.

Falta ainda provar que:

$$
\lim _{h \rightarrow \infty} \int_{h-h_{0}}^{h} \frac{l_{F}^{\prime}(z) l_{G}^{\prime}(h-z)-A z^{a} B(h-z)^{b}}{h^{a+b+1}} d z=0
$$

Podemos reescrever o integrando acima como segue:

$$
\begin{aligned}
& \frac{l_{F}^{\prime}(z) l_{G}^{\prime}(h-z)-A z^{a} B(h-z)^{b}}{h^{a+b+1}} \\
= & \frac{\left[l_{F}^{\prime}(z)-A z^{a}\right] l_{G}^{\prime}(h-z)}{h^{a+b+1}}+\frac{A z^{a}\left[l_{G}^{\prime}(h-z)-B(h-z)^{b}\right]}{h^{a+b+1}} \\
= & g_{h}(z)+f_{h}(z) .
\end{aligned}
$$

Fazendo $z=h w$ obtemos:

$$
\int_{h-h_{0}}^{h} g_{h}(z) d z=\int_{0}^{1} \frac{\left[l_{F}^{\prime}(h w)-A(h w)^{a}\right] l_{G}^{\prime}(h(1-w))}{h^{a+b}} \chi_{\left[\frac{h-h_{0}}{h}, 1\right]}(w) d w .
$$

Lembremos que $h-h_{0} \geq M$. Para $w \in(0,1) \cap\left[\frac{h-h_{0}}{h}, 1\right]$ temos $h w \in\left(h-h_{0}, h\right)$ e $h(1-w) \in\left(h-h_{0}, h\right)$. Assim pelo Lema 1.3.1,

$$
\begin{aligned}
& \left|\frac{\left[l_{F}^{\prime}(h w)-A(h w)^{a}\right] l_{G}^{\prime}(h(1-w))}{h^{a+b}} \chi_{\left[\frac{h-h_{0}}{h}, 1\right]}(w)\right| \\
\leq & \frac{\left[(A+1)(h w)^{a}+A(h w)^{a}\right](B+1)(h(1-w))^{b}}{h^{a+b}} \chi_{\left[\frac{h-h_{0}}{h}, 1\right]}(w) \\
\leq & (2 A+1)(B+1) w^{a}(1-w)^{b} .
\end{aligned}
$$

Além disso, se $w \in(0,1)$ temos

$$
\frac{\left[l_{F}^{\prime}(h w)-A(h w)^{a}\right] l_{G}^{\prime}(h(1-w))}{h^{a+b}} \rightarrow 0 \text { quando } h \rightarrow \infty .
$$

Portanto pelo Teorema da Convergência Dominada de Lebesgue (ver [Bar95], [Rud76])

$$
\lim _{h \rightarrow \infty} \int_{0}^{1} \frac{\left[l_{F}^{\prime}(h w)-A(h w)^{a}\right] l_{G}^{\prime}(h(1-w))}{h^{a+b}} \chi_{\left[\frac{h-h_{0}}{h}, 1\right]}(w) d w=0 .
$$

Do mesmo modo, para $w \in(0,1) \cap\left[\frac{h-h_{0}}{h}, 1\right]$ temos

$$
\left|\frac{A(h w)^{a}\left[l_{G}^{\prime}(h(1-w))-B(h(1-w))^{b}\right]}{h^{a+b}}\right| \leq A(2 B+1) w^{a}(1-w)^{b} .
$$


Também, se $w \in(0,1)$ então

$$
\frac{A(h w)^{a}\left[l_{G}^{\prime}(h(1-w))-B(h(1-w))^{b}\right]}{h^{a+b}} \rightarrow 0 \text { quando } h \rightarrow \infty .
$$

Novamente pelo Teorema da Convergência Dominada de Lebesgue (ver [Bar95], [Rud76]) obtemos

$$
\lim _{h \rightarrow \infty} \int_{0}^{1} \frac{A(h w)^{a}\left[l_{G}^{\prime}(h(1-w))-B(h(1-w))^{b}\right]}{h^{a+b}} \chi_{\left[\frac{h-h_{0}}{h}, 1\right]}(w) d w=0
$$

\subsection{Sistemas Isócronos}

Esta seção será dedicada ao estudo do problema inverso para encontrar todos os sistemas da forma (1.2) tal que $T$ é uma função constante. Um centro com função período constante é dito um centro isócrono. Assim, dizemos que o sistema (1.2) é isócrono se sua função período for constante.

O Teorema 1.3.1 pode ser usado para caracterizar todos os centros de função período constante do sistema (1.2). Aqui vamos desenvolver alguns casos particulares desse teorema. Para finalizar consideraremos o caso em que (1.2) é polinomial. Lembramos que se $F$ tem a forma simples $F(x)=\alpha x^{2} \operatorname{com} \alpha>0$, então seu comprimento associado é dado por:

$$
l_{F}(h)=F_{+}^{-1}(h)-F_{-}^{-1}(h)=b \sqrt{h} \operatorname{com} b=\frac{2}{\sqrt{\alpha}}>0 .
$$

Por outro lado, da demonstração da Proposição 1.2.1 temos que, se $l(h)=b \sqrt{h}, \operatorname{com} b>0$ então $F_{\sigma}(x)=\frac{(x-\sigma(x))^{2}}{b^{2}}$ tem comprimento associado $l_{F_{\sigma}}(h)=l(h)=b \sqrt{h}$, para cada involução estrita analítica $\sigma$.

Corolário 1.4.1 Suponhamos que a origem de (1.2) é um centro com função período constante e que $l_{F}(h)=b \sqrt{h}$ com $b>0$. Então $l_{G}(h)=a \sqrt{h}$ com $a>0$.

Demonstração. Já que $G$ é analítica, do Lema 1.2.1 sabemos que seu comprimento associado tem a forma

$$
\tilde{l}_{G}(h)=\sqrt{h} \sum_{n=0}^{\infty} a_{n} h^{n}, h \in[0, \epsilon), \text { e } a_{0}>0
$$


Podemos supor sem perda de generalidade que $l_{F}$ esteja definida em $[0, \epsilon)$.

Então

$$
\begin{aligned}
\left(l_{F} * l_{G}\right)(h) & =\int_{0}^{h} l_{F}(h) l_{G}(h-z) d z=\int_{0}^{h} b \sqrt{z} \sqrt{(h-z)} \sum_{n=0}^{\infty} a_{n}(h-z)^{n} d z \\
& =\int_{0}^{h} \sum_{n=0}^{\infty} b a_{n} \sqrt{z(h-z)^{2 n+1}} d z, h \in[0, \epsilon) .
\end{aligned}
$$

Com a mudança de variáveis $z=h \operatorname{sen}^{2} t$

$$
\left(l_{F} * l_{G}\right)(h)=\int_{0}^{h} \sum_{n=0}^{\infty} b a_{n} \sqrt{z(h-z)^{2 n+1}} d z=2 h^{n+2} \int_{0}^{\frac{\pi}{2}}\left(\sum_{n=0}^{\infty} b a_{n} \operatorname{sen}^{2} t \cos ^{2 n+2} t\right) d t .
$$

e usando o Lema 1.2.5 obtemos $\left(l_{F} * l_{G}\right)(h)=\sum_{n=0}^{\infty} b a_{n} I_{1, n+1} h^{n+2}$.

Usando a Proposição 1.2 .2 e lembrando que $T$ é constante temos $a_{n}=0, \forall n \geq 1$.

Logo, $l_{G}(h)=a_{0} \sqrt{h}$ com $a_{0}>0$ como queríamos.

Corolário 1.4.2 Suponha que o centro de (1.2) tem função período constante e que $F=G$. Então o comprimento associado tem a forma $l(h)=a \sqrt{h} \operatorname{com} a>0$.

Demonstração. Do Lema 1.2.1 sabemos que o comprimento associado tem a forma

$$
\begin{aligned}
l_{G}(h) & =l_{F}(h)=l(h)=\sqrt{h} \sum_{n=0}^{\infty} a_{n} h^{n}, h \in[0, \epsilon), a_{0}>0 . \\
\left(l_{F} * l_{G}\right)(h) & =\int_{0}^{h} l_{F}(z) l_{G}(h-z) d z=\int_{0}^{h}\left(\sqrt{z} \sum_{m=0}^{\infty} a_{m} z^{m}\right)\left(\sqrt{(h-z)} \sum_{n=0}^{\infty} a_{n}(h-z)^{n}\right) d z .
\end{aligned}
$$

Fazendo a mudança de variáveis $z=h \operatorname{sen}^{2} t$ obtemos

$$
\begin{aligned}
\int_{0}^{h} z^{\frac{2 m+1}{2}}(h-z)^{\frac{2 n+1}{2}} d z & =\int_{0}^{\frac{\pi}{2}}\left(h \operatorname{sen}^{2} t\right)^{\frac{2 m+1}{2}}\left(h \cos ^{2} t\right)^{\frac{2 n+1}{2}} 2 h \operatorname{sen} t \cos t d t \\
& =2 h^{m+n+2} \int_{0}^{\frac{\pi}{2}}\left(\operatorname{sen}^{2 m+2} t\right)\left(\cos ^{2 n+2} t\right) d t=I_{m+1, n+1} h^{m+n+2} .
\end{aligned}
$$

Portanto, usando o Lema 1.2.5 obtemos

$$
\left(l_{F} * l_{G}\right)(h)=\sum_{m=0}^{\infty} \sum_{n=0}^{\infty} a_{m} a_{n} I_{m+1, n+1} h^{m+n+2} .
$$

Da Proposição 1.2 .2 e do fato de $T$ ser constante vem que $a_{k}=0, \forall k \geq 1$.

Conseqüentemente, $l_{F}(h)=l_{G}(h)=l(h)=a \sqrt{h}$. 
Corolário 1.4.3 Sejam $l_{F}(h)=F_{+}^{-1}(h)-F_{-}^{-1}(h)$ e $l_{G}(h)=G_{+}^{-1}(h)-G_{-}^{-1}(h)$ os comprimentos associados a $F$ e $G$. Suponha que (1.2) é um sistema $C^{1}$ com centro global na origem, de função período constante, e tal que

$$
l_{F}^{\prime}(h) \sim A x^{a} \text { e } l_{G}^{\prime}(h) \sim B x^{b}
$$

em $x=\infty$, com $a>-1, b>-1, A>0$ e $B>0$. Então $a+b+1=0$.

Demonstração. Pelo Teorema 1.3.3, $T(h) \sim C h^{a+b+1}$, isto é,

$$
\lim _{h \rightarrow \infty} \frac{T(h)}{h^{a+b+1}}-C=\lim _{h \rightarrow \infty} \frac{T(h)-C h^{a+b+1}}{h^{a+b+1}}=0 .
$$

Como $T(h)$ é constante diferente de zero e $C \neq 0$ segue que existe $\lim _{h \rightarrow \infty} \frac{1}{h^{a+b+1}}$ e é diferente de zero. Logo $a+b+1=0$.

Corolário 1.4.4 Suponha que $F$ e G são polinômios. Então (1.2) terá função período constante somente se for um sistema linear.

Demonstração. Suponha que a origem de (1.2) é um centro com função período constante. Sabemos que neste caso a fronteira da vizinhança da origem onde só existem órbitas periódicas, não pode ter pontos críticos de (1.2). Este fato faz com que $F$ e $G$ sejam crescentes para $x>0$ e decrescentes para $x<0$. Portanto $F$ e $G$ possuem grau par, digamos $2 n$ e $2 m$, respectivamente, e a origem de (1.2) é um centro global. Portanto

$$
l_{F}^{\prime}(x) \sim A x^{\frac{(1-2 n)}{2 n}} \text { e } l_{G}^{\prime}(x) \sim B x^{\frac{(1-2 m)}{2 m}}
$$

em $x=\infty$, para convenientes constantes positivas $A$ e $B$. Pelo Corolário 1.4.3, devemos ter

$$
\frac{(1-2 n)}{2 n}+\frac{(1-2 m)}{2 m}+1=0, \text { ou seja, } \frac{1}{2 n}+\frac{1}{2 m}-1=0
$$

Este fato faz com que $n=m=1$. Assim, $F$ e $G$ são polinômios de grau 2 e segue a tese.

\subsection{Monotonicidade da função período}

Nesta seção faremos alguns comentários de como usar os resultados anteriores no estudo da monoticidade de $T$ para o sistema (1.2). 
O principal resultado é a próxima Proposição que dá uma expressão para a derivada de $T$ em termos de algumas convoluções.

Sejam $\psi_{F}$ e $\psi_{G}$ definidas por:

$$
\psi_{F}(z)=\frac{l_{F}^{\prime}(z)+2 z l_{F}^{\prime \prime}(z)}{2} \text { e } \psi_{G}(z)=\frac{l_{G}^{\prime}(z)+2 z l_{G}^{\prime \prime}(z)}{2} .
$$

Proposição 1.5.1 Com a notação acima

$$
h T^{\prime}(h)=\left(\psi_{F} * l_{G}^{\prime}\right)(h)+\left(l_{F}^{\prime} * \psi_{G}\right)(h) .
$$

Demonstração. Usando Proposição 1.2 .2 e a mudança de variáveis $z=h \operatorname{sen}^{2} t$ obtemos

$$
\begin{aligned}
T(h) & =\left(l_{F}^{\prime} * l_{G}^{\prime}\right)(h)=\int_{0}^{h} l_{F}^{\prime}(h-z) l_{G}^{\prime}(z) d z \\
& =2 h \int_{0}^{\frac{\pi}{2}} l_{F}^{\prime}\left(h \cos ^{2} t\right) l_{G}^{\prime}\left(h \operatorname{sen}^{2} t\right) \operatorname{sen} t \cos t d t .
\end{aligned}
$$

Assim,

$$
\begin{aligned}
T^{\prime}(h) & =2 \int_{0}^{\frac{\pi}{2}} l_{F}^{\prime}\left(h \cos ^{2} t\right) l_{G}^{\prime}\left(h \operatorname{sen}^{2} t\right) \operatorname{sen} t \cos t d t \\
& +2 h \int_{0}^{\frac{\pi}{2}}\left[l_{F}^{\prime \prime}\left(h \cos ^{2} t\right) l_{G}^{\prime}\left(h \operatorname{sen}^{2} t\right) \operatorname{sen} t \cos ^{3} t+l_{F}^{\prime}\left(h \cos ^{2} t\right) l_{G}^{\prime \prime}\left(h \operatorname{sen}^{2} t\right) \operatorname{sen}^{3} t \cos t\right] d t \\
& =\frac{1}{h}\left(\int_{0}^{h}\left[l_{F}^{\prime}(h-z) l_{G}^{\prime}(z)+l_{F}^{\prime \prime}(h-z) l_{G}^{\prime}(z)(h-z)+l_{F}^{\prime}(h-z) l_{G}^{\prime \prime}(z) z\right] d z\right) .
\end{aligned}
$$

Por outro lado:

$$
\begin{aligned}
& \left(\psi_{F} * l_{G}^{\prime}\right)(h)+\left(l_{F}^{\prime} * \psi_{G}\right)(h) \\
= & \int_{0}^{h}\left[\psi_{F}(h-z) l_{G}^{\prime}(z)+l_{F}^{\prime}(h-z) \psi_{G}(z)\right] d z \\
= & \int_{0}^{h}\left[\frac{l_{F}^{\prime}(h-z)+2(h-z) l_{F}^{\prime \prime}(h-z)}{2} l_{G}^{\prime}(z)+l_{F}^{\prime}(h-z) \frac{l_{G}^{\prime}(z)+2 z l_{G}^{\prime \prime}(z)}{2}\right] d z \\
= & \int_{0}^{h}\left[l_{F}^{\prime}(h-z) l_{G}^{\prime}(z)+l_{F}^{\prime \prime}(h-z) l_{G}^{\prime}(z)(h-z)+l_{F}^{\prime}(h-z) l_{G}^{\prime \prime}(z) z\right] d z
\end{aligned}
$$

De 1.39 e 1.40 segue a tese.

A Proposição 1.5.1 dá algumas condições suficientes para assegurar a monoticidade da função período.

Como $l_{F}^{\prime}$ e $l_{G}^{\prime}$ são funções positivas para todo $h \in(0, \bar{h})$ segue da Proposição 1.5.1 que se $\psi_{F}$ e $\psi_{G}$ são positivas para todo $h \in(0, \bar{h})$ então $T$ é monótona crescente, pois 
convolução de funções positivas é positiva. Do mesmo modo se $\psi_{F}$ e $\psi_{G}$ são funções negativas para todo $h \in(0, \bar{h})$ então $T$ é decrescente.

Vamos agora desenvolver outra expressão para $\psi_{F}$.

Para $h \in[0, \bar{h})$, sejam $x_{h+}=F_{+}^{-1}(h)$ e $x_{h-}=F_{-}^{-1}(h)$.

Como $F_{+}^{-1}$ é a inversa de $\left.F\right|_{\left[0, \bar{x}_{+}\right)}:\left[0, \bar{x}_{+}\right) \rightarrow[0, \bar{h})$ decorre que

$$
\left(F_{+}^{-1}\right)^{\prime}(h)=\frac{1}{F^{\prime}\left(F_{+}^{-1}(h)\right)}=\frac{1}{F^{\prime}\left(x_{h+}\right)}
$$

Derivando (1.41) em relação a h, temos:

$$
\left(F_{+}^{-1}\right)^{\prime \prime}(h)=-\frac{F^{\prime \prime}\left(F_{+}^{-1}(h)\right)}{\left[F^{\prime}\left(F_{+}^{-1}(h)\right)\right]^{2}} \frac{1}{F^{\prime}\left(F_{+}^{-1}(h)\right)}=\frac{-F^{\prime \prime}\left(x_{h+}\right)}{\left(F^{\prime}\left(x_{h+}\right)\right)^{3}}
$$

Analogamente, como $F_{-}^{-1}$ é a inversa de $\left.F\right|_{\left(\bar{x}_{-}, 0\right]}:\left(\bar{x}_{-}, 0\right] \rightarrow[0, \bar{h})$ obtemos

$$
\left(F_{-}^{-1}\right)^{\prime}(h)=\frac{1}{F^{\prime}\left(F_{-}^{-1}(h)\right)}=\frac{1}{F^{\prime}\left(x_{h-}\right)}
$$

e

$$
\left(F_{-}^{-1}\right)^{\prime \prime}(h)=-\frac{F^{\prime \prime}\left(F_{-}^{-1}(h)\right)}{\left[F^{\prime}\left(F_{-}^{-1}(h)\right)\right]^{3}}=\frac{-F^{\prime \prime}\left(x_{h-}\right)}{\left(F^{\prime}\left(x_{h-}\right)\right)^{3}}
$$

Usando as igualdades (1.41), (1.42), (1.43), (1.44), podemos dar uma outra expressão para $\psi_{F}$ em função de $F$, a saber:

$$
\begin{aligned}
\psi_{F}(h) & =\frac{l_{F}^{\prime}(h)+2 h l_{F}^{\prime \prime}(h)}{2} \\
& =\frac{\left(F_{+}^{-1}\right)^{\prime}(h)-\left(F_{-}^{-1}\right)^{\prime}(h)+2 h\left[\left(F_{+}^{-1}\right)^{\prime \prime}(h)-\left(F_{-}^{-1}\right)^{\prime \prime}(h)\right]}{2} \\
& =\frac{1}{2}\left[\left(F_{+}^{-1}\right)^{\prime}(h)+2 h\left(F_{+}^{-1}\right)^{\prime \prime}(h)-\left(F_{-}^{-1}\right)^{\prime}(h)-2 h\left(F_{-}^{-1}\right)^{\prime \prime}(h)\right] \\
& =\frac{1}{2}\left[\frac{1}{F^{\prime}\left(x_{h+}\right)}+2 F\left(x_{h+}\right)\left(\frac{-F^{\prime \prime}\left(x_{h+}\right)}{\left(F^{\prime}\left(x_{h+}\right)\right)^{3}}\right)-\frac{1}{F^{\prime}\left(x_{h-}\right)}-2 F\left(x_{h-}\right)\left(\frac{-F^{\prime \prime}\left(x_{h-}\right)}{\left(F^{\prime}\left(x_{h-}\right)\right)^{3}}\right)\right] \\
& =\frac{1}{2}\left[\frac{\left(F^{\prime}\left(x_{h+}\right)\right)^{2}-2 F\left(x_{h+}\right) F^{\prime \prime}\left(x_{h+}\right)}{\left(F^{\prime}\left(x_{h+}\right)\right)^{3}}-\frac{\left(F^{\prime}\left(x_{h-}\right)\right)^{2}-2 F\left(x_{h-}\right) F^{\prime \prime}\left(x_{h-}\right)}{\left(F^{\prime}\left(x_{h-}\right)\right)^{3}}\right]
\end{aligned}
$$

Analisando (1.45) vemos que um caminho para assegurar que $\psi_{F}$ seja positivo é exigir que a função $\left(F^{\prime}(x)\right)^{2}-2 F(x) F^{\prime \prime}(x)$ seja positiva, pois $F\left(x_{h+}\right)>0, F\left(x_{h-}\right)>0$, $F^{\prime}\left(x_{h+}\right)>0$ e $F^{\prime}\left(x_{h-}\right)<0$ para $h \in(0, \bar{h})$. 
Seja $\phi_{F}$ definida por

$$
\phi_{F}(x)=\frac{\left(F^{\prime}(x)\right)^{2}-2 F(x) F^{\prime \prime}(x)}{\left(F^{\prime}(x)\right)^{3}}=\left(\frac{F(x)}{\left(F^{\prime}(x)\right)^{2}}\right)^{\prime} .
$$

Então $\psi_{F}(h)=\frac{1}{2}\left(\phi_{F}\left(x_{h+}\right)-\phi_{F}\left(x_{h-}\right)\right)$.

Podemos ver que existe $\lim _{x \rightarrow 0} \phi_{F}(x)$ supondo que $F(x)=a_{2} x^{2}+O\left(x^{3}\right)$ com $a_{2}>0$.

Portanto, se $\phi_{F}$ é estritamente crescente então $\psi_{F}$ será sempre positiva. Isto nos dá uma condição fraca para a monotonicidade de $T$.

Os resultados apresentados e demonstrados neste capítulo são essencialmente aqueles do artigo [CGM00], que estudamos e de onde tiramos as idéias para escrever os próximos dois capítulos, que contém resultados e demonstrações que generalizam, num certo sentido, estes apresentados aqui.

Nossa contribuição neste primeiro capítulo foi apenas no sentido de detalhar um pouco mais as demonstrações presentes em [CGM00], procurando tornar a apresentação mais didática. 


\section{Generalização dos resultados}

\section{anteriores.}

Neste capítulo vamos procurar demonstrar resultados análogos aos do capítulo anterior, iniciando com a busca de um caminho que permita tratar o caso de funções analíticas $G$ da forma $G(y)=b y^{2 N}+O\left(y^{2 N+1}\right), N \in \mathbb{N}^{*}$.

Quando um sistema hamiltoniano tem energia cinética $F(x)=a x^{2}+O\left(x^{3}\right), a>0$, e energia potencial $G(y)=b y^{2 N}+O\left(y^{2 N+1}\right), b>0$, onde $N>1$ mesmo que estas sejam analíticas a função período do centro na origem não será analítica em zero, pois o período tenderá a infinito quando as condições iniciais se aproximam do ponto de equilíbrio.

Na seção 2.1 obteremos a forma da função período no caso em que $G(y)=b y^{4}+$ $O\left(y^{5}\right)$ e no caso em que $G(y)=b y^{6}+O\left(y^{7}\right)$. Motivados pelos resultados da seção 2.1 generalizaremos os resultados de [CGM00]. Isto será apresentado na seção 2.2.

Em todo o capítulo, a menos de menção explícita em contrário, $\Omega$ e $\widetilde{\Omega}$ denotarão respectivamente os domínios de analiticidade de $F$ e $G$, que terão sempre mínimo estrito na origem. Como no capítulo 1 , usaremos $\bar{x}_{-}<0<\bar{x}_{+}, \bar{y}_{-}<0<\bar{y}_{+}$e $\bar{h}>0$ tais que

(i) $\left[\bar{x}_{-}, \bar{x}_{+}\right] \subset \Omega$,

(ii) $\left[\bar{y}_{-}, \bar{y}_{+}\right] \subset \widetilde{\Omega}$,

(iii) $F$ é estritamente decrescente em $\left[\bar{x}_{-}, 0\right)$ e estritamente crescente em $\left(0, \bar{x}_{+}\right]$, e

$F^{\prime}(x) \neq 0$ para $x$ nesses intervalos,

(iv) $G$ é estritamente decrescente em $\left[\bar{y}_{-}, 0\right)$ e estritamente crescente em $\left(0, \bar{y}_{+}\right]$, e

$G^{\prime}(y) \neq 0$ para $y$ nesses intervalos,

(v) $\bar{h}=F\left(\bar{x}_{-}\right)=F\left(\bar{x}_{+}\right)=G\left(\bar{y}_{-}\right)=G\left(\bar{y}_{+}\right)$. 


\subsection{A função período em dois exemplos ilustrativos.}

Nesta seção procuraremos uma conveniente classe de funções onde estarão as funções período para sistemas hamiltonianos com energia cinética analítica em zero $F(x)=a x^{2}+O\left(x^{3}\right), a>0$, e energia potencial analítica em zero $G(y)=b y^{2 N}+O\left(y^{2 N+1}\right)$, $b>0$, no caso em que $N=2$ ou $N=3$.

Observação 2.1.1 Se $\widetilde{\Omega}$ é uma vizinhança da origem e $G(y)=b y^{4}+O\left(y^{5}\right) \operatorname{com} b>0$ é analítica em $\widetilde{\Omega}$, então $K$ definida por $K(y)=\frac{G(y)}{y^{4}}$ se $y \neq 0$ e $K(0)=b$, é também analítica em $\widetilde{\Omega}$ pois $K$ é analítica em $\widetilde{\Omega}-\{0\}$ e para y numa vizinhança de zero, $G(y)=\sum_{n=4}^{\infty} b_{n} y^{n}$ e portanto $K(y)=\sum_{n=0}^{\infty} b_{n+4} y^{n}$. Além disso, para $y \in\left[\bar{y}_{-}, \bar{y}_{+}\right]$temos $K(y)>0$. Concluímos que $\psi(y)=\sqrt[4]{K(y)}$ é analítica em $\left(\bar{y}_{-}, \bar{y}_{+}\right)$.

Fato 2.1.1 Seja $G(y)=b y^{4}+O\left(y^{5}\right)$ com $b>0$, analítica em $\widetilde{\Omega}$. Para $h \in(0, \bar{h})$ considere a função $l_{G}$ dada por $l_{G}(h)=G_{+}^{-1}(h)-G_{-}^{-1}(h)$. Então

$$
l_{G}(h)=\sqrt[4]{h}\left(\sum_{n=0}^{\infty} b_{4 n+1} h^{n}+\sqrt{h} \sum_{n=0}^{\infty} b_{4 n+3} h^{n}\right), \quad \text { com } b_{1}=\frac{2}{\sqrt[4]{b}}>0 .
$$

Demonstração. Sejam $K$ e $\psi$ as funções definidas na observação 2.1.1. Considere a função $\phi:\left(\bar{y}_{-}, \bar{y}_{+}\right) \rightarrow(-\sqrt[4]{\bar{h}}, \sqrt[4]{\bar{h}})$, definida por

$$
\phi(y)=\operatorname{sgn}(y) \sqrt[4]{G(y)} .
$$

Essa função é inversível e já que $\phi(0)=0$ e $\phi(y)=\frac{y}{|y|} \sqrt[4]{y^{4} K(y)}=y \sqrt[4]{K(y)}=y \psi(y)$, em $\left(\bar{y}_{-}, \bar{y}_{+}\right)-\{0\}$, resulta que $\phi$ é analítica em $\left(\bar{y}_{-}, \bar{y}_{+}\right)$e que $\phi^{\prime}(0)=\sqrt[4]{b}>0$. Também $\phi^{\prime}(y)=\frac{G^{\prime}(y)}{4 \sqrt[4]{(G(y))^{3}}}>0$ em $\left(0, \bar{y}_{+}\right)$e $\phi^{\prime}(y)=\frac{-G^{\prime}(y)}{4 \sqrt[4]{(G(y))^{3}}}>0$ em $\left(\bar{y}_{-}, 0\right)$. Logo $\phi^{\prime}(y)>0$ em $\left(\bar{y}_{-}, \bar{y}_{+}\right)$. Assim, $\phi$ é inversível e sua inversa é analítica em $(-\sqrt[4]{\bar{h}}, \sqrt[4]{\bar{h}})$.

Se $y_{+} \in\left(0, \bar{y}_{+}\right)$e $h \in(0, \bar{h})$ são tais que $G_{+}\left(y_{+}\right)=G\left(y_{+}\right)=h$ então $\phi\left(y_{+}\right)=\sqrt[4]{h}$ e portanto $G_{+}^{-1}(h)=y_{+}=\phi^{-1}(\sqrt[4]{h})$. Analogamente $G_{-}^{-1}(h)=y_{-}=\phi^{-1}(-\sqrt[4]{h}) \operatorname{Logo}$ podemos escrever $l_{G}$ como

$$
l_{G}(h)=\phi^{-1}(\sqrt[4]{h})-\phi^{-1}(-\sqrt[4]{h})
$$

Além disso a função

$$
I(h)=\phi^{-1}(h)-\phi^{-1}(-h)
$$


é ímpar e analítica em $(-\sqrt[4]{\bar{h}}, \sqrt[4]{\bar{h}})$. Para $s$ suficientemente pequeno, temos:

$$
\left.I(s)=\phi^{-1}(s)-\phi^{-1}(-s)\right)=\sum_{n=0}^{\infty} b_{2 n+1} s^{2 n+1}
$$

onde $b_{1}=I^{\prime}(0)=\frac{2}{\sqrt[4]{b}}$. Portanto, para $h>0$ suficiente pequeno temos

$$
l_{G}(h)=\sum_{n=0}^{\infty} b_{2 n+1}(\sqrt[4]{h})^{2 n+1}=\sqrt[4]{h} \sum_{n=0}^{\infty} b_{2 n+1}(h)^{\frac{n}{2}}=\sqrt[4]{h}\left(\sum_{n=0}^{\infty} b_{4 n+1} h^{n}+\sqrt{h} \sum_{n=0}^{\infty} b_{4 n+3} h^{n}\right)
$$

como queríamos.

Fato 2.1.2 Sejam $F(x)=a x^{2}+O\left(x^{3}\right)$ analítica em $\Omega$ com $a>0$ e $G(y)=b y^{4}+O\left(y^{5}\right)$ analítica em $\widetilde{\Omega}$ com $b>0$. Então valem as seguintes afirmações:

(a) $l_{F}^{\prime}(h)=\frac{A(h)}{\sqrt{h}}$ com $A$ analítica em zero e $A(0)>0$.

(b) $l_{G}^{\prime}(h)=\frac{B_{1}(h)}{\sqrt[4]{h^{3}}}+\frac{B_{2}(h)}{\sqrt[4]{h}}$ com $B_{1}$ e $B_{2}$ analíticas em zero e $B_{1}(0)>0$.

(c) Existem $T_{1}$ e $T_{2}$ analíticas em zero com $T_{1}(0)>0$ tais que

$$
T(h)=\frac{1}{\sqrt[4]{h}}\left(T_{1}(h)+\sqrt{h} T_{2}(h)\right) .
$$

Demonstração. (a) Decorre do Lema 1.2.1.

(b) Pelo Fato 2.1.1, $l_{G}(h)=\sqrt[4]{h}\left(\mathcal{G}_{1}(h)+\sqrt{h} \mathcal{G}_{2}(h)\right)$, com $\mathcal{G}_{1}$ e $\mathcal{G}_{2}$ analíticas numa vizinhança de zero e $\mathcal{G}_{1}(0)=\frac{2}{\sqrt[4]{b}}>0$, então

$$
\begin{aligned}
l_{G}^{\prime}(h) & =\frac{1}{4 \sqrt[4]{h^{3}}}\left(\mathcal{G}_{1}(h)+\sqrt{h} \mathcal{G}_{2}(h)\right)+\sqrt[4]{h}\left(\mathcal{G}_{1}^{\prime}(h)+\frac{1}{2 \sqrt{h}} \mathcal{G}_{2}(h)+\sqrt{h} \mathcal{G}_{2}^{\prime}(h)\right) \\
& =\frac{\mathcal{G}_{1}(h)+4 h \mathcal{G}_{1}^{\prime}(h)}{4 \sqrt[4]{h^{3}}}+\frac{3 \mathcal{G}_{2}(h)+4 h \mathcal{G}_{2}^{\prime}(h)}{4 \sqrt[4]{h}} \\
& =\frac{B_{1}(h)}{\sqrt[4]{h^{3}}}+\frac{B_{2}(h)}{\sqrt[4]{h}},
\end{aligned}
$$

onde $B_{1}(h)=\frac{\mathcal{G}_{1}(h)+4 h \mathcal{G}_{1}^{\prime}(h)}{4}, B_{2}(h)=\frac{3 \mathcal{G}_{2}(h)+4 h \mathcal{G}_{2}^{\prime}(h)}{4}$ são funções analíticas em zero, $\operatorname{com} B_{1}(0)=\frac{\mathcal{G}_{1}(0)}{4}=\frac{2}{4 \sqrt[4]{b}}>0$.

(c) Pela Proposição 1.2.2, temos:

$$
\begin{aligned}
T(h) & =\left(l_{F}^{\prime} * l_{G}^{\prime}\right)(h)=\int_{0}^{h} l_{F}^{\prime}(h-z) l_{G}^{\prime}(z) d z \\
& =\int_{0}^{h}\left(\frac{B_{1}(z)}{\sqrt[4]{(z)^{3}}}+\frac{B_{2}(z)}{\sqrt[4]{z}}\right) \frac{A(h-z)}{\sqrt{h-z}} d z .
\end{aligned}
$$


Sejam $B_{1}(h)=\sum_{n=0}^{\infty} b_{n}^{1} h^{n}, B_{2}(h)=\sum_{n=0}^{\infty} b_{n}^{2} h^{n}$ e $A(h)=\sum_{n=0}^{\infty} a_{n} h^{n}$ as expansões em série de Taylor de $B_{1}, B_{2}$ e $A$ em uma vizinhança de zero.

Façamos em (2.2) a mudança de variável $z=h \cos ^{2} \theta$.

$$
\begin{aligned}
T(h) & =\frac{2}{\sqrt[4]{h}}\left[\int_{0}^{\frac{\pi}{2}} B_{1}\left(h \cos ^{2} \theta\right) \frac{A\left(h \operatorname{sen}^{2} \theta\right)}{\sqrt{\cos \theta}} d \theta+\sqrt{h} \int_{0}^{\frac{\pi}{2}} \sqrt{\cos \theta} B_{2}\left(h \cos ^{2} \theta\right) A\left(h \operatorname{sen}^{2} \theta\right) d \theta\right] \\
T(h) & =\frac{2}{\sqrt[4]{h}}\left[\int_{0}^{\frac{\pi}{2}}\left(\sum_{j=0}^{\infty} b_{j}^{1}\left(h \cos ^{2} \theta\right)^{j}\right)\left(\sum_{i=0}^{\infty} \frac{a_{i}\left(h \operatorname{sen}^{2} \theta\right)^{i}}{\sqrt{\cos \theta}}\right) d \theta\right. \\
& \left.+\sqrt{h} \int_{0}^{\frac{\pi}{2}} \sqrt{\cos \theta}\left(\sum_{j=0}^{\infty} b_{j}^{2}\left(h \cos ^{2} \theta\right)^{j}\right)\left(\sum_{i=0}^{\infty} a_{i}\left(h \operatorname{sen}^{2} \theta\right)^{i}\right) d \theta\right]
\end{aligned}
$$

Pelo Lema 1.2.5 segue que

$$
\begin{aligned}
T(h) & =\frac{2}{\sqrt[4]{h}}\left[\int_{0}^{\frac{\pi}{2}} \sum_{n=0}^{\infty}\left(\sum_{i+j=n} \frac{\left(b_{j}^{1} a_{i} \cos ^{2 j} \theta \operatorname{sen}^{2 i} \theta\right)}{\sqrt{\cos \theta}}\right) h^{n} d \theta\right. \\
& \left.+\sqrt{h} \int_{0}^{\frac{\pi}{2}} \sum_{n=0}^{\infty}\left(\sum_{i+j=n}\left(b_{j}^{2} a_{i} \cos ^{2 j} \theta \operatorname{sen}^{2 i} \theta \sqrt{\cos \theta}\right)\right) h^{n} d \theta\right] \\
& =\frac{1}{\sqrt[4]{h}}\left[\sum_{n=0}^{\infty}\left(\sum_{i+j=n} b_{j}^{1} a_{i}\left(2 \int_{0}^{\frac{\pi}{2}} \cos ^{\left(2 j-\frac{1}{2}\right)} \theta \operatorname{sen}^{2 i} \theta d \theta\right)\right) h^{n}\right. \\
& \left.+\sqrt{h} \sum_{n=0}^{\infty}\left(\sum_{i+j=n} b_{j}^{2} a_{i}\left(2 \int_{0}^{\frac{\pi}{2}} \cos ^{\left(2 j+\frac{1}{2}\right)} \theta \operatorname{sen}^{2 i} \theta d \theta\right)\right) h^{n}\right] \\
& =\frac{1}{\sqrt[4]{h}}\left[\sum_{n=0}^{\infty}\left(\sum_{i+j=n} b_{j}^{1} a_{i} I_{i, j}^{1}\right) h^{n}+\sqrt{h} \sum_{n=0}^{\infty}\left(\sum_{i+j=n} b_{j}^{2} a_{i} I_{i, j}^{2}\right) h^{n}\right]
\end{aligned}
$$

onde

$$
I_{i, j}^{1}=2 \int_{0}^{\frac{\pi}{2}} \cos ^{\left(2 j-\frac{1}{2}\right)} \theta \operatorname{sen}^{2 i} \theta d \theta \text { e } I_{i, j}^{2}=2 \int_{0}^{\frac{\pi}{2}} \cos ^{\left(2 j+\frac{1}{2}\right)} \theta \operatorname{sen}^{2 i} \theta d \theta,
$$

e que $T_{1}(h)=\sum_{n=0}^{\infty} t_{n}^{1} h^{n}$ e $T_{2}(h)=\sum_{n=0}^{\infty} t_{n}^{2} h^{n}$ convergem numa vizinhança de zero onde

$$
t_{n}^{1}=\sum_{i+j=n} b_{j}^{1} a_{i} I_{i, j}^{1} \text { e } t_{n}^{2}=\sum_{i+j=n} b_{j}^{2} a_{i} I_{i, j}^{2}, n \in \mathbb{N}
$$

Assim, $T$ é dada por $T(h)=\frac{1}{\sqrt[4]{h}}\left(T_{1}(h)+\sqrt{h} T_{2}(h)\right)$ e $T_{1}(0)=t_{0}^{1}=b_{0}^{1} a_{0} I_{0,0}^{1}=B_{1}(0) A(0) I_{0,0}^{1}>0$. 
Fato 2.1.3 Seja $G(y)=b y^{6}+O\left(y^{7}\right)$ analítica em $\widetilde{\Omega}$ com $b>0$. Para $h \in(0, \bar{h})$ considere a função $l_{G}$ dada por $l_{G}=G_{+}^{-1}(h)-G_{-}^{-1}(h)$. Então

$$
l_{G}(h)=\sqrt[6]{h}\left(\sum_{n=0}^{\infty} b_{6 n+1} h^{n}+\sqrt[3]{h} \sum_{n=0}^{\infty} b_{6 n+3} h^{n}+\sqrt[3]{h^{2}} \sum_{n=0}^{\infty} b_{6 n+5} h^{n}\right), \quad \text { onde } b_{1}=\frac{2}{\sqrt[6]{b}}>0 .
$$

Demonstração. Análoga à prova do Fato 2.1.1.

Observação 2.1.2 Usando o Fato 2.1.3 podemos, como no ítem (b) do Fato 2.1.2, mostrar que

$$
l_{G}^{\prime}(h)=\frac{B_{1}(h)}{\sqrt[6]{h^{5}}}+\frac{B_{2}(h)}{\sqrt[6]{h^{3}}}+\frac{B_{3}(h)}{\sqrt[6]{h}}
$$

onde $B_{1}, B_{2}$ e $B_{3}$ são funções analíticas em zero com $B_{1}(0)=\frac{b_{1}}{6}>0$.

Fato 2.1.4 Sejam $F(x)=a x^{2}+O\left(x^{3}\right)$ analítica em $\Omega$ com $a>0$ e $G(y)=b y^{6}+O\left(y^{7}\right)$ analítica em $\tilde{\Omega}$ com $b>0$. Então a função período para (1.2) tem a forma

$$
T(h)=\frac{1}{\sqrt[3]{h}}\left[T_{1}(h)+\sqrt[3]{h} T_{2}(h)+\sqrt[3]{h^{2}} T_{3}(h)\right]
$$

onde $T_{1}, T_{2}$ e $T_{3}$ são analíticas em zero e $T_{1}(0)>0$.

Demonstração. Usando o Fato 2.1.3 e a Observação 2.1.2, a prova fica análoga ao ítem (c) do Fato 2.1.2.

Observação 2.1.3 Seja $F$ analítica da forma $F(x)=a x^{2}+O\left(x^{3}\right), a>0$. Pelos resultados anteriores vemos que:

$G(y)=b y^{2}+O\left(y^{3}\right), b>0 \Rightarrow l_{G}^{\prime}(h)=\frac{B(h)}{\sqrt{h}}$, com $B$ analítica em zero e $B(0)>0$, e $T$ é analítica em zero com $T(0)>0$.

$G(y)=b y^{4}+O\left(y^{5}\right), b>0 \Rightarrow l_{G}^{\prime}(h)=\frac{B_{1}(h)}{\sqrt[4]{h^{3}}}+\frac{B_{2}(h)}{\sqrt[4]{h}}$ com $B_{1}$ e $B_{2}$ analíticas em zero, $B_{1}(0)>0$, e $T(h)=\frac{1}{\sqrt[4]{h}}\left(T_{1}(h)+\sqrt{h} T_{2}(h)\right)$ com $T_{1}, T_{2}$ analíticas em zero e $T_{1}(0)>0$.

$G(y)=b y^{6}+O\left(y^{7}\right), b>0 \Rightarrow l_{G}^{\prime}(h)=\frac{B_{1}(h)}{\sqrt[6]{h^{5}}}+\frac{B_{2}(h)}{\sqrt[6]{h^{3}}}+\frac{B_{3}(h)}{\sqrt[6]{h}}$ com $B_{1}, B_{2}$ e $B_{3}$ analíticas em zero, $B_{1}(0)>0$, e $T(h)=\frac{1}{\sqrt[3]{h}}\left(T_{1}(h)+\sqrt[3]{h} T_{2}(h)+\sqrt[3]{h^{2}} T_{3}(h)\right)$ com $T_{1}, T_{2}$ e $T_{3}$ analíticas em zero e $T_{1}(0)>0$. 


\subsection{Generalização do Capítulo 1}

Nesta seção está nosso resultado principal, Teorema 2.2.1. Nela estenderemos os resultados de [CGM00] (ver capítulo 1 deste trabalho) para sistemas hamiltonianos com energia cinética analítica em zero $F(x)=a x^{2}+O\left(x^{3}\right), a>0$, e energia potencial $G(y)=b y^{2 N}+O\left(y^{2 N+1}\right), b>0$, ambas analíticas em zero.

\subsubsection{Resultados preliminares}

Apresentaremos aqui resultados análogos aos da seção 1.2 do capítulo 1, que serão usados em nossos resultados principais.

Observação 2.2.1 Se $G(y)=b y^{2 N}+O\left(y^{2 N+1}\right)$ com $b>0$ é analítica em $\widetilde{\Omega}$, então $K$ definida por $K(y)=\frac{G(y)}{y^{2 N}}$, se $y \neq 0$, e $K(0)=b$, é também analítica em $\widetilde{\Omega}$, pois $K$ é analítica em $\widetilde{\Omega}-\{0\}$ e, numa vizinhança de zero, $G(y)=\sum_{n=0}^{\infty} a_{2 N+n} y^{2 N+n}$ e portanto $K(y)=\sum_{n=0}^{\infty} a_{2 N+n} y^{n}$. Além disso, para $y \in\left[\bar{y}_{-}, \bar{y}_{+}\right]$temos $K(y)>0$. Concluímos que $\psi(y)=\sqrt[2 N]{K(y)}$ é analítica em $\left(\bar{y}_{-}, \bar{y}_{+}\right)$.

O nosso próximo resultado procura estender o Lema 1.2.1 e os Fatos 2.1.1 e 2.1.3 para $G(y)=b y^{2 N}+O\left(y^{2 N+1}\right)$ analítica em zero com $b>0$ e $N \in \mathbb{N}^{*}$.

Lema 2.2.1 Seja $G(y)=b y^{2 N}+O\left(y^{2 N+1}\right) \operatorname{com} b>0$, analítica em $\widetilde{\Omega}$. Para $h \in(0, \bar{h})$ considere a função $l_{G}$ dada por $l_{G}(h)=G_{+}^{-1}(h)-G_{-}^{-1}(h)$. Então

$$
l_{G}(h)=\sqrt[2 N]{h}\left(\mathcal{G}_{1}(h)+\sqrt[N]{h} \mathcal{G}_{2}(h)+\cdots+\sqrt[N]{h^{N-1}} \mathcal{G}_{N}(h)\right)
$$

onde $\mathcal{G}_{1}, \mathcal{G}_{2}, \cdots, \mathcal{G}_{N}$ são funções analíticas e $\mathcal{G}_{1}(0)=\frac{2}{\sqrt[2 N]{b}}>0$.

Demonstração. Sejam $K$ e $\psi$ as funções definidas na observação 2.2.1. Considere a função $\phi:\left(\bar{y}_{-}, \bar{y}_{+}\right) \rightarrow(-\sqrt[2 N]{\bar{h}}, \sqrt[2 N]{\bar{h}})$, definida por

$$
\phi(y)=\operatorname{sgn}(y) \sqrt[2 N]{G(y)}
$$

Essa função é inversível e já que

$$
\phi(0)=0 \text { e } \phi(y)=\frac{y}{|y|} \sqrt[2 N]{y^{2 N} K(y)}=y \sqrt[2 N]{K(y)}=y \psi(y) \text { em }\left(\bar{y}_{-}, \bar{y}_{+}\right)-\{0\},
$$


resulta que $\phi$ é analítica em $\left(\bar{y}_{-}, \bar{y}_{+}\right)\left[\right.$Rud76] e que $\phi^{\prime}(0)=\sqrt[2 N]{b}>0$. Também $\phi^{\prime}(y)=\frac{G^{\prime}(y)}{2 N \sqrt[2 N]{(G(y))^{2 N-1}}}>0 \mathrm{em}\left(0, \bar{y}_{+}\right)$e $\phi^{\prime}(y)=\frac{-G^{\prime}(y)}{2 N \sqrt[2 N]{(G(y))^{2 N-1}}}>0 \mathrm{em}\left(\bar{y}_{-}, 0\right)$. Logo $\phi^{\prime}(y)>0$ em $\left(\bar{y}_{-}, \bar{y}_{+}\right)$. Assim, $\phi$ é inversível e sua inversa é analítica em $(-\sqrt[2 N]{\bar{h}}, \sqrt[2 N]{\bar{h}})$.

Se $y_{+} \in\left(0, \bar{y}_{+}\right)$e $h \in(0, \bar{h})$ são tais que $G_{+}\left(y_{+}\right)=G\left(y_{+}\right)=h$ então $\phi\left(y_{+}\right)=\sqrt[2 N]{h}$ e portanto $G_{+}^{-1}(h)=y_{+}=\phi^{-1}(\sqrt[2 N]{h})$. Analogamente $G_{-}^{-1}(h)=\phi^{-1}(-\sqrt[2 N]{h})$. Assim podemos escrever $l_{G}$ como

$$
l_{G}(h)=\phi^{-1}(\sqrt[2 N]{h})-\phi^{-1}(-\sqrt[2 N]{h})
$$

A função $I(h)=\phi^{-1}(h)-\phi^{-1}(-h)$ é ímpar, assim podemos escrever $I(h)=\sum_{n=0}^{\infty} a_{2 n+1} h^{2 n+1}$
e

$$
\begin{aligned}
l_{G}(h) & =\sum_{n=0}^{\infty} a_{2 n+1}(\sqrt[2 N]{h})^{2 n+1}=\sqrt[2 N]{h} \sum_{n=0}^{\infty} a_{2 n+1} h^{\frac{n}{N}} \\
& =\sqrt[2 N]{h}\left[\sum_{n=0}^{\infty} a_{2 N n+1} h^{n}+\sqrt[N]{h} \sum_{n=0}^{\infty} a_{2 N n+3} h^{n}+\cdots+\sqrt[N]{h^{N-1}} \sum_{n=0}^{\infty} a_{2 N n+(2 N-1)} h^{n}\right] \\
& =\sqrt[2 N]{h}\left[\mathcal{G}_{1}(h)+\sqrt[N]{h} \mathcal{G}_{2}(h)+\cdots+\sqrt[N]{h^{N-1}} \mathcal{G}_{N}(h)\right]
\end{aligned}
$$

onde $\mathcal{G}_{1}, \mathcal{G}_{2}, \cdots, \mathcal{G}_{N}$ são analíticas em zero. Além disso, $\mathcal{G}_{1}(0)=a_{1}=I^{\prime}(0)=\frac{2}{\sqrt[2 N]{b}}>0$.

Lema 2.2.2 Seja $G(y)=b y^{2 N}+O\left(y^{2 N+1}\right)$ analítica em $\widetilde{\Omega} \operatorname{com} b>0$. Para $y \in\left(\bar{y}_{-}, \bar{y}_{+}\right)$ definamos

$$
\sigma(y)=y-\operatorname{sgn}(y) l_{G}(G(y))
$$

Então $\sigma$ é uma involução estrita analítica tal que $G(y)=G(\sigma(y))$.

Demonstração. Sabemos pelo Lema 2.2.1, que se $\phi(y)=\operatorname{sgn}(y) \sqrt[2 N]{G(y)}$, então

$$
\begin{aligned}
\sigma(y) & =y-\operatorname{sgn}(y) \sqrt[2 N]{G(y)}\left[\mathcal{G}_{1}(G(y))+\sqrt[N]{G(y)} \mathcal{G}_{2}(G(y))+\cdots+\sqrt[N]{(G(y))^{N-1}} \mathcal{G}_{N}(G(y))\right] \\
& =y-\phi(y)\left[\mathcal{G}_{1}(G(y))+\sqrt[N]{G(y)} \mathcal{G}_{2}(G(y))+\cdots+\sqrt[N]{(G(y))^{N-1}} \mathcal{G}_{N}(G(y))\right] \\
& =y-\phi(y) \mathcal{G}_{1}(G(y))-\phi(y) \sqrt[N]{G(y)} \mathcal{G}_{2}(G(y))-\cdots-\phi(y) \sqrt[N]{(G(y))^{N-1}} \mathcal{G}_{N}(G(y))
\end{aligned}
$$

Sejam $\gamma_{k}(y)=\sqrt[N]{G(y)^{k}}, k=0,1, \cdots, N-1$ e $\beta_{i}(y)=\left(\mathcal{G}_{i} \circ G\right)(y), i=1,2, \cdots, N$. $\gamma_{k}$ é analítica em zero, pois 
$\gamma_{k}(y)=\sqrt[N]{G(y)^{k}}=\sqrt[N]{b y^{2 N k}+O\left(y^{2 N k+1}\right)}=\sqrt[N]{y^{2 N k}(b+O(y))}=y^{2 k} \sqrt[N]{b+O(y)}$, e $b>0$. Para $i=1,2, \cdots, N, \beta_{i}(y)=\left(\mathcal{G}_{i} o G\right)(y)$, é composição de funções analíticas, logo é analítica, e também $\phi$ é analítica em zero. Decorre que $\sigma$ é analítica em zero.

Por outro lado,

$$
\sigma(y)= \begin{cases}y-l_{G}(G(y))<0, & \text { se } 0<y<\bar{y}_{+} \\ 0 & \text { se } y=0 \\ y+l_{G}(G(y))>0, & \text { se } \bar{y}_{-}<y<0\end{cases}
$$

Note que $\operatorname{sgn}(\sigma(y))=-\operatorname{sgn}(y)$ e que $\sigma(y)=y_{h-}=G_{-}^{-1}(h)<0$ quando $y=y_{h+}=G_{+}^{-1}(h)>0$ e $\sigma(y)=y_{h_{+}}=G_{+}^{-1}(h)>0$ quando $y=y_{h_{-}}=G_{-}^{-1}(h)<0$, portanto

$$
G(\sigma(y))=G(y), \forall y \in\left(\bar{y}_{-}, \bar{y}_{+}\right) .
$$

De $(2.10)$ vem que $l_{G}(G(y))=l_{G}(G(\sigma(y)))$, e assim

$$
\sigma(\sigma(y))=\sigma(y)-\operatorname{sgn}(\sigma(y)) l_{G}(G(\sigma(y)))=\left(y-\operatorname{sgn}(y) l_{G}(G(y))\right)+\operatorname{sgn}(y) l_{G}(G(y))=y .
$$

Além disso, de (2.8) temos

$$
\begin{aligned}
\sigma^{\prime}(y) & =1-\phi^{\prime}(y)\left[\mathcal{G}_{1}(G(y))+\gamma_{1}(y) \mathcal{G}_{2}(G(y))+\cdots+\gamma_{N-1}(y) \mathcal{G}_{N}(G(y))\right] \\
& +\phi(y)\left[\mathcal{G}_{1}(G(y))+\gamma_{1}(y) \mathcal{G}_{2}(G(y))+\cdots+\gamma_{N-1}(y) \mathcal{G}_{N}(G(y))\right]^{\prime}
\end{aligned}
$$

e portanto, como $\gamma_{k}(0)=0, k=1,2, \cdots, N-1$ e $\phi(0)=0$, obtemos

$$
\sigma^{\prime}(0)=1-\phi^{\prime}(0) \mathcal{G}_{1}(0)=1-\sqrt[2 N]{b} \frac{2}{\sqrt[2 N]{b}}=-1
$$

Portanto $\sigma$ é uma involução estrita.

Observação 2.2.2 Para $l_{G}$ dada no Lema 2.2.1 temos, para $h>0$,

$$
\begin{aligned}
l_{G}^{\prime}(h) & =\frac{1}{2 N \sqrt[2 N]{h^{2 N-1}}}\left[\mathcal{G}_{1}(h)+\sqrt[N]{h} \mathcal{G}_{2}(h)+\cdots+\sqrt[N]{h^{N-1}} \mathcal{G}_{N}(h)\right] \\
& +\sqrt[2 N]{h}\left[\mathcal{G}_{1}^{\prime}(h)+\frac{1}{N \sqrt[N]{h^{N-1}}} \mathcal{G}_{2}(h)+\sqrt[N]{h} \mathcal{G}_{2}^{\prime}(h)+\cdots+\frac{N-1}{N \sqrt[N]{h}} \mathcal{G}_{N}(h)+\sqrt[N]{h^{N-1} h} \mathcal{G}_{N}^{\prime}(h)\right] \\
& =\frac{\mathcal{G}_{1}(h)+2 N h \mathcal{G}_{1}^{\prime}(h)}{2 N \sqrt[2 N]{h^{2 N-1}}}+\frac{3 \mathcal{G}_{2}(h)+2 N h \mathcal{G}_{2}^{\prime}(h)}{2 N \sqrt[2 N]{h^{2 N-3}}}+\cdots+\frac{(2 N-1) \mathcal{G}_{N}(h)+2 N h \mathcal{G}_{N}^{\prime}(h)}{2 N \sqrt[2 N]{h}} \\
& =\frac{B_{1}(h)}{\sqrt[2 N]{h^{2 N-1}}}+\frac{B_{2}(h)}{\sqrt[2 N]{h^{2 N-3}}}+\cdots+\frac{B_{N}(h)}{\sqrt[2 N]{h}}
\end{aligned}
$$


onde $B_{1}, B_{2}, \cdots, B_{N}$ são funções analíticas em zero, com

$$
B_{1}(0)=\frac{\mathcal{G}_{1}(0)}{2 N}=\frac{2}{2 N \sqrt[2 N]{b}}>0 .
$$

Lema 2.2.3 Seja $\Gamma(y)=[\gamma(y)]^{2 N}$, onde $\gamma$ é analitica em zero com $\gamma(0)=0$. Então $\Gamma^{\prime}(0)=\Gamma^{\prime \prime}(0)=\cdots=\Gamma^{(2 N-1)}(0)=0$ e $\Gamma^{(2 N)}(0)=(2 N) !\left[\gamma^{\prime}(0)\right]^{2 N}$.

Em particular, se $L(y)=y \mathcal{G}_{1}\left(y^{2 N}\right)+y^{3} \mathcal{G}_{2}\left(y^{2 N}\right)+\cdots+y^{2 N-1} \mathcal{G}_{N}\left(y^{2 N}\right) \mathrm{com}$ $\mathcal{G}_{1}, \mathcal{G}_{2}, \cdots, \mathcal{G}_{N}$ dadas no Lema 2.2.1 e $G(y)=\left(L^{-1}(y-\sigma(y))\right)^{2 N}$ então $G^{\prime}(0)=G^{\prime \prime}(0)=\cdots=G^{2 N-1}(0)=0$ e $G^{2 N}(0)=2 N !\left[\frac{1-\sigma^{\prime}(0)}{L^{\prime}(0)}\right]^{2 N}$, sendo $\sigma$ involução estrita analítica.

Demonstração. Primeiramente provemos por indução sobre $j$ que para $j=1,2, \cdots, 2 N$,

$$
\Gamma^{(j)}(y)=[\gamma(y)]^{2 N-j}\left\{\frac{(2 N) !}{(2 N-j) !}\left[\gamma^{\prime}(y)\right]^{j}+\gamma(y) \eta_{j}(y)\right\}
$$

para alguma função $\eta_{j}$ analítica em zero.

Para $j=1$ temos $\Gamma^{\prime}(y)=2 N[\gamma(y)]^{2 N-1} \gamma^{\prime}(y)$ e (2.11) vale com $\eta_{1} \equiv 0$.

Suponhamos (2.11) válido para $j, 1 \leq j \leq 2 N-1$. Então

$$
\begin{aligned}
\Gamma^{(j+1)}(y) & =(2 N-j)[\gamma(y)]^{2 N-j-1} \gamma^{\prime}(y)\left\{\frac{(2 N) !}{(2 N-j) !}\left[\gamma^{\prime}(y)\right]^{j}+\gamma(y) \eta_{j}(y)\right\} \\
& +[\gamma(y)]^{2 N-j} \frac{d}{d y}\left\{\frac{(2 N) !}{(2 N-j) !}\left[\gamma^{\prime}(y)\right]^{j}+\gamma(y) \eta_{j}(y)\right\} \\
& =\frac{(2 N) !}{(2 N-(j+1)) !}[\gamma(y)]^{2 N-(j+1)}\left[\gamma^{\prime}(y)\right]^{j+1}+[\gamma(y)]^{2 N-(j+1)}\left\{(2 N-j) \gamma^{\prime}(y) \eta_{j}(y)\right. \\
& \left.+\frac{d}{d y}\left[\frac{(2 N) !}{(2 N-j) !}\left[\gamma^{\prime}(y)\right]^{j}+\gamma(y) \eta_{j}(y)\right]\right\} \gamma(y) \\
& =[\gamma(y)]^{2 N-(j+1)}\left\{\frac{(2 N) !}{(2 N-(j+1)) !}\left[\gamma^{\prime}(y)\right]^{j+1}+\gamma(y) \eta_{j+1}(y)\right\}
\end{aligned}
$$

onde $\eta_{j+1}(y)=(2 N-j) \gamma^{\prime}(y) \eta_{j}(y)+\frac{d}{d y}\left\{\frac{(2 N) !}{(2 N-j) !}\left[\gamma^{\prime}(y)\right]^{j}+\gamma(y) \eta_{j}(y)\right\}$.

Como $\eta_{j+1}$ é analítica em zero segue que (2.11) vale para $j+1$.

Agora que (2.11) está provado é imediato do fato de $\gamma(0)=0$ que

$$
\Gamma^{(j)}(0)=0 \text { para } j=1,2, \cdots, 2 N-1 .
$$

Também, pondo $j=2 N$ em (2.11) e usando novamente que $\gamma(0)=0$ obtemos

$$
\Gamma^{(2 N)}(0)=\frac{(2 N) !}{0 !}\left[\gamma^{\prime}(0)\right]^{2 N}=(2 N) !\left[\gamma^{\prime}(0)\right]^{2 N}
$$


Agora, as afirmações sobre $G$ são imediatas.

Lema 2.2.4 Seja $l(h)=\sqrt[2 N]{h}\left(\mathcal{G}_{1}(h)+\sqrt[N]{h} \mathcal{G}_{2}(h)+\cdots+\sqrt[N]{h^{N-1}} \mathcal{G}_{N}(h)\right)$, com $\mathcal{G}_{i}$ analítica em zero, $i=1,2, \cdots, N$, tal que $\mathcal{G}_{1}(0)>0$ e seja $\sigma$ uma involuçáa estrita analítica. Então existe uma única função analítica $G$ da forma $G(y)=b y^{2 N}+O\left(y^{2 N+1}\right)$, com $b>0$, definida numa vizinhança $W_{0}$ da origem, tal que $l(h)=G_{+}^{-1}(h)-G_{-}^{-1}(h)$ para $h>0$ suficientemente pequeno e $\sigma(y)=y-\operatorname{sgn}(y) l(G(y))$ em $W_{0}$.

Demonstração. A função $L(s)=s \mathcal{G}_{1}\left(s^{2 N}\right)+s^{3} \mathcal{G}_{2}\left(s^{2 N}\right)+\cdots+s^{2 N-1} \mathcal{G}_{N}\left(s^{2 N}\right)$, é analítica, pois é composição de funções analíticas.

Seja $\mathcal{L}\left(s^{2 N}\right)=\mathcal{G}_{1}\left(s^{2 N}\right)+s^{2} \mathcal{G}_{2}\left(s^{2 N}\right)+\cdots+s^{2 N-2} \mathcal{G}_{N}\left(s^{2 N}\right)$. Assim $L(s)=s \mathcal{L}\left(s^{2 N}\right)$, com $\mathcal{L}(0)>0$; além disso $L^{\prime}(s)=\mathcal{L}\left(s^{2 N}\right)+2 N \mathcal{L}^{\prime}\left(s^{2 N}\right) s^{2 N}$, donde $L^{\prime}(0)=\mathcal{L}(0)>0$. Assim, existe uma vizinhança $V=(-\sqrt[2 N]{\bar{h}}, \sqrt[2 N]{\bar{h}})$ de zero onde $L$ é estritamente crescente e $\left.L\right|_{V}$ possui uma inversa $L^{-1}$ analítica definida em $W=L(V)$ [Rud99], [Rud76]. Como $L^{-1}(0)=L(0)=0$ e ambas são crescentes temos $\operatorname{sgn}(L(s))=\operatorname{sgn}(s)$ e $\operatorname{sgn}\left(L^{-1}(s)\right)=\operatorname{sgn}(s)$.

Seja $W_{0}$ vizinhança aberta de zero tal que $y \in W_{0}$ se, e somente se, $y-\sigma(y) \in W$. Para $y \in W_{0}$ definimos $G(y)=\left(L^{-1}(y-\sigma(y))\right)^{2 N}$. Claramente $G$ é analítica e $G(y) \in$ $[0, \bar{h}), \forall y \in W_{0}$.

Pelo Lema, 2.2.3, $G^{\prime}(0)=G^{\prime \prime}(0)=G^{\prime \prime \prime}(0)=\cdots=G^{2 N-1}(0)=0 \mathrm{e}$ $G^{(2 N)}(0)=(2 N) !\left[\frac{1-\sigma^{\prime}(0)}{L^{\prime}(0)}\right]^{2 N}>0$. Então $G$ tem a forma pretendida. Portanto, como

$$
\begin{aligned}
l\left(k^{2 N}\right) & =\sqrt[2 N]{k^{2 N}}\left[\mathcal{G}_{1}\left(k^{2 N}\right)+\sqrt[N]{k^{2 N}} \mathcal{G}_{2}\left(k^{2 N}\right)+\cdots+\sqrt[N]{\left(k^{2 N}\right)^{N-1}} \mathcal{G}_{N}\left(k^{2 N}\right)\right] \\
& =\operatorname{sgn}(k) k\left[\mathcal{G}_{1}\left(k^{2 N}\right)+k^{2} \mathcal{G}_{2}\left(k^{2 N}\right)+\cdots+k^{2 N-2} \mathcal{G}_{N}\left(k^{2 N}\right)\right]=\operatorname{sgn}(k) L(k)
\end{aligned}
$$

temos que

$$
\begin{aligned}
l(G(y)) & =l\left(\left(L^{-1}(y-\sigma(y))\right)^{2 N}\right) \\
& =\operatorname{sgn}\left(L^{-1}(y-\sigma(y))\right) L\left(L^{-1}(y-\sigma(y))\right) \\
& =\operatorname{sgn}(y-\sigma(y))(y-\sigma(y))=\operatorname{sgn}(y)(y-\sigma(y)) .
\end{aligned}
$$

Logo,

$$
\sigma(y)=y-\operatorname{sgn}(y) l(G(y)) \text { em } W_{0}
$$


Por outro lado, da definição de $G$ temos

$$
\begin{aligned}
G(\sigma(y)) & =\left(L^{-1}(\sigma(y)-\sigma(\sigma(y)))\right)^{2 N}=\left(L^{-1}(\sigma(y)-y)\right)^{2 N} \\
& =\left(L^{-1}(-(y-\sigma(y)))\right)^{2 N}=\left(-L^{-1}(y-\sigma(y))\right)^{2 N}=\left(L^{-1}(y-\sigma(y))\right)^{2 N}=G(y)
\end{aligned}
$$

Portanto, denotando por $y_{h+}=G_{+}^{-1}(h)$ e $y_{h-}=G_{-}^{-1}(h)$ temos $\sigma\left(y_{h+}\right)=y_{h_{-}}$, e observando que $l(G(y))=\operatorname{sgn}(y-\sigma(y))(y-\sigma(y))=(y-\sigma(y))$ se $y \geq 0$ vem que

$$
l(h)=l\left(G\left(y_{h+}\right)\right)=y_{h_{+}}-\sigma\left(y_{h+}\right)=G_{+}^{-1}(h)-G_{-}^{-1}(h) .
$$

Note que se $l(G(x))=l(\widetilde{G}(x))$, então $L(\sqrt[2 N]{G(x)})=L(\sqrt[2 N]{\widetilde{G}(x)})$ logo a unicidade da $G$ decorre do fato de $L$ ser estritamente crescente em $V$. 
Lema 2.2.5 Sejam $\rho_{0}, \rho_{1}, \cdots, \rho_{k-1}$ e $\bar{\rho}_{0}, \overline{\rho_{1}}, \cdots, \bar{\rho}_{k-1}$ analíticas em $[0, \epsilon)$ tais que

$$
\begin{gathered}
\phi(s)=\rho_{0}(s)+\sqrt[k]{s} \rho_{1}(s)+\sqrt[k]{s^{2}} \rho_{2}(s)+\cdots+\sqrt[k]{s^{k-1}} \rho_{k-1}(s) e \\
\bar{\phi}(s)=\bar{\rho}_{0}(s)+\sqrt[k]{s} \bar{\rho}_{1}(s)+\sqrt[k]{s^{2}} \bar{\rho}_{2}(s)+\cdots+\sqrt[k]{s^{k-1}} \bar{\rho}_{k-1}(s)
\end{gathered}
$$

coincidam em $[0, \epsilon)$. Então $\rho_{j} \equiv \bar{\rho}_{j}$, em $[0, \epsilon), j=0,1,2, \cdots, k-1$.

Demonstração. Seja $0<\delta<\epsilon$ tal que

$$
\rho_{j}(s)=\sum_{i=0}^{\infty} a_{i}^{j} s^{i} \text { e } \bar{\rho}_{j}(s)=\sum_{i=0}^{\infty} \bar{a}_{i}^{j} s^{i}, \quad j=0,1, \cdots, k-1 .
$$

Então $\Phi$ definida em $[0, \delta)$ por

$$
\begin{aligned}
\Phi(s) & =\phi\left(s^{k}\right)-\bar{\phi}\left(s^{k}\right) \\
& =\left[\rho_{0}\left(s^{k}\right)-\bar{\rho}_{0}\left(s^{k}\right)\right]+s\left[\rho_{1}\left(s^{k}\right)-\overline{\rho_{1}}\left(s^{k}\right)\right]+\cdots+s^{k-1}\left[\rho_{k-1}\left(s^{k}\right)-\bar{\rho}_{k-1}\left(s^{k}\right)\right]
\end{aligned}
$$

é analítica, $\Phi(s)=\sum_{n=0}^{\infty} C_{n} s^{n} \operatorname{com} C_{k i+j}=a_{i}^{j}-\bar{a}_{i}^{j}, 0 \leq j \leq k-1, i \in \mathbb{N}$, e $\Phi$ é identicamente nula. Logo, $\rho_{j} \equiv \bar{\rho}_{j}$ em $[0, \delta)$ e pelo princípio do prolongamento analítico, [Lim76], [Rud76] $\rho_{j} \equiv \bar{\rho}_{j}$ onde ambas estiverem definidas.

Lema 2.2.6 Sejam $F(x)=a x^{2}+O\left(x^{2}\right) \operatorname{com} a>0$ e $G(y)=b y^{2 N}+O\left(y^{2 N+1}\right) \operatorname{com} b>0$, ambas analíticas em zero. Então existem $T_{1}, T_{2}, T_{3}, \cdots, T_{N}$ funções analíticas em zero com $T_{1}(0)>0$ tal que a função período para o sistema (1.2) é dada por

$$
T(h)=\frac{1}{\sqrt[2 N]{h^{N-1}}}\left[T_{1}(h)+\sqrt[2 N]{h^{2}} T_{2}(h)+\sqrt[2 N]{h^{4}} T_{3}(h)+\cdots+\sqrt[2 N]{h^{2 N-2}} T_{N}(h)\right] .
$$

Demonstração. Usando o Lema 2.2.1 e a Observação 2.2.2, temos:

$$
l_{G}^{\prime}(h)=\frac{B_{1}(h)}{\sqrt[2 N]{h^{2 N-1}}}+\frac{B_{2}(h)}{\sqrt[2 N]{h^{2 N-3}}}+\cdots+\frac{B_{N}(h)}{\sqrt[2 N]{h}}
$$

onde $B_{1}, B_{2}, \cdots, B_{N}$ são funções analíticas em zero com $B_{1}(0)>0$, e do Lema 1.2.1 tem-se $l_{F}^{\prime}(h)=\frac{A(h)}{\sqrt{h}} \operatorname{com} A(0)>0$, logo, pela Proposição 1.2.2, a função período é dada por:

$$
\begin{aligned}
T(h) & =\left(l_{F}^{\prime} * l_{G}^{\prime}\right)=\int_{0}^{h} l_{F}^{\prime}(h-z) l_{G}^{\prime}(z) d z \\
& =\int_{0}^{h} \frac{A(h-z)}{\sqrt{h-z}}\left(\frac{B_{1}(z)}{\sqrt[2 N]{z^{2 N-1}}}+\frac{B_{2}(z)}{\sqrt[2 N]{z^{2 N-3}}}+\cdots+\frac{B_{N}(z)}{\sqrt[2 N]{z}}\right) d z
\end{aligned}
$$


Façamos a mudança de variável $z=h \cos ^{2} \theta$. A expressão anterior fica:

$$
\begin{aligned}
T(h)=2 \int_{0}^{\frac{\pi}{2}} & \frac{A\left(h \operatorname{sen}^{2} \theta\right)}{\sqrt{h \operatorname{sen}^{2} \theta}}\left(\frac{B_{1}\left(h \cos ^{2} \theta\right)}{\sqrt[2 N]{\left(h \cos ^{2} \theta\right)^{2 N-1}}}+\frac{B_{2}\left(h \cos ^{2} \theta\right)}{\sqrt[2 N]{\left(h \cos ^{2} \theta\right)^{2 N-3}}}+\cdots+\frac{B_{N}\left(h \cos ^{2} \theta\right)}{\sqrt[2 N]{h \cos ^{2} \theta}}\right) \\
& h \operatorname{sen}^{2} \theta \cos ^{2} \theta d \theta
\end{aligned}
$$

Sejam $A(h)=\sum_{n=0}^{\infty} a_{n} h^{n}$ e $B_{q}(h)=\sum_{n=0}^{\infty} b_{n}^{q} h^{n}$ em $[0, \epsilon), \quad q=1,2, \cdots, N . \quad$ Deste modo,

$$
\begin{aligned}
& T(h)= \\
& 2 \int_{0}^{\frac{\pi}{2}} \sum_{n=0}^{\infty} a_{n}\left(h \operatorname{sen}^{2} \theta\right)^{n}\left[\frac{\sum_{n=0}^{\infty} b_{n}^{1}\left(h \cos ^{2} \theta\right)^{n}}{\sqrt[2 N]{\left(h \cos ^{2} \theta\right)^{N-1}}}+\frac{\sum_{n=0}^{\infty} b_{n}^{2}\left(h \cos ^{2} \theta\right)^{n}}{\sqrt[2 N]{\left(h \cos ^{2} \theta\right)^{N-3}}}+\cdots+\frac{\sum_{n=0}^{\infty} b_{n}^{N}\left(h \cos ^{2} \theta\right)^{n}}{\sqrt[2 N]{\left(h \cos ^{2} \theta\right)^{1-N}}}\right] d \theta
\end{aligned}
$$

Pelo Lema 1.2.5 segue que

$$
\begin{aligned}
& T(h)=2 \int_{0}^{\frac{\pi}{2}} \sum_{k=0}^{\infty}\left(\sum_{i+j=k} a_{i} b_{j}^{1} \frac{\left(\operatorname{sen}^{2 i} \theta \cos ^{2 j} \theta\right)}{\sqrt[2 N]{\left(h \cos ^{2} \theta\right)^{N-1}}}\right) h^{k} \\
& +\sum_{k=0}^{\infty}\left(\sum_{i+j=k} a_{i} b_{j}^{2} \frac{\left(\operatorname{sen}^{2 i} \theta \cos ^{2 j} \theta\right)}{\sqrt[2 N]{\left(h \cos ^{2} \theta\right)^{N-3}}}\right) h^{k}+\cdots \\
& +\sum_{k=0}^{\infty}\left(\sum_{i+j=k} a_{i} b_{j}^{N} \frac{\left(\operatorname{sen}^{2 i} \theta \cos ^{2 j} \theta\right)}{\sqrt[2 N]{\left(h \cos ^{2} \theta\right)^{1-N}}}\right) h^{k} d \theta \\
& =\frac{1}{\sqrt[2 N]{h^{N-1}}}\left[\sum_{k=0}^{\infty}\left(\sum_{i+j=k} a_{i} b_{j}^{1}\left(2 \int_{0}^{\frac{\pi}{2}} \operatorname{sen}^{2 i} \theta \cos ^{2 j-\frac{N-1}{N}} \theta d \theta\right)\right) h^{k}\right. \\
& +\sqrt[2 N]{h^{2}} \sum_{k=0}^{\infty}\left(\sum_{i+j=k} a_{i} b_{j}^{2}\left(2 \int_{0}^{\frac{\pi}{2}} \operatorname{sen}^{2 i} \theta \cos ^{2 j-\frac{N-3}{N}} \theta d \theta\right)\right) h^{k}+\cdots \\
& \left.+\sqrt[2 N]{h^{2 N-2}} \sum_{k=0}^{\infty}\left(\sum_{i+j=k} a_{i} b_{j}^{N}\left(2 \int_{0}^{\frac{\pi}{2}} \operatorname{sen}^{2 i} \theta \cos ^{2 j-\frac{1-N}{N}} \theta d \theta\right)\right) h^{k}\right] \\
& =\frac{1}{\sqrt[2 N]{h^{N-1}}}\left[\sum_{k=0}^{\infty}\left(\sum_{i+j=k} a_{i} b_{j}^{1} I_{i, j}^{1}\right) h^{k}+\sqrt[2 N]{h^{2}} \sum_{k=0}^{\infty}\left(\sum_{i+j=k} a_{i} b_{j}^{2} I_{i, j}^{2}\right) h^{k}+\cdots\right. \\
& \left.+\sqrt[2 N]{h^{2 N-2}} \sum_{k=0}^{\infty}\left(\sum_{i+j=k} a_{i} b_{j}^{N} I_{i, j}^{N}\right) h^{k}\right] \\
& =\frac{1}{\sqrt[2 N]{h^{N-1}}}\left[\sum_{n=0}^{\infty} t_{n}^{1} h^{n}+\sqrt[2 N]{h^{2}} \sum_{n=0}^{\infty} t_{n}^{2} h^{n}+\cdots+\sqrt[2 N]{h^{2 N-2}} \sum_{n=0}^{\infty} t_{n}^{N} h^{n}\right]
\end{aligned}
$$


onde

$$
I_{i, j}^{q}=2 \int_{0}^{\frac{\pi}{2}} \operatorname{sen}^{2 i} \theta \cos ^{2 j-\frac{N-2 q+1}{N}} \theta d \theta \text { e } t_{n}^{q}=\sum_{i+j=n} a_{i} b_{j}^{q} I_{i, j}^{q}, \quad q=1,2, \cdots, N .
$$

Logo, as funções analíticas em zero

$$
T_{q}(h)=\sum_{n=0}^{\infty} t_{n}^{q} h^{n}, \quad q=1,2, \cdots, N
$$

são tais que

$$
T(h)=\frac{1}{\sqrt[2 N]{h^{N-1}}}\left[T_{1}(h)+\sqrt[2 N]{h^{2}} T_{2}(h)+\sqrt[2 N]{h^{4}} T_{3}(h)+\cdots+\sqrt[2 N]{h^{2 N-2}} T_{N}(h)\right] \text { em }(0, \epsilon) .
$$

Também, $T_{1}(0)=t_{0}^{1}=a_{0} b_{0}^{1} I_{0,0}^{1}=A(0) B_{1}(0) I_{0,0}^{1}>0$.

\section{Proposição 2.2.1 Seja}

$T(h)=\frac{1}{\sqrt[2 N]{h^{N-1}}}\left[T_{1}(h)+\sqrt[2 N]{h^{2}} T_{2}(h)+\sqrt[2 N]{h^{4}} T_{3}(h)+\cdots+\sqrt[2 N]{h^{2 N-2}} T_{N}(h)\right]$, onde $T_{1}, T_{2}$, $T_{3}, \cdots, T_{N}$ são funções analíticas em zero e $T_{1}(0)>0$. Seja A analítica em zero tal que $A(0)>0$. Então a equação integral

$$
T(h)=\int_{0}^{h} \frac{A(h-z)}{\sqrt{h-z}}\left(\frac{B_{1}(h)}{\sqrt[2 N]{h^{2 N-1}}}+\frac{B_{2}(h)}{\sqrt[2 N]{h^{2 N-3}}}+\cdots+\frac{B_{N}(h)}{\sqrt[2 N]{h}}\right) d z
$$

tem uma única solução dada por $N$ funções analíticas $B_{1}, B_{2}, B_{3}, \cdots, B_{N}$ com $B_{1}(0)>0$.

Observação: A unicidade no enunciado anterior significa que se $B_{1}, B_{2}, \cdots, B_{N}$ são analíticas definidas em $[0, r)$ satisfazendo $(2.15)$ e $\widetilde{B}_{1}, \widetilde{B}_{2}, \cdots, \widetilde{B}_{N}$ são analíticas em $[0, \tilde{r})$ satisfazendo (2.15) então $B_{k} \equiv \widetilde{B}_{k}$ em $[0, r) \cap[0, \tilde{r}), k=1,2, \cdots, N$.

Demonstração. Seja $R>0$ tal que $A(h)=\sum_{k=0}^{\infty} a_{k} h^{k}, T_{1}(h)=\sum_{n=0}^{\infty} t_{n}^{1} h^{n}, T_{2}(h)=\sum_{n=0}^{\infty} t_{n}^{2} h^{n}, \cdots, T_{N}(h)=\sum_{n=0}^{\infty} t_{n}^{N} h^{n}$, em $[0, R]$.

Comecemos provando a unicidade de solução. Sejam $B_{1}, B_{2}, \cdots, B_{N}$ soluções analíticas em $[0, r)$ e suponhamos que $B_{1}(h)=\sum_{n=0}^{\infty} b_{n}^{1} h^{n}, B_{2}(h)=\sum_{n=0}^{\infty} b_{n}^{2} h^{n}, \cdots, B_{N}(h)=\sum_{n=0}^{\infty} b_{n}^{N} h^{n}$ em $[0, \epsilon)$ para algum $\epsilon>0$. Podemos supor sem perda de generalidade $\epsilon \leq R$.

Como na prova do Lema 2.2.6, fazendo a mudança de variáveis $z=h \cos ^{2} \theta$ em (2.15) e usando o Lema 1.2.5 podemos repetir as passagens feitas em (2.12), (2.13) e (2.14), e 
assim,

$$
\begin{aligned}
T(h) & =\int_{0}^{h} \frac{A(h-z)}{\sqrt{h-z}}\left(\frac{B_{1}(h)}{\sqrt[2 N]{h^{2 N-1}}}+\frac{B_{2}(h)}{\sqrt[2 N]{h^{2 N-3}}}+\cdots+\frac{B_{N}(h)}{\sqrt[2 N]{h}}\right) d z \\
& =\frac{1}{\sqrt[2 N]{h^{N-1}}}\left[\sum_{n=0}^{\infty}\left(\sum_{i+j=n} a_{i} b_{j}^{1} I_{i, j}^{1}\right) h^{n}+\sqrt[2 N]{h^{2}} \sum_{n=0}^{\infty}\left(\sum_{i+j=n} a_{i} b_{j}^{2} I_{i, j}^{2}\right) h^{n}+\cdots\right. \\
& \left.+\sqrt[2 N]{h^{2 N-2}} \sum_{n=0}^{\infty}\left(\sum_{i+j=n} a_{i} b_{j}^{N} I_{i, j}^{N}\right) h^{n}\right]
\end{aligned}
$$

onde

$$
I_{i, j}^{q}=2 \int_{0}^{\frac{\pi}{2}} \operatorname{sen}^{2 i} \theta \cos ^{2 j-\frac{N-2 q+1}{N}} \theta d \theta .
$$

Agora usando o Lema 2.2.5, teremos:

$$
\left\{\begin{array}{l}
T_{1}(h)=\sum_{n=0}^{\infty}\left(\sum_{i+j=n} a_{i} b_{j}^{1} I_{i, j}^{1}\right) h^{n}, \text { ou seja, } t_{n}^{1}=\sum_{i+j=n} a_{i} b_{j}^{1} I_{i, j}^{1} \\
T_{2}(h)=\sum_{n=0}^{\infty}\left(\sum_{i+j=n} a_{i} b_{j}^{2} I_{i, j}^{2}\right) h^{n}, \text { ou seja, } t_{n}^{2}=\sum_{i+j=n} a_{i} b_{j}^{2} I_{i, j}^{2} \\
\vdots \\
T_{N}(h)=\sum_{n=0}^{\infty}\left(\sum_{i+j=n} a_{i} b_{j}^{N} I_{i, j}^{N}\right) h^{n}, \text { ou seja, } t_{n}^{N}=\sum_{i+j=n} a_{i} b_{j}^{N} I_{i, j}^{N}
\end{array}\right.
$$

Observando que $a_{0}=A(0) \neq 0$, a unicidade de $b_{j}^{N}$ segue pelo mesmo argumento aplicado a (1.17) na demonstração da Proposição 1.2.3, pois o sistema linear

$$
t_{n}^{q}=\sum_{i+j=n} a_{i} b_{j}^{q} I_{i, j}^{q}
$$

onde $I_{i, j}^{q}=2 \int_{0}^{\frac{\pi}{2}} \operatorname{sen}^{2 i} \theta \cos ^{2 j-\frac{N-2 q+1}{N}} \theta d \theta$, é possível e determinado em $b_{j}^{q}, j \in \mathbb{N}$. Disto segue a unicidade local de $B_{1}, B_{2}, \cdots, B_{N}$.

A unicidade global segue então do princípio do prolongamento analítico, [Lim76].

Passemos agora a provar a existência de solução.

Sejam $\left(b_{j}^{q}\right)_{j \in \mathbb{N}}, q=1,2, \cdots, N$, definidos por (2.17).

Para mostrarmos a existência de solução $B_{1}, B_{2}, \cdots, B_{q}$ numa vizinhança de zero, basta mostrarmos que as séries $\sum_{n=0}^{\infty} b_{n}^{q} z^{n}$ convergem em alguma vizinhança de zero, pois 
então as passagens em (2.16) serão satisfeitas, já que, segundo o Lema 1.2.5, as passagens em (2.12), (2.13) e (2.14) ficam satisfeitas.

Suponhamos sem perda de generalidade $a_{0}=1$.

Já que as séries de potências $\sum a_{n} h^{n}$ e $\sum(n+1) t_{n}^{q} h^{n}$ convergem numa vizinhança de zero podemos tomar $C>0$, como na Proposição 1.2.3, de modo que

$$
\left|a_{n}\right| \leq C^{n} \text { e }\left|t_{n}^{q}\right| \leq \frac{C^{n+1}}{4 N} \frac{I_{0,0}^{q}}{2 N(n+1)} .
$$

Então pelo Fato A.0.20 teremos $\left|t_{n}^{q}\right| \leq \frac{C^{n+1}}{4 N} I_{0, n}^{q}$.

$$
\text { Afirmamos que }\left|b_{k}^{q}\right| \leq\left\{\begin{array}{cl}
(k+1) C^{k+1} & \text { se } k \neq 0 \\
\frac{C}{N} & \text { se } k=0 .
\end{array}\right.
$$

Faremos a demonstração por indução sobre $k$.

Para $k=0$ temos, por (2.18), $b_{0}^{q}=\frac{t_{0}^{q}}{I_{0,0}^{q}}$, portanto, $\left|b_{0}^{q}\right|=\frac{\left|t_{0}^{q}\right|}{I_{0,0}^{q}}=\frac{C I_{0,0}^{q}}{4 N I_{0,0}^{q}}<\frac{C}{N}$.

Para $k=1$ temos, por $(2.18), b_{1}^{q}=\frac{t_{1}^{q}-a_{1} b_{0}^{q} I_{1,0}^{q}}{I_{0,1}^{q}}$, e portanto

$$
\left|b_{1}^{q}\right| \leq \frac{\left|t_{1}^{q}\right|+\left|a_{1}\right|\left|b_{0}^{q}\right| I_{1,0}^{q}}{I_{0,1}^{q}} \leq \frac{C^{2} I_{0,1}^{q}}{4 N I_{0,1}^{q}}+\frac{C^{2} I_{1,0}^{q}}{N I_{0,1}^{q}}
$$

Pelo Fato A.0.11, $\frac{I_{1,0}^{q}}{I_{0,1}^{q}}=\frac{N}{2 q-1}$, assim $\left|b_{1}^{q}\right| \leq \frac{C^{2}}{4 N}+\frac{C^{2}}{2 q-1}<2 C^{2}$.

Para $k=2$ temos, por $(2.18), b_{2}^{q}=\frac{t_{2}^{q}-a_{1} b_{1}^{q} I_{1,1}^{q}-a_{2} b_{0}^{q} I_{2,0}^{q}}{I_{0,2}^{q}}$, e portanto usando Fato A.0.12 que garante que $\frac{I_{1,1}^{q}}{I_{0,2}^{q}} \leq \frac{1}{2}$ e $\frac{I_{2,0}^{q}}{I_{0,2}^{q}} \leq \frac{3 N}{2(2 q-1)}$, temos

$$
\begin{aligned}
\left|b_{2}^{q}\right| & \leq \frac{\left|t_{2}^{q}\right|+\left|a_{1}\right|\left|b_{1}^{q}\right| I_{1,1}^{q}+\left|a_{2}\right|\left|b_{0}^{q}\right| I_{2,0}^{q}}{I_{0,2}^{q}} \\
& \leq \frac{C^{3} I_{0,2}^{q}}{4 N I_{0,2}^{q}}+\frac{C 2 C^{2} I_{1,1}^{q}}{I_{0,2}^{q}}+\frac{C^{2} C I_{2,0}^{q}}{N I_{0,2}^{q}} \\
& \leq C^{3}\left[\frac{1}{4 N}+1+\frac{3}{2(2 q-1)}\right]<3 C^{3} .
\end{aligned}
$$

Para $k=3$ temos, por $(2.18), b_{2}^{q}=\frac{t_{3}^{q}-a_{1} b_{2}^{q} I_{1,2}^{q}-a_{2} b_{1}^{q} I_{2,1}^{q}-a_{3} b_{0}^{q} I_{3,0}^{q}}{I_{0,3}^{q}}$, e portanto usando $\frac{I_{1,2}^{q}}{I_{0,3}^{q}} \leq \frac{1}{4}$ e $\frac{I_{2,1}^{q}}{I_{0,3}^{q}} \leq \frac{3}{8}$, (Fato A.0.13) e também o Fato A.0.15, que garante que 
$\frac{I_{3,0}^{q}}{I_{0,3}^{q}} \leq \frac{15 N}{8(2 q-1)}<\frac{15 N}{8}$, temos

$$
\begin{aligned}
\left|b_{3}^{q}\right| & \leq \frac{\left|t_{3}^{q}\right|+\left|a_{1}\right|\left|b_{2}^{q}\right| I_{1,2}^{q}+\left|a_{2}\right|\left|b_{1}^{q}\right| I_{2,1}^{q}+\left|a_{3}\right|\left|b_{0}^{q}\right| I_{3,0}^{q}}{I_{0,3}^{q}} \\
& \leq \frac{C^{4} I_{0,3}^{q}}{4 N I_{0,3}^{q}}+\frac{C 3 C^{3} I_{1,2}^{q}}{I_{0,3}^{q}}+\frac{C^{2} 2 C^{2} I_{2,1}^{q}}{I_{0,3}^{q}}+\frac{C^{3} C I_{3,0}^{q}}{N I_{0,3}^{q}} \\
& \leq C^{4}\left[\frac{1}{4}+\frac{3}{4}+\frac{6}{8}+\frac{15}{8}\right]=C^{4} \frac{29}{8}<4 C^{4}
\end{aligned}
$$

Suponhamos a desigualdade válida para $k=0,1,2, \cdots, n-1$ onde $n \geq 4$.

Então por (2.18) temos

$$
\begin{gathered}
t_{n}^{q}=a_{0} b_{n}^{q} I_{0, n}^{q}+a_{1} b_{n-1}^{q} I_{1, n-1}^{q}+\cdots+a_{n-1} b_{1}^{q} I_{n-1,1}^{q}+a_{n} b_{0}^{q} I_{n, 0}^{q} \text { donde } \\
b_{n}^{q}=\frac{t_{n}^{q}-a_{1} b_{n-1}^{q} I_{1, n-1}^{q}-\cdots-a_{n-1} b_{1}^{q} I_{n-1,1}^{q}-a_{n} b_{0}^{q} I_{n, 0}^{q}}{I_{0, n}^{q}} .
\end{gathered}
$$

Logo

$$
\left|b_{n}^{q}\right| \leq \frac{\left|t_{n}^{q}\right|+\left|a_{1}\right|\left|b_{n-1}^{q}\right| I_{1, n-1}^{q}+\cdots+\left|a_{n-1}\right|\left|b_{1}^{q}\right| I_{n-1,1}^{q}+\left|a_{n}\right|\left|b_{0}^{q}\right| I_{n, 0}^{q}}{I_{0, n}^{q}} .
$$

Pelo Fato A.0.14, se $2 \leq j \leq n-2$ temos $I_{j, n-j}^{q} \leq \frac{3}{4} I_{1, n-1}^{q}$. Sabemos ainda pelo Fato A.0.15 que $\frac{I_{n, 0}^{q}}{I_{0, n}^{q}} \leq \frac{15}{48} \frac{N}{2 q-1}(2 n-1)$ se $n \geq 4$, pelo Fato A.0.17 que $I_{n-1,1}^{q}=\frac{2 q-1}{N(2 n-1)} I_{n, 0}^{q}$ se $n \geq 1$, e pelo Fato A.0.18 que $\frac{I_{1, n-1}^{q}}{I_{0, n}^{q}} \leq \frac{1}{2 n-2}$ se $n \geq 2$. Agora substituindo esses 
resultados e as hipóteses de indução em (2.19) teremos:

$$
\begin{aligned}
\left|b_{n}^{q}\right| & \leq \frac{\left|t_{n}^{q}\right|}{I_{0, n}^{q}}+\left|a_{1}\right|\left|b_{n-1}^{q}\right| \frac{I_{1, n-1}^{q}}{I_{0, n}^{q}}+\left(\left|a_{2}\right|\left|b_{n-2}^{q}\right|+\cdots+\left|a_{n-3}\right|\left|b_{3}\right|+\left|a_{n-2}\right|\left|b_{2}^{q}\right|\right) \frac{\frac{3}{4} I_{1, n-1}^{q}}{I_{0, n}^{q}} \\
& +\left(\left|a_{n-1}\right|\left|b_{1}^{q}\right| \frac{2 q-1}{N(2 n-1)}+\left|a_{N}\right|\left|b_{0}^{q}\right|\right) \frac{I_{n, 0}^{q}}{I_{0, n}^{q}} \\
& \leq \frac{I_{0, n}^{q} C^{n+1}}{4 N I_{0, n}^{q}}+\left(C^{2}(n-1) C^{n-1}+\cdots+C^{n-3} 4 C^{4}+C^{n-2} 3 C^{3}\right) \frac{3}{4} \frac{1}{2 n-2} \\
& +\left(C^{n-1} 2 C^{2} \frac{2 q-1}{N(2 n-1)}+C^{n} \frac{C}{N}\right) \frac{15}{40} \frac{N}{2 q-1}(2 n-1)+C n C^{n} \frac{1}{2 n-2} \\
& =C^{n+1}\left[\frac{1}{4 N}+\frac{n}{2(n-1)}+(n+2) \frac{(n-3)}{2} \frac{3}{8(n-1)}+\frac{30}{48}+\frac{15}{48(2 q-1)}(2 n-1)\right] \\
& \stackrel{(*)}{\leq} C^{n+1}\left[\frac{1}{4}+\frac{1}{2} \frac{4}{3}+\frac{3}{16}(n+2)+\frac{30}{48}+\frac{15}{48}(2 n-1)\right] \\
& \leq C^{n+1}\left[\frac{3 n}{16}+\frac{30 n}{48}+\frac{11}{12}+\frac{6}{16}+\frac{30}{48}-\frac{15}{48}\right]=C^{n+1}\left[\frac{39 n+29}{48}+1\right] \\
& \stackrel{(*)}{\leq} C^{n+1}\left[\frac{39 n+8 n}{48}+1\right] \leq C^{n+1}[n+1],
\end{aligned}
$$

note que em $\left(^{*}\right)$ usamos que $n \geq 4$.

Assim a afirmação se verifica. Por fim, como $\left|b_{n}^{q}\right| \leq(n+1) C^{n+1}$, a série $\sum_{n=0}^{\infty} b_{n}^{q} h^{n}$ converge no intervalo $\left(-\frac{1}{C}, \frac{1}{C}\right)$. Claramente, $B(z)=\sum_{n=0}^{\infty} b_{n}^{q} z^{n}$ satisfaz (2.15).

Lema 2.2.7 Seja $l(h)=\sqrt[2 N]{h}\left(\mathcal{G}_{1}(h)+\sqrt[N]{h} \mathcal{G}_{2}(h)+\cdots+\sqrt[N]{h^{N-1}} \mathcal{G}_{N}(h)\right)$ com $\mathcal{G}_{i}$ analíticas em zero, $i=1,2, \cdots, N$, tal que $\mathcal{G}_{1}(0)>0$, e seja $L(y)=y \mathcal{G}_{1}\left(y^{2 N}\right)+y^{3} \mathcal{G}_{2}\left(y^{2 N}\right)+\cdots+y^{2 N-1} \mathcal{G}_{N}\left(y^{2 N}\right)$. Dadas $G$ analítica em zero, da forma $G(y)=b y^{2 N}+O\left(y^{2 N+1}\right), c o m b>0$ tal que $l_{G}=l$, e $\sigma(y)=y-\operatorname{sgn}(y) l(G(y))$, então $G(y)=\left[L^{-1}(y-\sigma(y))\right]^{2 N}$ numa vizinhança de zero.

Demonstração. Pelo Lema 2.2.2, $\sigma$ é uma involução estrita analítica. Então pelo Lema 2.2.4 decorre que existe uma única função analítica $\widetilde{G}$ definida numa vizinhança $W_{0}$ da origem, da forma $\widetilde{G}(y)=\tilde{b} y^{2 N}+O\left(y^{2 N+1}\right)$ tal que $l(h)=\widetilde{G}_{+}^{-1}(h)-\widetilde{G}_{-}^{-1}(h)=l_{\widetilde{G}}(h)$ para $h>0$ suficientemente pequeno, e $\sigma(y)=y-\operatorname{sgn}(y) l(\widetilde{G}(y))$ em $W_{0}$.

Da unicidade de $\widetilde{G}$ e de nossas hipóteses segue que $G=\widetilde{G}$ onde ambas estiverem definidas. 
Da construção de $\widetilde{G}$ na prova da Lema 2.2 .4 decorre que $G(y)=\widetilde{G}(y)=\left[L^{-1}(y-\sigma(y))\right]^{2 N}$ numa vizinhança da origem.

\subsubsection{Resultado principal}

Nesta subseção apresentaremos a generalização de um dos principais resultados de [CGM00], que é o Teorema 1.3.1. O outro resultado importante de [CGM00], o Teorema 1.3.2, não faz sentido neste contexto com $N>1$. Voltaremos a tratar disso no capítulo 3.

Teorema 2.2.1 Seja $T(h)=\frac{1}{\sqrt[2 N]{h^{N-1}}}\left[T_{1}(h)+\sqrt[2 N]{h^{2}} T_{2}(h)+\cdots+\sqrt[2 N]{h^{2 N-2}} T_{N}(h)\right]$, onde $T_{1}, T_{2}, T_{3}, \cdots, T_{N}$ são funções analíticas em zero e $T_{1}(0)>0$ e seja $F(x)=a x^{2}+O\left(x^{3}\right)$ função analítica em zero com $a>0$. Seja $l_{F}(h)=F_{+}^{-1}(h)-F_{-}^{-1}(h), h \in[0, \bar{h}[$. Então as afirmações abaixo são verdadeiras:

(i) Existe uma única $l(h)=\sqrt[2 N]{h}\left[\mathcal{G}_{1}(h)+\sqrt[N]{h} \mathcal{G}_{2}(h)+\cdots+\sqrt[N]{h^{N-1}} \mathcal{G}_{N}(h)\right]$, com $\mathcal{G}_{1}, \mathcal{G}_{2}, \cdots, \mathcal{G}_{N}$ analíticas em zero e $\mathcal{G}_{1}(0)>0$, tal que se $G$ é analítica em zero da forma $G(y)=b y^{2 N}+O\left(y^{2 N+1}\right)$ com $b>0$, e satisfaz $l_{G}=l$, então a origem de (1.2) é um centro com função período $T$.

(ii) $O$ conjunto das funções $G$ analíticas em zero da forma $G(y)=b y^{2 N}+O\left(y^{2 N+1}\right)$ com $b>0$, satisfazendo $l_{G}=l$, está em correspondência biunívoca com o conjunto $\Sigma$ das involuções estritas analíticas. Mais concretamente, para cada $\sigma \in \Sigma$, a função correspondente $G$ é dada por $G(y)=\left[L^{-1}(y-\sigma(y))\right]^{2 N}$ onde

$L(y)=y \mathcal{G}_{1}\left(y^{2 N}\right)+y^{3} \mathcal{G}_{2}\left(y^{2 N}\right)+\cdots+y^{2 N-1} \mathcal{G}_{N}\left(y^{2 N}\right)$, com $\mathcal{G}_{1}, \mathcal{G}_{2}, \cdots, \mathcal{G}_{N}$ dadas em $(i)$.

Demonstração. (i) Pelo Lema 1.2.1, $l_{F}(h)=\sqrt{h} \mathcal{F}(h)$ com $\mathcal{F}(0)>0$, sendo assim

$l_{F}^{\prime}(h)=\frac{\frac{1}{2} \mathcal{F}(h)+h \mathcal{F}^{\prime}(h)}{\sqrt{h}}$. Seja $A(h)=\frac{1}{2} \mathcal{F}(h)+h \mathcal{F}^{\prime}(h), A(0)=\frac{1}{2} \mathcal{F}(0)>0$. Com isso e com $T$ satisfazendo as condições da Proposição 2.2.1, existem únicas funções analíticas $B_{1}, B_{2}, B_{3}, \cdots, B_{N}$, com $B_{1}(0)>0$ tais que

$$
T(h)=\int_{0}^{h} \frac{A(h-z)}{\sqrt{h-z}}\left(\frac{B_{1}(h)}{\sqrt[2 N]{h^{2 N-1}}}+\frac{B_{2}(h)}{\sqrt[2 N]{h^{2 N-3}}}+\cdots+\frac{B_{N}(h)}{\sqrt[2 N]{h}}\right) d z .
$$

Mostraremos que existe uma única maneira de escrevermos $B_{k}, k=1,2,3, \cdots, N$, como

$$
B_{k}(h)=\frac{(2 k-1) \mathcal{G}_{k}(h)}{2 N}+h \mathcal{G}_{k}^{\prime}(h)
$$

com $\mathcal{G}_{k}$ analítica em zero. 
Como $B_{k}$ é analítica em zero, $B_{k}(h)=\sum_{n=0}^{\infty} b_{n}^{k} h^{n}$ numa vizinhança $(-\epsilon, \epsilon)$ de zero, e $b_{0}^{1}=B_{1}(0)>0$.

Suponhamos que exista $\mathcal{G}_{k}$ analítica em zero escrita na forma $\mathcal{G}_{k}(h)=\sum_{n=0}^{\infty} c_{n}^{k} h^{n}$ numa vizinhança de zero que satisfaz (2.21). Então

$$
\begin{aligned}
B_{k}(h)=\sum_{n=0}^{\infty} b_{n}^{k} h^{n} & =\frac{2 k-1}{2 N} \sum_{n=0}^{\infty} c_{n}^{k} h^{n}+h \sum_{n=0}^{\infty}(n+1) c_{n+1}^{k} h^{n} \\
& =\frac{2 k-1}{2 N} \sum_{n=0}^{\infty} c_{n}^{k} h^{n}+\sum_{n=1}^{\infty} n c_{n}^{k} h^{n} \\
& =\frac{(2 k-1) c_{0}^{k}}{2 N}+\sum_{n=1}^{\infty}\left(\frac{2 k-1}{2 N}+n\right) c_{n}^{k} h^{n}
\end{aligned}
$$

ou seja, $b_{0}^{k}=\frac{(2 k-1) c_{0}^{k}}{2 N}$ e $b_{n}^{k}=\left(\frac{2 k-1}{2 N}+n\right) c_{n}^{k}, \quad n \geq 1$

Isto mostra a unicidade de $\mathcal{G}_{k}$ analítica em zero satisfazendo (2.21) numa certa vizinhança de zero.

Provemos a existência de $\mathcal{G}_{k}$ analítica em zero satisfazendo (2.21).

A candidata a $\mathcal{G}_{k}$ é dada por:

$$
\mathcal{G}_{k}(h)=\sum_{n=0}^{\infty} c_{n}^{k} h^{n}=c_{0}^{k}+\sum_{n=1}^{\infty} c_{n}^{k} h^{n}=\frac{2 N b_{0}^{k}}{2 k-1}+\sum_{n=1}^{\infty} \frac{2 N b_{n}^{k}}{2 k-1+2 N n} h^{n} .
$$

Como $\left|c_{n}^{k}\right|=\left|\frac{2 N b_{n}^{k}}{2 k-1+2 N n}\right| \leq\left|b_{n}^{k}\right|, \forall n \geq 1$, a série $\sum_{n=0}^{\infty} c_{n}^{k} h^{n}$ converge onde a série $\sum_{n=0}^{\infty} b_{n}^{k} h^{n}$ converge. Portanto $\mathcal{G}_{k}$ dada acima está bem definida e é analítica numa vizinhança da origem, e claramente satisfaz (2.21). Também $\mathcal{G}_{1}(0)=2 N b_{0}^{1}=2 N B_{1}(0)>0$.

Definamos $l(h)=\sqrt[2 N]{h}\left[\mathcal{G}_{1}(h)+\sqrt[N]{h} \mathcal{G}_{2}(h)+\cdots+\sqrt[N]{h^{N-1}} \mathcal{G}_{N}(h)\right]$.

Usando o Lema 2.2 .1 existe $G$ analítica em zero com mínimo na origem satisfazendo $l_{G}=l$. Então, pela observação 2.2 .2 , temos

$$
l_{G}^{\prime}(h)=\frac{B_{1}(h)}{\sqrt[2 N]{h^{2 N-1}}}+\frac{B_{2}(h)}{\sqrt[2 N]{h^{2 N-3}}}+\cdots+\frac{B_{N-1}(h)}{\sqrt[2 N]{h^{3}}}+\frac{B_{N}(h)}{\sqrt[2 N]{h}} .
$$

Usando $l_{F}^{\prime}(h)=\frac{\mathcal{F}(h)+h \mathcal{F}^{\prime}(h)}{2 \sqrt{h}}$ e $A(h)=\frac{1}{2} \mathcal{F}(h)+h \mathcal{F}^{\prime}(h)$, em (2.20), obtemos:

$$
T(h)=\int_{0}^{h} l_{F}^{\prime}(h-z) l_{G}^{\prime}(z) d z .
$$


Considere o sistema (1.2) com função de Hamilton $H(x, y)=F(x)+G(y)$. É claro que $H$ tem mínimo na origem e portanto a origem é um centro para (1.2).

Também é claro que nesse caso $T$ é a função período.

Com isso mostramos a existência de $l$ satisfazendo $(i)$.

Suponhamos que exista $l_{1}(h)=\sqrt[2 N]{h}\left[\widetilde{\mathcal{G}}_{1}(h)+\sqrt[N]{h} \widetilde{\mathcal{G}}_{2}(h)+\cdots+\sqrt[N]{h^{N-1}} \widetilde{\mathcal{G}}_{N}(h)\right], \widetilde{\mathcal{G}}_{1}(0)>0$ e $\widetilde{\mathcal{G}}_{k}, k=1,2,3, \cdots, N$, analíticas em zero, de modo que para toda $G$ analítica com mínimo na origem da forma $G(y)=b y^{2 N}+O\left(y^{2 N+1}\right)$ satisfazendo $l_{G}(h)=l_{1}(h)$ tenhamos em $(1.2)$ um centro de função período $T$. Então pela Observação 2.2.2, temos

$$
l_{G}^{\prime}(h)=\frac{\widetilde{B}_{1}(h)}{\sqrt[2 N]{h^{2 N-1}}}+\frac{\widetilde{B}_{2}(h)}{\sqrt[2 N]{h^{2 N-3}}}+\cdots+\frac{\widetilde{B}_{N}(h)}{\sqrt[2 N]{h}},
$$

com $\widetilde{B}_{1}, \widetilde{B}_{2}, \cdots, \widetilde{B}_{N}$ funções analíticas em zero, e $\widetilde{B}_{1}(0)=\frac{\widetilde{\mathcal{G}}_{1}(0)}{2 N}>0$. Pela Proposição 1.2 .2 ,

$$
T(h)=\int_{0}^{h} l_{F}^{\prime}(h-z) l_{G}^{\prime}(z) d z=\int_{0}^{h} \frac{A(h-z)}{\sqrt{h-z}}\left(\frac{\widetilde{B}_{1}(h)}{\sqrt[2 N]{h^{2 N-1}}}+\frac{\widetilde{B}_{2}(h)}{\sqrt[2 N]{h^{2 N-3}}}+\cdots+\frac{\widetilde{B}_{N}(h)}{\sqrt[2 N]{h}}\right) d z .
$$

Então, pela unicidade dada na Proposição 2.2.1,

$B_{k}=\widetilde{B}_{k}, k=1,2, \cdots, N$, e pela unicidade da representação dos $B_{k}, 1 \leq k \leq N$, em termos de $\mathcal{G}_{k}$, temos:

$$
\frac{(2 k-1) \mathcal{G}_{k}(h)}{2 N}+h \mathcal{G}_{k}^{\prime}(h)=\frac{(2 k-1) \widetilde{\mathcal{G}}_{k}(h)}{2 N}+h \widetilde{\mathcal{G}}_{k}^{\prime}(h)
$$

Disto decorre que $\mathcal{G}_{k}=\widetilde{\mathcal{G}_{k}}$, numa vizinhança da origem. De fato,

$$
\mathcal{G}_{k}(h)=\sum_{j=0}^{\infty} c_{j}^{k} h^{j} \Rightarrow \mathcal{G}_{k}^{\prime}(h)=\sum_{j=1}^{\infty} j c_{j}^{k} h^{j-1} \text { e } \widetilde{\mathcal{G}}_{k}(h)=\sum_{j=0}^{\infty} \widetilde{c}_{j}^{k} h^{j} \Rightarrow \widetilde{\mathcal{G}}_{k}^{\prime}(h)=\sum_{j=1}^{\infty} j \widetilde{c}_{j}^{k} h^{j-1} .
$$

Assim, obtemos

$$
\begin{aligned}
0 & =\frac{(2 k-1)}{2 N}\left(\mathcal{G}_{k}(h)-\widetilde{\mathcal{G}}_{k}(h)\right)+h\left(\mathcal{G}_{k}^{\prime}(h)-\widetilde{\mathcal{G}}_{k}^{\prime}(h)\right) \\
& =\frac{(2 k-1)}{2 N}\left(\sum_{j=0}^{\infty}\left(c_{j}^{k}-\widetilde{c}_{j}^{k}\right) h^{j}\right)+\sum_{j=1}^{\infty} j\left(c_{j}^{k}-\widetilde{c}_{j}^{k}\right) h^{j} \\
& =\frac{(2 k-1)}{2 N}\left(c_{0}^{k}-\widetilde{c}_{0}^{k}\right)+\sum_{k=1}^{\infty}\left(\frac{(2 k-1)}{2 N}+j\right)\left(c_{j}^{k}-\widetilde{c}_{j}^{k}\right) h^{j}
\end{aligned}
$$


numa vizinhança de zero. Logo, $c_{j}^{k}=\widetilde{c}_{j}^{k}, \forall j \geq 0$. Portanto, $l_{1}=l$ numa vizinhança da origem.

(ii) Seja $l(h)=\sqrt[2 N]{h}\left[\mathcal{G}_{1}(h)+\sqrt[N]{h} \mathcal{G}_{2}(h)+\cdots+\sqrt[N]{h^{N-1}} \mathcal{G}_{N}(h)\right]$, dada pelo ítem $(i)$.

Seja $\sigma$ uma involução estrita analítica. O Lema 2.2.4 garante que existe uma única função $G$ analítica em zero da forma, $G(y)=b y^{2 N}+O\left(y^{2 N+1}\right) \operatorname{com} b>0$ tal que $l_{G}(h)=G_{+}^{-1}(h)-G_{-}^{-1}(h)=l(h)$ e $\sigma(y)=y-\operatorname{sgn}(y) l(G(y))$. Pelo Lema 2.2 .7 $G(y)=\left[L^{-1}(y-\sigma(y))\right]^{2 N}$.

Por outro lado, dada $G(y)=b y^{2 N}+O\left(y^{2 N+1}\right)$ com $b>0$ analítica em zero, tal que $l_{G}=l$, segue do Lema 2.2 .2 que $\sigma(y)=y-\operatorname{sgn}(y) l(G(y))$ é uma involução estrita analítica. Novamente pelo Lema 2.2.7 $G(y)=\left[L^{-1}(y-\sigma(y))\right]^{2 N}$.

\subsubsection{Sistemas isócronos e comportamento da função período}

Nesta subseção obteremos algumas conseqüências dos resultados anteriores sobre o comportamento da função período.

Corolário 2.2.1 Suponhamos que em (1.2), F é analítica em zero com $l_{F}(h)=a \sqrt{h}$, com $a>0$ e $G(y)=b y^{2 N}+O\left(y^{2 N+1}\right)$, analítica em zero com $b>0$. Então a origem de (1.2) será um centro isócrono se e somente se $N=1$ e $l_{G}(h)=\beta \sqrt{h}, \beta>0$.

Demonstração. Se $N=1$, o Corolário 1.4 .1 garante que a origem é um centro se e só se $l_{G}(h)=\beta \sqrt{h}, \beta>0$.

Se $N \geq 2$, pelo Lema 2.2.6, $T(h)$ não é limitada perto de $h=0$, e assim não temos isocronismo.

O exemplo a seguir destaca uma propriedade que estudaremos no final deste capítulo. Exemplo: Seja $G(y)=\alpha y^{2 N}$, com $\alpha>0$ e $N \in \mathbb{N}$, e suponha que $l_{F}(h)=a \sqrt{h}, a>0$. Então $\sqrt[2 N]{h^{N-1}} T(h)$ será constante. Além disso, $l_{G}(h)=b \sqrt[2 N]{h}$, onde $b=\frac{2}{\sqrt{\alpha}}>0$. Assim,

De fato, numa vizinhança da origem, $G(y)=\alpha y^{2 N}=h$, se e só se $y= \pm \sqrt[2 N]{\frac{h}{\alpha}}=\frac{\sqrt[2 N]{h}}{\sqrt[2 N]{\alpha}}$.

$$
l_{G}(h)=G_{+}^{-1}(h)-G_{-}^{-1}(h)=\frac{\sqrt[2 N]{h}}{\sqrt[2 N]{\alpha}}-\left(-\frac{\sqrt[2 N]{h}}{\sqrt[2 N]{\alpha}}\right)=\frac{2}{\sqrt[2 N]{\alpha}} \sqrt[2 N]{h}
$$

e deste modo, $l_{G}(h)=b \sqrt[2 N]{h}, \quad b>0$.

$$
T(h)=\frac{d^{2}}{d h^{2}}\left(l_{F} * l_{G}\right)(h)=\frac{d^{2}}{d h^{2}}\left[\int_{0}^{h} l_{F}(h-z) l_{G}(z) d z\right]
$$


Fazendo a mudança de variáveis $z=h \operatorname{sen}^{2} \theta$, obtemos:

$$
\begin{gathered}
\int_{0}^{h} l_{F}(h-z) l_{G}(z) d z=2 \int_{0}^{\frac{\pi}{2}} a b \sqrt{h \cos ^{2} \theta} \sqrt[2 N]{h \operatorname{sen}^{2} \theta} h \operatorname{sen} \theta \cos \theta d \theta \\
=2 a b h^{\frac{3 N+1}{2 N}} \int_{0}^{\frac{\pi}{2}} \operatorname{sen}^{2} \theta \cos ^{2} \theta d \theta \\
T(h)=2 a b \frac{3 N+1}{2 N} \frac{N+1}{2 N} h^{\frac{-N+1}{2 N}} \int_{0}^{\frac{\pi}{2}} \operatorname{sen}^{2} \theta \cos ^{2} \theta d \theta
\end{gathered}
$$

Portanto, $\sqrt[2 N]{h^{N-1}} T(h)$ é constante.

Da proposição 1.5.1 temos algumas condições para assegurar a monotonicidade de $T$ quando $N=1$. Como $l_{F}$ e $l_{G}$ são funções crescentes em $(0, \bar{h})$, ocorre que $l_{F}^{\prime}$ e $l_{G}^{\prime}$ são funções positivas nesse intervalo, assim vemos que se $\psi_{F}$ e $\psi_{G}$ definidas na seção 1.2 por

$$
\psi_{F}(z)=\frac{l_{F}^{\prime}(z)+2 z l_{F}^{\prime \prime}(z)}{2} \text { e } \psi_{G}(z)=\frac{l_{G}^{\prime}(z)+2 z l_{G}^{\prime \prime}(z)}{2}
$$

são negativas em $(0, \bar{h})$ então $T$ será monótona decrescente nesse intervalo, pois pela Proposição 1.5.1 temos

$$
h T^{\prime}(h)=\left(\psi_{F} * l_{G}^{\prime}\right)(h)+\left(l_{F}^{\prime} * \psi_{G}\right)(h)
$$

Não devemos esperar que $\psi_{F}$ e $\psi_{G}$ sejam ambas positivas, quando $N>1$, em alguma vizinhança $\mathrm{V}$ de zero, pois nesse caso teríamos $T$ monótona crescente em $\mathrm{V}$ o que é impossível já que próximo da origem $T$ está próximo do infinito, se $N \geq 2$. Sendo assim próximo da origem nunca teremos $T$ crescente.

No entanto, podemos tentar estudar o comportamento de $\sqrt[2 N]{h^{N-1}} T(h)$.

Corolário 2.2.2 Seja $T$ a função período para o sistema (1.2) quando $F(x)=a x^{2}+O\left(x^{3}\right)$ e $G(y)=b y^{2 N}+O\left(y^{2 N+1}\right)$ são funções analíticas, $a>0$ e $b>0$. Suponhamos que $\sqrt[2 N]{h^{N-1}} T(h)$ seja constante e que $l_{F}(h)=c \sqrt{h}$ com $c>0$. Então $l_{G}(h)=d \sqrt[2 N]{h}$ com $d>0$.

Demonstração. Pelo Lema 2.2.6, $\sqrt[2 N]{h^{N-1}} T(h)$ é da forma

$$
\sqrt[2 N]{h^{N-1}} T(h)=T_{1}(h)+\sqrt[2 N]{h^{2}} T_{2}(h)+\sqrt[2 N]{h^{4}} T_{3}(h)+\cdots+\sqrt[2 N]{h^{2 N-2}} T_{N}(h), T_{1}(0)>0
$$

onde $T_{q}(h)=\sum_{n=0}^{\infty} t_{n}^{q} h^{n}$ numa vizinhança de 0 . 
Concluímos pelo Lema 2.2.5 que $\sqrt[2 N]{h^{N-1}} T(h)$ é constante, se e só se, $T_{1} \equiv T_{1}(0)>0$ e $T_{2} \equiv T_{3} \equiv \cdots \equiv T_{N}(h) \equiv 0$.

Pelo Lema 2.2.1

$$
l_{G}(h)=\sqrt[2 N]{h}\left(\mathcal{G}_{1}(h)+\sqrt[N]{h} \mathcal{G}_{2}(h)+\cdots+\sqrt[N]{h^{N-1}} \mathcal{G}_{N}(h)\right)
$$

onde $\mathcal{G}_{1}, \mathcal{G}_{2}, \cdots, \mathcal{G}_{N}$ são analíticas em zero $\operatorname{com} \mathcal{G}_{1}(0)>0$, e pela Observação 2.2 .2 ,

$$
B_{q}(h)=\frac{(2 q-1) \mathcal{G}_{q}(h)}{2 N}+h \mathcal{G}_{q}^{\prime}(h)=\sum_{n=0}^{\infty} b_{n}^{q} h^{n}, \quad q=1,2, \cdots, N
$$

satisfazem

$$
l_{G}^{\prime}(h)=\frac{B_{1}(h)}{\sqrt[2 N]{h^{2 N-1}}}+\frac{B_{2}(h)}{\sqrt[2 N]{h^{2 N-3}}}+\cdots+\frac{B_{N}(h)}{\sqrt[2 N]{h}}
$$

Por outro lado, $l_{F}^{\prime}(h)=\frac{c}{2 \sqrt{h}}=\frac{A(h)}{\sqrt{h}}$ onde $A(h)=\frac{c}{2}=\sum_{n=0}^{\infty} a_{n} h^{n} \operatorname{com} a_{0}=\frac{c}{2}>0 \mathrm{e}$ $a_{n}=0, n \geq 1$.

Agora de (2.17) na demonstração da Proposição 2.2.1, vemos que

$$
T_{q}(h)=\sum_{n=0}^{\infty}\left(\sum_{i+j=n} a_{i} b_{j}^{q} I_{i, j}^{q}\right) h^{n}=\sum_{n=0}^{\infty} \frac{c}{2} b_{n}^{q} I_{0, n}^{q} h^{n}
$$

ou seja, os coeficientes $t_{n}^{q}$ da série de Taylor de $T_{q}$ numa vizinhança de zero são dadas por

$$
t_{n}^{q}=\frac{c}{2} b_{n}^{q} I_{0, n}^{q}, \quad 1 \leq q \leq N
$$

Então, de $T_{1}(h) \equiv T_{1}(0)=t_{0}^{1}>0$ obtemos $t_{n}^{1}=0, n \geq 1$ e portanto $b_{0}^{1}>0$ e $b_{n}^{1}=0, n \geq 1$.

Assim obtemos

$$
b_{0}^{1}=B_{1}(h)=\frac{\mathcal{G}_{1}(h)}{2 N}+h \mathcal{G}_{1}^{\prime}(h)
$$

De $T_{q}(h) \equiv 0, q=2,3, \cdots, N$ obtemos de (2.24) que $b_{n}^{q}=0, n \geq 0, q=2,3, \cdots, N$. Assim,

$$
0 \equiv B_{q}(h)=\frac{(2 q-1) \mathcal{G}_{q}(h)}{2 N}+h \mathcal{G}_{q}^{\prime}(h), q=2,3, \cdots, N
$$


Como $\mathcal{G}_{q}$ é analítica podemos escrever $\mathcal{G}_{q}(h)=\sum_{n=0}^{\infty} c_{n}^{q} h^{n}$ numa vizinhança de zero, $1 \leq q \leq N$. Então, de (2.25) e (2.26) obtemos

$$
b_{0}^{1}=\frac{1}{2 N} \sum_{n=0}^{\infty} c_{n}^{1} h^{n}+h \sum_{n=0}^{\infty}(n+1) c_{n+1}^{1} h^{n}=\frac{c_{0}^{1}}{2 N}+\sum_{n=1}^{\infty}\left(\frac{1}{2 N}+n\right) c_{n}^{1} h^{n},
$$

e

$$
0=\frac{2 q-1}{2 N} \sum_{n=0}^{\infty} c_{n}^{q} h^{n}+h \sum_{n=0}^{\infty}(n+1) c_{n+1}^{q} h^{n}=\frac{(2 q-1) c_{0}^{q}}{2 N}+\sum_{n=1}^{\infty}\left(\frac{2 q-1}{2 N}+n\right) c_{n}^{q} h^{n}
$$

Logo $d=c_{0}^{1}=2 N b_{0}^{1}>0$ e $c_{n}^{1}=0, n \geq 1$, donde segue que $\mathcal{G}_{1}(h) \equiv c_{0}^{1}=d>0$, e para $2 \leq q \leq N$, temos $c_{n}^{q}=0, n \geq 0$, donde $\mathcal{G}_{q}(h) \equiv 0$.

Portanto de 2.22 segue que $l_{G}(h)=d \sqrt[2 N]{h} \operatorname{com} d>0$, como queríamos. 
Capítulo 3

\section{Uma generalização para sistemas hamiltonianos não clássicos.}

O nosso objetivo nesse capítulo é generalizar os resultados do capítulo 1 mudando principalmente a função $F$, que se apresentará sempre na forma $F(x)=a x^{4}+O\left(x^{4}\right), a>0$. Observe que, neste contexto, deixamos de trabalhar com sistemas hamiltonianos clássicos, em que a energia cinética $F(x)$ tem matriz hessiana não degenerada numa vizinhança de zero [Lim81] (como ocorria no caso em que $F(x)=a x^{2}+O\left(x^{3}\right)$, com $a>0$ ).

Os domínios de analiticidade $\Omega$ e $\widetilde{\Omega}$ das funções $F$ e $G$ aqui consideradas, bem como $\bar{x}_{-}, \bar{x}_{+}, \bar{y}_{-}, \bar{y}_{+}$e $\bar{h}$, satisfarão, a menos de menção explícita em contrário, as mesmas propriedades destacadas na seção 1.1 do capítulo 1 , e no início do capítulo 2.

\subsection{O caso em que $G$ tem mínimo não degenerado}

Seja $H(x, y)=\frac{x^{4}}{4}+\frac{y^{2}}{2}$, isto é, $F(x)=\frac{x^{4}}{4}$ e $G(y)=\frac{y^{2}}{2}$.

Então $G(y)=\frac{y^{2}}{2}=h \Leftrightarrow y= \pm \sqrt{2 h}$, de modo que

$$
l_{G}(h)=G_{+}^{-1}(h)-G_{-}^{-1}(h)=\sqrt{2 h}-(-\sqrt{2 h})=2 \sqrt{2 h}
$$

De modo análogo, $F(x)=\frac{x^{4}}{4}=h \Leftrightarrow x= \pm \sqrt[4]{4 h}$, donde

$$
l_{F}(h)=F_{+}^{-1}(h)-F_{-}^{-1}(h)=\sqrt[4]{4 h}-(-\sqrt[4]{4 h})=2 \sqrt[4]{4 h} .
$$

Assim obtemos $l_{G}^{\prime}(h)=\sqrt{\frac{2}{h}}$ e $l_{F}^{\prime}(h)=\frac{2}{\sqrt[4]{(4 h)^{3}}}$. Logo 
$T(h)=\left(l_{F}^{\prime} * l_{G}^{\prime}\right)(h)=\int_{0}^{h} l_{G}^{\prime}(z) l_{F}^{\prime}(h-z) d z=\int_{0}^{h} \frac{2 \sqrt{2}}{\sqrt{z} \sqrt[4]{(4(h-z))^{3}}} d z=\int_{0}^{h} \frac{1}{\sqrt{z} \sqrt[4]{(h-z})^{3}} d z$.

Fazendo a mudança de variáveis $z=h \cos ^{2} \theta$ obtemos,

$$
T(h)=2 \int_{0}^{\frac{\pi}{2}} \frac{1}{\sqrt{h \cos ^{2} \theta} \sqrt[4]{\left(h \operatorname{sen}^{2} \theta\right)^{3}}} h \operatorname{sen} \theta \cos \theta d \theta=\frac{2}{\sqrt[4]{h}} \int_{0}^{\frac{\pi}{2}} \frac{1}{\sqrt{\operatorname{sen} \theta}} d \theta
$$

Isto motivou-nos a estudar o caso não clássico em que $F(x)=a x^{4}+O\left(x^{5}\right)$.

Observação 3.1.1 Se $F(x)=a x^{4}+O\left(x^{5}\right)$, $a>0$ é analítica em $\Omega$ temos, pelo Fato 2.1.1, para $h>0$ suficientemente pequeno,

$$
l_{F}(h)=F_{+}^{-1}(h)-F_{-}^{-1}(h)=\sqrt[4]{h}\left(\sum_{n=0}^{\infty} b_{4 n+1} h^{n}+\sqrt{h} \sum_{n=0}^{\infty} b_{4 n+3} h^{n}\right),
$$

onde $b_{1}=\frac{2}{\sqrt[4]{a}}>0$. Também, pelo Fato 2.1.2 $(b), l_{F}^{\prime}(h)=\frac{A_{1}(h)}{\sqrt[4]{h^{3}}}+\frac{A_{2}(h)}{\sqrt[4]{h}}$, com $A_{1}$ e $A_{2}$ analíticas em zero e $A_{1}(0)>0$.

Observação 3.1.2 Usando o Lema 2.2.2 no caso em que $N=2$, se $F(x)=a x^{4}+O\left(x^{5}\right)$ analítica em $\Omega$ com $a>0$ temos, para $x \in\left(\bar{x}_{-}, \bar{x}_{+}\right)$, que

$$
\sigma(x)=x-\operatorname{sgn}(x) l_{F}(F(x))
$$

é uma involução estrita analítica tal que $F(x)=F(\sigma(x))$.

Observação 3.1.3 Do Lema 2.2.4 segue que se $l(h)=\sqrt[4]{h}(\delta(h)+\sqrt{h} \beta(h))$, onde $\delta$ e $\beta$ são analíticas em zero e $\delta(0)>0$, e $\sigma$ é uma involução estrita analítica, então existe uma única função $F$ analítica em zero da forma $F(x)=a x^{4}+O\left(x^{5}\right)$, com a $>0$, tal que $l(h)=F_{+}^{-1}(h)-F_{-}^{-1}(h)$, para $h>0$ suficientemente pequeno, e $\sigma(x)=x-\operatorname{sgn}(x) l(F(x))$.

Observação 3.1.4 Sejam $\left(a_{k}^{1}\right)_{k \in \mathbb{N}},\left(a_{k}^{2}\right)_{k \in \mathbb{N}},\left(t_{k}^{1}\right)_{k \in \mathbb{N}} e\left(t_{k}^{2}\right)_{k \in \mathbb{N}}$ seqüências reais com $a_{0}^{1}>0$ e $t_{0}^{1}>0$. Considere o sistema linear

$$
\begin{aligned}
& \text { (a) } \sum_{i+j=k} a_{i}^{1} b_{j} \widetilde{I}_{i, j}=t_{k}^{1}, k \in \mathbb{N} \\
& \text { (b) } \sum_{i+j=k} a_{i}^{2} b_{j} \widehat{I}_{i, j}=t_{k}^{2}, k \in \mathbb{N}
\end{aligned}
$$


onde

$$
\left\{\begin{array}{l}
\widetilde{I}_{i, j}=2 \int_{0}^{\frac{\pi}{2}} \operatorname{sen}^{\left(2 i-\frac{1}{2}\right)} \theta \cos ^{2 j} \theta d \theta=\beta\left(i+\frac{1}{4}, j+\frac{1}{2}\right)=\frac{\Gamma\left(i+\frac{1}{4}\right) \Gamma\left(j+\frac{1}{2}\right)}{\Gamma\left(i+j+\frac{3}{4}\right)} \\
\widehat{I}_{i, j}=2 \int_{0}^{\frac{\pi}{2}} \operatorname{sen}^{\left(2 i+\frac{1}{2}\right)} \theta \cos ^{2 j} \theta d \theta=\beta\left(i+\frac{3}{4}, j+\frac{1}{2}\right)=\frac{\Gamma\left(i+\frac{3}{4}\right) \Gamma\left(j+\frac{1}{2}\right)}{\Gamma\left(i+j+\frac{5}{4}\right)}
\end{array}\right.
$$

Note que $\widetilde{I}_{i, j}=I_{j, i}^{1}$ e $\widehat{I}_{i, j}=I_{j, i}^{2}$ onde $I_{j, i}^{q}, q=1,2$, são dadas pela definição A.0.2 com $N=2$. Considerando apenas $k=0,1, \cdots, n,(a)$ pode ser escrito como:

$$
\left[\begin{array}{ccccc}
a_{0}^{1} \widetilde{I}_{0, n} & a_{1}^{1} \widetilde{I}_{1, n-1} & a_{2}^{1} \widetilde{I}_{2, n-2} & \ldots & a_{n}^{1} \widetilde{I}_{n, 0} \\
0 & a_{0}^{1} \widetilde{I}_{0, n-1} & a_{1}^{1} \widetilde{I}_{1, n-2} & \ldots & a_{n-1}^{1} \widetilde{I}_{n-1,0} \\
0 & 0 & a_{0}^{1} \widetilde{I}_{0, n-2} & \ldots & a_{n-2}^{1} \widetilde{I}_{n-2,0} \\
\vdots & \vdots & \vdots & \ddots & \vdots \\
0 & 0 & 0 & \ldots & a_{0}^{1} \widetilde{I}_{0,0}
\end{array}\right]\left[\begin{array}{c}
b_{n} \\
b_{n-1} \\
b_{n-2} \\
\vdots \\
b_{0}
\end{array}\right]=\left[\begin{array}{c}
t_{n}^{1} \\
t_{n-1}^{1} \\
t_{n-2}^{!} \\
\vdots \\
t_{0}^{1}
\end{array}\right]
$$

Podemos ver que a matriz

$$
\left[\mathbf{J}_{\mathbf{n}}^{\mathbf{1}}\right]=\left[\begin{array}{ccccc}
a_{0}^{1} \widetilde{I}_{0, n} & a_{1}^{1} \widetilde{I}_{1, n-1} & a_{2}^{1} \widetilde{I}_{2, n-2} & \ldots & a_{n}^{1} \widetilde{I}_{n, 0} \\
0 & a_{0}^{1} \widetilde{I}_{0, n-1} & a_{1}^{1} \widetilde{I}_{1, n-2} & \ldots & a_{n-1}^{1} \widetilde{I}_{n-1,0} \\
0 & 0 & a_{0}^{1} \widetilde{I}_{0, n-2} & \ldots & a_{n-2}^{1} \widetilde{I}_{n-2,0} \\
\vdots & \vdots & \vdots & \ddots & \vdots \\
0 & 0 & 0 & \ldots & a_{0}^{1} \widetilde{I}_{0,0}
\end{array}\right]
$$

é inversível e que $\left[\mathbf{B}_{\mathbf{n}}\right]=\left[\mathbf{J}_{\mathbf{n}}^{\mathbf{1}}\right]^{-\mathbf{1}}\left[\mathrm{t}_{\mathbf{n}}^{\mathbf{1}}\right]$, onde

$$
\left[\mathbf{B}_{\mathbf{n}}\right]=\left[\begin{array}{c}
b_{n} \\
b_{n-1} \\
b_{n-2} \\
\vdots \\
b_{0}
\end{array}\right] e\left[\mathbf{t}_{\mathbf{n}}^{\mathbf{1}}\right]=\left[\begin{array}{c}
t_{n}^{1} \\
t_{n-1}^{1} \\
t_{n-2}^{1} \\
\vdots \\
t_{0}^{1}
\end{array}\right]
$$

Do mesmo modo, considerando apenas $k=0,1, \cdots, n,(b)$ pode ser reescrito como, $\left[\mathbf{J}_{\mathbf{n}}^{2}\right]\left[\mathbf{B}_{\mathbf{n}}\right]=\left[\mathbf{t}_{\mathbf{n}}^{2}\right]$, onde

$$
\left[\mathbf{J}_{\mathbf{n}}^{2}\right]=\left[\begin{array}{ccccc}
a_{0}^{2} \widehat{I}_{0, n} & a_{1}^{2} \widehat{I}_{1, n-1} & a_{2}^{2} \widehat{I}_{2, n-2} & \ldots & a_{n}^{2} \widehat{I}_{n, 0} \\
0 & a_{0}^{2} \widehat{I}_{0, n-1} & a_{1}^{2} \widehat{I}_{1, n-2} & \ldots & a_{n-1}^{2} \widehat{I}_{n-1,0} \\
0 & 0 & a_{0}^{2} \widehat{I}_{0, n-2} & \ldots & a_{n-2}^{2} \widehat{I}_{n-2,0} \\
\vdots & \vdots & \vdots & \ddots & \vdots \\
0 & 0 & 0 & \ldots & a_{0}^{2} \widehat{I}_{0,0}
\end{array}\right] e\left[\mathbf{t}_{\mathbf{n}}^{2}\right]=\left[\begin{array}{c}
t_{n}^{2} \\
t_{n-1}^{2} \\
t_{n-2}^{2} \\
\vdots \\
t_{0}^{2}
\end{array}\right]
$$


Assim, se olharmos para $(a)$ e (b) com $0 \leq k \leq n$ como um sistema de $2 n+2$ equações em $\left[\mathbf{B}_{\mathbf{n}}\right]$, para termos existência e unicidade de solução é necessário e suficiente que a relação $\left[\mathbf{J}_{\mathbf{n}}^{2}\right]\left[\mathbf{J}_{\mathbf{n}}^{1}\right]^{-\mathbf{1}}\left[\mathbf{t}_{\mathbf{n}}^{\mathbf{1}}\right]=\left[\mathrm{t}_{\mathbf{n}}^{2}\right]$ seja satisfeita.

Na Proposição a seguir, $\left[\mathbf{J}_{\mathbf{n}}^{1}\right]$ e $\left[\mathbf{J}_{\mathbf{n}}^{2}\right]$ serão as matrizes definidas na Observação 3.1.4.

Proposição 3.1.1 Sejam $A_{1}(h)=\sum_{n=0}^{\infty} a_{n}^{1} h^{n}$ e $A_{2}(h)=\sum_{n=0}^{\infty} a_{n}^{2} h^{n}, h \in[0, R)$, com $A_{1}(0)=a_{0}^{1}>0$ e seja $T(h)=\frac{1}{\sqrt[4]{h}}\left(T_{1}(h)+\sqrt{h} T_{2}(h)\right)$, onde $T_{1}(h)=\sum_{n=0}^{\infty} t_{n}^{1} h^{n} e$ $T_{2}(h)=\sum_{n=0}^{\infty} t_{n}^{2} h^{n}, h \in[0, R)$, com $T_{1}(0)=t_{\mathbf{0}}^{1}>0$, tais que $\left[\mathbf{J}_{\mathbf{n}}^{2}\right]\left[\mathbf{J}_{\mathbf{n}}^{\mathbf{1}}\right]^{-1}\left[\mathbf{t}_{\mathbf{n}}^{\mathbf{1}}\right]=\left[\mathbf{t}_{\mathbf{n}}^{2}\right], \forall n \in \mathbb{N}$.

Então a equação integral

$$
T(h)=\int_{0}^{h}\left(\frac{A_{1}(h-z)}{\sqrt[4]{(h-z)^{3}}}+\frac{A_{2}(h-z)}{\sqrt[4]{h-z}}\right) \cdot \frac{B(z)}{\sqrt{z}} d z
$$

tem uma única solução analítica $B$ tal que $B(0)>0$.

Observação: Como nos capítulos anteriores, a unicidade no enunciado anterior significa que se $B:[0, r) \rightarrow \mathbb{R}$ e $B_{1}:\left[0, r_{1}\right) \rightarrow \mathbb{R}$ são duas soluções analíticas de (3.3), então $B=B_{1}$ em $[0, r) \cap\left[0, r_{1}\right)$.

Demonstração.

Comecemos provando a unicidade de solução. Seja $B(z)$ uma solução analítica em $[0, r), 0<r \leq R$, e suponhamos que $B(z)=\sum_{n=0}^{\infty} b_{n} z^{n}$ em $\left[0, \epsilon_{0}\right)$, para algum $\epsilon_{0}>0$, $\epsilon_{0} \leq r$.

Por meio da mudança de variáveis $z=h \cos ^{2} \theta$ em (3.3) e usando o Lema 1.2.5 podemos 
proceder como na demonstração do ítem $(C)$ do Fato 2.1 .2 e obter

$$
\begin{aligned}
& \frac{1}{\sqrt[4]{h}}\left[\sum_{n=0}^{\infty} t_{n}^{1} h^{n}+\sqrt{h} \sum_{n=0}^{\infty} t_{n}^{2} h^{n}\right]=T(h) \\
= & \frac{2}{\sqrt[4]{h}}\left[\int_{0}^{\frac{\pi}{2}} A_{1}\left(h \operatorname{sen}^{2} \theta\right) \frac{B\left(h \cos ^{2} \theta\right)}{\sqrt{\operatorname{sen} \theta}} d \theta+\sqrt{h} \int_{0}^{\frac{\pi}{2}} \sqrt{\operatorname{sen} \theta} A_{2}\left(h \operatorname{sen}^{2} \theta\right) B\left(h \cos ^{2} \theta\right) d \theta\right] \\
= & \frac{2}{\sqrt[4]{h}} \int_{0}^{\frac{\pi}{2}} \sum_{n=0}^{\infty} a_{n}^{1}\left(h \operatorname{sen}^{2} \theta\right)^{n} \sum_{n=0}^{\infty} \frac{b_{n}\left(h \cos ^{2} \theta\right)^{n}}{\sqrt{\operatorname{sen} \theta}} d \theta \\
+ & 2 \sqrt{h} \int_{0}^{\frac{\pi}{2}} \sqrt{\operatorname{sen} \theta} \sum_{n=0}^{\infty} a_{n}^{2}\left(h \operatorname{sen}^{2} \theta\right)^{n} \sum_{n=0}^{\infty} b_{n}\left(h \cos ^{2} \theta\right)^{n} d \theta \\
= & \frac{2}{\sqrt[4]{h}} \int_{0}^{\frac{\pi}{2}} \sum_{n=0}^{\infty}\left(\sum_{i+j=n} \frac{\left(a_{i}^{1} b_{j} \operatorname{sen}^{2 i} \theta \cos ^{2 j} \theta\right)}{\sqrt{\operatorname{sen} \theta}} h^{n}\right) d \theta \\
+ & 2 \sqrt{h} \int_{0}^{\frac{\pi}{2}} \sum_{n=0}^{\infty}\left(\sum_{i+j=n}\left(a_{i}^{2} b_{j} \operatorname{sen}^{2 i} \theta \cos ^{2 j} \theta \sqrt{\operatorname{sen} \theta}\right) h^{n}\right) d \theta \\
= & \frac{2}{\sqrt[4]{h}} \sum_{n=0}^{\infty}\left(\sum_{i+j=n} a_{i}^{1} b_{j}\left(2 \int_{0}^{\frac{\pi}{2}} \operatorname{sen}^{\left(2 i-\frac{1}{2}\right)} \theta \cos ^{2 j} \theta d \theta\right)\right) h^{n} \\
+ & \sqrt{h} \sum_{n=0}^{\infty}\left(\sum_{i+j=n} a_{i}^{2} b_{j}\left(2 \int_{0}^{\frac{\pi}{2}} \operatorname{sen}^{\left(2 i+\frac{1}{2}\right)} \theta \cos ^{2 j} \theta d \theta\right)\right) h^{n} \\
= & \frac{2}{\sqrt[4]{h}} \sum_{n=0}^{\infty}\left(\sum_{i+j=n} a_{i}^{1} b_{j} \tilde{I}_{i, j}\right) h^{n}+\sqrt{h} \sum_{n=0}^{\infty}\left(\sum_{i+j=n} a_{i}^{2} b_{j} \widehat{I}_{i, j}\right) h^{n}
\end{aligned}
$$

onde, $\widetilde{I}_{i, j}=2 \int_{0}^{\frac{\pi}{2}} \operatorname{sen}^{\left(2 i-\frac{1}{2}\right)} \theta \cos ^{2 j} \theta d \theta$ e $\widehat{I}_{i, j}=2 \int_{0}^{\frac{\pi}{2}} \operatorname{sen}^{\left(2 i+\frac{1}{2}\right)} \theta \cos ^{2 j} \theta d \theta$ são dados por (3.2).

Assim obtemos:

$$
\underbrace{t_{n}^{1}=\sum_{i+j=n} a_{i}^{1} b_{j} \widetilde{I}_{i, j}}_{(a)} \text { e } \underbrace{t_{n}^{2}=\sum_{i+j=n} a_{i}^{2} b_{j} \widehat{I}_{i, j}}_{(b)}, k \in \mathbb{N}
$$

O sistema ( $a$ ) com $0 \leq k \leq n$ do qual $\left(b_{1}, b_{2}, \cdots, b_{n}\right)$ é solução possível e determinado (ver Observação 3.1.4). Isto prova a unicidade local de $B$.

A unicidade global segue do princípio do prolongamento analítico.

Para mostrar a existência de solução $B$ definida numa vizinhança de zero basta mos- 
trarmos que a série $\sum_{n=0}^{\infty} b_{n} h^{n}$, onde os coeficientes $b_{n}$ são determinados por $(a)$, converge em alguma vizinhança de zero. Então, da hipótese $\left[\mathbf{J}_{\mathbf{n}}^{\mathbf{2}}\right]\left[\mathbf{J}_{\mathbf{n}}^{\mathbf{1}}\right]^{-\mathbf{1}}\left[\mathbf{t}_{\mathbf{n}}^{\mathbf{1}}\right]=\left[\mathbf{t}_{\mathbf{n}}^{\mathbf{2}}\right], n \in \mathbb{N}$, seguirá imediatamente que tais $b_{j}$ 's satisfazem também $(b)$, e do Lema 1.2.5 decorrerá que as igualdades em (3.4) serão satisfeitas.

Podemos supor sem perda de generalidade $a_{0}=1$.

Procedendo como na Proposição 1.2.3 tomemos $C>0$ de modo que

$$
\left|a_{n}^{1}\right| \leq C^{n} \text { e }\left|t_{n}^{1}\right| \leq \frac{\Gamma\left(\frac{1}{4}\right)}{\Gamma\left(\frac{3}{4}\right)} \frac{C^{n+1}}{3}, \forall n \in \mathbb{N}
$$

Afirmamos que

$$
\left|b_{k}\right| \leq(k+1) \frac{C^{k+1}}{\sqrt{\pi}}
$$

Provaremos esta afirmação por indução em $k$.

Para $k=0$ temos, por $(a), b_{0}=\frac{t_{0}^{1}}{\widetilde{I}_{0,0}}$, portanto,

$$
\left|b_{0}\right|=\frac{\left|t_{0}^{1}\right|}{\left|\widetilde{I}_{0,0}\right|} \leq \frac{\frac{\Gamma\left(\frac{1}{4}\right)}{\Gamma\left(\frac{3}{4}\right)} \frac{C^{0+1}}{3}}{\frac{\Gamma\left(\frac{1}{4}\right)}{\Gamma\left(\frac{3}{4}\right)} \sqrt{\pi}}=\frac{C}{3 \sqrt{\pi}}<C
$$

pois $\widetilde{I}_{0,0}=\frac{\Gamma\left(\frac{1}{4}\right)}{\Gamma\left(\frac{3}{4}\right)} \sqrt{\pi}$ (Fato A.0.21).

Para $k=1$ temos, por $(a), b_{1}=\frac{t_{1}^{1}-a_{1}^{1} b_{0} \widetilde{I}_{1,0}}{\widetilde{I}_{0,1}}, \operatorname{logo}$

$$
\left|b_{1}\right| \leq \frac{\left|t_{1}^{1}\right|+\left|a_{1}^{1}\right|\left|b_{0}\right| \widetilde{I}_{1,0}}{\widetilde{I}_{0,1}} \leq \frac{\frac{\Gamma\left(\frac{1}{4}\right)}{\Gamma\left(\frac{3}{4}\right)} \frac{C^{2}}{3}}{\frac{\Gamma\left(\frac{1}{4}\right) \frac{1}{2} \sqrt{\pi}}{\frac{3}{4} \Gamma\left(\frac{3}{4}\right)}}+\frac{C \frac{C}{3 \sqrt{\pi}} \widetilde{I}_{1,0}}{\widetilde{I}_{0,1}}=\frac{C^{2}}{2 \sqrt{\pi}}+\frac{C^{2}}{3 \sqrt{\pi}}<2 C^{2}
$$

pois $\frac{\widetilde{I}_{1,0}}{\widetilde{I}_{0,1}} \leq 1$ (Fato A.0.24), e $\widetilde{I}_{0,1}=\frac{\Gamma\left(\frac{1}{4}\right) \frac{1}{2} \sqrt{\pi}}{\frac{3}{4} \Gamma\left(\frac{3}{4}\right)}$ (Fato A.0.22).

Suponhamos a desigualdade 3.5 válida para $k=0,1, \cdots, n-1$ onde $n \geq 2$. Então por (a) temos:

$$
b_{n}=\frac{t_{n}^{1}-a_{i}^{1} b_{n-1} \widetilde{I}_{1, n-1}-a_{2}^{1} b_{n-2} \widetilde{I}_{2, n-2}-\cdots-a_{n} b_{0} \widetilde{I}_{n, 0}}{\widetilde{I}_{0, n}}
$$


portanto,

$$
\begin{aligned}
\left|b_{n}\right| & \leq \frac{\left|t_{n}^{1}\right|+\left|a_{1}^{1}\right|\left|b_{n-1}\right| \widetilde{I}_{1, n-1}+\left|a_{2}^{1}\right|\left|b_{n-2}\right| \widetilde{I}_{2, n-2}+\cdots+\left|a_{n}^{1}\right|\left|b_{0}\right| \widetilde{I}_{n, 0}}{\widetilde{I}_{0, n}} \\
& \leq \frac{\Gamma\left(\frac{1}{4}\right)}{\Gamma\left(\frac{3}{4}\right)} \frac{C^{n+1}}{3}+C n \frac{C^{n}}{\sqrt{\pi}} \widetilde{I}_{1, n-1}+C^{2}(n-1) \frac{C^{n-1}}{\sqrt{\pi}} \widetilde{I}_{2, n-2}+\cdots+C^{n} \frac{C}{\sqrt{\pi}} \widetilde{I}_{n, 0} \\
& =C^{n+1}\left[\frac{\Gamma\left(\frac{1}{4}\right)}{3 \Gamma\left(\frac{3}{4}\right) \widetilde{I}_{0, n}}+\frac{\widetilde{I}_{n, 0}}{\widetilde{I}_{0, n} \sqrt{\pi}}+\frac{n \widetilde{I}_{1, n-1}+(n-1) \widetilde{I}_{2, n-2}+\cdots+2 \widetilde{I}_{n-1,1}}{\sqrt{\pi} \widetilde{I}_{0, n}}\right]
\end{aligned}
$$

Agora do Fato, A.0.23 para $1 \leq j \leq n-1$ segue que $\widetilde{I}_{j, n-j} \leq \widetilde{I}_{1, n-1}$. Pelos Fatos A.0.24, A.0.25 e A.0.26 temos respectivamente $\frac{\widetilde{I}_{n, 0}}{\widetilde{I}_{0, n}} \leq 1, \frac{(n+2)(n-1)}{2} \frac{\widetilde{I}_{1, n-1}}{\widetilde{I}_{0, n}} \leq \frac{n}{4} \mathrm{e}$ $\frac{\Gamma\left(\frac{1}{4}\right) \sqrt{\pi}}{3 \Gamma\left(\frac{3}{4}\right) \widetilde{I}_{0, n}}<\frac{3 n}{4}$ par todo $n \geq 2$, e portanto,

$$
\begin{aligned}
\left|b_{n}\right| & \leq C^{n+1}\left[\frac{\Gamma\left(\frac{1}{4}\right)}{3 \Gamma\left(\frac{3}{4}\right) \widetilde{I}_{0, n}}+\frac{\widetilde{I}_{n, 0}}{\widetilde{I}_{0, n} \sqrt{\pi}}+\frac{(n+(n-1)+\cdots+2) \widetilde{I}_{1, n-1}}{\sqrt{\pi} \widetilde{I}_{0, n}}\right] \\
& =C^{n+1}\left[\frac{\Gamma\left(\frac{1}{4}\right)}{3 \Gamma\left(\frac{3}{4}\right) \widetilde{I}_{0, n}}+\frac{\widetilde{I}_{n, 0}}{\widetilde{I}_{0, n} \sqrt{\pi}}+\frac{\frac{(n+2)(n-1) \widetilde{I}_{1, n-1}}{2}}{\sqrt{\pi} \widetilde{I}_{0, n}}\right] \leq(n+1) \frac{C^{n+1}}{\sqrt{\pi}} .
\end{aligned}
$$

Portanto $\left|b_{k}\right| \leq(k+1) \frac{C^{k+1}}{\sqrt{\pi}}, \forall k \in \mathbb{N}$.

Teorema 3.1.1 Seja $F(x)=a x^{4}+O\left(x^{5}\right) \quad$ analítica em zero, $a>0, \quad e$ seja $l_{F}(h)=F_{+}^{-1}(h)-F_{-}^{-1}(h), h \in[0, \bar{h}]$. Considere $A_{1}(h)=\sum_{n=0}^{\infty} a_{n}^{1} h^{n}$ e $A_{2}(h)=\sum_{n=0}^{\infty} a_{n}^{2} h^{n}$ dadas na Observação 3.1.1. Seja também $T(h)=\frac{1}{\sqrt[4]{h}}\left[T_{1}(h)+\sqrt{h} T_{2}(h)\right]$, com $T_{1}$ e $T_{2}$ satisfazendo as hipóteses da Proposição 3.1.1. Então as seguintes afirmações são verdadeiras:

(i) Existe uma única $l(h)=\sqrt{h} \mathcal{L}(h)$ com $\mathcal{L}$ analítica em zero e $\mathcal{L}(0)>0$ tal que se $G$ é analítica em zero da forma $G(y)=b y^{2}+O\left(y^{3}\right)$ com $b>0$ e satisfaz $l_{G} \equiv l$, então a origem de (1.2) é um centro com função período $T$.

(ii) Além disso, o conjunto das funçôes $G$ analíticas em zero da forma $G(y)=b y^{2}+O\left(y^{3}\right)$ com $b>0$ que satisfazem $l_{G} \equiv l$ está em correspondência biunívoca com o conjunto $\Sigma$ das involuções estritas analíticas. Mais concretamente, para cada $\sigma \in \Sigma$ a função correspondente $G$ é dada por $G(y)=\left[L^{-1}(y-\sigma(y))\right]^{2}$ onde $L(y)=y \mathcal{L}\left(y^{2}\right)$ com $\mathcal{L}$ dada em (i). 
Demonstração. A demonstração é análoga à do Teorema 1.3.1, para isto basta usar a Observação 3.1.1 em vez do Lema 1.2.1 e a Proposição 3.1.1 em vez da Proposição 1.2.3.

De fato, a Observação 3.1 .1 nos garante que $l_{F}^{\prime}(h)=\frac{A_{1}(h)}{\sqrt[4]{h^{3}}}+\frac{A_{2}(h)}{\sqrt[4]{h}}$, com $A_{1}$ e $A_{2}$ analíticas em zero e $A_{1}(0)>0$, e, das hipóteses sobre $T$, a Proposição 3.1 .1 nos garante que a equação integral

$$
T(h)=\int_{0}^{h}\left(\frac{A_{1}(h-z)}{\sqrt[4]{(h-z)^{3}}}+\frac{A_{2}(h-z)}{\sqrt[4]{h-z}}\right) \frac{B(z)}{\sqrt{z}} d z
$$

possui uma única solução analítica $B$ tal que $B(0)>0$.

A partir deste ponto a demonstração é exatamente igual à do Teorema 1.3.1.

\subsection{O caso em que $G$ tem mínimo degenerado}

Trataremos aqui apenas o caso em que $G$ é da forma $G(y)=b y^{4}+O\left(y^{5}\right) \operatorname{com} b>0$. O tratamento do caso geral $G(y)=b y^{2 N}+O\left(y^{2 N+1}\right)$, com $N \geq 3$, parece-nos ser análogo, apenas envolvendo muito mais contas.

Lema 3.2.1 Sejam $F(x)=a x^{4}+O\left(x^{5}\right)$ com $a>0$ e $G(y)=b y^{4}+O\left(y^{5}\right) \operatorname{com} b>0$ analíticas em zero. Então a função período associada a (1.2) é da forma:

$$
T(h)=\frac{1}{\sqrt{h}}\left(T_{1}(h)+\sqrt{h} T_{2}(h)\right)
$$

com $T_{1}$ e $T_{2}$ analíticas em zero e $T_{1}(0)>0$.

Demonstração. Pelo Lema 2.1.2

$$
l_{F}^{\prime}(h)=\frac{A_{1}(h)}{\sqrt[4]{h^{3}}}+\frac{A_{2}(h)}{\sqrt[4]{h}} \text { e } l_{G}^{\prime}(h)=\frac{B_{1}(h)}{\sqrt[4]{h^{3}}}+\frac{B_{2}(h)}{\sqrt[4]{h}},
$$

onde $A_{1}, A_{2}, B_{1}$ e $B_{2}$ são analíticas em zero e $A_{1}(0)>0, B_{1}(0)>0$. Logo, pela Proposição 1.2.2, temos:

$T(h)=\left(l_{F}^{\prime} * l_{G}^{\prime}\right)(h)=\int_{0}^{h} l_{F}^{\prime}(z) l_{G}^{\prime}(h-z) d z=\int_{0}^{h}\left(\frac{A_{1}(h-z)}{\sqrt[4]{(h-z)^{3}}}+\frac{A_{2}(h-z)}{\sqrt[4]{h-z}}\right)\left(\frac{B_{1}(z)}{\sqrt[4]{z^{3}}}+\frac{B_{2}(z)}{\sqrt[4]{z}}\right) d z$

Sejam $A_{1}(h)=\sum_{n=0}^{\infty} a_{n}^{1} h^{n}, A_{2}(h)=\sum_{n=0}^{\infty} a_{n}^{2} h^{n}, B_{1}(h)=\sum_{n=0}^{\infty} b_{n}^{1} h^{n}$ e $B_{2}(h)=\sum_{n=0}^{\infty} b_{n}^{2} h^{n}$ as expansões em série de Taylor de $A_{1}, A_{2}, B_{1}$, e $B_{2}$ em $(-\epsilon, \epsilon)$. Façamos a mudança de 
variáveis $z=h \cos ^{2} \theta$. Então, usando o Lema 1.2.5, temos

$$
\begin{aligned}
& T(h)= \\
& =2 \int_{0}^{\frac{\pi}{2}}\left(\frac{A_{1}\left(h \operatorname{sen}^{2} \theta\right)}{\sqrt[4]{\left(h \operatorname{sen}^{2} \theta\right)^{3}}}+\frac{A_{2}\left(h \operatorname{sen}^{2} \theta\right)}{\sqrt[4]{h \operatorname{sen}^{2} \theta}}\right)\left(\frac{B_{1}\left(h \cos ^{2} \theta\right)}{\sqrt[4]{\left(h \cos ^{2} \theta\right)^{3}}}+\frac{B_{2}\left(h \cos ^{2} \theta\right)}{\sqrt[4]{h \cos ^{2} \theta}}\right) h \operatorname{sen} \theta \cos \theta d \theta \\
& =2 \int_{0}^{\frac{\pi}{2}}\left(\frac{A_{1}\left(h \operatorname{sen}^{2} \theta\right)}{\sqrt{h} \operatorname{sen} \theta}+A_{2}\left(h \operatorname{sen}^{2} \theta\right)\right)\left(\frac{B_{1}\left(h \cos ^{2} \theta\right)}{\sqrt{h} \cos \theta}+B_{2}\left(h \cos ^{2} \theta\right)\right) \sqrt{h \operatorname{sen} \theta \cos \theta} d \theta \\
& =\frac{2}{\sqrt{h}}\left[\int_{0}^{\frac{\pi}{2}}\left(\frac{A_{1}\left(h \operatorname{sen}^{2} \theta\right) B_{1}\left(h \cos ^{2} \theta\right)}{\sqrt{\operatorname{sen} \theta \cos \theta}}+h \sqrt{\operatorname{sen} \theta \cos \theta} A_{2}\left(h \operatorname{sen}^{2} \theta\right) B_{2}\left(h \cos ^{2} \theta\right)\right) d \theta\right. \\
& \left.+\sqrt{h} \int_{0}^{\frac{\pi}{2}}\left(\frac{\sqrt{\operatorname{sen} \theta} A_{2}\left(h \operatorname{sen}^{2} \theta\right) B_{1}\left(h \cos ^{2} \theta\right)}{\sqrt{\cos \theta}}+\frac{\sqrt{\cos \theta} A_{1}\left(h \operatorname{sen}^{2} \theta\right) B_{2}\left(h \cos ^{2} \theta\right)}{\sqrt{\operatorname{sen} \theta}}\right) d \theta\right] \\
& =\frac{2}{\sqrt{h}}\left[\int_{0}^{\frac{\pi}{2}} \sum_{k=0}^{\infty}\left[\sum_{i+j=k}\left(\frac{a_{i}^{1} b_{j}^{1} \operatorname{sen}^{2 i} \theta \cos ^{2 j} \theta}{\sqrt{\operatorname{sen} \theta \cos \theta}}+h \sqrt{\operatorname{sen} \theta \cos \theta} a_{i}^{2} b_{j}^{2} \operatorname{sen}^{2 i} \theta \cos ^{2 j} \theta\right)\right] h^{k} d \theta\right. \\
& \left.+\sqrt{h} \int_{0}^{\frac{\pi}{2}} \sum_{k=0}^{\infty}\left[\sum_{i+j=k}\left(\frac{\sqrt{\operatorname{sen} \theta} a_{i}^{2} b_{j}^{1} \operatorname{sen}^{2 i} \theta \cos ^{2 j} \theta}{\sqrt{\cos \theta}}+\frac{\sqrt{\cos \theta} a_{i}^{1} b_{j}^{2} \operatorname{sen}^{2 i} \theta \cos ^{2 j} \theta}{\sqrt{\operatorname{sen} \theta}}\right)\right] h^{k} d \theta\right] \\
& =\frac{2}{\sqrt{h}}\left[\int_{0}^{\frac{\pi}{2}} \sum_{k=0}^{\infty}\left(\sum_{i+j=k} a_{i}^{1} b_{j}^{1} \operatorname{sen}^{\left(2 i-\frac{1}{2}\right)} \theta \cos ^{\left(2 j-\frac{1}{2}\right)} \theta\right) h^{k} d \theta\right. \\
& +h \int_{0}^{\frac{\pi}{2}} \sum_{k=0}^{\infty}\left(\sum_{i+j=k} a_{i}^{2} b_{j}^{2} \operatorname{sen}^{\left(2 i+\frac{1}{2}\right)} \theta \cos ^{\left(2 j+\frac{1}{2}\right)} \theta\right) h^{k} d \theta \\
& +\sqrt{h}\left(\int_{0}^{\frac{\pi}{2}} \sum_{k=0}^{\infty}\left(\sum_{i+j=k} a_{i}^{2} b_{j}^{1} \operatorname{sen}^{\left(2 i+\frac{1}{2}\right)} \theta \cos ^{\left(2 j-\frac{1}{2}\right)} \theta\right) h^{k} d \theta\right. \\
& \left.+\int_{0}^{\frac{\pi}{2}} \sum_{k=0}^{\infty}\left(\sum_{i+j=k} a_{i}^{1} b_{j}^{2} \operatorname{sen}^{\left(2 i-\frac{1}{2}\right)} \theta \cos ^{\left(2 j+\frac{1}{2}\right)} \theta\right) h^{k} d \theta\right] \\
& =\frac{1}{\sqrt{h}}\left[\sum_{k=0}^{\infty}\left(\sum_{i+j=k} a_{i}^{1} b_{j}^{1} \widetilde{I}_{i, j}^{1}\right) h^{k}+h \sum_{k=0}^{\infty}\left(\sum_{i+j=k} a_{i}^{2} b_{j}^{2} \widetilde{I}_{i, j}^{3}\right) h^{k}\right. \\
& \left.+\sqrt{h}\left(\sum_{k=0}^{\infty}\left(\sum_{i+j=k} a_{i}^{2} b_{j}^{1} \widetilde{I}_{i, j}^{4}\right) h^{k}+\sum_{k=0}^{\infty}\left(\sum_{i+j=k} a_{i}^{1} b_{j}^{2} \widetilde{I}_{i, j}^{2}\right) h^{k}\right)\right] \text {, } \\
& \text { onde } \begin{cases}\widetilde{I}_{i, j}^{1}=2 \int_{0}^{\frac{\pi}{2}} \operatorname{sen}^{\left(2 i-\frac{1}{2}\right)} \theta \cos ^{\left(2 j-\frac{1}{2}\right)} \theta d \theta, & \widetilde{I}_{i, j}^{2}=2 \int_{0}^{\frac{\pi}{2}} \operatorname{sen}^{\left(2 i-\frac{1}{2}\right)} \theta \cos ^{\left(2 j+\frac{1}{2}\right)} \theta d \theta, \\
\widetilde{I}_{i, j}^{3}=2 \int_{0}^{\frac{\pi}{2}} \operatorname{sen}^{\left(2 i+\frac{1}{2}\right)} \theta \cos ^{\left(2 j+\frac{1}{2}\right)} \theta d \theta, & \widetilde{I}_{i, j}^{4}=2 \int_{0}^{\frac{\pi}{2}} \operatorname{sen}^{\left(2 i+\frac{1}{2}\right)} \theta \cos ^{\left(2 j-\frac{1}{2}\right)} \theta d \theta .\end{cases}
\end{aligned}
$$

Logo,

$$
T_{1}(h)=t_{0}^{1}+\sum_{k=1}^{\infty} t_{n}^{1} h^{k}=a_{0}^{1} b_{0}^{1} \widetilde{I}_{0,0}^{1}+\sum_{k=1}^{\infty}\left(\sum_{i+j=k} a_{i}^{1} b_{j}^{1} \widetilde{I}_{i, j}^{1}+\sum_{i+j=k-1} a_{i}^{2} b_{j}^{2} \widetilde{I}_{i, j}^{3}\right) h^{k}
$$




$$
T_{2}(h)=\sum_{k=0}^{\infty} t_{n}^{2} h^{k}=\sum_{k=0}^{\infty}\left(\sum_{i+j=k} a_{i}^{2} b_{j}^{1} \widetilde{I}_{i, j}^{4}+\sum_{i+j=k} a_{i}^{1} b_{j}^{2} \widetilde{I}_{i, j}^{2}\right) h^{k}
$$

satisfazem a tese.

Proposição 3.2.1 Seja $T(h)=\frac{1}{\sqrt{h}}\left(T_{1}(h)+\sqrt{h} T_{2}(h)\right)$, onde $T_{1}$ e $T_{2}$ são funções analíticas em zero com $T_{1}(0)>0$. Sejam também $A_{1}$ e $A_{2}$ funções analíticas em zero com $A_{1}(0)>0$. Então a equação integral

$$
T(h)=\int_{0}^{h}\left(\frac{A_{1}(h-z)}{\sqrt[4]{(h-z)^{3}}}+\frac{A_{2}(h-z)}{\sqrt[4]{(h-z)}}\right)\left(\frac{B_{1}(z)}{\sqrt[4]{z^{3}}}+\frac{B_{2}(z)}{\sqrt[4]{z}}\right) d z .
$$

possui uma única solução analítica $\left(B_{1}, B_{2}\right)$ tal que $B_{1}(0)>0$.

Observação: Como nos resultados anteriores, a unicidade no enunciado acima significa que se $B_{1}, B_{2}$ são analíticas definidas em $[0, r)$ satisfazendo (3.11) e $\widetilde{B}_{1}, \widetilde{B}_{2}$ são analíticas em $[0, \widetilde{r})$ satisfazendo (3.11) então $B_{k} \equiv \widetilde{B}_{k}$ em $[0, r) \cap[0, \tilde{r}), k=1,2$.

Demonstração. Vamos inicialmente provar a unicidade de $B_{1}$ e $B_{2}$.

Seja $\left(B_{1}, B_{2}\right)$ solução de (3.11). Seja $\epsilon>0$ tal que $B_{1}(h)=\sum_{n=0}^{\infty} b_{n}^{1} h^{n}, B_{2}(h)=\sum_{n=0}^{\infty} b_{n}^{2} h^{n}$, $A_{1}(h)=\sum_{n=0}^{\infty} a_{n}^{1} h^{n}, A_{2}(h)=\sum_{n=0}^{\infty} a_{n}^{2} h^{n}, T_{1}(h)=\sum_{n=0}^{\infty} t_{n}^{1} h^{n}$ e $T_{2}(h)=\sum_{n=0}^{\infty} t_{n}^{2} h^{n}, h \in[0, \epsilon)$.

Então seguindo os passos da demonstração do Lema 3.2.1 obtemos as igualdades 3.7, e assim

$$
\begin{aligned}
\frac{1}{\sqrt{h}}\left(T_{1}(h)+\sqrt{h} T_{2}(h)\right)=T(h) & =\frac{1}{\sqrt{h}}\left[a_{0}^{1} b_{0}^{1} \widetilde{I}_{0,0}^{1}+\sum_{k=1}^{\infty}\left(\sum_{i+j=k} a_{i}^{1} b_{j}^{1} \widetilde{I}_{i, j}^{1}+\sum_{i+j=k-1} a_{i}^{2} b_{j}^{2} \widetilde{I}_{i, j}^{3}\right) h^{k}\right. \\
& \left.+\sqrt{h}\left(\sum_{k=0}^{\infty}\left(\sum_{i+j=k} a_{i}^{2} b_{j}^{1} \widetilde{I}_{i, j}^{4}+\sum_{i+j=k} a_{i}^{1} b_{j}^{2} \widetilde{I}_{i, j}^{2}\right) h^{k}\right)\right]
\end{aligned}
$$

onde $\widetilde{I}_{i, j}^{1}, \widetilde{I}_{i, j}^{2}, \widetilde{I}_{i, j}^{3}$ e $\widetilde{I}_{i, j}^{4}$ são dados por (3.8), e usando o Lema 2.2 .5 obtemos

$$
\begin{aligned}
& \left\{\begin{array}{l}
t_{0}^{1}=a_{0}^{1} b_{0}^{1} \widetilde{I}_{0,0}^{1} \\
t_{k}^{1}=\left(\sum_{i+j=k} a_{i}^{1} b_{j}^{1} \widetilde{I}_{i, j}^{1}+\sum_{i+j=k-1} a_{i}^{2} b_{j}^{2} \widetilde{I}_{i, j}^{3}\right) \text { se } k \geq 1 .
\end{array}\right. \\
& t_{k}^{2}=\left(\sum_{i+j=k} a_{i}^{2} b_{j}^{1} \widetilde{I}_{i, j}^{4}+\sum_{i+j=k} a_{i}^{1} b_{j}^{2} \widetilde{I}_{i, j}^{2}\right), k \geq 0 .
\end{aligned}
$$


Provemos por indução a unicidade de $\left(b_{k}^{1}\right)_{k \in \mathbb{N}}$ e $\left(b_{k}^{2}\right)_{k \in \mathbb{N}}$.

Para $k=0,(3.12)$ nos dá a unicidade de $b_{0}^{1}$, pois $a_{0}^{1} I_{0,0}^{1} \neq 0$, e em conseqüência (3.13) nos dá a unicidade de $b_{0}^{2}$, pois $a_{0}^{1} I_{0,0}^{2} \neq 0$.

Suponhamos que (3.12) fornece a unicidade de $b_{0}^{1}, b_{1}^{1}, \cdots, b_{k-1}^{1}$ e que em conseqüência (3.13) fornece a unicidade de $b_{0}^{2}, b_{1}^{2}, \cdots, b_{k-1}^{2}$.

Então é imediato que (3.12) fornece a unicidade de $b_{k}^{1}$, pois $a_{0}^{1} I_{0, k}^{1} \neq 0$ e em conseqüência (3.13) fornece a unicidade de $b_{k}^{2}$, pois $a_{0}^{1} I_{0, k}^{2} \neq 0$.

Disto decorre a unicidade local de $B_{1}$ e $B_{2}$, e a unicidade global decorre do princípio do prolongamento analítico.

Vamos mostrar a existência de $B_{1}$ e $B_{2}$, definidas numa vizinhança de zero. Para isso basta mostrarmos que as séries $B_{1}(h)=\sum_{n=0}^{\infty} b_{n}^{1} h^{n}$ e $B_{2}(h)=\sum_{n=0}^{\infty} b_{n}^{2} h^{n}$, onde os coeficientes $b_{n}^{1}$ e $b_{n}^{2}$ são dados por (3.12) e (3.13) convergem em alguma vizinhança de zero, pois então, usando o Lema 1.2.5, mostramos que $\left(B_{1}, B_{2}\right)$ é solução de (3.11) com as mesmas passagens feitas em (3.7).

De (3.12) temos

$t_{n}^{1}=a_{0}^{1} b_{n}^{1} \widetilde{I}_{0, n}^{1}+a_{1}^{1} b_{n-1}^{1} \widetilde{I}_{1, n-1}^{1}+\cdots+a_{n}^{1} b_{0}^{1} \widetilde{I}_{n, 0}^{1}+a_{0}^{2} b_{n-1}^{2} \widetilde{I}_{0, n-1}^{3}+a_{1}^{2} b_{n-2}^{2} \widetilde{I}_{1, n-2}^{3}+\cdots+a_{n-1}^{2} b_{0}^{2} \widetilde{I}_{n-1,0}^{3}$ $\operatorname{logo}$ teremos $b_{n}^{1}$ dado por:

$$
b_{n}^{1}=\frac{t_{n}^{1}-a_{1}^{1} b_{n-1}^{1} \widetilde{I}_{1, n-1}^{1}-\cdots-a_{n}^{1} b_{0}^{1} \widetilde{I}_{n, 0}^{1}-a_{0}^{2} b_{n-1}^{2} \widetilde{I}_{0, n-1}^{3}-\cdots-a_{n-1}^{2} b_{0}^{2} \widetilde{I}_{n-1,0}^{3}}{a_{0}^{1} \widetilde{I}_{0, n}^{1}}
$$

onde, $\quad \widetilde{I}_{i, j}^{1}=2 \int_{0}^{\frac{\pi}{2}} \operatorname{sen}^{\left(2 i-\frac{1}{2}\right)} \theta \cos ^{\left(2 j-\frac{1}{2}\right)} \theta d \theta$ e $\widetilde{I}_{i, j}^{3}=2 \int_{0}^{\frac{\pi}{2}} \operatorname{sen}^{\left(2 i+\frac{1}{2}\right)} \theta \cos ^{\left(2 j+\frac{1}{2}\right)} \theta d \theta$,

Do mesmo modo de (3.13), temos

$$
t_{n}^{2}=a_{0}^{1} b_{n}^{2} \widetilde{I}_{0, n}^{2}+a_{1}^{1} b_{n-1}^{2} \widetilde{I}_{1, n-1}^{2}+\cdots+a_{n}^{1} b_{0}^{2} \widetilde{I}_{n, 0}^{2}+a_{0}^{2} b_{n}^{1} \widetilde{I}_{0, n}^{4}+a_{1}^{2} b_{n-1}^{1} \widetilde{I}_{1, n-1}^{4}+\cdots+a_{n}^{2} b_{0}^{1} \widetilde{I}_{n, 0}^{4}
$$

de onde concluímos que:

$$
\begin{aligned}
& b_{n}^{2}=\frac{t_{n}^{2}-a_{1}^{1} b_{n-1}^{2} \widetilde{I}_{1, n-1}^{2}-\cdots-a_{n}^{1} b_{0}^{2} \widetilde{I}_{n, 0}^{2}-a_{0}^{2} b_{n}^{1} \widetilde{I}_{0, n}^{4}-a_{1}^{2} b_{n-1}^{1} \widetilde{I}_{1, n-1}^{4}-\cdots-a_{n}^{2} b_{0}^{1} \widetilde{I}_{n, 0}^{4}}{a_{0}^{1} \widetilde{I}_{0, n}^{2}},(3 . \\
& \text { onde } \quad \widetilde{I}_{i, j}^{2}=2 \int_{0}^{\frac{\pi}{2}} \operatorname{sen}^{\left(2 i-\frac{1}{2}\right)} \theta \cos ^{\left(2 j+\frac{1}{2}\right)} \theta d \theta e \widetilde{I}_{i, j}^{4}=2 \int_{0}^{\frac{\pi}{2}} \operatorname{sen}^{\left(2 i+\frac{1}{2}\right)} \theta \cos ^{\left(2 j-\frac{1}{2}\right)} \theta d \theta,
\end{aligned}
$$


Do Fato A.0.28 temos $I_{i, j}^{2}=I_{j, i}^{4}$.

Sendo assim de agora em diante usaremos apenas $I_{i, j}^{2}$ no lugar de $I_{j, i}^{4}$ quando estivermos majorando $\left|b_{n}^{2}\right|$.

Suponhamos sem perda de generalidade que $a_{0}^{1}=1$.

Sejam:

$$
\begin{gathered}
\alpha_{2}=\max \left\{\left|b_{0}^{1}\right|,\left|b_{0}^{2}\right|\right\} \\
\alpha_{1}=\max \left\{\left|b_{1}^{1}\right|,\left|b_{1}^{2}\right|\right\} \\
\mathcal{A}=\frac{\left[\Gamma\left(\frac{1}{4}\right)\right]^{2}}{4 \Gamma\left(\frac{1}{2}\right)}, \mathcal{B}=\frac{3}{4} \frac{\Gamma\left(\frac{3}{4}\right) \Gamma\left(\frac{1}{4}\right)}{\Gamma(1)}, \rho=\alpha_{1} \mathcal{A} \text { e } \delta=\alpha_{1} \mathcal{B} .
\end{gathered}
$$

Usando a convergência das séries

$$
\sum_{n=0}^{\infty} a_{n}^{1} h^{n}, \sum_{n=0}^{\infty} a_{n}^{2} h^{n}, \sum_{n=0}^{\infty} t_{n}^{1} h^{n} \text { e } \sum_{n=0}^{\infty} t_{n}^{2} h^{n}
$$

tomemos $\gamma \geq 6$ de modo que

$$
\left|t_{n}^{1}\right| \leq \rho \gamma^{2 n+2}, \quad\left|a_{n}^{1}\right| \leq \gamma^{2 n}, \quad\left|t_{n}^{2}\right| \leq \delta \gamma^{2 n+2} \text { e }\left|a_{n}^{2}\right| \leq \gamma^{2 n+1}
$$

Provaremos por indução em $k$ que

$$
\left|b_{k}^{1}\right| \leq \gamma^{3 k+2}\left(\alpha_{1} k+\alpha_{2}\right) \text { e }\left|b_{k}^{2}\right| \leq \gamma^{3 k+3}\left(\alpha_{1} k+\alpha_{2}\right)
$$

Usando (3.16) e (3.17) e o fato de $\gamma \geq 6$

$$
\begin{aligned}
& \left|b_{0}^{1}\right| \leq \alpha_{2} \gamma^{2}=\left(\alpha_{1} .0+\alpha_{2}\right) \gamma^{2} \\
& \left|b_{0}^{2}\right| \leq \alpha_{2} \gamma^{3} \leq\left(\alpha_{1} .0+\alpha_{2}\right) \gamma^{3} \\
& \left|b_{1}^{1}\right| \leq \alpha_{1} \gamma^{5} \leq \alpha_{1} \gamma^{5}+\alpha_{2} \gamma^{5}=\gamma^{5}\left(\alpha_{1}+\alpha_{2}\right) \\
& \left|b_{1}^{2}\right| \leq \alpha_{1} \gamma^{6} \leq \alpha_{1} \gamma^{6}+\alpha_{2} \gamma^{6}=\gamma^{6}\left(\alpha_{1}+\alpha_{2}\right) .
\end{aligned}
$$

Logo, (3.19) vale para $n=0,1$.

Supondo que as desigualdades (3.19) sejam verdadeiras para $k=1,2, \cdots, n-1, n \geq 2$, vamos provar que elas valem também para $k=n$. 
Assim, usando que para $n \geq 2$ valem $\widetilde{I}_{0, n}^{1}=\widetilde{I}_{n, 0}^{1}, \widetilde{I}_{0, n-1}^{3}=\widetilde{I}_{n-1,0}^{3},\left(\right.$ A.0.29), $\frac{\widetilde{I}_{j, n-j}^{1}}{\widetilde{I}_{1, n-1}^{1}} \leq 1$ se $0<j \leq n-1\left(\right.$ A.0.37 (a)) e $\frac{\widetilde{I}_{j, n-j-1}^{3}}{\widetilde{I}_{1, n-2}^{3}} \leq 1$ se $0<j \leq n-2$ (A.0.37 (e)), e usando também (3.18) e a hipótese de indução em (3.14) obtemos

$$
\begin{aligned}
& \left|b_{n}^{1}\right| \leq \frac{\left|t_{n}^{1}\right|+\left|a_{1}^{1}\right|\left|b_{n-1}^{1}\right| \widetilde{I}_{1, n-1}^{1}+\cdots+\left|a_{n}^{1}\right|\left|b_{0}^{1}\right| \widetilde{I}_{n, 0}^{1}+\left|a_{0}^{2}\right|\left|b_{n-1}^{2}\right| \widetilde{I}_{0, n-1}^{3}+\cdots+\left|a_{n-1}^{2}\right|\left|b_{0}^{2}\right| \widetilde{I}_{n-1,0}^{3}}{\left|a_{0}^{1}\right| \widetilde{I}_{0, n}^{1}} \\
& \leq \frac{\rho \gamma^{2 n+2}}{I_{0, n}^{1}}+\frac{\gamma^{2}\left(\alpha_{1}(n-1)+\alpha_{2}\right) \gamma^{3 n-1}+\cdots+\gamma^{2 n-2}\left(\alpha_{1}+\alpha_{2}\right) \gamma^{5}}{\widetilde{I}_{0, n}^{1}} \widetilde{I}_{1, n-1}^{1}+\gamma^{2 n} \alpha_{2} \gamma^{2} \\
& +\widetilde{I}_{0, n-1}^{3} \frac{\gamma\left(\alpha_{1}(n-1)+\alpha_{2}\right) \gamma^{3 n}+\gamma^{2 n-1} \alpha_{2} \gamma^{3}}{\widetilde{I}_{0, n}^{1}} \\
& +\widetilde{I}_{1, n-2}^{3} \frac{\gamma^{3}\left(\alpha_{1}(n-2)+\alpha_{2}\right) \gamma^{3 n-3}+\cdots+\gamma^{2 n-3}\left(\alpha_{1}+\alpha_{2}\right) \gamma^{6}}{\widetilde{I}_{0, n}^{1}} \\
& \leq \frac{\rho \gamma^{2 n+2}}{\widetilde{I}_{0, n}^{1}}+\frac{\widetilde{I}_{1, n-1}^{1}}{\widetilde{I}_{0, n}^{1}}\left(\alpha_{1} n+2 \alpha_{2}\right) \frac{n-1}{2} \gamma^{3 n+1}+\gamma^{2 n+2} \alpha_{2} \\
& +\frac{\widetilde{I}_{0, n-1}^{3}}{\widetilde{I}_{0, n}^{1}}\left[\gamma^{3 n+1}\left(\alpha_{1} n-\alpha_{1}+\alpha_{2}\right)+\gamma^{2 n+2} \alpha_{2}\right]+\frac{I_{1, n-2}^{3}}{I_{0, n}^{1}} \gamma^{3 n}\left(\alpha_{1} n-\alpha_{1}+2 \alpha_{2}\right) \frac{n-2}{2} \\
& =\gamma^{3 n+2}\left[\frac{\rho}{\gamma^{n} \widetilde{I}_{0, n}^{1}}+\frac{\widetilde{I}_{1, n-1}^{1}}{\widetilde{I}_{0, n}^{1}}\left(\alpha_{1} n+2 \alpha_{2}\right) \frac{n-1}{2 \gamma}+\frac{\alpha_{2}}{\gamma^{n}}\right. \\
& \left.+\frac{\widetilde{I}_{0, n-1}^{3}}{\widetilde{I}_{0, n}^{1}}\left[\frac{\alpha_{1} n-\alpha_{1}+\alpha_{2}}{\gamma}+\frac{\alpha_{2}}{\gamma^{n}}\right]+\frac{\widetilde{I}_{1, n-2}^{3}}{\widetilde{I}_{0, n}^{1}}\left(\alpha_{1} n-\alpha_{1}+\alpha_{2}\right) \frac{n-2}{2 \gamma^{2}}\right] \\
& \leq \gamma^{3 n+2}\left[\frac{\rho}{\gamma^{n} \widetilde{I}_{0, n}^{1}}+\frac{1}{\gamma} \frac{\widetilde{I}_{0, n-1}^{3}}{\widetilde{I}_{0, n}^{1}}\left[\alpha_{1} n+2 \alpha_{2}\right]+\frac{\widetilde{I}_{1, n-1}^{1}}{\widetilde{I}_{0, n}^{1}}\left(\alpha_{1} n+2 \alpha_{2}\right) \frac{n-1}{2 \gamma}+\frac{\alpha_{2}}{\gamma}\right. \\
& \left.+\frac{\widetilde{I}_{1, n-2}^{3}}{\widetilde{I}_{0, n}^{1}}\left(\alpha_{1} n+2 \alpha_{2}\right) \frac{n-2}{2 \gamma}\right] \text {. }
\end{aligned}
$$

Usando que para $n \geq 2$,

$$
\begin{gathered}
\frac{\rho}{\widetilde{I}_{0, n}^{1}} \leq \alpha_{1} n(\text { Fato A.0.37 (a)), } \\
\frac{\widetilde{I}_{0, n-1}^{3}}{\widetilde{I}_{0, n}^{1}} \leq 2 \text { (Fato A.0.37 (b)), } \\
\frac{n-1}{2} \cdot \frac{\widetilde{I}_{1, n-1}^{1}}{\widetilde{I}_{0, n}^{1}} \leq \frac{1}{8} \text { (Fato A.0.37 (c)), }
\end{gathered}
$$




$$
\frac{n-2}{2} \cdot \frac{\widetilde{I}_{1, n-2}^{3}}{\widetilde{I}_{0, n}^{1}} \leq \frac{1}{4}(\text { Fato A.0.37 (d)) }
$$

obtemos

$$
\begin{aligned}
\left|b_{n}^{1}\right| & \leq \gamma^{3 n+2}\left[\frac{1}{\gamma}\left\{\alpha_{1} n+\alpha_{2}+2\left(\alpha_{1} n+2 \alpha_{2}\right)+\frac{1}{8}\left(\alpha_{1} n+2 \alpha_{2}\right)+\frac{1}{4}\left(\alpha_{1} n+2 \alpha_{2}\right)\right\}\right] \\
& \leq \gamma^{3 n+2}\left[\frac{1}{\gamma}\left\{\left(\alpha_{1} n+\alpha_{2}\right)\left(1+4+\frac{1}{4}+\frac{1}{2}\right)\right\}\right] \leq \gamma^{3 n+2}\left(\alpha_{1} n+\alpha_{2}\right), \text { pois } \gamma \geq 6 .
\end{aligned}
$$

Agora usando em (3.15) as desigualdades (3.18), a hipótese de indução $\widetilde{I}_{i, j}^{2}=\widetilde{I}_{j, i}^{4}$ (A.0.28), $\frac{\widetilde{I}_{j, n-j}^{2}}{\widetilde{I}_{1, n-1}^{2}} \leq 1$ se $0<j \leq n-1($ A.0.38 $(c))$.

$$
\begin{aligned}
\left|b_{n}^{2}\right| & \leq \frac{\left|t_{n}^{2}\right|+\left|a_{1}^{1}\right|\left|b_{n-1}^{2}\right| I_{1, n-1}^{2}+\cdots+\left|a_{n}^{1}\right|\left|b_{0}^{2}\right| I_{n, 0}^{2}+\left|a_{0}^{2}\right|\left|b_{n}^{1}\right| I_{0, n}^{4}+\cdots+\left|a_{n}^{2}\right|\left|b_{0}^{1}\right| I_{n, 0}^{4}}{\left|a_{0}^{1}\right| I_{0, n}^{2}} \\
& \leq \frac{\delta \gamma^{2 n+2}}{I_{0, n}^{2}}+\gamma^{2 n} \alpha_{2} \gamma^{3} \frac{I_{n, 0}^{2}}{I_{0, n}^{2}}+\gamma\left(\alpha_{1} n+\alpha_{2}\right) \gamma^{3 n+2} \frac{I_{n, 0}^{2}}{I_{0, n}^{2}}+\gamma^{2 n+1} \alpha_{2} \gamma^{2} \\
& +\left(\gamma^{2}\left(\alpha_{1}(n-1)+\alpha_{2}\right) \gamma^{3 n}+\cdots+\gamma^{2 n-2}\left(\alpha_{1}+\alpha_{2}\right) \gamma^{6}\right) \frac{I_{1, n-1}^{2}}{I_{0, n}^{2}} \\
& +\left(\gamma^{3}\left(\alpha_{1}(n-1)+\alpha_{2}\right) \gamma^{3 n-1}+\cdots+\gamma^{2 n-1}\left(\alpha_{1}+\alpha_{2}\right) \gamma^{5}\right) \frac{I_{1, n-1}^{2}}{I_{0, n}^{2}} \\
& \leq \frac{\delta \gamma^{2 n+2}}{I_{0, n}^{2}}+\gamma^{2 n+3} \alpha_{2} \frac{I_{n, 0}^{2}}{I_{0, n}^{2}}+\gamma^{3 n+3}\left(\alpha_{1} n+\alpha_{2}\right) \frac{I_{n, 0}^{2}}{I_{0, n}^{2}}+\gamma^{2 n+3} \alpha_{2} \\
& +\gamma^{3 n+2}\left(\alpha_{1} n+2 \alpha_{2}\right) \frac{n-1}{2} \frac{I_{1, n-1}^{2}}{I_{0, n}^{2}}+\gamma^{3 n+2}\left(\alpha_{1} n+2 \alpha_{2}\right) \frac{n-1}{2} \frac{I_{1, n-1}^{2}}{I_{0, n}^{2}} \\
& =\gamma^{3 n+3}\left[\frac{\delta}{\gamma^{n+1} I_{0, n}^{2}}+\frac{\alpha_{2}}{\gamma^{n}} \frac{I_{n, 0}^{2}}{I_{0, n}^{2}}+\left(\alpha_{1} n+\alpha_{2}\right) \frac{I_{n, 0}^{2}}{I_{0, n}^{2}}+\frac{\alpha_{2}}{\gamma^{n}}+\frac{1}{\gamma} \frac{I_{1, n-1}^{2}}{I_{0, n}^{2}} \frac{n-1}{2}\left(\alpha_{1} n+2 \alpha_{2}\right)\right. \\
& \left.+\frac{1}{\gamma}\left(\alpha_{1} n+2 \alpha_{2}\right) \frac{n-1}{2} \frac{I_{1, n-1}^{2}}{I_{0, n}^{2}}\right]
\end{aligned}
$$

Então, usando que $n \geq 2$, e o Fato A.0.36, onde
(a) $\frac{n-1}{2} \frac{I_{1, n-1}^{2}}{I_{0, n}^{2}} \leq \frac{1}{8}$
(b) $\frac{\delta}{I_{0, n}^{2}} \leq \alpha_{1} n$
(c) $\frac{I_{n, 0}^{2}}{I_{0, n}^{2}} \leq \frac{1}{3}$ 
obtemos

$$
\begin{aligned}
\left|b_{n}^{2}\right| & \leq \gamma^{3 n+3}\left[\frac{\alpha_{1} n}{\gamma^{n+1}}+\frac{\alpha_{2}}{3 \gamma^{n}}+\frac{\alpha_{1} n+\alpha_{2}}{3}+\frac{\alpha_{2}}{\gamma^{n}}+\frac{1}{8 \gamma}\left(\alpha_{1} n+\alpha_{2}\right)+\frac{1}{8 \gamma}\left(\alpha_{1} n+2 \alpha_{2}\right)\right] \\
& \leq \gamma^{3 n+3}\left[\frac{3 \alpha_{1} n}{3 \gamma}+\frac{\alpha_{2}}{3 \gamma}+\frac{\alpha_{2}}{\gamma}+\frac{\alpha_{1} n+\alpha_{2}}{3}+\frac{1}{2 \gamma}\left(\alpha_{1} n+\alpha_{2}\right)\right] \\
& =\gamma^{3 n+3}\left(\alpha_{1} n+\alpha_{2}\right)\left(\frac{4}{3 \gamma}+\frac{1}{2 \gamma}+\frac{1}{3}\right) \leq \gamma^{3 n+3}\left(\alpha_{1} n+\alpha_{2}\right), \text { pois } \gamma \geq 6 .
\end{aligned}
$$

Observação 3.2.1 Do Lema 2.2.7 com $N=2$ temos que se $l(h)=\sqrt[4]{h}\left(\mathcal{G}_{1}(h)+\sqrt{h} \mathcal{G}_{2}(h)\right)$ com $\mathcal{G}_{1}$ e $\mathcal{G}_{2}$ analíticas em zero tal que $\mathcal{G}_{1}(0)>0$, e $L(y)=y \mathcal{G}_{1}\left(y^{4}\right)+y^{3} \mathcal{G}_{2}\left(y^{4}\right)$, então dadas $G$ analítica em zero da forma $G(y)=b y^{4}+O\left(y^{5}\right), b>0$ tal que $l_{G}=l$, e $\sigma(y)=y-\operatorname{sgn}(y) l(G(y))$, tem-se $G(y)=\left[L^{-1}(y-\sigma(y))\right]^{4}$ numa vizinhança de zero.

Teorema 3.2.1 Seja $T(h)=\frac{1}{\sqrt{h}}\left[T_{1}(h)+\sqrt{h} T_{2}(h)\right]$ onde $T_{1}$ e $T_{2}$ são funções analíticas em zero $T_{1}(0)>0$. Seja também $F(x)=a x^{4}+O\left(x^{5}\right)$ função analítica em zero, com $a>0$. Seja $l_{F}(h)=F_{+}^{-1}(h)-F_{-}^{-1}(h), h \in[0, \bar{h}[$. Então as seguintes afirmações são verdadeiras:

(i) Existe uma única $l(h)=\sqrt[4]{h}\left[\mathcal{L}_{1}(h)+\sqrt{h} \mathcal{L}_{2}(h)\right]$ com $\mathcal{L}_{1}$ e $\mathcal{L}_{2}$ analíticas em zero e $\mathcal{L}_{1}(0)>0$ tal que se $G$ é analítica em zero da forma $G(y)=b y^{4}+O\left(x^{5}\right)$ com $b>0$, e satisfaz $l_{G} \equiv l$, então a origem de (1.2) é um centro com função período $T$.

(ii) Além disso, o conjunto das funções $G$ analíticas em zero da forma $G(y)=b y^{4}+O\left(x^{5}\right)$ com $b>0$ que satisfazem $l_{G}=l$ está em correspondência biunívoca com o conjunto $\Sigma$ das involuções estritas analíticas. Mais concretamente, para cada $\sigma \in \Sigma$ a função correspondente $G$ é dada por $G(y)=\left[L^{-1}(y-\sigma(y))\right]^{4}$ onde $L(y)=y \mathcal{L}_{1}\left(y^{4}\right)+y^{3} \mathcal{L}_{2}\left(y^{4}\right)$ com $\mathcal{L}_{1}$ e $\mathcal{L}_{2}$ dadas em $(i)$.

Demonstração. (i) Pelo Lema 2.1.2

$$
l_{F}^{\prime}(h)=\frac{A_{1}(h)}{\sqrt[4]{h^{3}}}+\frac{A_{2}(h)}{\sqrt[4]{h}} \operatorname{com} A_{1} \text { e } A_{2} \text { analíticas em zero e } A_{1}(0)>0 .
$$

Pela Proposição 3.2 .1 a equação integral

$$
T(h)=\int_{0}^{h}\left(\frac{A_{1}(h-z)}{\sqrt[4]{(h-z)^{3}}}+\frac{A_{2}(h-z)}{\sqrt[4]{h-z}}\right)\left(\frac{B_{1}(z)}{\sqrt[4]{z^{3}}}+\frac{B_{2}(z)}{\sqrt[4]{z}}\right) d z,
$$


possui uma única solução $\left(B_{1}, B_{2}\right)$ analítica em zero com $B_{1}(0)>0$.

Como em (2.21) na demonstração do Teorema 2.2.1, $B_{k}, k=1,2$, pode ser escrita de modo único como

$$
B_{k}(h)=\frac{(2 k-1) \mathcal{L}_{k}(h)}{4}+h \mathcal{L}_{k}^{\prime}(h)
$$

com $\mathcal{L}_{k}$ analítica em zero, $k=1,2$, e é claro nesse caso que $\mathcal{L}_{1}(0)=4 B_{1}(0)>0$.

Definamos $l(h)=\sqrt[4]{h}\left[\mathcal{L}_{1}(h)+\sqrt{h} \mathcal{L}_{2}(h)\right]$.

Seja $G$ analítica em zero da forma $G(y)=b y^{4}+O\left(x^{5}\right) \operatorname{com} b>0$, verificando $l_{G}=l$.

Considere o sistema (1.2) com função de Hamilton $H(x, y)=F(x)+G(y)$. É claro que $H$ tem mínimo na origem e portanto a origem é um centro para (1.2), e como

$$
\begin{aligned}
l_{G}^{\prime}(h) & =l^{\prime}(h)=\frac{1}{4 \sqrt[4]{h^{3}}}\left(\mathcal{L}_{1}(h)+\sqrt{h} \mathcal{L}_{2}(h)\right)+\sqrt[4]{h}\left(\mathcal{L}_{1}^{\prime}(h)+\frac{1}{2 \sqrt{h}} \mathcal{L}_{2}(h)+\sqrt{h} \mathcal{L}_{2}^{\prime}(h)\right) \\
& =\frac{\mathcal{L}_{1}(h)+4 h \mathcal{L}_{1}^{\prime}(h)}{4 \sqrt[4]{h^{3}}}+\frac{3 \mathcal{L}_{2}(h)+4 h \mathcal{L}_{2}^{\prime}(h)}{4 \sqrt[4]{h}} \\
& =\frac{B_{1}(h)}{\sqrt[4]{h^{3}}}+\frac{B_{2}(h)}{\sqrt[4]{h}}
\end{aligned}
$$

decorre que $\int_{0}^{h} l_{F}^{\prime}(h-z) l_{G}^{\prime}(z) d z=\int_{0}^{h}\left(\frac{A_{1}(h-z)}{\sqrt[4]{(h-z)^{3}}}+\frac{A_{2}(h-z)}{\sqrt[4]{h-z}}\right)\left(\frac{B_{1}(z)}{\sqrt[4]{z^{3}}}+\frac{B_{2}(z)}{\sqrt[4]{z}}\right) d z=T(h)$, donde o centro do sistema tem função período $T(h)$.

Isto mostra a existência de $l$ com as propriedades requeridas em $(i)$.

Suponhamos que exista $l_{1}(h)=\sqrt[4]{h}\left[\overline{\mathcal{L}}_{1}(h)+\sqrt{h \mathcal{L}_{2}}(h)\right], \overline{\mathcal{L}}_{1}$ e $\overline{\mathcal{L}}_{2}$ analíticas com $\overline{\mathcal{L}}_{1}(0)>0$, tal que para toda $G$ analítica da forma $G(y)=b y^{4}+O\left(x^{5}\right) \operatorname{com} b>0$ satisfazendo $l_{G}(h)=l_{1}(h)$, a origem de (1.2) seja um centro de período $T$. Então, com cálculos análogos a (3.22) obtemos

$$
l_{G}^{\prime}(h)=l_{1}^{\prime}(h)=\frac{\overline{\mathcal{L}}_{1}(h)+4 h \overline{\mathcal{L}}_{1}^{\prime}(h)}{4 \sqrt[4]{h^{3}}}+\frac{3 \overline{\mathcal{L}}_{2}(h)+4 h \overline{\mathcal{L}}_{2}^{\prime}(h)}{4 \sqrt[4]{h}}=\frac{\bar{B}_{1}(h)}{\sqrt[4]{h^{3}}}+\frac{\bar{B}_{2}(h)}{\sqrt[4]{h}}
$$

onde

$$
\bar{B}_{1}(h)=\frac{1}{4} \overline{\mathcal{L}}_{1}(h)+h \overline{\mathcal{L}}_{1}^{\prime}(h)
$$

e

$$
\bar{B}_{2}(h)=\frac{3}{4} \overline{\mathcal{L}}_{2}(h)+h \overline{\mathcal{L}}_{2}^{\prime}(h) .
$$


Segue que $T(h)=\int_{0}^{h} l_{F}^{\prime}(h-z) l_{G}^{\prime}(z) d z=\int_{0}^{h}\left(\frac{A_{1}(h-z)}{\sqrt[4]{(h-z)^{3}}}+\frac{A_{2}(h-z)}{\sqrt[4]{h-z}}\right)\left(\frac{\bar{B}_{1}(z)}{\sqrt[4]{z^{3}}}+\frac{\bar{B}_{2}(z)}{\sqrt[4]{z}}\right) d z$ e da unicidade de solução de (3.20) segue que $\bar{B}_{1} \equiv B_{1}$ e $\bar{B}_{2} \equiv B_{2}$ numa vizinhança de zero.

Agora, da unicidade de representação de $B_{k}$ na forma dada em (3.21) segue que $\overline{\mathcal{L}}_{1} \equiv \mathcal{L}_{1}$ e $\overline{\mathcal{L}}_{2} \equiv \mathcal{L}_{2}$ numa vizinhança da origem. A unicidade global segue então do princípio do prolongamento analítico [Lim76], [Rud76] e portanto, $l_{1} \equiv l$.

(ii) Seja $l(h)=\sqrt[4]{h}\left[\mathcal{L}_{1}(h)+\sqrt{h} \mathcal{L}_{2}(h)\right]$ dada pelo ítem $(i)$.

Seja $\sigma$ uma involução estrita analítica. A Observação 3.1 .3 garante que existe uma única função $G$ analítica em zero da forma $G(y)=b y^{4}+O\left(y^{5}\right)$ com $b>0$ tal que $l_{G}(h)=G_{+}^{-1}(h)+G_{-}^{-1}(h)=l(h)$ e $\sigma(y)=y-\operatorname{sgn}(y) l(G(y))$. Pela Observação 3.2.1, $G(y)=\left[L^{-1}(y-\sigma(y))\right]^{4}$.

Por outro lado, dada $G(y)=b y^{4}+O\left(y^{5}\right) \operatorname{com} b>0$ função analítica em zero tal que $l_{G}=l$, segue do Observação 3.1 .2 que $\sigma(y)=y-\operatorname{sgn}(y) l(G(y))$ é uma involução estrita analítica. Novamente pela Observação 3.2.1, $G(y)=\left[L^{-1}(y-\sigma(y))\right]^{4}$.

Observação 3.2.2 Note que se o par $\left(\bar{B}_{1}, \bar{B}_{2}\right)$ resolve a equação

$T(h)=\int_{0}^{h}\left(\frac{\bar{B}_{1}(h-z) \bar{B}_{1}(z)}{\sqrt[4]{(h-z)^{3}} \sqrt[4]{z^{3}}}+\frac{\bar{B}_{1}(h-z) \bar{B}_{2}(z)}{\sqrt[4]{(h-z)^{3}} \sqrt[4]{z}}+\frac{\bar{B}_{2}(h-z) \bar{B}_{1}(z)}{\sqrt[4]{(h-z)} \sqrt[4]{z^{3}}}+\frac{\bar{B}_{2}(h-z) \bar{B}_{2}(z)}{\sqrt[4]{(h-z)} \sqrt[4]{z}}\right) d z$

com primeiro membro $T=\bar{T}$ então para todo $\alpha \geq 0$, o par $\left(B_{1}, B_{2}\right)=\left(\sqrt{\alpha} \bar{B}_{1}, \sqrt{\alpha} \bar{B}_{2}\right)$ resolve a mesma equação com primeiro membro $T=\alpha \bar{T}$.

Proposição 3.2.2 Seja $T(h)=\frac{1}{\sqrt{h}}\left(T_{1}(h)+\sqrt{h} T_{2}(h)\right)$, onde $T_{1} T_{2}$ são funções analíticas em zero com $T_{1}(0)>0$. Então a equação integral

$$
T(h)=\int_{0}^{h}\left(\frac{B_{1}(h-z)}{\sqrt[4]{(h-z)^{3}}}+\frac{B_{2}(h-z)}{\sqrt[4]{(h-z)}}\right)\left(\frac{B_{1}(z)}{\sqrt[4]{z^{3}}}+\frac{B_{2}(z)}{\sqrt[4]{z}}\right) d z .
$$

tem uma única solução analítica $\left(B_{1}, B_{2}\right)$ tal que $B_{1}(0)>0$.

Observação: A unicidade no enunciado anterior tem o significado já mencionado antes em várias oportunidades.

Demonstração. Seja $R>0$ tal que $T_{1}(h)=\sum_{n=0}^{\infty} t_{n}^{1} h^{n}$ e $T_{2}(h)=\sum_{n=0}^{\infty} t_{n}^{2} h^{n}$ em $[0, R)$. 
Suponhamos que $B_{1}(h)=\sum_{n=0}^{\infty} b_{n}^{1} h^{n}$ e $B_{2}(h)=\sum_{n=0}^{\infty} b_{n}^{2} h^{n}, h \in[0, \epsilon)$, seja solução de (3.25). Então, como na demonstração da unicidade na Proposição 3.2.1, usando o Fato 1.2.5 e procedendo como em (3.7) obtemos

$$
\begin{aligned}
\frac{1}{\sqrt{h}}\left(T_{1}(h)+\sqrt{h} T_{2}(h)\right) & =\frac{1}{\sqrt{h}}\left[b_{0}^{1} b_{0}^{1} \widetilde{I}_{0,0}^{1}+\sum_{k=1}^{\infty}\left(\sum_{i+j=k} b_{i}^{1} b_{j}^{1} \widetilde{I}_{i, j}^{1}+\sum_{i+j=k-1} b_{i}^{2} b_{j}^{2} \widetilde{I}_{i, j}^{3}\right) h^{k}\right. \\
& \left.+\sqrt{h}\left(\sum_{k=0}^{\infty}\left(\sum_{i+j=k} b_{i}^{2} b_{j}^{1} \widetilde{I}_{i, j}^{4}+\sum_{i+j=k} b_{i}^{1} b_{j}^{2} \widetilde{I}_{i, j}^{2}\right) h^{k}\right)\right],
\end{aligned}
$$

e usando o Lema 2.2.5, obtemos

$$
\begin{aligned}
\left\{\begin{aligned}
t_{0}^{1} & =b_{0}^{1} b_{0}^{1} I_{0,0}^{1} \\
t_{k}^{1} & =\left(\sum_{i+j=k} b_{i}^{1} b_{j}^{1} \widetilde{I}_{i, j}^{1}+\sum_{i+j=k-1} b_{i}^{2} b_{j}^{2} \widetilde{I}_{i, j}^{3}\right) \text { se } k \geq 1 .
\end{aligned}\right. \\
t_{k}^{2}=\left(\sum_{i+j=k} b_{i}^{2} b_{j}^{1} \widetilde{I}_{i, j}^{4}+\sum_{i+j=k} b_{i}^{1} b_{j}^{2} \widetilde{I}_{i, j}^{2}\right) .
\end{aligned}
$$

De $(3.26)$ segue que $\left(b_{0}^{1}\right)^{2}=\frac{t_{0}^{1}}{\widetilde{I}_{0,0}^{1}}$, e como $t_{0}^{1}=T_{1}(0)>0$ obtemos $b_{0}^{1}=\left(\frac{t_{0}^{1}}{\widetilde{I}_{0,0}^{1}}\right)^{\frac{1}{2}}$.

De (3.27) obtemos $b_{0}^{2}=\frac{t_{0}^{2}}{b_{0}^{1}\left(\widetilde{I}_{0,0}^{4}+\widetilde{I}_{0,0}^{2}\right)}=\frac{t_{0}^{2}}{2 b_{0}^{1} \widetilde{I}_{0,0}^{2}}$.

Agora obtemos a unicidade de $\left(b_{n}^{1}\right)_{n \in \mathbb{N}}$ e $\left(b_{n}^{2}\right)_{n \in \mathbb{N}}$ por indução.

Isto prova a unicidade local de $B_{1}$ e $B_{2}$, e pelo princípio do prolongamento analítico segue a unicidade global.

Vamos mostrar a existência de solução $\left(B_{1}, B_{2}\right)$ definida numa vizinhança de zero. Para isso, basta mostrarmos que as séries $B_{1}(h)=\sum_{n=0}^{\infty} b_{n}^{1} h^{n}$ e $B_{2}(h)=\sum_{n=0}^{\infty} b_{n}^{2} h^{n}$, onde os coeficientes são dadas por (3.26) e (3.27), convergem em alguma vizinhança de zero, pois então, como na demonstração da Proposição 3.2 .1 segue que $\left(B_{1}, B_{2}\right)$ é solução de (3.25).

Pela Observação 3.2 .2 podemos supor sem perda de generalidade que $t_{0}^{1}=\widetilde{I}_{0,0}^{1}$ e portanto $b_{0}^{1}=1$.

Segundo o Fato A.0.27 podemos usar que

$$
\widetilde{I}_{0, n}^{1} \geq \frac{\left(\Gamma\left(\frac{1}{4}\right)\right)^{2}}{(4 n-2) \sqrt{\pi}} \text { e } \widetilde{I}_{0, n}^{2} \geq \frac{3 \Gamma\left(\frac{1}{4}\right) \Gamma\left(\frac{3}{4}\right)}{4 n}
$$


então seja $C \geq 1$ tal que $\left|t_{n}^{1}\right| \leq \widetilde{I}_{0, n}^{1} C^{n}$ e $\left|t_{n}^{2}\right| \leq \frac{\widetilde{I}_{0, n}^{2}}{8} C^{n+\frac{1}{2}}$, um tal $C$ existe pois a série $\sum_{n=0}^{\infty} t_{n}^{1} \frac{(4 n-2) \sqrt{\pi}}{\left(\Gamma\left(\frac{1}{4}\right)\right)^{2}} h^{n}$ converge, logo $\left|t_{n}^{1}\right| \leq \frac{\left(\Gamma\left(\frac{1}{4}\right)\right)^{2} C^{n}}{(4 n-2) \sqrt{\pi}} \leq \widetilde{I}_{0, n}^{1} C^{n}$. Do mesmo modo, a série $\sum_{n=0}^{\infty} t_{n}^{2} \frac{4 n}{3 \Gamma\left(\frac{1}{4}\right) \Gamma\left(\frac{3}{4}\right)} h^{n}$ converge e portanto $\left|t_{n}^{2}\right| \leq \frac{3 \Gamma\left(\frac{1}{4}\right) \Gamma\left(\frac{3}{4}\right)}{4 n} C^{n+\frac{1}{2}} \leq \frac{\widetilde{I}_{0, n}^{2}}{8} C^{n+\frac{1}{2}}$

Afirmamos que

$$
\left|b_{k}^{1}\right| \leq C^{k} \text { e }\left|b_{k}^{2}\right| \leq \frac{C^{k+\frac{1}{2}}}{4}
$$

provemos esta afirmação por indução em $k$.

$$
\begin{aligned}
& \text { Para } k=0,\left|b_{0}^{1}\right|=1=C^{0} \text { e }\left|b_{0}^{2}\right| \leq \frac{\widetilde{I}_{0,0}^{2}}{16 \widetilde{I}_{0,0}^{2}} C^{\frac{1}{2}}<\frac{C^{\frac{1}{2}}}{4} . \\
& \text { Para } k=1 \text {, usaremos } \frac{\widetilde{I}_{0,0}^{3}}{\widetilde{I}_{0,1}^{1}} \leq 2 \text { (Fato A.0.34) e } \frac{\widetilde{I}_{1,0}^{2}}{\widetilde{I}_{0,1}^{2}} \leq \frac{1}{3} \text { (Fato A.0.38 }(a) \text { ). } \\
& \left|b_{1}^{1}\right| \leq \frac{\left|t_{1}^{1}\right|}{2 \widetilde{I}_{0,1}^{1}}+2 \frac{\left|b_{0}^{2}\right|^{2} I_{0,0}^{3}}{2 \widetilde{I}_{0,1}^{1}} \leq \frac{\widetilde{I}_{0,1}^{1} C}{2 \widetilde{I}_{0,1}^{1}}+\frac{C}{8} \leq C \quad \mathrm{e} \\
& \left|b_{1}^{2}\right| \leq \frac{\left|t_{1}^{2}\right|}{2 \widetilde{I}_{0,1}^{2}}+\frac{2\left|b_{0}^{2}\right|\left|b_{1}^{1}\right| \widetilde{I}_{1,0}^{2}}{2 \widetilde{I}_{0,1}^{2}} \leq \frac{\widetilde{I}_{0,1}^{2} C^{1+\frac{1}{2}}}{16 \widetilde{I}_{0,1}^{2}}+\frac{C^{1+\frac{1}{2}}}{12} \leq \frac{C^{1+\frac{1}{2}}}{4}
\end{aligned}
$$

Logo a afirmação é verdadeira para $k=0,1$.

Suponhamos que a afirmação seja verdadeira para $k=0,1,2, \cdots, n-1$ e provemos para $k=n \geq 2$.

Pelo Fato A.0.37 temos
(a) para $0<j \leq n-1$, temos $\frac{\widetilde{I}_{n-j, j}^{1}}{\widetilde{I}_{1, n-1}^{1}} \leq 1$
(b) $\frac{\widetilde{I}_{0, n-1}^{3}}{\widetilde{I}_{0, n}^{1}} \leq 2$, para $n \geq 2 ;$
(c) $\frac{\widetilde{I}_{1, n-1}^{1}}{\widetilde{I}_{0, n}^{1}} \leq \frac{1}{4\left(n-\frac{3}{4}\right)}$, para $n \geq 1$;
(d) $\frac{\widetilde{I}_{1, n-2}^{3}}{\widetilde{I}_{0, n}^{1}}<\frac{3}{4\left(n-\frac{3}{4}\right)}$, para $n \geq 3$;
(e) para $0<i \leq n-2$, temos $\frac{\widetilde{I}_{i, n-1-i}^{3}}{\widetilde{I}_{1, n-2}^{3}} \leq 1$, 
e usando também que $I_{i, j}^{1}=I_{j, i}^{1}$ e $I_{i, j}^{3}=I_{j, i}^{3}$ em (3.26), obtemos: No caso $n=2$;

$$
\begin{aligned}
\left|b_{2}^{1}\right| \leq & \frac{\left|t_{2}^{1}\right|}{2 I_{0,2}^{1}}+\frac{2\left|b_{1}^{1}\right|\left|b_{1}^{1}\right| \widetilde{I}_{1,1}^{1}}{2 \widetilde{I}_{0,2}^{1}}+\frac{2\left|b_{0}^{2}\right|\left|b_{1}^{2}\right| \widetilde{I}_{0,1}^{3}}{2 \widetilde{I}_{0,2}^{1}} \\
\leq & \frac{\widetilde{I}_{0,2}^{1} C^{2}}{2 \widetilde{I}_{0,2}^{1}}+\frac{C^{2} \widetilde{I}_{1,1}^{1}}{\widetilde{I}_{0,2}^{1}}+\frac{\frac{C^{\frac{1}{2}}}{4} \frac{C^{1+\frac{1}{2}}}{4} \widetilde{I}_{0,1}^{3}}{\widetilde{I}_{0,2}^{1}} \leq C^{2}\left[\frac{1}{2}+\frac{1}{5}+\frac{1}{16} 2\right]<C^{2} \\
\left|b_{n}^{1}\right| \leq & \frac{\left|t_{n}^{1}\right|}{2 I_{0, n}^{1}}+\frac{\left|b_{1}^{1}\right|\left|b_{n-1}^{1}\right| \widetilde{I}_{1, n-1}^{1}+\left|b_{2}^{1}\right|\left|b_{n-2}^{1}\right| \widetilde{I}_{2, n-2}^{1}+\cdots+\left|b_{n-2}^{1}\right|\left|b_{2}^{1}\right| \widetilde{I}_{n-2,2}^{1}+\left|b_{n-1}^{1}\right|\left|b_{1}^{1}\right| \widetilde{I}_{n-1,1}^{1}}{2 \widetilde{I}_{0, n}^{1}} \\
+ & \frac{\left|b_{0}^{2}\right|\left|b_{n-1}^{2}\right| \widetilde{I}_{0, n-1}^{3}+\left|b_{1}^{2}\right|\left|b_{n-2}^{2}\right| \widetilde{I}_{1, n-2}^{3}+\cdots+\left|b_{n-2}^{2}\right|\left|b_{1}^{2}\right| \widetilde{I}_{n-2,1}^{3}+\left|b_{n-1}^{2}\right|\left|b_{0}^{2}\right| I_{n-1,0}^{3}}{2 I_{0, n}^{1}} \\
\leq & \frac{I_{0, n}^{1} C^{n}}{2 I_{0, n}^{1}}+\left(\left|b_{1}^{1}\right|\left|b_{n-1}^{1}\right|+\left|b_{2}^{1}\right|\left|b_{n-2}^{1}\right|+\cdots+\left|b_{n-2}^{1}\right|\left|b_{2}^{1}\right|+\left|b_{n-1}^{1}\right|\left|b_{1}^{1}\right|\right) \frac{\widetilde{I}_{1, n-1}^{1}}{2 \widetilde{I}_{0, n}^{1}} \\
+ & \left(\left|b_{1}^{2}\right|\left|b_{n-2}^{2}\right|+\cdots+\left|b_{n-2}^{2}\right|\left|b_{1}^{2}\right|\right) \frac{\widetilde{I}_{1, n-2}^{3}}{2 I_{0, n}^{1}}+\left|b_{0}^{2}\right|\left|b_{n-1}^{2}\right| \frac{\widetilde{I}_{0, n-1}^{3}}{\widetilde{I}_{0, n}^{1}} \\
\leq & \frac{C^{n}}{2}+\left(C^{1} C^{n-1}+C^{2} C^{n-2}+\cdots+C^{n-2} C^{2}+C^{n-1} C^{1}\right) \frac{\widetilde{I}_{1, n-1}^{1}}{2 \widetilde{I}_{0, n}^{1}} \\
+ & \left(\frac{C^{\frac{3}{2}} C^{n-\frac{3}{2}}}{16}+\frac{C^{\frac{5}{2}} C^{n-\frac{5}{2}}}{16}+\cdots+\frac{C^{n-\frac{5}{2}}}{16} C^{\frac{5}{2}}+\frac{C^{n-\frac{3}{2}} C^{\frac{3}{2}}}{16}\right) \frac{\widetilde{I}_{1, n-2}^{3}}{2 \widetilde{I}_{0, n}^{1}}+\frac{C^{\frac{1}{2}} C^{n-\frac{1}{2}}}{16} \frac{I_{0, n-1}^{3}}{I_{0, n}^{1}} \\
\leq & C^{n}\left[\frac{1}{2}+(n-1) \frac{1}{8\left(n-\frac{3}{4}\right)}+\frac{(n-2)}{16} \frac{3}{8\left(n-\frac{3}{4}\right)}+\frac{2}{16}\right] \leq C^{n}\left[\frac{1}{2}+\frac{1}{8}+\frac{3}{16}+\frac{1}{8}\right] \leq C^{n} .
\end{aligned}
$$

Para majorar $\left|b_{n}^{2}\right|$, usaremos os seguintes resultados dados pelo Fato A.0.38:

(a) $\frac{\widetilde{I}_{n, 0}^{2}}{\widetilde{I}_{0, n}^{2}} \leq \frac{1}{3}, n \geq 1$, (b) $\frac{\widetilde{I}_{1, n-1}^{2}}{\widetilde{I}_{0, n}^{2}}=\frac{1}{4\left(n-\frac{1}{4}\right)}$, para $n \geq 1$, (c) $\frac{\widetilde{I}_{n-j, j}^{2}}{\widetilde{I}_{1, n-1}^{2}} \leq 1$ para $0<j \leq n-1$.

Substituindo esses resultados em (3.27), obtemos:

$$
\begin{aligned}
\left|b_{n}^{2}\right| & \leq \frac{\left|t_{n}^{2}\right|}{2 I_{0, n}^{2}}+\frac{\left|b_{1}^{1}\right|\left|b_{n-1}^{2}\right| \widetilde{I}_{1, n-1}^{2}+\cdots+\left|b_{n}^{1}\right|\left|b_{0}^{2}\right| \widetilde{I}_{n, 0}^{2}+\left|b_{0}^{2}\right|\left|b_{n}^{1}\right| \widetilde{I}_{n, 0}^{2}+\cdots+\left|b_{n-1}^{2}\right|\left|b_{1}^{1}\right| \widetilde{I}_{1, n-1}^{2}}{2 I_{0, n}^{2}} \\
& =\frac{\left|t_{n}^{2}\right|}{2 I_{0, n}^{2}}+2\left(\left|b_{1}^{1}\right|\left|b_{n-1}^{2}\right|+\left|b_{2}^{1}\right|\left|b_{n-2}^{2}\right|+\cdots+\left|b_{n-1}^{1}\right|\left|b_{1}^{2}\right|\right) \frac{I_{1, n-1}^{2}}{2 I_{0, n}^{2}}+\left|b_{n}^{1}\right|\left|b_{0}^{2}\right| \frac{\widetilde{I}_{n, 0}^{2}}{\widetilde{I}_{0, n}^{2}} \\
& \left.\leq \frac{\widetilde{I}_{0, n}^{2} C^{n+\frac{1}{2}}}{16 \widetilde{I}_{0, n}^{2}}+\left(C \frac{C^{n-\frac{1}{2}}}{4}+C^{2} \frac{C^{n-\frac{3}{2}}}{4}+\cdots+C^{n-1} \frac{C^{\frac{3}{2}}}{4}\right) \frac{1}{4\left(n-\frac{1}{4}\right)}+C^{n} \frac{C^{\frac{1}{2}}}{4}\right) \frac{1}{3} \\
& =\frac{C^{n+\frac{1}{2}}}{4}\left[\frac{1}{4}+(n-1) \frac{1}{4\left(n-\frac{1}{4}\right)}+\frac{1}{3}\right] \leq \frac{C^{n+\frac{1}{2}}}{4} \frac{10}{12}<\frac{C^{n+\frac{1}{2}}}{4} .
\end{aligned}
$$


Portanto, as séries convergem no intervalo $\left(-\frac{1}{C}, \frac{1}{C}\right)$, além disso se $T_{2}(0)=t_{0}^{2}>0$, teremos por $(3.27)$ que $B(0)=b_{0}^{2}>0$.

Teorema 3.2.2 Considere o sistema (1.2) com $f \equiv g$. Seja $T(h)=\frac{1}{\sqrt{h}}\left[T_{1}(h)+\sqrt{h} T_{2}(h)\right]$, onde $T_{1}$ e $T_{2}$ são funções analíticas em zero satisfazendo $T_{1}(0)>0$. Então as seguintes afirmações são verdadeiras:

(i) Existe uma única $l(h)=\sqrt[4]{h}\left[\mathcal{L}_{1}(h)+\sqrt{h} \mathcal{L}_{2}(h)\right]$ com $\mathcal{L}_{1}$ e $\mathcal{L}_{2}$ analíticas em zero e $\mathcal{L}_{1}(0)>0$ tal que se $F$ e $G$ são analiticas em zero, com a forma $F(u)=G(u)=a u^{4}+O\left(u^{5}\right)$ com $a>0$ e satisfazem $l_{F}=l_{G}=l$, então a origem de (1.2) é um centro com função período $T$. Além disso se $T_{2}(0)>0$ então $\mathcal{L}_{2}(0)>0$.

(ii) Além disso, o conjunto das funções $F$ analíticas em zero da forma $F(h)=a h^{4}+O\left(h^{5}\right)$ com $a>0$ que satisfazem $l_{F}=l$ está em correspondência biunívoca com o conjunto $\Sigma$ das involuções estritas analíticas. Mais concretamente, para cada $\sigma \in \Sigma$ a função correspondente $F$ é dada por $F(x)=\left[L^{-1}(x-\sigma(x))\right]^{4}$ onde $L(x)=x \mathcal{L}_{1}\left(x^{4}\right)+x^{3} \mathcal{L}_{2}\left(x^{4}\right)$ com $\mathcal{L}_{1}$ e $\mathcal{L}_{2}$ dadas em (i).

Demonstração. (i) Pela Proposição 3.2 .2 a equação integral

$$
T(h)=\int_{0}^{h}\left(\frac{B_{1}(h-z)}{\sqrt[4]{(h-z)^{3}}}+\frac{B_{2}(h-z)}{\sqrt[4]{h-z}}\right)\left(\frac{B_{1}(z)}{\sqrt[4]{z^{3}}}+\frac{B_{2}(z)}{\sqrt[4]{z}}\right) d z
$$

possui uma única solução $\left(B_{1}, B_{2}\right)$ analítica em zero com $B_{1}(0)>0$.

Como em (2.21) na demonstração do Teorema 2.2.1 (e como na demonstração do Teorema 3.2.1), conclui-se que $B_{k}, k=1,2$, pode ser escrita de modo único como

$$
B_{k}(h)=\frac{(2 k-1) \mathcal{L}_{k}(h)}{4}+h \mathcal{L}_{k}^{\prime}(h),
$$

com $\mathcal{L}_{k}$ analítica em zero e $k=1,2$ e $\mathcal{L}_{1}(0)=4 B_{1}(0)>0$.

Também como na demonstração do Teorema 3.2.1 definimos $l(h)=\sqrt[4]{h}\left[\mathcal{L}_{1}(h)+\sqrt{h} \mathcal{L}_{2}(h)\right]$.

Sejam $F \equiv G$ analíticas em zero da forma $F(u)=G(u)=a u^{4}+O\left(u^{5}\right)$ com $a>0$, verificando $l_{F}=l_{G}=l$.

Considere o sistema (1.2) com função de Hamilton $H(x, y)=F(x)+G(y)$. É claro que $H$ tem mínimo na origem e portanto a origem é um centro para (1.2).

Também como na demonstração do Teorema 3.2.1 concluímos que

$$
l_{F}^{\prime}(h)=l_{G}^{\prime}(h)=l^{\prime}(h)=\frac{B_{1}(h)}{\sqrt[4]{h^{3}}}+\frac{B_{2}(h)}{\sqrt[4]{h}},
$$


portanto,

$$
\int_{0}^{h} l_{F}^{\prime}(h-z) l_{G}^{\prime}(z) d z=\int_{0}^{h}\left(\frac{B_{1}(h-z)}{\sqrt[4]{(h-z)^{3}}}+\frac{B_{2}(h-z)}{\sqrt[4]{h-z}}\right)\left(\frac{B_{1}(z)}{\sqrt[4]{z^{3}}}+\frac{B_{2}(z)}{\sqrt[4]{z}}\right) d z=T(h),
$$

donde o centro do sistema tem função período $T(h)$.

Isto mostra a existência de $l$ com as propriedades requeridas em $(i)$.

Suponhamos que exista $l_{1}(h)=\sqrt[4]{h}\left[\overline{\mathcal{L}}_{1}(h)+\sqrt{h \mathcal{L}_{2}}(h)\right], \overline{\mathcal{L}}_{1}$ e $\overline{\mathcal{L}}_{2}$ analíticas com $\overline{\mathcal{L}}_{1}(0)>0$, tal que para todo par $(F, G)$ de funções analíticas em zero e da forma $F(u)=G(u)=a u^{4}+O\left(u^{5}\right)$ com $a>0$ satisfazendo $l_{F}(h)=l_{G}(h)=l_{1}(h)$, a origem de (1.2) seja um centro de período $T$. Então com cálculos análogos a (3.22) obtemos

$$
l_{F}^{\prime}(h)=l_{G}^{\prime}(h)=\frac{\overline{\mathcal{L}}_{1}(h)+4 h \overline{\mathcal{L}}_{1}^{\prime}(h)}{4 \sqrt[4]{h^{3}}}+\frac{3 \overline{\mathcal{L}}_{2}(h)+4 h \overline{\mathcal{L}}_{2}^{\prime}(h)}{4 \sqrt[4]{h}} .
$$

e tomando $\bar{B}_{1}$ e $\bar{B}_{2}$ como em (3.23) e (3.24) respectivamente segue que

$$
T(h)=\int_{0}^{h} l_{F}^{\prime}(h-z) l_{G}^{\prime}(z) d z=\int_{0}^{h}\left(\frac{\bar{B}_{1}(h-z)}{\sqrt[4]{(h-z)^{3}}}+\frac{\bar{B}_{2}(h-z)}{\sqrt[4]{h-z}}\right)\left(\frac{\bar{B}_{1}(z)}{\sqrt[4]{z^{3}}}+\frac{\bar{B}_{2}(z)}{\sqrt[4]{z}}\right) d z
$$

e de modo análogo à demonstração do Teorema 3.2.1, da unicidade de solução de (3.28) e da unicidade de representação de $B_{k}$ na forma dada em (3.29) decorrerá que $l_{1}=l$.

(ii) A demonstração desta afirmação é exatamente igual à feita em (ii) no Teorema 3.2.1, bastando trocar $G$ por $F$.

Corolário 3.2.1 O sistema (1.2) não tem centro isócrono quando $F$ e $G$ são analíticas da forma $F(u)=G(u)=a u^{4}+O\left(u^{5}\right)$ com $a>0$.

Demonstração. Decorre imediatamente do Lema 3.2.1.

Corolário 3.2.2 Seja T a função período de (1.2) quando $F$ e $G$ são analíticas em zero da forma $F(u)=G(u)=a u^{4}+O\left(u^{5}\right)$, com $a>0$. Suponhamos que $\sqrt{h} T(h)$ seja constante. Então $l_{F}(h)=l_{G}(h)=a \sqrt[4]{h} \operatorname{com} a>0$.

Demonstração. Pelo Lema 3.2.1

$$
\sqrt{h} T(h)=T_{1}(h)+\sqrt{h} T_{2}(h)
$$

com $T_{1}$ e $T_{2}$ analíticas em zero e $T_{1}(0)>0$. 
Sendo assim, se $\sqrt{h} T(h)$ é constante então $T_{1}(h) \equiv T_{1}(0)>0$ e $T_{2}(h) \equiv 0$.

Pela Observação 3.1.1,

$$
l_{F}(h)=l_{G}(h)=\sqrt[4]{h}\left(\mathcal{G}_{1}(h)+\sqrt{h} \mathcal{G}_{2}(h)\right), \operatorname{com} \mathcal{G}_{1}(0)>0,
$$

e $\mathcal{G}_{1}, \mathcal{G}_{2}$ analíticas em zero. Além disso, $l_{F}^{\prime}(h)=l_{G}^{\prime}(h)=\frac{B_{1}(h)}{\sqrt[4]{h^{3}}}+\frac{B_{2}(h)}{\sqrt[4]{h}}$, onde $B_{1}$ e $B_{2}$ são analíticas em zero com $B_{1}(0)>0$ tal que

$$
B_{k}(h)=\frac{(2 k-1) \mathcal{G}_{k}(h)}{4}+h \mathcal{G}_{k}^{\prime}(h), k=1,2 .
$$

Como $B_{k}$ e $\mathcal{G}_{k}$ são analíticas em zero, sejam $B_{k}(h)=\sum_{n=0}^{\infty} b_{n}^{k} h^{n}$ e $\mathcal{G}_{k}(h)=\sum_{n=0}^{\infty} c_{n}^{k} h^{n}$.

Por (3.9) e (3.10) na demonstração do Lema 3.2.1 temos

$$
\begin{aligned}
0<T_{1}(0) & =T_{1}(h)=\left(b_{0}^{1}\right)^{2} I_{0,0}^{1}+\sum_{k=1}^{\infty}\left(\sum_{i+j=k} b_{i}^{1} b_{j}^{1} \widetilde{I}_{i, j}^{1}+\sum_{i+j=k-1} b_{i}^{2} b_{j}^{2} \widetilde{I}_{i, j}^{3}\right) h^{k} \\
0 & =T_{2}(h)=\sum_{k=0}^{\infty}\left(\sum_{i+j=k} b_{i}^{2} b_{j}^{1} \widetilde{I}_{i, j}^{4}+\sum_{i+j=k} b_{i}^{1} b_{j}^{2} \widetilde{I}_{i, j}^{2}\right) h^{k}
\end{aligned}
$$

Concluímos com isso que $b_{0}^{1}=\left(\frac{C}{I_{0,0}^{1}}\right)^{\frac{1}{2}}, b_{n}^{1}=0, \forall n \geq 1$ e $b_{n}^{2}=0, \forall n \geq 1$.

Logo, $B_{1}(h)=b_{0}^{1}=\left(\frac{C}{I_{0,0}^{1}}\right)^{\frac{1}{2}}$ e $B_{2}(h)=0$; disto e de (3.31) decorre imediatamente que $\mathcal{G}_{1}(h)=a>0$ e $\mathcal{G}_{2}(h)=0$, e portanto voltando a (3.30) obtemos $l_{F}(h)=l_{G}(h)=a \sqrt[4]{h}, a>0$. 



\section{Apêndice}

Neste apêndice estão alguns resultados que foram usados na demonstração de diversos resultados deste trabalho. Algumas fórmulas e relações das funções $\beta$ e $\Gamma$ podem ser encontradas em [Kre88], [Spi74] .

\section{Definição A.0.1}

$$
I_{i, j}=2 \int_{0}^{\frac{\pi}{2}} \operatorname{sen}^{2 i} t \cos ^{2 j} t d t=\beta\left(i+\frac{1}{2}, j+\frac{1}{2}\right)=\frac{\Gamma\left(i+\frac{1}{2}\right) \Gamma\left(j+\frac{1}{2}\right)}{\Gamma(i+j+1)}, i, j \in \mathbb{N} .
$$

A função $\Gamma$ tem as seguintes propriedades: $\Gamma(s+1)=s \Gamma(s), s \in \mathbb{R}, \Gamma(1)=1$, $\Gamma\left(\frac{1}{2}\right)=\sqrt{\pi}$.

Abaixo estão algumas propriedades de $I_{i, j}$.

Fato A.0.1 $I_{i, j}=I_{j, i}, \forall i, j \in \mathbb{N}$.

Fato A.0.2 $I_{0,0}=\pi$ e $I_{0, n} \geq \frac{\pi}{2 n}, \forall n \geq 1$.

Demonstração. $I_{0,0}=\pi$ é imediato da Definição A.0.1

$$
\begin{aligned}
I_{0, n} & =\frac{\Gamma\left(\frac{1}{2}\right) \Gamma\left(n+\frac{1}{2}\right)}{\Gamma(n+1)}=\frac{\sqrt{\pi}\left(n-\frac{1}{2}\right)\left(n-\frac{3}{2}\right)\left(n-\frac{5}{2}\right) \cdots \frac{3}{2} \frac{1}{2} \Gamma\left(\frac{1}{2}\right)}{n(n-1)(n-2) \cdots 2.1 \Gamma(1)} \\
& =\frac{\pi}{2 n} \frac{(2 n-1)(2 n-3) \cdots 3.1}{(2 n-2)(2 n-4) \cdots 3.2} \geq \frac{\pi}{2 n} .
\end{aligned}
$$

Fato A.0.3 Se $n \geq 3,2 j \leq n$ e $2 \leq j \leq n-1$ então $I_{j, n-j} \leq I_{j-1, n-j+1}$. 
Demonstração. De fato,

$$
\begin{aligned}
& \frac{I_{j, n-j}}{I_{j-1, n-j+1}}=\frac{\Gamma\left(\frac{1}{2}+j\right) \Gamma\left(\frac{1}{2}+n-j\right)}{\Gamma(n+1)} \frac{\Gamma(n+1)}{\Gamma\left(\frac{1}{2}+j-1\right) \Gamma\left(\frac{1}{2}+n-j+1\right)} \\
= & \frac{\Gamma\left(j-\frac{1}{2}+1\right) \Gamma\left(\frac{1}{2}+n-j\right)}{\Gamma\left(j-\frac{1}{2}\right) \Gamma\left(\frac{1}{2}+n-j+1\right)}=\frac{\left(j-\frac{1}{2}\right) \Gamma\left(j-\frac{1}{2}\right) \Gamma\left(\frac{1}{2}+n-j\right)}{\Gamma\left(j-\frac{1}{2}\right)\left(\frac{1}{2}+n-j\right) \Gamma\left(\frac{1}{2}+n-j\right)}=\frac{2 j-1}{1+2 n-2 j} .
\end{aligned}
$$

Como $2 j \leq n \Rightarrow 2 j \leq n+1 \Rightarrow 4 j \leq 2 n+2 \Rightarrow 4 j-1 \leq 2 n+1 \Rightarrow 2 j-1 \leq 1+2 n-2 j$, temos que

$$
I_{j, n-j} \leq I_{j-1, n-j+1} .
$$

Fato A.0.4 Se $n \geq 2,1 \leq j \leq n-1$ e $2 j \leq n$ então $I_{j, n-j} \leq I_{1, n-1}$.

Demonstração. $j=1$ : segue imediatamente do Fato (A.0.1)

$n=2$ : é imediato, pois necessariamente $j=1$.

$n \geq 3, j \geq 2$ : basta usar o Fato (A.0.3) repetidamente.

Fato A.0.5 Se $n \geq 2$ e $1 \leq j \leq n-1$ então $I_{j, n-j} \leq I_{1, n-1}$.

Demonstração. $2 j \leq n$ : segue do Fato (A.0.4)

$2 j>n$ : então $2(n-j)<n$ e $1 \leq n-j \leq n-1$, e usando os Fatos (A.0.1) e (A.0.4) vem que

$$
I_{j, n-j}=I_{n-j, j} \leq I_{1, n-1}
$$

Fato A.0.6 se $n \geq 1$ então $I_{1, n-1}=\frac{1}{2 n-1} I_{0, n}$.

Demonstração. De fato,

$$
\frac{I_{1, n-1}}{I_{0, n}}=\frac{\Gamma\left(\frac{1}{2}+1\right) \Gamma\left(\frac{1}{2}+n-1\right)}{\Gamma(n+1)} \frac{\Gamma(n+1)}{\Gamma\left(\frac{1}{2}\right) \Gamma\left(\frac{1}{2}+n\right)}=\frac{\frac{1}{2} \Gamma\left(\frac{1}{2}\right) \Gamma\left(n-\frac{1}{2}\right)}{\Gamma\left(\frac{1}{2}\right) \Gamma\left(n-\frac{1}{2}\right)\left(n-\frac{1}{2}\right)}=\frac{1}{2 n-1} .
$$

Fato A.0.7 se $n \geq 1$ então $I_{0, n} \leq \frac{\pi}{2} \leq \pi$. 
Demonstração.

$$
\begin{aligned}
I_{0, n} & =\frac{\Gamma\left(\frac{1}{2}\right) \Gamma\left(n+\frac{1}{2}\right)}{\Gamma(n+1)}=\frac{\sqrt{\pi}\left(n-\frac{1}{2}\right)\left(n-\frac{3}{2}\right)\left(n-\frac{5}{2}\right) \cdots \frac{3}{2} \frac{1}{2} \Gamma\left(\frac{1}{2}\right)}{n(n-1)(n-2) \cdots 2.1 \Gamma(1)} \\
& =\pi \frac{(2 n-1)(2 n-3)(2 n-5) \cdots 3.1}{2 n(2 n-2)(2 n-4) \cdots 3.2} \leq \frac{\pi}{2} \leq \pi .
\end{aligned}
$$

Observação: Nos Fatos de A.0.8 a A.0.18, abaixo usaremos a seguinte

Definição A.0.2 Para $N \in \mathbb{N}^{*}$ definimos

$$
I_{i, j}^{q}=2 \int_{0}^{\frac{\pi}{2}} \operatorname{sen}^{2 i} t \cos ^{2 j-\frac{N-2 q+1}{N}} t d t=\beta\left(i+\frac{1}{2}, j+\frac{2 q-1}{2 N}\right)=\frac{\Gamma\left(i+\frac{1}{2}\right) \Gamma\left(j+\frac{2 q-1}{2 N}\right)}{\Gamma\left(i+j+\frac{N+2 q-1}{2 N}\right)},
$$

$q=1,2, \cdots, N$ e $i, j \in \mathbb{N}$

Fato A.0.8 Seja $1 \leq q \leq N$. Se $1 \leq j \leq n-1$ então $I_{j, n-j}^{q} \leq I_{0, n}^{q}$.

Demonstração. De fato, como $0<\frac{2 q-1}{2 N}<1$ e $n>j \geq 1$, temos $n \geq 2$ e chamando $p=2 q-1$ temos

$$
\begin{aligned}
\frac{I_{j, n-j}^{q}}{I_{0, n}^{q}} & =\frac{\Gamma\left(j+\frac{1}{2}\right) \Gamma\left(n-j+\frac{p}{2 N}\right)}{\Gamma\left(\frac{1}{2}\right) \Gamma\left(n+\frac{p}{2 N}\right)} \\
& =\frac{\left(j-\frac{1}{2}\right)\left(j-\frac{3}{2}\right)\left(j-\frac{5}{2}\right) \cdots \frac{3}{2} \frac{1}{2} \Gamma\left(\frac{1}{2}\right) \Gamma\left(n-j+\frac{p}{2 N}\right)}{\Gamma\left(\frac{1}{2}\right)\left(n-1+\frac{p}{2 N}\right)\left(n-2+\frac{p}{2 N}\right) \cdots\left(n-j+1+\frac{p}{2 N}\right)\left(n-j+\frac{p}{2 N}\right) \Gamma\left(n-j+\frac{p}{2 N}\right)} \\
& \leq \frac{(2 j-1)(2 j-3)(2 j-5) \cdots 3.1}{(2 n-2)(2 n-4)(2 n-6) \cdots(2 n-2(j-1))(2 n-2 j)} \leq 1,
\end{aligned}
$$

pois $n>j$.

Fato A.0.9 Se $n \in \mathbb{N}^{*}$ e $q=1,2, \cdots, N$ então

$$
I_{0, n}^{q} \geq I_{0, n+1} \geq \frac{\pi}{2 n+2}, \forall n \geq 0 .
$$

Demonstração. Como $\frac{2 q-N-1}{N} \leq 2$ temos

$$
I_{0, n}^{q}=2 \int_{0}^{\frac{\pi}{2}} \cos ^{2 n-\frac{N-2 q+1}{N}} t d t \geq 2 \int_{0}^{\frac{\pi}{2}} \cos ^{2 n+2} t d t=I_{0, n+1},
$$

e a segunda desigualdade decorre do Fato A.0.2. 
Fato A.0.10 Seja $N \in \mathbb{N}^{*}$ e seja $C=\max _{1 \leq q \leq N} \frac{\Gamma\left(\frac{1}{2}\right) \Gamma\left(\frac{2 q-1}{2 N}\right)}{\Gamma\left(\frac{1}{2}+\frac{2 q-1}{2 N}\right)}$. Então $I_{0, n}^{q} \leq C$ e $I_{n, 0}^{q} \leq C$, $\forall n \geq 0, q=1,2, \cdots, N$.

Demonstração.

$$
\begin{aligned}
I_{0, n}^{q} & =\frac{\Gamma\left(\frac{1}{2}\right) \Gamma\left(n+\frac{2 q-1}{2 N}\right)}{\Gamma\left(n+\frac{1}{2}+\frac{2 q-1}{2 N}\right)} \\
& =\frac{\Gamma\left(\frac{1}{2}\right)\left(n-1+\frac{2 q-1}{2 N}\right)\left(n-2+\frac{2 q-1}{2 N}\right) \cdots\left(1+\frac{2 q-1}{2 N}\right) \frac{2 q-1}{2 N} \Gamma\left(\frac{2 q-1}{2 N}\right)}{\left(n+\frac{1}{2}-1+\frac{2 q-1}{2 N}\right)\left(n+\frac{1}{2}-2+\frac{2 q-1}{2 N}\right) \cdots\left(\frac{3}{2}+\frac{2 q-1}{2 N}\right)\left(\frac{1}{2}+\frac{2 q-1}{2 N}\right) \Gamma\left(\frac{1}{2}+\frac{2 q-1}{2 N}\right)} \\
& \leq \frac{\Gamma\left(\frac{1}{2}\right) \Gamma\left(\frac{2 q-1}{2 N}\right)}{\Gamma\left(\frac{1}{2}+\frac{2 q-1}{2 N}\right)} \leq C \\
I_{n, 0}^{q} & =\frac{\Gamma\left(n+\frac{1}{2}\right) \Gamma\left(\frac{2 q-1}{2 N}\right)}{\Gamma\left(n+\frac{1}{2}+\frac{2 q-1}{2 N}\right)} \\
& =\frac{\left(n-1+\frac{1}{2}\right)\left(n-2+\frac{1}{2}\right) \cdots\left(1+\frac{1}{2}\right) \frac{1}{2} \Gamma\left(\frac{1}{2}\right) \Gamma\left(\frac{2 q-1}{2 N}\right)}{\left(n-1+\frac{1}{2}+\frac{2 q-1}{2 N}\right)\left(n-2+\frac{1}{2}+\frac{2 q-1}{2 N}\right) \cdots\left(1+\frac{1}{2}+\frac{2 q-1}{2 N}\right)\left(\frac{1}{2}+\frac{2 q-1}{2 N}\right) \Gamma\left(\frac{1}{2}+\frac{2 q-1}{2 N}\right)} \\
\leq & \frac{\Gamma\left(\frac{1}{2}\right) \Gamma\left(\frac{2 q-1}{2 N}\right)}{\Gamma\left(\frac{1}{2}+\frac{2 q-1}{2 N}\right)} \leq C
\end{aligned}
$$

Fato A.0.11 Para $1 \leq q \leq N$ temos $\frac{I_{1,0}^{q}}{I_{0,1}^{q}}=\frac{N}{2 q-1}$.

Demonstração. De fato,

$$
\frac{I_{1,0}^{q}}{I_{0,1}^{q}}=\frac{\Gamma\left(\frac{3}{2}\right) \Gamma\left(\frac{2 q-1}{2 N}\right)}{\Gamma\left(1+\frac{N+2 q-1}{2 N}\right)} \frac{\Gamma\left(1+\frac{N+2 q-1}{2 N}\right)}{\Gamma\left(\frac{1}{2}\right) \Gamma\left(1+\frac{2 q-1}{2 N}\right)}=\frac{\frac{1}{2} \Gamma\left(\frac{1}{2}\right) \Gamma\left(\frac{2 q-1}{2 N}\right)}{\Gamma\left(\frac{1}{2}\right) \frac{2 q-1}{2 N} \Gamma\left(\frac{2 q-1}{2 N}\right)}=\frac{N}{2 q-1}
$$

Fato A.0.12 Se $1 \leq q \leq N$, então $\frac{I_{1,1}^{q}}{I_{0,2}^{q}} \leq \frac{1}{2} e \frac{I_{2,0}^{q}}{I_{0,2}^{q}} \leq \frac{3 N}{2(2 q-1)}$.

Demonstração. De fato, chamando $p=2 q-1$ temos

$$
\begin{aligned}
& \frac{I_{1,1}^{q}}{I_{0,2}^{q}}=\frac{\Gamma\left(\frac{3}{2}\right) \Gamma\left(1+\frac{p}{2 N}\right)}{\Gamma\left(\frac{5}{2}+\frac{p}{2 N}\right)} \frac{\Gamma\left(\frac{5}{2}+\frac{p}{2 N}\right)}{\Gamma\left(\frac{1}{2}\right) \Gamma\left(2+\frac{p}{2 N}\right)}=\frac{\frac{1}{2} \Gamma\left(\frac{1}{2}\right) \Gamma\left(1+\frac{p}{2 N}\right)}{\Gamma\left(\frac{1}{2}\right)\left(1+\frac{p}{2 N}\right) \Gamma\left(1+\frac{p}{2 N}\right)}=\frac{1}{2+\frac{p}{N}} \leq \frac{1}{2}, \\
& \frac{I_{2,0}^{q}}{I_{0,2}^{q}}=\frac{\Gamma\left(\frac{5}{2}\right) \Gamma\left(\frac{p}{2 N}\right)}{\Gamma\left(\frac{5}{2}+\frac{p}{2 N}\right)} \frac{\Gamma\left(\frac{5}{2}+\frac{p}{2 N}\right)}{\Gamma\left(\frac{1}{2}\right) \Gamma\left(2+\frac{p}{2 N}\right)}=\frac{\frac{3}{2} \frac{1}{2} \Gamma\left(\frac{1}{2}\right) \Gamma\left(\frac{p}{2 N}\right)}{\Gamma\left(\frac{1}{2}\right)\left(1+\frac{p}{2 N}\right) \frac{p}{2 N} \Gamma\left(\frac{p}{2 N}\right)}=\frac{3.1}{\left(2+\frac{p}{N}\right) \frac{p}{N}} \leq \frac{3 N}{2 p} .
\end{aligned}
$$


Fato A.0.13 Se $1 \leq q \leq N$, então $\frac{I_{1,2}^{q}}{I_{0,3}^{q}} \leq \frac{1}{4} e \frac{I_{2,1}^{q}}{I_{0,3}^{q}} \leq \frac{3}{8}$

Demonstração. De fato, chamando $p=2 q-1$ temos

$$
\begin{aligned}
\frac{I_{1,2}^{q}}{I_{0,3}^{q}} & =\frac{\Gamma\left(\frac{3}{2}\right) \Gamma\left(2+\frac{p}{2 N}\right)}{\Gamma\left(\frac{7}{2}+\frac{p}{2 N}\right)} \frac{\Gamma\left(\frac{7}{2}+\frac{p}{2 N}\right)}{\Gamma\left(\frac{1}{2}\right) \Gamma\left(3+\frac{p}{2 N}\right)}=\frac{\frac{1}{2} \Gamma\left(\frac{1}{2}\right) \Gamma\left(2+\frac{p}{2 N}\right)}{\Gamma\left(\frac{1}{2}\right)\left(2+\frac{p}{2 N}\right) \Gamma\left(2+\frac{p}{2 N}\right)}=\frac{1}{4+\frac{p}{N}} \leq \frac{1}{4}, \\
\frac{I_{2,1}^{q}}{I_{0,3}^{q}} & =\frac{\Gamma\left(\frac{5}{2}\right) \Gamma\left(1+\frac{p}{2 N}\right)}{\Gamma\left(\frac{7}{2}+\frac{p}{2 N}\right)} \frac{\Gamma\left(\frac{7}{2}+\frac{p}{2 N}\right)}{\Gamma\left(\frac{1}{2}\right) \Gamma\left(3+\frac{p}{2 N}\right)}=\frac{\frac{3}{2} \frac{1}{2} \Gamma\left(\frac{1}{2}\right) \Gamma\left(1+\frac{p}{2 N}\right)}{\Gamma\left(\frac{1}{2}\right)\left(2+\frac{p}{2 N}\right)\left(1+\frac{p}{2 N}\right) \Gamma\left(1+\frac{p}{2 N}\right)} \\
& =\frac{3.1}{\left(4+\frac{p}{N}\right)\left(2+\frac{p}{N}\right)} \leq \frac{3}{8} .
\end{aligned}
$$

Fato A.0.14 Se $1 \leq q \leq N$ e $2 \leq j \leq n-2$ então $\frac{I_{j, n-j}^{q}}{I_{1, n-1}^{q}} \leq \frac{3}{4}$.

Demonstração. De fato, seja $p=2 q-1$. Então, observando que $m \geq 3$ e $j \leq m-2$ temos

$$
\begin{aligned}
\frac{I_{j, n-j}^{q}}{I_{1, n-1}^{q}} & =\frac{\Gamma\left(j+\frac{1}{2}\right) \Gamma\left(n-j+\frac{p}{2 N}\right)}{\Gamma\left(\frac{3}{2}\right) \Gamma\left(n-1+\frac{p}{2 N}\right)} \\
& =\frac{\left(j-\frac{1}{2}\right)\left(j-\frac{3}{2}\right)\left(j-\frac{5}{2}\right) \cdots \frac{5}{2} \frac{3}{2} \Gamma\left(\frac{3}{2}\right) \Gamma\left(n-j+\frac{p}{2 N}\right)}{\Gamma\left(\frac{3}{2}\right)\left(n-2+\frac{p}{2 N}\right)\left(n-3+\frac{p}{2 N}\right) \cdots\left(n-j+1+\frac{p}{2 N}\right)\left(n-j+\frac{p}{2 N}\right) \Gamma\left(n-j+\frac{p}{2 N}\right)} \\
& \leq \frac{(2 j-1)(2 j-3)(2 j-5) \ldots 5.3}{(2 n-4)(2 n-6)(2 n-8) \cdots(2 n-(2 j-2))(2 n-2 j)} \leq \frac{3}{2(n-j)} \leq \frac{3}{4} .
\end{aligned}
$$

Fato A.0.15 Se $1 \leq q \leq N$ e $n \geq 4$ então $\frac{I_{n, 0}^{q}}{I_{0, n}^{q}} \leq \frac{15}{48} \frac{N}{(2 q-1)}(2 n-1)$

Demonstração. De fato, fazendo $p=2 q-1$ temos

$$
\begin{aligned}
\frac{I_{n, 0}^{q}}{I_{0, n}^{q}} & =\frac{\Gamma\left(n+\frac{1}{2}\right) \Gamma\left(\frac{p}{2 N}\right)}{\Gamma\left(\frac{1}{2}\right) \Gamma\left(n+\frac{p}{2 N}\right)} \\
& =\frac{\left(n-\frac{1}{2}\right)\left(n-\frac{3}{2}\right)\left(n-\frac{5}{2}\right) \ldots \frac{7}{2} \frac{5}{2} \frac{3}{2} \frac{1}{2} \Gamma\left(\frac{1}{2}\right) \Gamma\left(\frac{p}{2 N}\right)}{\Gamma\left(\frac{1}{2}\right)\left(n-1+\frac{p}{2 N}\right)\left(n-2+\frac{p}{2 N}\right)\left(n-3+\frac{p}{2 N}\right) \ldots\left(3+\frac{p}{2 N}\right)\left(2+\frac{p}{2 N}\right)\left(1+\frac{p}{2 N}\right) \frac{p}{2 N} \Gamma\left(\frac{p}{2 N}\right)} \\
& \leq \frac{(2 n-1)(2 n-3)(2 n-5) \ldots 7.5 .3 .1}{(2 n-2)(2 n-4)(2 n-6) \cdots(6)(4)(2) \frac{p}{N}} \leq \frac{(2 n-1) \cdot 5.3 .1}{6.4 .2 \cdot \frac{p}{N}}=\frac{15}{48} \frac{N}{p}(2 n-1)
\end{aligned}
$$

Fato A.0.16 Se $1 \leq q \leq N$ então $\frac{I_{3,0}^{q}}{I_{0,3}^{q}} \leq \frac{15 N}{8(2 q-1)}$ 
Demonstração. Análoga à do Fato A.0.15.

Fato A.0.17 Se $1 \leq q \leq N$ e $n \geq 1$ então $\frac{I_{n-1,1}^{q}}{I_{n, 0}^{q}}=\frac{p}{N(2 n-1)}$.

Demonstração. De fato, fazendo $p=2 q-1$ temos

$$
\frac{I_{n-1,1}^{q}}{I_{n, 0}^{q}}=\frac{\Gamma\left(n-\frac{1}{2}\right) \Gamma\left(1+\frac{p}{2 N}\right)}{\Gamma\left(n+\frac{1}{2}\right) \Gamma\left(\frac{p}{2 N}\right)}=\frac{\Gamma\left(n-\frac{1}{2}\right) \frac{p}{2 N} \Gamma\left(\frac{p}{2 N}\right)}{\left(n-\frac{1}{2}\right) \Gamma\left(n-\frac{1}{2}\right) \Gamma\left(\frac{p}{2 N}\right)}=\frac{p}{N(2 n-1)} .
$$

Fato A.0.18 Se $1 \leq q \leq N$ e $n \geq 2$ então $\frac{I_{1, n-1}^{q}}{I_{0, n}^{q}} \leq \frac{1}{(2 n-2)}$.

Demonstração. De fato, se $p=2 q-1$ então

$$
\frac{I_{1, n-1}^{q}}{I_{0, n}^{q}}=\frac{\Gamma\left(\frac{3}{2}\right) \Gamma\left(n-1+\frac{p}{2 N}\right)}{\Gamma\left(\frac{1}{2}\right) \Gamma\left(n+\frac{p}{2 N}\right)}=\frac{\frac{1}{2} \Gamma\left(\frac{1}{2}\right) \Gamma\left(n-1+\frac{p}{2 N}\right)}{\Gamma\left(\frac{1}{2}\right)\left(n-1+\frac{p}{2 N}\right) \Gamma\left(n-1+\frac{p}{2 N}\right)}=\frac{1}{2 n-2+\frac{p}{N}} \leq \frac{1}{2 n-2} .
$$

Fato A.0.19 Seja $B_{k}$ e $\widetilde{B}_{k}$ analíticas em zero com $k=1,2, \cdots, N$ tais que

$$
\frac{\widetilde{B}_{1}(h)}{\sqrt[2 N]{h^{2 N-1}}}+\frac{\widetilde{B}_{2}(h)}{\sqrt[2 N]{h^{2 N-3}}}+\ldots+\frac{\widetilde{B}_{N}(h)}{\sqrt[2 N]{h}}=\frac{B_{1}(h)}{\sqrt[2 N]{h^{2 N-1}}}+\frac{B_{2}(h)}{\sqrt[2 N]{h^{2 N-3}}}+\ldots+\frac{B_{N}(h)}{\sqrt[2 N]{h}},
$$

então $B_{k}=\widetilde{B}_{k}$

Demonstração. Seja

$$
U(h)=\left(\frac{\widetilde{B}_{1}(h)}{\sqrt[2 N]{h^{2 N-1}}}-\frac{B_{1}(h)}{\sqrt[2 N]{h^{2 N-1}}}\right)+\left(\frac{\widetilde{B}_{2}(h)}{\sqrt[2 N]{h^{2 N-3}}}-\frac{B_{2}(h)}{\sqrt[2 N]{h^{2 N-3}}}\right)+\ldots+\left(\frac{\widetilde{B}_{N}(h)}{\sqrt[2 N]{h}}-\frac{B_{N}(h)}{\sqrt[2 N]{h}}\right)=0
$$

Sejam

$$
\begin{aligned}
& B_{1}(h)=\sum_{k=1}^{\infty} b_{2 k+1} h^{k}, B_{2}(h)=\sum_{k=1}^{\infty} b_{2 k+2} h^{k}, \ldots, B_{N}(h)=\sum_{k=1}^{\infty} b_{2 k+N} h^{k} \\
& \widetilde{B}_{1}(h)=\sum_{k=1}^{\infty} \widetilde{b}_{2 k+1} h^{k}, \widetilde{B}_{2}(h)=\sum_{k=1}^{\infty} \widetilde{b}_{2 k+2} h^{k}, \ldots, \widetilde{B}_{N}(h)=\sum_{k=1}^{\infty} \widetilde{b}_{2 k+N} h^{k} \text { Sendo assim, }
\end{aligned}
$$




$$
\begin{aligned}
U\left(h^{2 N}\right) & =\left(\frac{1}{\left(h^{2 N}\right)^{\frac{2 N-1}{2 N}}} \sum_{k=1}^{\infty} \widetilde{b}_{2 k+1} h^{2 N k}-\frac{1}{\left(h^{2 N}\right)^{\frac{2 N-1}{2 N}}} \sum_{k=1}^{\infty} b_{2 k+1} h^{2 N k}\right) \\
& +\left(\frac{1}{\left(h^{2 N}\right)^{\frac{2 N-3}{2 N}}} \sum_{k=1}^{\infty} \widetilde{b}_{2 k+2} h^{2 N k}-\frac{1}{\left(h^{2 N}\right)^{\frac{2 N-3}{2 N}}} \sum_{k=1}^{\infty} b_{2 k+2} h^{2 N k}\right)+\ldots \\
& +\left(\frac{1}{\left(h^{2 N}\right)^{\frac{1}{2 N}}} \sum_{k=1}^{\infty} \widetilde{b}_{2 k+N} h^{2 N k}-\frac{1}{\left(h^{2 N}\right)^{\frac{1}{2 N}}} \sum_{k=1}^{\infty} b_{2 k+N} h^{2 N k}\right) \\
& =\left(\sum_{k=1}^{\infty} \widetilde{b}_{2 k+1} h^{2 N k-(1 N-1)}-\sum_{k=1}^{\infty} b_{2 k+1} h^{2 N k-(1 N-1)}\right) \\
& +\left(\sum_{k=1}^{\infty} \widetilde{b}_{2 k+2} h^{2 N k-(2 N-3)}-\sum_{k=1}^{\infty} b_{2 k+2} h^{2 N k-(2 N-3)}\right)+\ldots \\
& +\left(\sum_{k=1}^{\infty} \widetilde{b}_{2 k+N} h^{2 N k-1}-\sum_{k=1}^{\infty} b_{2 k+N} h^{2 N k-1}\right) \text { Seja, } u_{2 k+N}=\widetilde{b}_{2 k+N}-b_{2 k+N}, \text { assim, } \\
U\left(h^{2 N}\right)= & \left(\sum_{k=1}^{\infty} u_{2 k+1} h^{2 N k-2 N+1}+\sum_{k=1}^{\infty} u_{2 k+2} h^{2 N k-2 N+3}+\ldots+\sum_{k=1}^{\infty} u_{2 k+N} h^{2 N k-1}\right)=\sum_{j=0}^{\infty} u_{j} h^{j}=0 \\
\Leftrightarrow u_{j}=0 & \Leftrightarrow \widetilde{b}_{2 k+N}=b_{2 k+N} \Leftrightarrow B_{1}=\widetilde{B}_{1}, B_{2}=\widetilde{B}_{2}, \ldots, B_{N}=\widetilde{B}_{N} .
\end{aligned}
$$

Fato A.0.20 Seja $N \in \mathbb{N}^{*}$ e $1 \leq q \leq N$. Então

$$
I_{o, n}^{q} \geq \frac{I_{0,0}^{q}}{2 N(n+1)}, \forall n \in \mathbb{N} .
$$

Demonstração. $I_{0, n}^{q}=\frac{\Gamma\left(\frac{1}{2}\right) \Gamma\left(n+\frac{2 q-1}{2 N}\right)}{\Gamma\left(n+\frac{1}{2}+\frac{2 q-1}{2 N}\right)}$.

Se $n=0$ a desigualdade é imediata.

Se $n \geq 1$ temos

$$
\begin{aligned}
I_{0, n}^{q} & =\frac{\Gamma\left(\frac{1}{2}\right)\left(n-1+\frac{2 q-1}{2 N}\right)\left(n-2+\frac{2 q-1}{2 N}\right) \cdots\left(\frac{2 q-1}{2 N}\right) \Gamma\left(\frac{2 q-1}{2 N}\right)}{\left(n-1+\frac{1}{2}+\frac{2 q-1}{2 N}\right)\left(n-2+\frac{1}{2}+\frac{2 q-1}{2 N}\right) \cdots\left(\frac{1}{2}+\frac{2 q-1}{2 N}\right) \Gamma\left(\frac{1}{2}+\frac{2 q-1}{2 N}\right)} \\
& \geq \frac{\frac{2 q-1}{2 N} I_{0,0}^{q}}{\left(n-1+\frac{1}{2}+\frac{2 q-1}{2 N}\right)} \geq \frac{I_{0,0}^{q}}{2 N(n+1)}
\end{aligned}
$$

Observação: Nos Fatos de A.0.21 a $\Lambda .0 .26$, abaixo usaremos a seguinte 
Definição A.0.3

$$
\begin{aligned}
& \widetilde{I}_{i, j}=2 \int_{0}^{\frac{\pi}{2}} \operatorname{sen}^{\left(2 i-\frac{1}{2}\right)} \theta \cos ^{2 j} \theta d \theta=\beta\left(i+\frac{1}{4}, j+\frac{1}{2}\right)=\frac{\Gamma\left(i+\frac{1}{4}\right) \Gamma\left(j+\frac{1}{2}\right)}{\Gamma\left(i+j+\frac{3}{4}\right)} \\
& \widehat{I}_{i, j}=2 \int_{0}^{\frac{\pi}{2}} \operatorname{sen}^{\left(2 i+\frac{1}{2}\right)} \theta \cos ^{2 j} \theta d \theta=\beta\left(i+\frac{3}{4}, j+\frac{1}{2}\right)=\frac{\Gamma\left(i+\frac{3}{4}\right) \Gamma\left(j+\frac{1}{2}\right)}{\Gamma\left(i+j+\frac{5}{4}\right)}
\end{aligned}
$$

Note que $\widetilde{I}_{i, j}=I_{j, i}^{1}$ e $\widehat{I}_{i, j}=I_{j, i}^{2}$ onde $I_{j, i}^{q}$ são dadas pela definição A.0.2 com $N=2$.

Fato A.0.21 $\widetilde{I}_{0,0}=\frac{\Gamma\left(\frac{1}{4}\right) \Gamma\left(\frac{1}{2}\right)}{\Gamma\left(\frac{3}{4}\right)}=\frac{\Gamma\left(\frac{1}{4}\right) \sqrt{\pi}}{\Gamma\left(\frac{3}{4}\right)}$

Fato A.0.22 $\widetilde{I}_{0,1}=\frac{\Gamma\left(\frac{1}{4}\right) \Gamma\left(1+\frac{1}{2}\right)}{\Gamma\left(1+\frac{3}{4}\right)}=\frac{\Gamma\left(\frac{1}{4}\right) \frac{1}{2} \sqrt{\pi}}{\frac{3}{4} \Gamma\left(\frac{3}{4}\right)}$

Fato A.0.23 Se $1 \leq j \leq n-1$ então $\frac{\widetilde{I}_{j, n-j}}{\widetilde{I}_{1, n-1}} \leq 1$.

Demonstração. De fato,

$$
\begin{aligned}
\frac{\widetilde{I}_{j, n-j}}{\widetilde{I}_{1, n-1}} & =\frac{\Gamma\left(j+\frac{1}{4}\right) \Gamma\left(n-j+\frac{1}{2}\right)}{\Gamma\left(1+\frac{1}{4}\right) \Gamma\left(n-\frac{1}{2}\right)} \\
& =\frac{\left(j-\frac{3}{4}\right)\left(j-\frac{7}{4}\right)\left(j-\frac{11}{4}\right) \cdots \frac{9}{4} \frac{5}{4} \Gamma\left(\frac{5}{4}\right) \Gamma\left(n-j+\frac{1}{2}\right)}{\Gamma\left(\frac{5}{4}\right)\left(n-\frac{3}{2}\right)\left(n-\frac{5}{2}\right)\left(n-\frac{7}{2}\right) \cdots\left(n-j+\frac{1}{2}\right) \Gamma\left(n-j+\frac{1}{2}\right)} \\
& =\frac{(4 j-3)(4 j-7)(4 j-11) \cdots 9.5}{(4 n-6)(4 n-10)(4 n-14) \cdots(4(n-j)+2)} \leq 1
\end{aligned}
$$

Fato A.0.24 Para todo $n \in \mathbb{N}$ temos $\frac{\widetilde{I}_{n, 0}}{\widetilde{I}_{0, n}} \leq 1$.

Demonstração. De fato,

$$
\begin{aligned}
\frac{\widetilde{I}_{n, 0}}{\widetilde{I}_{0, n}} & =\frac{\Gamma\left(n+\frac{1}{4}\right) \Gamma\left(\frac{1}{2}\right)}{\Gamma\left(n+\frac{3}{4}\right)} \frac{\Gamma\left(n+\frac{3}{4}\right)}{\Gamma\left(\frac{1}{4}\right) \Gamma\left(n+\frac{1}{2}\right)}=\frac{\left(n-\frac{3}{4}\right)\left(n-\frac{7}{4}\right)\left(n-\frac{11}{4}\right) \cdots \frac{5}{4} \frac{1}{4} \Gamma\left(\frac{1}{4}\right) \Gamma\left(\frac{1}{2}\right)}{\Gamma\left(\frac{1}{4}\right)\left(n-\frac{1}{2}\right)\left(n-\frac{3}{2}\right)\left(n-\frac{5}{2}\right) \cdots \frac{3}{2} \frac{1}{2} \Gamma\left(\frac{1}{2}\right)} \\
& =\frac{\frac{1}{2^{n}}\left(2 n-\frac{3}{2}\right)\left(2 n-\frac{7}{2}\right)\left(2 n-\frac{11}{2}\right) \cdots \frac{5}{2} \frac{1}{2}}{\frac{1}{2^{n}}(2 n-1)(2 n-3)(2 n-5) \cdots 3.1}<1
\end{aligned}
$$


Fato A.0.25 Para todo $n \in \mathbb{N}^{*}$ temos $\frac{\widetilde{I}_{1, n-1}}{\widetilde{I}_{0, n}}<\frac{1}{2(2 n-1)}$. Além disso, vale $\frac{(n+2)(n-1)}{2} \frac{\widetilde{I}_{1, n-1}}{\widetilde{I}_{0, n}} \leq \frac{n}{4}$

Demonstração.

$$
\frac{\widetilde{I}_{1, n-1}}{\widetilde{I}_{0, n}}=\frac{\Gamma\left(\frac{5}{4}\right) \Gamma\left(n-\frac{1}{2}\right)}{\Gamma\left(n+\frac{3}{4}\right)} \frac{\Gamma\left(n+\frac{3}{4}\right)}{\Gamma\left(\frac{1}{4}\right) \Gamma\left(n+\frac{1}{2}\right)}=\frac{\frac{1}{4} \Gamma\left(\frac{1}{4}\right) \Gamma\left(n-\frac{1}{2}\right)}{\Gamma\left(\frac{1}{4}\right)\left(n-\frac{1}{2}\right) \Gamma\left(n-\frac{1}{2}\right)}=\frac{1}{4} \frac{2}{2 n-1}=\frac{1}{2(2 n-1)}
$$

Além disso,

$$
\frac{(n+2)(n-1)}{2} \frac{I_{1, n-1}^{1}}{I_{0, n}^{1}}=\frac{(n+2)(n-1)}{4(2 n-1)}=\frac{(n+2)(n-1)}{8\left(n-\frac{1}{2}\right)} \leq \frac{n+2}{8} \leq \frac{n}{4}
$$

Fato A.0.26 Se $n \in \mathbb{N}^{*}$ temos $\frac{\Gamma\left(\frac{1}{4}\right) \sqrt{\pi}}{3 \Gamma\left(\frac{3}{4}\right) \widetilde{I}_{0, n}}<\frac{3 n}{4}$.

Demonstração.

$$
\begin{aligned}
\frac{\Gamma\left(\frac{1}{4}\right) \sqrt{\pi}}{3 \Gamma\left(\frac{3}{4}\right) \widetilde{I}_{0, n}} & =\frac{\Gamma\left(\frac{1}{4}\right) \sqrt{\pi}}{3 \Gamma\left(\frac{3}{4}\right)} \frac{\Gamma\left(n+\frac{3}{4}\right)}{\Gamma\left(\frac{1}{4}\right) \Gamma\left(n+\frac{1}{2}\right)}=\frac{\left(n-\frac{1}{4}\right)\left(n-\frac{5}{4}\right) \cdots \frac{7}{4} \frac{3}{4} \Gamma\left(\frac{3}{4}\right) \sqrt{\pi}}{3 \Gamma\left(\frac{3}{4}\right)\left(n-\frac{1}{2}\right)\left(n-\frac{3}{2}\right) \cdots \frac{3}{2} \frac{1}{2} \Gamma\left(\frac{1}{2}\right)} \\
& =\frac{(4 n-1)(4 n-5)(4 n-9) \cdots 11.7 .3}{3(4 n-2)(4 n-6) \cdots 10.6 .2} \\
& <\frac{1}{6}(4 n-1)=\frac{8 n-2}{12}<\frac{3 n}{4}
\end{aligned}
$$

Observação: Nos Fatos de A.0.30 a A.0.38, abaixo usaremos a seguinte

\section{Definição A.0.4}

$$
\begin{aligned}
& \widetilde{I}_{i, j}^{1}=2 \int_{0}^{\frac{\pi}{2}} \operatorname{sen}^{\left(2 i-\frac{1}{2}\right)} \theta \cos ^{\left(2 j-\frac{1}{2}\right)} \theta d \theta=\beta\left(i+\frac{1}{4}, j+\frac{1}{4}\right)=\frac{\Gamma\left(i+\frac{1}{4}\right) \Gamma\left(j+\frac{1}{4}\right)}{\Gamma\left(i+j+\frac{1}{2}\right)} \\
& \widetilde{I}_{i, j}^{2}=2 \int_{0}^{\frac{\pi}{2}} \operatorname{sen}^{\left(2 i-\frac{1}{2}\right)} \theta \cos ^{\left(2 j+\frac{1}{2}\right)} \theta d \theta=\beta\left(i+\frac{1}{4}, j+\frac{3}{4}\right)=\frac{\Gamma\left(i+\frac{1}{4}\right) \Gamma\left(j+\frac{3}{4}\right)}{\Gamma(i+j+1)} \\
& \widetilde{I}_{i, j}^{3}=2 \int_{0}^{\frac{\pi}{2}} \operatorname{sen}^{\left(2 i+\frac{1}{2}\right)} \theta \cos ^{\left(2 j+\frac{1}{2}\right)} \theta d \theta=\beta\left(i+\frac{3}{4}, j+\frac{3}{4}\right)=\frac{\Gamma\left(i+\frac{3}{4}\right) \Gamma\left(j+\frac{3}{4}\right)}{\Gamma\left(i+j+\frac{3}{2}\right)} \\
& \widetilde{I}_{i, j}^{4}=2 \int_{0}^{\frac{\pi}{2}} \operatorname{sen}^{\left(2 i+\frac{1}{2}\right)} \theta \cos ^{\left(2 j-\frac{1}{2}\right)} \theta d \theta=\beta\left(i+\frac{3}{4}, j+\frac{1}{4}\right)=\frac{\Gamma\left(i+\frac{3}{4}\right) \Gamma\left(j+\frac{1}{4}\right)}{\Gamma(i+j+1)}
\end{aligned}
$$


Fato A.0.27 Para $n \in \mathbb{N}^{*}$, temos

$$
\widetilde{I}_{0, n}^{1} \geq \frac{\left(\Gamma\left(\frac{1}{4}\right)\right)^{2}}{(4 n-2) \sqrt{\pi}} e I_{0, n}^{2} \geq \frac{3 \Gamma\left(\frac{1}{4}\right) \Gamma\left(\frac{3}{4}\right)}{4 n}
$$

Demonstração. De fato, pela definição A.0.4 temos

$$
\begin{aligned}
\widetilde{I}_{0, n}^{1} & =\frac{\Gamma\left(\frac{1}{4}\right)\left(n-\frac{3}{4}\right)\left(n-\frac{7}{4}\right) \cdots \frac{5}{4} \frac{1}{4} \Gamma\left(\frac{1}{4}\right)}{\left(n-\frac{1}{2}\right)\left(n-\frac{3}{2}\right) \cdots \frac{3}{2} \frac{1}{2} \Gamma\left(\frac{1}{2}\right)} \\
& =\frac{\left(\Gamma\left(\frac{1}{4}\right)\right)^{2}(4 n-3)(4 n-7) \cdots 5.1}{(4 n-2)(4 n-6) \cdots 6.2 \Gamma\left(\frac{1}{2}\right)} \geq \frac{\left(\Gamma\left(\frac{1}{4}\right)\right)^{2}}{(4 n-2) \sqrt{\pi}} \\
\widetilde{I}_{0, n}^{2} & =\frac{\Gamma\left(\frac{1}{4}\right)\left(n-\frac{1}{4}\right)\left(n-\frac{5}{4}\right) \cdots \frac{7}{4} \frac{3}{4} \Gamma\left(\frac{3}{4}\right)}{n \cdot(n-1)(n-2) \cdots 2.1} \\
& =\frac{\Gamma\left(\frac{1}{4}\right) \Gamma\left(\frac{3}{4}\right)(4 n-1)(4 n-5) \cdots 7.3}{4 n \cdot(4 n-4)(4 n-8) \cdots 8.4} \geq \frac{3 \Gamma\left(\frac{1}{4}\right) \Gamma\left(\frac{3}{4}\right)}{4 n}
\end{aligned}
$$

Fato A.0.28 $\widetilde{I}_{i, j}^{2}=\widetilde{I}_{j, i}^{4}$.

Demonstração.

$$
\begin{aligned}
\widetilde{I}_{i, j}^{2} & =2 \int_{0}^{\frac{\pi}{2}} \operatorname{sen}^{\left(2 i-\frac{1}{2}\right)} \theta \cos ^{\left(2 j+\frac{1}{2}\right)} \theta d \theta=\beta\left(i+\frac{1}{4}, j+\frac{3}{4}\right)=\frac{\Gamma\left(i+\frac{1}{4}\right) \Gamma\left(j+\frac{3}{4}\right)}{\Gamma(i+j+1)} \\
& =\frac{\Gamma\left(j+\frac{3}{4}\right) \Gamma\left(i+\frac{1}{4}\right)}{\Gamma(i+j+1)}=\beta\left(j+\frac{3}{4}, i+\frac{1}{4}\right)=2 \int_{0}^{\frac{\pi}{2}} \operatorname{sen}^{\left(2 j+\frac{1}{2}\right)} \theta \cos ^{\left(2 i-\frac{1}{2}\right)} \theta d \theta=\widetilde{I}_{j, i}^{4}
\end{aligned}
$$

Fato A.0.29 $\widetilde{I}_{i, j}^{1}, \widetilde{I}_{i, j}^{2}, \widetilde{I}_{i, j}^{3}$ satisfazem: (a) $\widetilde{I}_{i, j}^{1}=\widetilde{I}_{j, i}^{1}$, (b) $\widetilde{I}_{i, j}^{3}=\widetilde{I}_{j, i}^{3}$ e $(c) \widetilde{I}_{n-1,1}^{2} \leq I_{1, n-1}^{2}$

Demonstração.
(a) $\widetilde{I}_{i, j}^{1}=2 \int_{0}^{\frac{\pi}{2}} \operatorname{sen}^{\left(2 i-\frac{1}{2}\right)} \theta \cos ^{\left(2 j-\frac{1}{2}\right)} \theta d \theta=\frac{\Gamma\left(i+\frac{1}{4}\right) \Gamma\left(j+\frac{1}{4}\right)}{\Gamma\left(i+j+\frac{1}{2}\right)} \Rightarrow \widetilde{I}_{i, j}^{1}=\widetilde{I}_{j, i}^{1}$
(b) $\widetilde{I}_{i, j}^{3}=2 \int_{0}^{\frac{\pi}{2}} \operatorname{sen}^{\left(2 i+\frac{1}{2}\right)} \theta \cos ^{\left(2 j+\frac{1}{2}\right)} \theta d \theta=\frac{\Gamma\left(i+\frac{3}{4}\right) \Gamma\left(j+\frac{3}{4}\right)}{\Gamma\left(i+j+\frac{3}{2}\right)} \Rightarrow \widetilde{I}_{i, j}^{3}=\widetilde{I}_{j, i}^{3}$

De fato,
(c) $\widetilde{I}_{i, j}^{2}=2 \int_{0}^{\frac{\pi}{2}} \operatorname{sen}^{\left(2 i-\frac{1}{2}\right)} \theta \cos ^{\left(2 j+\frac{1}{2}\right)} \theta d \theta=\frac{\Gamma\left(i+\frac{1}{4}\right) \Gamma\left(j+\frac{3}{4}\right)}{\Gamma(i+j+1)} \Rightarrow \widetilde{I}_{n-1,1}^{2} \leq I_{1, n-1}^{2}$, 
pois

$$
\frac{\widetilde{I}_{n-1,1}^{2}}{\widetilde{I}_{1, n-1}^{2}}=\frac{\Gamma\left(n-\frac{3}{4}\right) \Gamma\left(\frac{7}{4}\right)}{\Gamma(n+1)} \frac{\Gamma(n+1)}{\Gamma\left(\frac{5}{4}\right) \Gamma\left(n-\frac{1}{4}\right)}=\frac{\left(n-\frac{7}{4}\right)\left(n-\frac{11}{4}\right) \cdots \frac{9}{4} \frac{5}{4} \Gamma\left(\frac{5}{4}\right) \Gamma\left(\frac{7}{4}\right)}{\Gamma\left(\frac{5}{4}\right)\left(n-\frac{5}{4}\right)\left(n-\frac{9}{4}\right) \cdots \frac{11}{4} \frac{7}{4} \Gamma\left(\frac{7}{4}\right)} \leq 1
$$

Fato A.0.30 Se $0<j \leq n-1$, entãa $\frac{\widetilde{I}_{j, n-j}^{1}}{\widetilde{I}_{0, n}^{1}} \leq 1$

Demonstração.

$$
\begin{aligned}
\frac{\widetilde{I}_{j, n-j}^{1}}{\widetilde{I}_{0, n}^{1}} & =\frac{\Gamma\left(j+\frac{1}{4}\right) \Gamma\left(n-j+\frac{1}{4}\right)}{\Gamma\left(\frac{1}{4}\right) \Gamma\left(n+\frac{1}{4}\right)} \\
& =\frac{\left(j-\frac{3}{4}\right)\left(j-\frac{7}{4}\right)\left(j-\frac{11}{4}\right) \cdots \frac{5}{4} \frac{1}{4} \Gamma\left(\frac{1}{4}\right) \Gamma\left(n-j+\frac{1}{4}\right)}{\Gamma\left(\frac{1}{4}\right)\left(n-\frac{3}{4}\right)\left(n-\frac{7}{4}\right)\left(n-\frac{11}{4}\right) \cdots\left(n-j+\frac{5}{4}\right)\left(n-j+\frac{1}{4}\right) \Gamma\left(n-j+\frac{1}{4}\right)} \\
& =\frac{(4 j-3)(4 j-7)(4 j-11) \cdots 5.1}{(4 n-3)(4 n-7)(4 n-11) \cdots(4(n-j)+5)(4(n-j)+1)} \leq 1 .
\end{aligned}
$$

Fato A.0.31 Se $0 \leq i \leq n-2$, então $\frac{\widetilde{I}_{n-i-1, i}^{3}}{\widetilde{I}_{0, n-1}^{3}} \leq 1$.

Demonstração. De fato,

$$
\begin{aligned}
\frac{\widetilde{I}_{n-i-1, i}^{3}}{\widetilde{I}_{0, n-1}^{3}} & =\frac{\Gamma\left(n-i-1+\frac{3}{4}\right) \Gamma\left(i+\frac{3}{4}\right)}{\Gamma\left(\frac{3}{4}\right) \Gamma\left(n-\frac{1}{4}\right)} \\
& =\frac{\left(n-i-1-\frac{1}{4}\right)\left(n-i-1-\frac{5}{4}\right) \cdots \frac{7}{4} \frac{3}{4} \Gamma\left(\frac{3}{4}\right) \Gamma\left(i+\frac{3}{4}\right)}{\Gamma\left(\frac{3}{4}\right)\left(n-\frac{5}{4}\right)\left(n-\frac{9}{4}\right) \cdots\left(i+1+\frac{3}{4}\right)\left(i+1-1+\frac{3}{4}\right) \Gamma\left(i+1-1+\frac{3}{4}\right)} \\
& =\frac{(4 n-4 i-5)(4 n-4 i-9) \cdots 7.3}{(4 n-5)(4 n-9) \cdots(4 i+7)(4 i+3)} \leq 1
\end{aligned}
$$

Fato A.0.32 Se $n \geq 2$, então $\frac{\widetilde{I}_{0, n-1}^{3}}{\widetilde{I}_{0, n}^{1}} \leq \frac{2}{3}$

Demonstração. De fato,

$$
\begin{aligned}
\frac{\widetilde{I}_{0, n-1}^{3}}{\widetilde{I}_{0, n}^{1}} & =\frac{\Gamma\left(\frac{3}{4}\right) \Gamma\left(n-\frac{1}{4}\right)}{\Gamma\left(n+\frac{1}{2}\right)} \frac{\Gamma\left(n+\frac{1}{2}\right)}{\Gamma\left(\frac{1}{4}\right) \Gamma\left(n+\frac{1}{4}\right)}=\frac{\left[\Gamma\left(\frac{3}{4}\right)\right]^{2}\left(n-\frac{5}{4}\right)\left(n-\frac{9}{4}\right) \ldots \frac{73}{4} \frac{3}{4}}{\left[\Gamma\left(\frac{1}{4}\right)\right]^{2}\left(n-\frac{3}{4}\right)\left(n-\frac{7}{4}\right) \ldots \frac{5}{4} \frac{1}{4}} \\
& =\frac{\left[\Gamma\left(\frac{3}{4}\right)\right]^{2}}{\frac{1}{4}\left[\Gamma\left(\frac{1}{4}\right)\right]^{2}} \frac{(4 n-5)(4 n-9) \ldots 7.3}{(4 n-3)(4 n-7) \ldots 9.5} \leq \frac{2}{3} .
\end{aligned}
$$


Fato A.0.33 Se $0 \leq i \leq n-1$, então $\frac{\widetilde{I}_{n-i, i}^{2}}{\widetilde{I}_{0, n}^{2}}<1$

Demonstração. De fato,

$$
\begin{aligned}
\frac{\widetilde{I}_{n-i, i}^{2}}{\widetilde{I}_{0, n}^{2}} & =\frac{\Gamma\left(n-i+\frac{1}{4}\right) \Gamma\left(i+\frac{3}{4}\right)}{\Gamma\left(\frac{1}{4}\right) \Gamma\left(n+\frac{3}{4}\right)}=\frac{\left(n-i-\frac{3}{4}\right)\left(n-i-\frac{7}{4}\right) \cdots \frac{5}{4} \frac{1}{4} \Gamma\left(\frac{1}{4}\right) \Gamma\left(i+\frac{3}{4}\right)}{\Gamma\left(\frac{1}{4}\right)\left(n-\frac{1}{4}\right)\left(n-\frac{5}{4}\right) \cdots\left(i+\frac{7}{4}\right)\left(i+\frac{3}{4}\right) \Gamma\left(i+\frac{3}{4}\right)} \\
& =\frac{(4 n-(4 i+3))(4 n-(4 i+7))(4 n-(4 i+11)) \cdots 5.1}{(4 n-1)(4 n-5)(4 n-9) \cdots(4 i+7)(4 i+3)}<1
\end{aligned}
$$

Usaremos nas demonstrações de $(b)$ e $(d)$ no Fato A.0.35, o seguinte resultado:

Fato A.0.34

$$
\frac{\widetilde{I}_{0,0}^{3}}{\widetilde{I}_{0,1}^{1}}=\frac{4\left[\Gamma\left(\frac{3}{4}\right)\right]^{2}}{\left[\Gamma\left(\frac{1}{4}\right)\right]^{2}}=2 \frac{\widetilde{I}_{0,0}^{3}}{\widetilde{I}_{0,0}^{1}} \leq 2
$$

Demonstração. De fato,

$$
2 \frac{\widetilde{I}_{0,0}^{3}}{\widetilde{I}_{0,0}^{1}}=\frac{2\left[\Gamma\left(\frac{3}{4}\right)\right]^{2}}{\Gamma\left(\frac{3}{4}\right)} \frac{\Gamma\left(\frac{1}{4}\right)}{\left[\Gamma\left(\frac{1}{4}\right)\right]^{2}}=\frac{4\left[\Gamma\left(\frac{3}{4}\right)\right]^{2}}{\left[\Gamma\left(\frac{1}{4}\right)\right]^{2}}=\frac{\left[\Gamma\left(\frac{3}{4}\right)\right]^{2}}{\Gamma\left(\frac{3}{4}\right)} \frac{\Gamma\left(\frac{3}{4}\right)}{\Gamma\left(\frac{1}{4}\right) \Gamma\left(\frac{5}{4}\right)}=\frac{\widetilde{I}_{0,0}^{3}}{\widetilde{I}_{0,1}^{1}} .
$$

Por outro lado, temos

$$
\begin{aligned}
\frac{\widetilde{I}_{0,0}^{3}}{2} & =\int_{0}^{\frac{\pi}{2}} \operatorname{sen}^{\frac{1}{2}} \theta \cos ^{\frac{1}{2}} \theta d \theta \leq\left(\int_{0}^{\frac{\pi}{2}}\left(\operatorname{sen}^{\frac{1}{2}} \theta\right)^{2} d \theta\right)^{\frac{1}{2}}\left(\int_{0}^{\frac{\pi}{2}}\left(\cos ^{\frac{1}{2}} \theta\right)^{2} d \theta\right)^{\frac{1}{2}} \\
& =\left(\int_{0}^{\frac{\pi}{2}} \operatorname{sen} \theta d \theta\right)^{\frac{1}{2}}\left(\int_{0}^{\frac{\pi}{2}} \cos \theta d \theta\right)^{\frac{1}{2}}=1 \leq \frac{\pi}{2} \leq \int_{0}^{\frac{\pi}{2}} \operatorname{sen}^{-\frac{1}{2}} \theta \cos ^{-\frac{1}{2}} \theta d \theta=\frac{\widetilde{I}_{0,0}^{1}}{2}
\end{aligned}
$$

donde segue que $\frac{\widetilde{I}_{0,0}^{3}}{\widetilde{I}_{0,1}^{1}} \leq 1$.

Fato A.0.35 Para $n \geq 2$, temos

$$
\begin{aligned}
& \text { (a) } \frac{\mathcal{A}}{n} \leq \widetilde{I}_{0, n}^{1}, \text { onde } \mathcal{A}=\frac{\left[\Gamma\left(\frac{1}{4}\right)\right]^{2}}{4 \Gamma\left(\frac{1}{2}\right)} \\
& \text { (b) } \frac{\widetilde{I}_{0, n-1}^{3}}{\widetilde{I}_{0, n}^{1}} \leq 2 \\
& \text { (c) } \frac{n-1}{2} \cdot \frac{I_{1, n-1}^{1}}{I_{0, n}^{1}}<\frac{1}{8} \\
& \text { (d) } \frac{n-2}{2} \cdot \frac{\widetilde{I}_{1, n-2}^{3}}{\widetilde{I}_{0, n}^{1}}<\frac{1}{8} n \geq 3
\end{aligned}
$$


Demonstração. De fato,

$$
\begin{aligned}
& \text { (a) } \quad \widetilde{I}_{0, n}^{1}=\frac{\Gamma\left(\frac{1}{4}\right) \Gamma\left(n+\frac{1}{4}\right)}{\Gamma\left(n+\frac{1}{2}\right)} \\
& =\frac{\Gamma\left(\frac{1}{4}\right)(4 n-3)(4 n-7) \ldots 5.1 \Gamma\left(\frac{1}{4}\right) 4^{-n}}{2^{-n}(2 n-1)(2 n-3) \ldots 3.1 \Gamma\left(\frac{1}{2}\right)}=\frac{\Gamma\left(\frac{1}{4}\right) \Gamma\left(\frac{1}{4}\right)}{\Gamma\left(\frac{1}{2}\right)} \frac{(4 n-3)(4 n-7) \ldots 5.1}{(4 n-2)(4 n-6) \ldots 6.2} \\
& \geq \frac{\Gamma\left(\frac{1}{4}\right) \Gamma\left(\frac{1}{4}\right)}{\Gamma\left(\frac{1}{2}\right)} \frac{1}{4 n-2}=\frac{\Gamma\left(\frac{1}{4}\right) \Gamma\left(\frac{1}{4}\right)}{4 \Gamma\left(\frac{1}{2}\right)} \frac{1}{n-\frac{1}{2}}=\frac{\mathcal{A}}{n-\frac{1}{2}} \geq \frac{\mathcal{A}}{n} \\
& \text { (b) } \frac{\widetilde{I}_{0, n-1}^{3}}{\widetilde{I}_{0, n}^{1}}=\frac{\Gamma\left(\frac{3}{4}\right) \Gamma\left(n-\frac{1}{4}\right)}{\Gamma\left(n+\frac{1}{2}\right)} \frac{\Gamma\left(n+\frac{1}{2}\right)}{\Gamma\left(\frac{1}{4}\right) \Gamma\left(n+\frac{1}{4}\right)}=\frac{\left[\Gamma\left(\frac{3}{4}\right)\right]^{2}\left(n-\frac{5}{4}\right)\left(n-\frac{9}{4}\right) \ldots \frac{7}{4} \frac{3}{4}}{\left[\Gamma\left(\frac{1}{4}\right)\right]^{2}\left(n-\frac{3}{4}\right)\left(n-\frac{7}{4}\right) \ldots \frac{5}{4} \frac{1}{4}} \\
& =\frac{\left[\Gamma\left(\frac{3}{4}\right)\right]^{2}}{\frac{1}{4}\left[\Gamma\left(\frac{1}{4}\right)\right]^{2}} \frac{(4 n-5)(4 n-9) \ldots 7.3}{(4 n-3)(4 n-7) \ldots 9.5} \leq 2 \text {, pelo Fato (A.0.34). } \\
& \text { (c) } \frac{\widetilde{I}_{1, n-1}^{1}}{\widetilde{I}_{0, n}^{1}}=\frac{\frac{1}{4} \Gamma\left(\frac{1}{4}\right) \Gamma\left(n-\frac{3}{4}\right)}{\Gamma\left(n+\frac{1}{2}\right)} \frac{\Gamma\left(n+\frac{1}{2}\right)}{\Gamma\left(\frac{1}{4}\right) \Gamma\left(n+\frac{1}{4}\right)}=\frac{\Gamma\left(n-\frac{3}{4}\right)}{4 \Gamma\left(n+\frac{1}{4}\right)}=\frac{\Gamma\left(n-\frac{3}{4}\right)}{4\left(n-\frac{3}{4} \Gamma\left(n-\frac{3}{4}\right)\right.}=\frac{1}{4 n-3} \\
& =\frac{1}{4\left(n-\frac{3}{4}\right)} \text { Com isso, } \frac{n-1}{2} \cdot \frac{\widetilde{I}_{1, n-1}^{1}}{\widetilde{I}_{0, n}^{1}}=\frac{1}{8} \frac{n-1}{n-\frac{3}{4}}<\frac{1}{8} \text {. }
\end{aligned}
$$

Usando o Fato (A.0.34), temos

$$
\text { (d) } \begin{aligned}
\frac{\widetilde{I}_{1, n-2}^{3}}{\widetilde{I}_{0, n}^{1}} & =\frac{\Gamma\left(\frac{7}{4}\right) \Gamma\left(n-\frac{5}{4}\right)}{\Gamma\left(n+\frac{1}{2}\right)} \frac{\Gamma\left(n+\frac{1}{2}\right)}{\Gamma\left(\frac{1}{4}\right) \Gamma\left(n+\frac{1}{4}\right)}=\frac{\frac{3}{4}\left[\Gamma\left(\frac{3}{4}\right)\right]^{2}\left(n-\frac{9}{4}\right)\left(n-\frac{13}{4}\right) \ldots \frac{7}{4} \frac{3}{4}}{\left[\Gamma\left(\frac{1}{4}\right)\right]^{2}\left(n-\frac{3}{4}\right)\left(n-\frac{7}{4}\right) \ldots \frac{5}{4} \frac{1}{4}} \\
& =\frac{\left[\Gamma\left(\frac{3}{4}\right)\right]^{2}}{\frac{1}{4}\left[\Gamma\left(\frac{1}{4} \frac{1}{4}\right)\right]^{2}} \frac{(4 n-9)(4 n-13) \ldots 11.7 .3 .3}{(4 n-3)(4 n-7) \ldots 13.9 .5} \\
& =\frac{\frac{6}{4}\left[\Gamma\left(\frac{3}{4}\right)\right]^{2}}{\frac{1}{4}\left[\Gamma\left(\frac{1}{4}\right)\right]^{2}} \frac{1}{4 n-3} \frac{(4 n-9)(4 n-13) \ldots 11 . \frac{28}{6} \cdot 9}{(4 n-7)(4 n-11) \ldots 13.5 .9} \leq \frac{3}{(4 n-3)} \\
& <\frac{3}{4\left(n-\frac{3}{4}\right)}, \text { Com isso, } \frac{n-2}{2} \cdot \frac{\widetilde{I}_{1, n-2}^{3}}{\widetilde{I}_{0, n}^{1}}<\frac{3}{8} \frac{n-2}{n-\frac{3}{4}}<\frac{3}{8} .
\end{aligned}
$$

Fato A.0.36 Para $n \geq 2$ temos
(a) $\frac{n-1}{2} \frac{\widetilde{I}_{1, n-1}^{2}}{\widetilde{I}_{0, n}^{2}}=\frac{n-1}{n-\frac{1}{4}} \frac{1}{8}<\frac{1}{8}$.
(b) $\frac{\beta}{n} \leq \widetilde{I}_{0, n}^{2}$ onde $\beta=\frac{3}{4} \frac{\Gamma\left(\frac{1}{4}\right) \Gamma\left(\frac{3}{4}\right)}{\Gamma(1)}$.
(c) $\frac{\widetilde{I}_{n, 0}^{2}}{\widetilde{I}_{0, n}^{2}} \leq \frac{1}{3}$

Demonstração. De fato,
(a) $\frac{\widetilde{I}_{1, n-1}^{2}}{\widetilde{I}_{0, n}^{2}}=\frac{\frac{1}{4} \Gamma\left(\frac{1}{4}\right) \Gamma\left(n-\frac{1}{4}\right)}{\Gamma(n+1)} \frac{\Gamma(n+1)}{\Gamma\left(\frac{1}{4}\right) \Gamma\left(n+\frac{3}{4}\right)}=\frac{1}{4} \frac{\Gamma\left(n-\frac{1}{4}\right)}{\left(n-\frac{1}{4}\right) \Gamma\left(n-\frac{1}{4}\right)}=\frac{1}{4\left(n-\frac{1}{4}\right)}$ 


$$
\begin{aligned}
& \text { Logo } \frac{n-1}{2} \frac{\widetilde{I}_{1, n-1}^{2}}{\widetilde{I}_{0, n}^{2}}=\frac{n-1}{n-\frac{1}{4}} \frac{1}{8}<\frac{1}{8} . \\
& \text { (b) } \frac{\mathcal{B}}{n}=\frac{3}{4 n} \frac{\Gamma\left(\frac{1}{4}\right) \Gamma\left(\frac{3}{4}\right)}{\Gamma(1)} \leq \frac{(4 n-1)(4 n-5)(4 n-9) \ldots 11.7}{4 n-4)(4 n-8(4 n-12) \ldots 8.4} \frac{3}{4 n} \frac{\Gamma\left(\frac{1}{4}\right) \Gamma\left(\frac{3}{4}\right)}{\Gamma(1)} \\
& =\frac{(4 n-1)(4 n-5)(4 n-9) \ldots 11.7 .3}{4^{n} n(n-1)(n-2)(n-3) \ldots 3.2 .1} \frac{\Gamma\left(\frac{1}{4}\right) \Gamma\left(\frac{3}{4}\right)}{\Gamma(1)} \\
& =\frac{\Gamma\left(\frac{1}{4}\right)\left(n-\frac{1}{4}\right)\left(n-\frac{5}{4}\right)\left(n-\frac{9}{4}\right) \ldots \frac{11}{4} \frac{7}{4} \frac{3}{4} \Gamma\left(\frac{3}{4}\right)}{\Gamma(n+1)}=\frac{\Gamma\left(\frac{1}{4}\right) \Gamma\left(n+\frac{3}{4}\right)}{\Gamma(n+1)}=\widetilde{I}_{0, n}^{2} \\
& \text { (c) } \frac{\widetilde{I}_{n, 0}^{2}}{\widetilde{I}_{0, n}^{2}}=\frac{\Gamma\left(n+\frac{1}{4}\right) \Gamma\left(\frac{3}{4}\right)}{\Gamma(n+1)} \frac{\Gamma(n+1)}{\Gamma\left(\frac{1}{4}\right) \Gamma\left(n+\frac{3}{4}\right)}=\frac{\left(n-\frac{3}{4}\right)\left(n-\frac{7}{4}\right)\left(n-\frac{11}{4}\right) \ldots \frac{5}{4} \cdot \frac{1}{4} \Gamma\left(\frac{1}{4}\right) \Gamma\left(\frac{3}{4}\right)}{\Gamma\left(\frac{1}{4}\right)\left(n-\frac{1}{4}\right)\left(n-\frac{5}{4}\right)\left(n-\frac{9}{4}\right) \ldots \frac{7}{4} \frac{3}{4} \Gamma\left(\frac{3}{4}\right)} \\
& =\frac{(4 n-3)(4 n-7)(4 n-11) \ldots 5.1}{(4 n-1)(4 n-5)(4 n-9) \ldots 7.3} \leq \frac{1}{3} \text {. }
\end{aligned}
$$

Fato A.0.37
(a) $\frac{\widetilde{I}_{n-j, j}^{1}}{\widetilde{I}_{1, n-1}^{1}} \leq 1$ para $1 \leq j \leq n-1$
(b) $\frac{\widetilde{I}_{0, n-1}^{3}}{\widetilde{I}_{0, n}^{1}} \leq 2$ para $n \geq 2$.
(c) $\frac{\widetilde{I}_{1, n-1}^{1}}{\widetilde{I}_{0, n}^{1}} \leq \frac{1}{4\left(n-\frac{3}{4}\right)}$ para $n \geq 1$.
(d) $\frac{\widetilde{I}_{1, n-2}^{3}}{\widetilde{I}_{0, n}^{1}}<\frac{1}{4\left(n-\frac{3}{4}\right)}$ para $n \geq 3$.
(e) $\frac{\widetilde{I}_{i, n-1-i}^{3}}{\widetilde{I}_{1, n-2}^{3}} \leq 1$ para $n \geq 3$.

Demonstração.

$$
\text { (a) } \begin{aligned}
\frac{\widetilde{I}_{n-j, j}^{1}}{\widetilde{I}_{1, n-1}^{1}} & =\frac{\Gamma\left(n-j+\frac{1}{4}\right) \Gamma\left(j+\frac{1}{4}\right)}{\Gamma\left(\frac{5}{4}\right) \Gamma\left(n-\frac{3}{4}\right)}=\frac{\overbrace{\left(n-j-\frac{3}{4}\right)\left(n-j-\frac{7}{4}\right) \ldots \frac{9}{4} \frac{5}{4}}^{(n-1)} \text { parcelas }}{\Gamma\left(\frac{5}{4}\right) \Gamma\left(j+\frac{1}{4}\right)} \\
& =\frac{(4 n-(4 j+3))(4 n-(4 j+7)) \ldots 9.5}{(4 n-7)(4 n-11) \ldots(4 j+5)(4 j+1)} \leq 1
\end{aligned}
$$


(b) $\frac{\widetilde{I}_{0, n-1}^{3}}{\widetilde{I}_{0, n}^{1}} \leq 2$ por $(b)$ do Fato A.0.35

(c) $\frac{\widetilde{I}_{1, n-1}^{1}}{\widetilde{I}_{0, n}^{1}}=\frac{\frac{1}{4} \Gamma\left(\frac{1}{4}\right) \Gamma\left(n-\frac{3}{4}\right)}{\Gamma\left(n+\frac{1}{2}\right)} \frac{\Gamma\left(n+\frac{1}{2}\right)}{\Gamma\left(\frac{1}{4}\right) \Gamma\left(n+\frac{1}{4}\right)}=\frac{\Gamma\left(n-\frac{3}{4}\right)}{4 \Gamma\left(n+\frac{1}{4}\right)}$

$$
=\frac{\Gamma\left(n-\frac{3}{4}\right)}{4\left(n-\frac{3}{4} \Gamma\left(n-\frac{3}{4}\right)\right.}=\frac{1}{4 n-3}=\frac{1}{4\left(n-\frac{3}{4}\right)}
$$

(d) $\frac{\widetilde{I}_{1, n-2}^{3}}{\widetilde{I}_{0, n}^{1}}<\frac{1}{4\left(n-\frac{3}{4}\right)}$ por $(d)$ do Fato A.0.35

(e) $\frac{\widetilde{I}_{i, n-1-i}^{3}}{\widetilde{I}_{1, n-2}^{3}}=\frac{\Gamma\left(i+\frac{3}{4}\right) \Gamma\left(n-1-i+\frac{3}{4}\right)}{\Gamma\left(\frac{7}{4}\right) \Gamma\left(n-\frac{5}{4}\right)}=\frac{\Gamma\left(i+\frac{3}{4}\right) \Gamma\left(n-i-\frac{1}{4}\right)}{\Gamma\left(\frac{7}{4}\right) \Gamma\left(n-\frac{5}{4}\right)}$

$$
\begin{aligned}
& =\frac{\left(i-\frac{1}{4}\right)\left(i-\frac{5}{4}\right) \cdots \frac{11}{4} \frac{7}{4} \Gamma\left(\frac{7}{4}\right) \Gamma\left(n-i-\frac{1}{4}\right)}{\Gamma\left(\frac{7}{4}\right)\left(n-\frac{9}{4}\right)\left(n-\frac{13}{4}\right) \cdots\left(n-i+2-\frac{5}{4}\right)\left(n-i+1-\frac{5}{4}\right) \Gamma\left(n-i+1-\frac{5}{4}\right)} \\
& =\frac{(4 i-1)(4 i-5) \cdots 11.7}{(4 n-9)(4 n-13) \cdots(4 n-4 i+3)(4 n-4 i-1)} \leq 1 \text {, pois } 0<i \leq n-2
\end{aligned}
$$

Fato A.0.38 Para $n \geq 1$, temos $(a) \frac{\widetilde{I}_{n, 0}^{2}}{\widetilde{I}_{0, n}^{2}} \leq \frac{1}{3}$, e (b) $\frac{\widetilde{I}_{1, n-1}^{2}}{\widetilde{I}_{0, n}^{2}}=\frac{1}{4\left(n-\frac{1}{4}\right)}$.

Se $0<j \leq n-1$ então $\left(\right.$ c) $\frac{\widetilde{I}_{n-j, j}^{2}}{\widetilde{I}_{1, n-1}^{2}}<1$.

Demonstração. De fato,

(a) $\frac{\widetilde{I}_{n, 0}^{2}}{\widetilde{I}_{0, n}^{2}}=\frac{\Gamma\left(n+\frac{1}{4}\right) \Gamma\left(\frac{3}{4}\right)}{\Gamma(n+1)} \frac{\Gamma(n+1)}{\Gamma\left(\frac{1}{4}\right) \Gamma\left(n+\frac{3}{4}\right)}=\frac{\left(n-\frac{3}{4}\right)\left(n-\frac{7}{4}\right)\left(n-\frac{11}{4}\right) \ldots \frac{5}{4} \cdot \frac{1}{4} \Gamma\left(\frac{1}{4}\right) \Gamma\left(\frac{3}{4}\right)}{\Gamma\left(\frac{1}{4}\right)\left(n-\frac{1}{4}\right)\left(n-\frac{5}{4}\right)\left(n-\frac{9}{4}\right) \ldots \frac{7}{4} \frac{3}{4} \Gamma\left(\frac{3}{4}\right)} \leq \frac{1}{3}$

(b) $\frac{\widetilde{I}_{1, n-1}^{2}}{\widetilde{I}_{0, n}^{2}}=\frac{\frac{1}{4} \Gamma\left(\frac{1}{4}\right) \Gamma\left(n-\frac{1}{4}\right)}{\Gamma(n+1)} \frac{\Gamma(n+1)}{\Gamma\left(\frac{1}{4}\right) \Gamma\left(n+\frac{3}{4}\right)}=\frac{1}{4} \frac{\Gamma\left(n-\frac{1}{4}\right)}{\left(n-\frac{1}{4}\right) \Gamma\left(n-\frac{1}{4}\right)}=\frac{1}{4\left(n-\frac{1}{4}\right)}$

(c) $\frac{\widetilde{I}_{n-j, j}^{2}}{\widetilde{I}_{1, n-1}^{2}}=\frac{\left(n-j-\frac{3}{4}\right)\left(n-j-\frac{7}{4}\right) \cdots \frac{5}{4} \frac{1}{4} \Gamma\left(\frac{1}{4}\right) \Gamma\left(j+\frac{3}{4}\right)}{\frac{1}{4} \Gamma\left(\frac{1}{4}\right)\left(n-\frac{5}{4}\right)\left(n-\frac{9}{4}\right) \ldots\left(j+\frac{7}{4}\right)\left(j+\frac{3}{4}\right) \Gamma\left(j+\frac{3}{4}\right)}$

$$
=\frac{(4 n-(4 j+3))(4 n-(4 j+7)) \ldots 9.5}{(4 n-5)(4 n-9) \ldots(4 j+7)(4 j+3)}<1 \text {, }
$$

desde que $0<j \leq n-1$. 


\section{Referências Bibliográficas}

[Bar95] Robert G. Bartle. The elements of integration and Lebesgue measure. John Wiley \& Sons Inc., New York, 1995. Containing a corrected reprint of the 1966 original [The elements of integration, Wiley, New York; MR 34 \#293], A Wiley-Interscience Publication.

[CGM00] Anna Cima, Armengol Gasull, and Francesc Mañosas. Period function for a class of Hamiltonian systems. J. Differential Equations, 168(1):180-199, 2000. Special issue in celebration of Jack K. Hale's 70th birthday, Part 1 (Atlanta, GA/Lisbon, 1998).

[Kel76] Joseph B. Keller. Inverse problems. Amer. Math. Monthly, 83(2):107-118, 1976.

[Kre88] Erwin Kreyszig. Advanced engineering mathematics. John Wiley \& Sons Inc., New York, sixth edition, 1988.

[Lim76] Elon Lages Lima. Curso de análise. Vol. 1. Instituto de Matemática Pura e Aplicada, Rio de Janeiro, 1976.

[Lim81] Elon Lages Lima. Curso de análise. Vol. 2. Instituto de Matemática Pura e Aplicada, Rio de Janeiro, 1981.

[Rud76] Walter Rudin. Principles of mathematical analysis. McGraw-Hill Book Co., New York, third edition, 1976. International Series in Pure and Applied Mathematics.

[Rud99] Walter Rudin. Reelle und komplexe Analysis. R. Oldenbourg Verlag, Munich, 1999. Translated from the third English (1987) edition by Uwe Krieg. 
[Spi74] Murray R Spiegel. Manual de Fórmulas e Tabelas Matemáticas. E.U.A. McGraw-Hill, Inc., São Paulo, 1974. Coleção Schaum, translated from the third English (1973) edition Mcgraw-Hill do Brasil Ltda. 